RESEARCH

SERIES

NUMBER 91

September 2019

\section{AN ANALYSIS OF THE EFFECTS ON IRISH HOSPITAL CARE OF THE SUPPLY OF CARE INSIDE AND OUTSIDE THE HOSPITAL}

BRENDAN WALSH, MAEV-ANN WREN, SAMANTHA SMITH, SEAN LYONS, JAMES EIGHAN AND EDGAR MORGENROTH

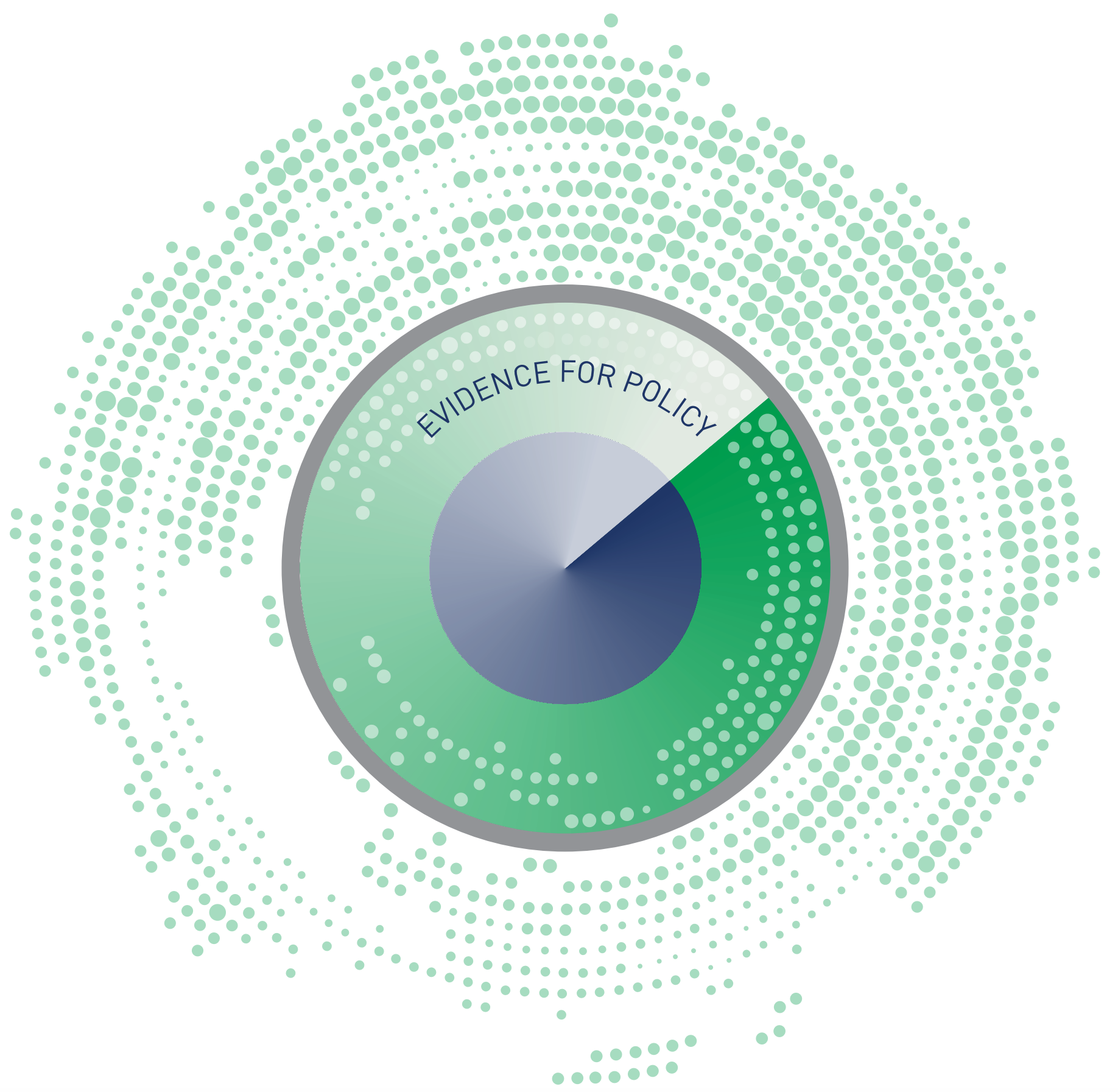





\section{AN ANALYSIS OF THE EFFECTS ON IRISH HOSPITAL CARE OF THE SUPPLY OF CARE INSIDE AND OUTSIDE THE HOSPITAL}

Brendan Walsh

Maev-Ann Wren

Samantha Smith

Seán Lyons

James Eighan

Edgar Morgenroth

September 2019

\section{RESEARCH SERIES}

\section{NUMBER 91}

Available to download from www.esri.ie

(C) The Economic and Social Research Institute

Whitaker Square, Sir John Rogerson's Quay, Dublin 2

ISBN: 978-0-7070-0500-3

DOI: https://doi.org/10.26504/rs91.pdf

Final report of the project entitled: 'An inter-sectoral analysis by geographic area of the need for and the supply and utilisation of health services in Ireland'

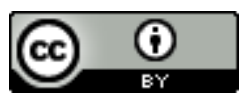

This Open Access work is licensed under a Creative Commons Attribution 4.0 International License, which permits unrestricted use, distribution, and reproduction in any medium, provided the original work is properly credited. 


\section{ABOUT THE ESRI}

The mission of the Economic and Social Research Institute is to advance evidencebased policymaking that supports economic sustainability and social progress in Ireland. ESRI researchers apply the highest standards of academic excellence to challenges facing policymakers, focusing on 12 areas of critical importance to 21 st Century Ireland.

The Institute was founded in 1960 by a group of senior civil servants led by Dr T.K. Whitaker, who identified the need for independent and in-depth research analysis to provide a robust evidence base for policymaking in Ireland.

Since then, the Institute has remained committed to independent research and its work is free of any expressed ideology or political position. The Institute publishes all research reaching the appropriate academic standard, irrespective of its findings or who funds the research.

The quality of its research output is guaranteed by a rigorous peer review process. ESRI researchers are experts in their fields and are committed to producing work that meets the highest academic standards and practices.

The work of the Institute is disseminated widely in books, journal articles and reports. ESRI publications are available to download, free of charge, from its website. Additionally, ESRI staff communicates research findings at regular conferences and seminars.

The ESRI is a company limited by guarantee, answerable to its members and governed by a Council, comprising 14 members who represent a cross-section of ESRI members from academia, civil services, state agencies, businesses and civil society. The Institute receives an annual grant-in-aid from the Department of Public Expenditure and Reform to support the scientific and public interest elements of the Institute's activities; the grant accounted for an average of 30 per cent of the Institute's income over the lifetime of the last Research Strategy. The remaining funding comes from research programmes supported by government departments and agencies, public bodies and competitive research programmes.

Further information is available at www.esri.ie. 


\section{THE AUTHORS}

Brendan Walsh is a Research Officer, Maev-Ann Wren is a Senior Research Officer, Samantha Smith is Research Associate and Seán Lyons is an Associate Research Professor at the Economic and Social Research Institute (ESRI). James Eighan was a Research Assistant at the ESRI. Edgar Morgenroth is a full Professor of Economics in DCU Business School, Dublin City University, Dublin. Brendan Walsh, Maev-Ann Wren and Seán Lyons have adjunct research positions at Trinity College Dublin.

\section{ACKNOWLEDGEMENTS}

The authors would like to thank the members of the Steering Group and collaborators on the project team for their expert advice and input. The authors would also like to thank people who provided additional analysis for the study.

The authors are very grateful to all those who facilitated the many requests for data for this study, in particular: Alan Cahill, Department of Health; Central Statistics Office; Justin Gleeson, All-Ireland Research Observatory (AIRO), Maynooth University; Dr Howard Johnson and the Health Intelligence Unit, Health Service Executive; Vincent Kennedy, Department of Health; Tom O'Regan, Health Information and Quality Authority; Dr Conor Teljeur and Department of Public Health and Primary Care, Trinity College Dublin; Anne Nolan and Paul Redmond, ESRI; Des Williams, National HR Directorate, Health Service Executive; Austin Warters, Michael Fitzgerald and Eithne McAuliffe, Services for Older People, Health Service Executive; and Bob Hennessy and Margaret Cahill, Health Information Quality Authority. 



\section{ABBREVIATIONS}

$\mathrm{ACHI}$

AHP

AMAU

AMI

BIU

$\mathrm{CHO}$

COPD

CSO

$\mathrm{DoH}$

DRG

EAPMC

ED

EU

FEMPI

GMS

GP

$\mathrm{HCP}$

HIPE

Hippocrates

HPO

HRB

HSE

ICD-10

IHI

IP

ISA

LHO

LOS

LSAS

LTRC

MAU
Australian Classification of Health Interventions

Allied health professionals

Acute medical assessment unit

Acute myocardial infarction (heart attack)

Business and Information Unit (in the HSE)

Community healthcare organisation

Chronic obstructive pulmonary disease

Central Statistics Office

Department of Health

Diagnosis-related group

Equitable Access to Primary Medical Care

Emergency department

European Union

Financial Emergency in the Public Interest

General Medical Services scheme

General practitioner

Home care package

Hospital In-Patient Enquiry

Healthcare in Ireland model of effects of Population Projections,

Patterns Of CaRe and Ageing Trends on Expenditure and Demand

for Services

Healthcare Pricing Office

Health Research Board

Health Service Executive

International Statistical Classification of Diseases and Related

Health Problems, 10th edition

Individual health identifier

Inpatient

Integrated Service Area

Local Health Office

Length of stay

Long-stay activity statistics

Long-term residential care

Medical assessment unit 
$\mathrm{NHI}$

NHS

NHSS

NTPF

OECD

PET

PHA

$\mathrm{PHI}$

PHN

RICO

SAT

SD

TILDA

UHI

UK

UQR

WTE
Nursing Homes Ireland

National Health Service (of the United Kingdom)

Nursing Home Support Scheme ('Fair Deal')

National Treatment Purchase Fund

Organisation for Economic Co-operation and Development

Patient experience time

Private Hospitals Association

Private health insurance

Public health nurse

Regional Integrated Care Organisation

Single assessment tool

Standard deviation

The Irish Longitudinal Study on Ageing

Universal health insurance

United Kingdom

Unconditional quantile regression

Whole-time equivalent 


\section{GLOSSARY}

Activities of daily

living (ADL)

Bed days

Capitation

Co-payment

Delayed discharge

Diagnosis-related

Group (DRG)

Disability

Elasticity

EU15

EU28

Fee-for-service

General Medical

Services Scheme

GP Visit Card

Health Information and Quality Authority (HIQA)

Health Service

Executive (HSE)

Hippocrates Model
An index that measures difficulties with personal tasks (e.g. eating or dressing), which is used as a proxy for severe disability.

Days in which hospital/nursing home beds are used.

A payment method where a healthcare provider receives a set amount for each enrolled person per time period, whether or not that person seeks care.

An out-of-pocket payment for care which is partially financed by the State or another source.

A patient who remains in hospital after a senior doctor (consultant or registrar grade) has documented in the medical chart that the patient can be discharged.

A system to classify hospital cases into a diagnosis-specific group.

This term covers impairments, activity limitations and participation restrictions where an impairment is a problem in body function or structure. An activity limitation is a difficulty encountered by an individual in executing a task or action, while a participation restriction is a problem experienced by an individual in involvement in life situations.

The percentage change in a dependent variable associated with the equivalent percentage change in an independent variable.

The 15 European Union Member States prior to 1 May 2004.

The current European Union Member States, since 1 July 2013.

A payment method where a separate payment is made to a healthcare provider for each medical service provided to a patient.

A scheme in which individuals who are eligible for a medical card receive mostly free access to public health services.

A card that allows the eligible recipient free GP visits.

An independent authority established in 2007 to monitor and promote quality and safety in Irish health and social care services.

The organisation that administers public health and social care services in Ireland.

The model developed by the ESRI to project future healthcare demand and expenditure. 
Home care package

Home help

Instrumental activities of daily

living (IADL)

Legacy funded

residents

Limited-stay beds

Local health office

Long-stay beds

Length of stay (LOS)

Mean

Medical assessment unit

Medical card

Morbidity

National Treatment

Purchase Fund

Nursing Home

Support (Fair Deal)

Scheme

Occupancy rate
A publicly-provided set of health and domestic services under the home care package scheme.

A service that provided domestic and personal care to individuals in their own home.

An index that measures difficulties with household tasks (e.g. cooking or shopping).

Residential long-term care residents who are funded though schemes that existed prior to the introduction of the NHSS in 2009.

Short-term residential care beds, including beds used for rehabilitation or convalescence after an illness/injury; palliative care for patients at a time 'when the medical expectation is no longer cure'; and respite, for 'the planned admission of dependent persons for short periods of time in order to assist carers in their task of caring'.

A HSE administrative geographic division (32 in total).

Residential care beds, including those for extended/continuing care for people who have been assessed as being in need of long-term care; psychiatry of old age, for specialised psychiatric services; and 'young chronic sick' for young people with a long-lasting illness that is usually irreversible and may be progressive.

The time, expressed in days, between admission to and discharge from hospital.

The arithmetic average of a group of numbers.

A facility whose primary function is the immediate and early specialist management of patients in a dedicated location for the quick assessment, diagnosis and initiation of appropriate treatment for these patients. It is also referred to as an acute medical assessment unit (AMAU) or an acute medical unit (AMU).

A card that allows the recipient free access to most public health services.

The state of being ill or having a disease.

A state body established in 2002 to reduce waiting lists in the public hospital sector.

A scheme through which the state funds or subsidises the care of residents in long-term care institutions based on an assessment.

The rate of available beds occupied or in use in a hospital or residential institution. 
Out-of-pocket

payments

Primary care team

Quantile

RICO

Standard deviation

Standardised variable

Tier 1 emergency department (ED)
Personal payments made by service users at the point of use.

A multidisciplinary group of health and social care professionals, including GPs and allied healthcare professionals, focused on the delivery of primary care.

Cut points in a distribution of a variable to divide the variable into equal size parts.

Proposed regional integrated care organisations, to replace $\mathrm{CHOs}$ and Hospital Groups.

A measure of the dispersion of a variable.

A variable rescaled to a mean equal to 0 and a standard deviation equal to 1.

ED providing emergency medical care 365 days a year, 24 hours a day. 



\section{FOREWORD}

This report was prepared by researchers at the Economic and Social Research Institute (ESRI) for the Health Research Board funded project, An inter-sectoral analysis by geographic area of the need for and the supply and utilisation of health services in Ireland. The report is the second ESRI Research Series Report published as part of this project. This report examines the effect of acute capacity, home care and long-term residential care on inpatient length of stay in public hospitals in Ireland.

The ESRI is responsible for the quality of this research, which has undergone national and international peer review prior to publication. This report was prepared by Dr Brendan Walsh, Dr Maev-Ann Wren, Dr Samantha Smith, Dr Seán Lyons, James Eighan and Prof Edgar Morgenroth; it reflects their expertise and views. The views expressed in this report are not necessarily those of other ESRI researchers, the Health Research Board, or organisations represented on the Steering Group.

September 2019 



\section{TABLE OF CONTENTS}

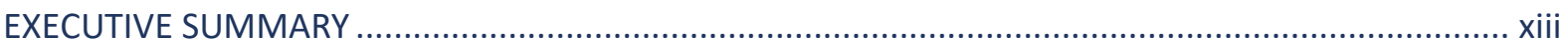

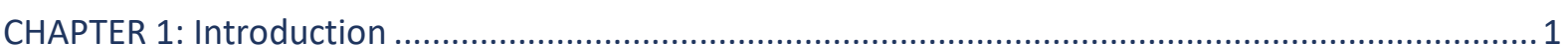

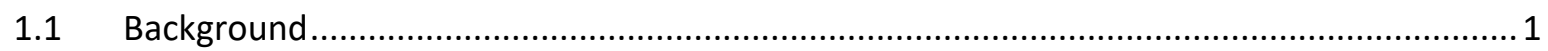

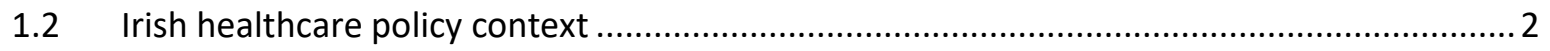

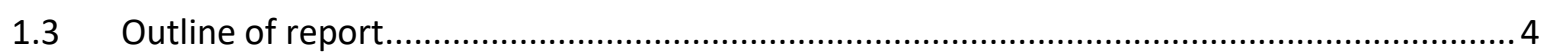

CHAPTER 2: Motivation for report and literature review ....................................................... 5

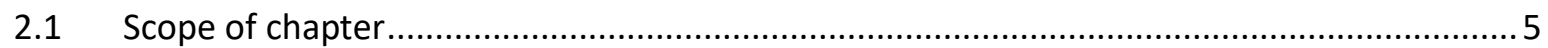

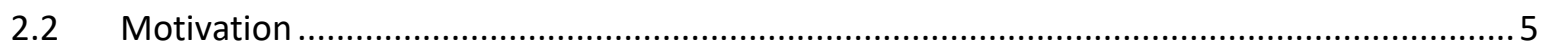

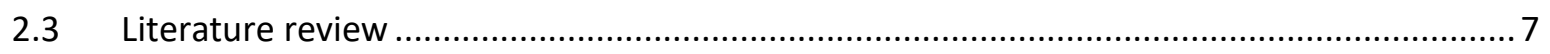

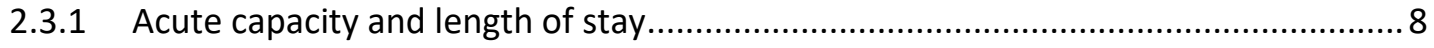

2.3.2 Long-term care capacity and inpatient length of stay........................................ 9

2.3.3 Irish evidence ............................................................................ 11

2.4 Contribution of this report to the literature .......................................................... 12

CHAPTER 3: Profile of acute care supply, home care and LTRC supply by geographic area ...............13

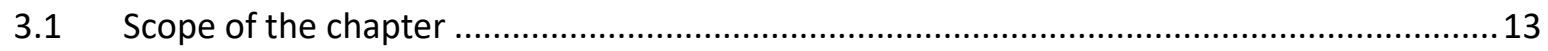

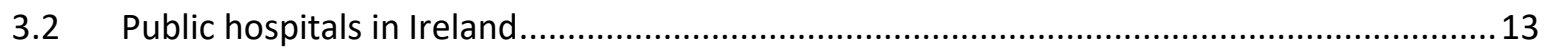

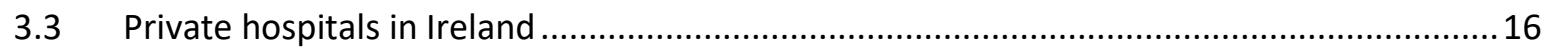

3.3.1 Private care in acute public hospitals .......................................................... 16

3.3.2 National Treatment Purchase Fund ............................................................... 18

3.4 Hospital In-Patient Enquiry (HIPE) dataset............................................................ 18

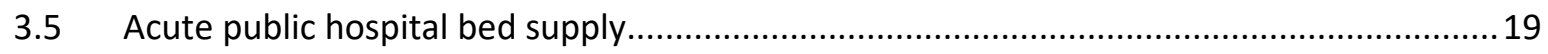

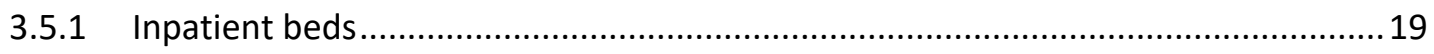

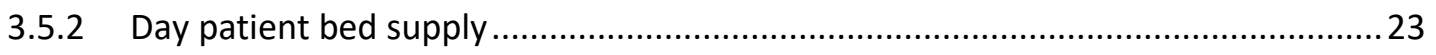

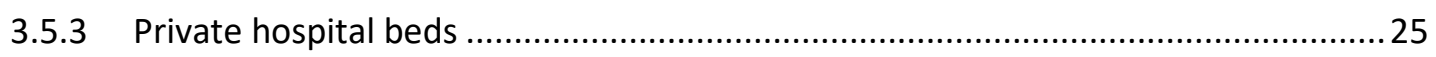

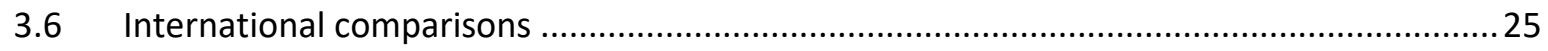

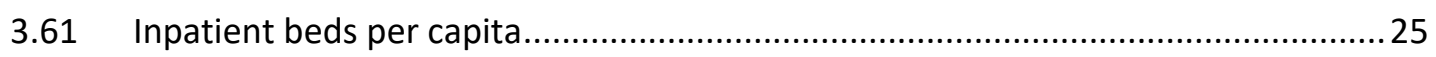

3.6.2 Inpatient bed occupancy rates ................................................................. 26

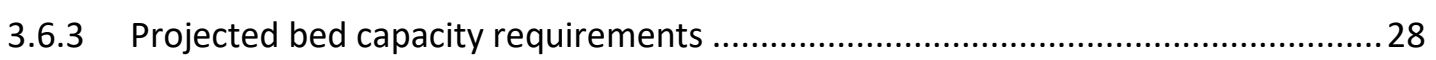

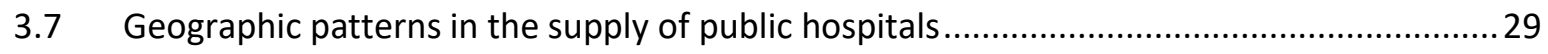

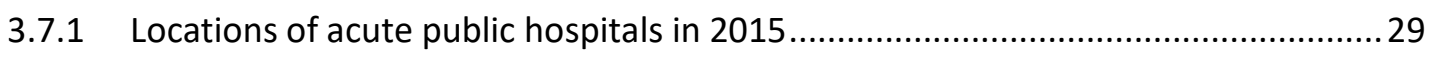

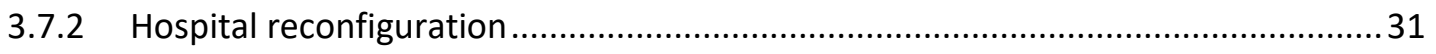

3.7.3 Acute public hospital catchment areas ........................................................ 34

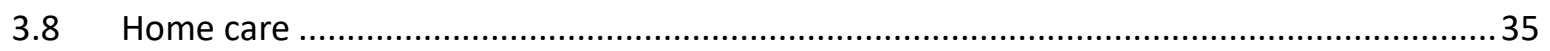

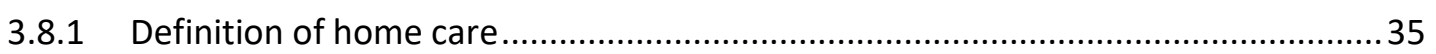

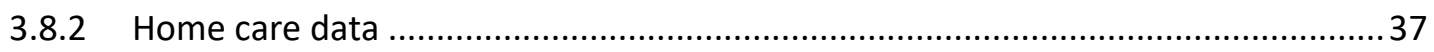

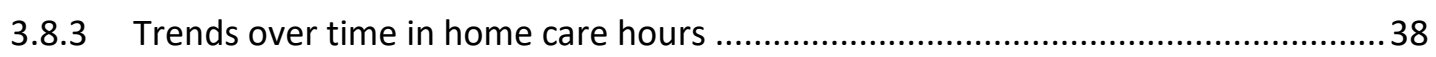

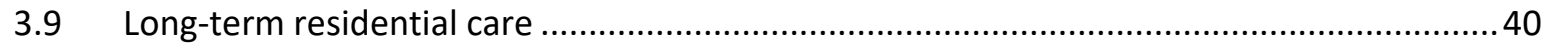

3.9.1 Definition of long-term residential care ....................................................... 40

3.9.2 Long-term residential bed data ......................................................... 41

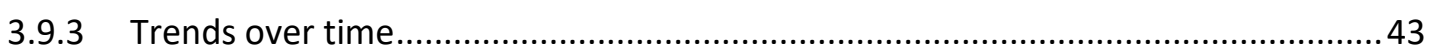


3.10 Home care and long-term residential care correlations ................................................. 45

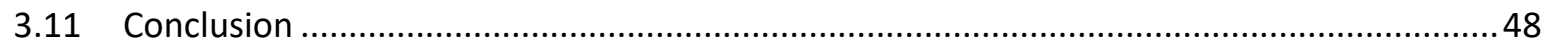

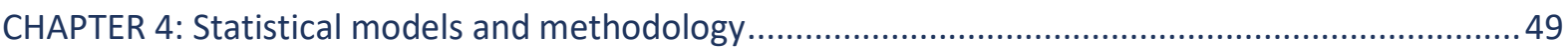

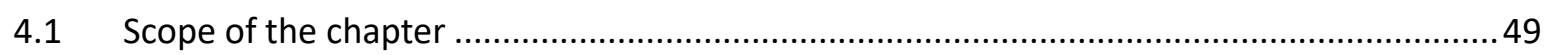

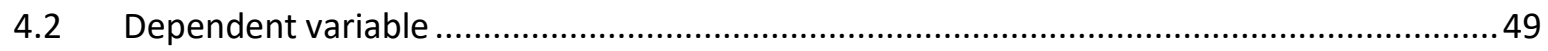

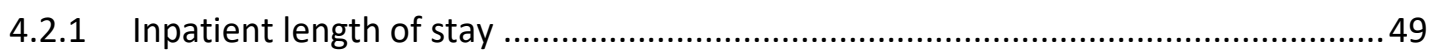

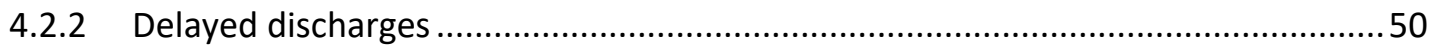

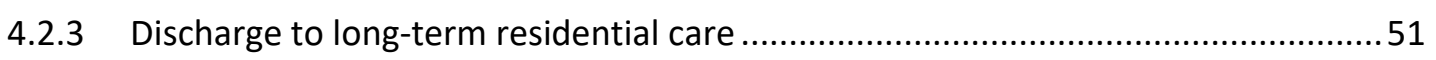

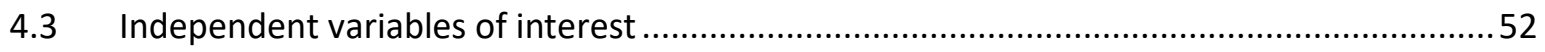

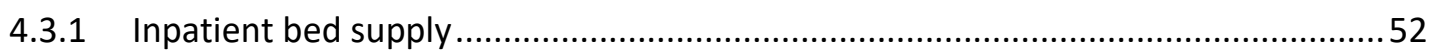

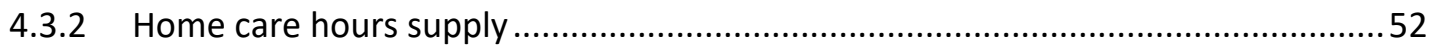

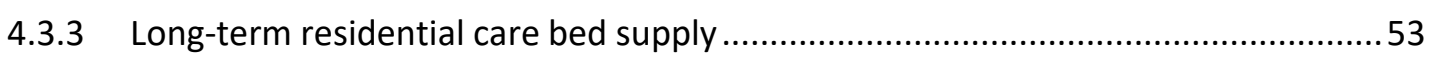

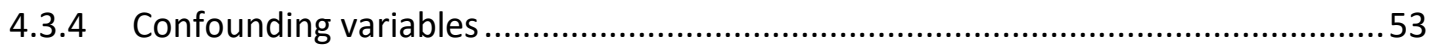

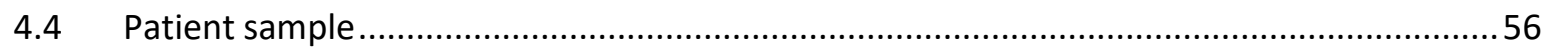

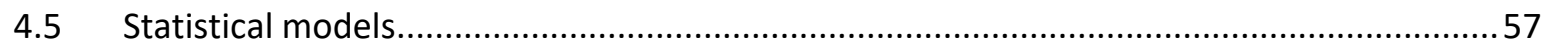

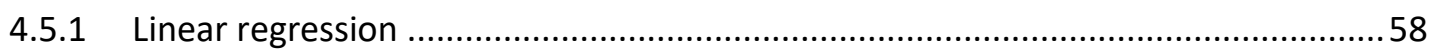

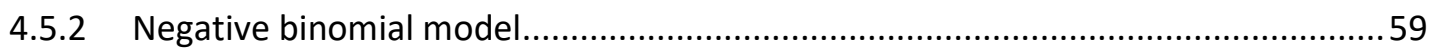

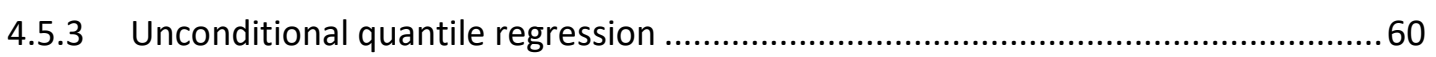

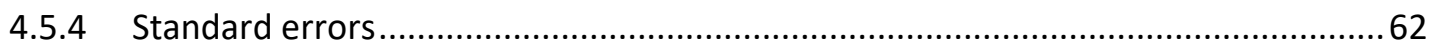

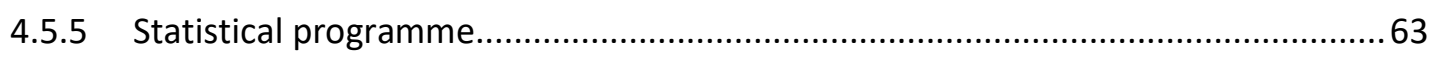

CHAPTER 5: Public hospital inpatient length of stay and inpatient bed supply..................................65

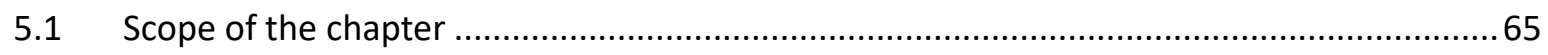

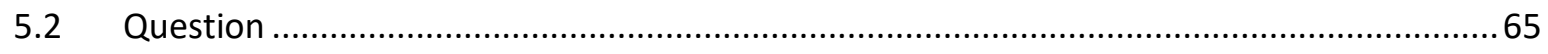

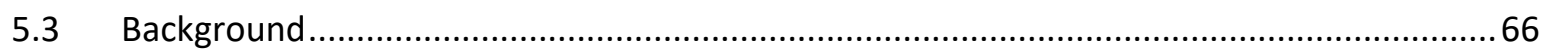

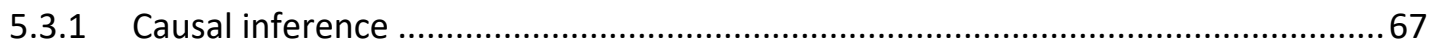

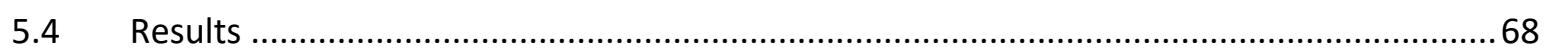

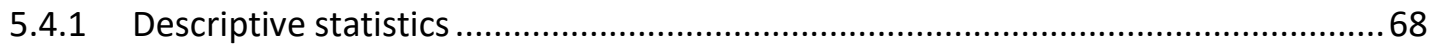

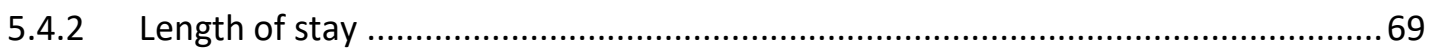

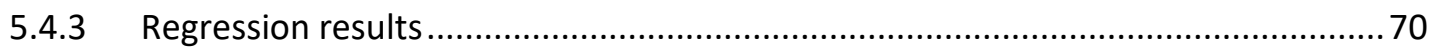

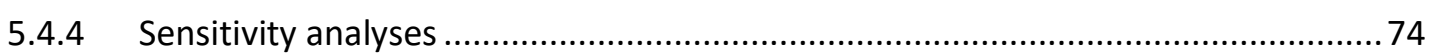

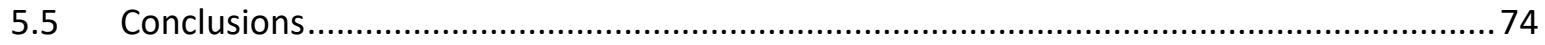

CHAPTER 6: Does formal home care reduce public hospital inpatient LOS and delayed discharges?.75

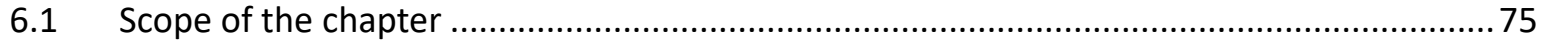

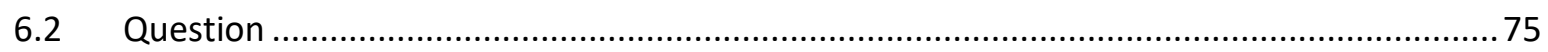

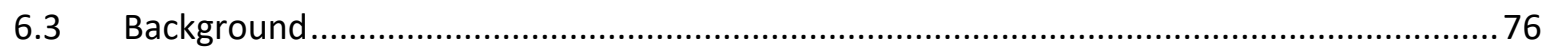

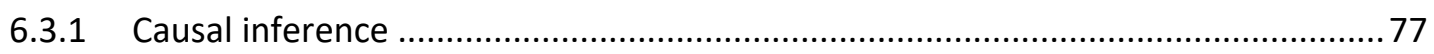

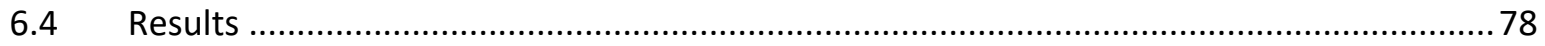

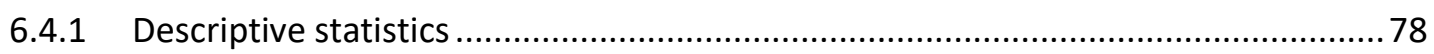

6.4.2 Length of stay and inpatient bed days .............................................................. 79

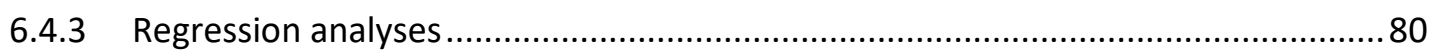

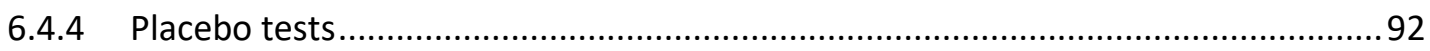

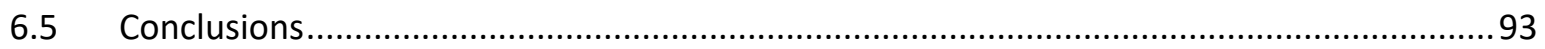


CHAPTER 7: Does LTRC reduce public hospital inpatient LOS and delayed discharges? .97

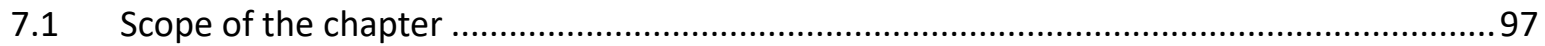

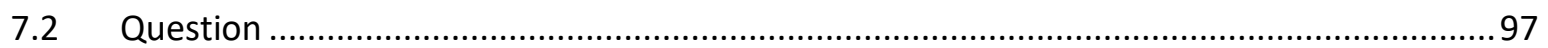

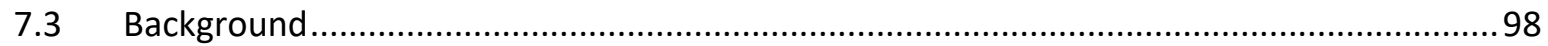

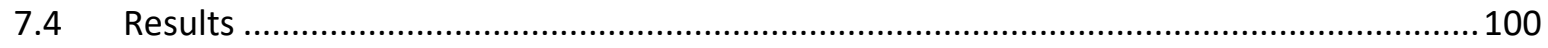

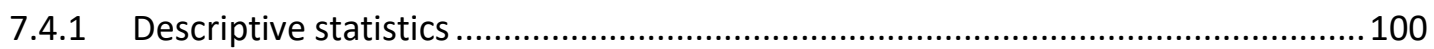

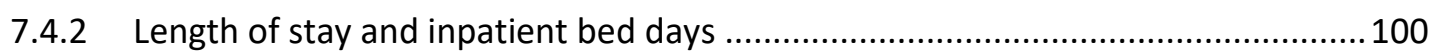

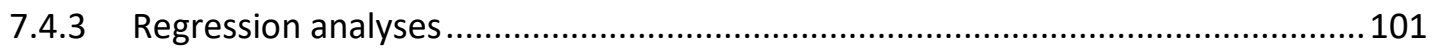

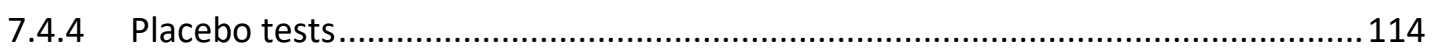

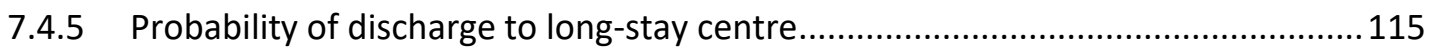

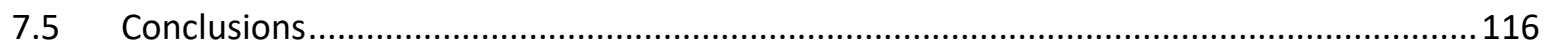

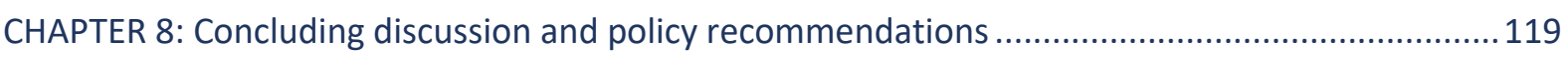

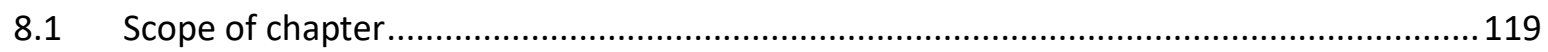

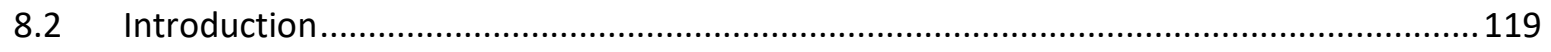

8.3 Geographic profile of healthcare needs and non-acute healthcare supply in Ireland (Smith

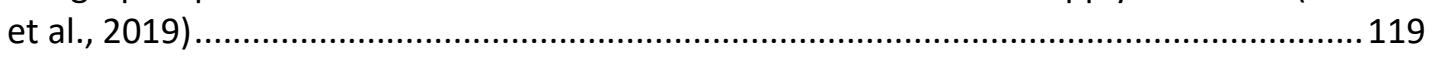

8.4 Analysis of the effects on Irish hospital care of the supply of care inside and outside the

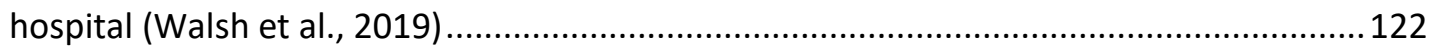

8.5 Policy recommendations for resource allocation............................................................. 128

8.5.1 Improved health system data ............................................................................

8.5.2 Better understanding of substitution and mechanisms to achieve integration of

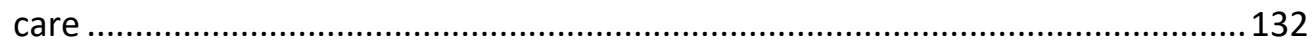

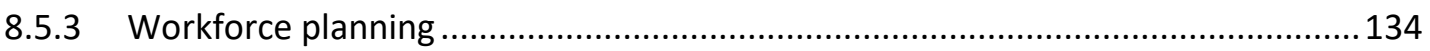

8.5.4 An allocation system designed to achieve equity of care supply relative to need

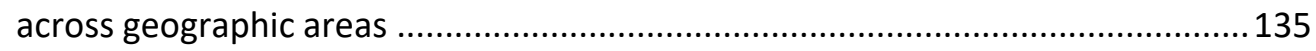

8.5.5 Regular review of resource allocation in line with regional demographic

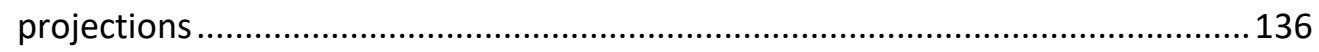

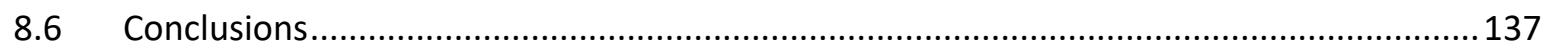

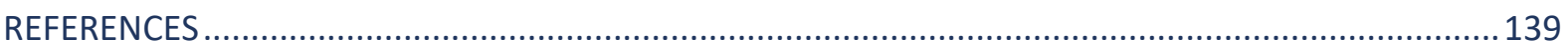

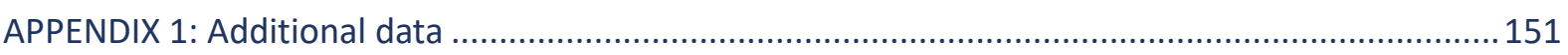

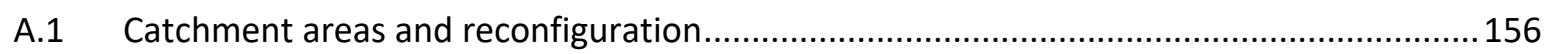





\section{EXECUTIVE SUMMARY}

\section{INTRODUCTION}

This report provides new evidence on key factors that affect patients' length of stay (LOS) in Irish public acute hospitals. Overall, the report finds that greater supply of home care and long-term residential care (LTRC) could reduce patients' LOS and thereby reduce delayed discharges, particularly for older people, in Irish hospitals.

This is the second report published from the Health Research Board-funded project, An inter-sectoral analysis by geographic area of the need for and the supply and utilisation of health services in Ireland. Findings in this report build on comprehensive evidence from Smith et al. (2019) on the geographic distribution of long-term care and community care in Ireland in 2014.

The overall objective of the project is to provide evidence to inform policymakers about the scope to move care from acute hospitals to other care settings in the community or LTRC (whether for longer stays or shorter-term rehabilitation or convalescence). This project is undertaken in the context of the Sláintecare (Houses of the Oireachtas Committee on the Future of Healthcare, 2017) reforms that seek to achieve greater integration in the Irish healthcare system and move delivery of care into the community where appropriate. The aim of this report is to contribute new evidence on key factors that affect patients' LOS and the extent of delayed discharges in public hospitals.

\section{DATA AND METHODS}

In this report, we examine public hospital inpatients from 2010 to 2015 using the Hospital In-Patient Enquiry (HIPE) database. We examine the impact of both 'push' factors - inpatient bed supply - and 'pull' factors - home care hours supply and LTRC bed supply. By comparing differences in acute and non-acute supply across regions and hospitals, and within regions and hospitals over time, and by controlling for patients' individual characteristics, we aim to identify the extent to which such factors influence patients' LOS.

Data on the number of acute inpatient beds for all acute public hospitals in Ireland between 2010 and 2015 were obtained from the Business and Information Unit (BIU) unit in the HSE. Data on publicly financed home care hours within the home help and home care package schemes across the 32 Local Health Offices for each month between 2012 and 2015 were provided by the HSE Social Care Division. Data on LTRC beds were estimated from a combination of Health Information and Quality Authority (HIQA) bed registry data, the Department of Health's Long-Stay 
Activity Statistics surveys, Nursing Home Ireland surveys, and Compliance Monitoring Inspection reports undertaken by HIQA. These data allow us to estimate the number of LTRC beds in each county for the years 2012 to 2015 inclusive.

We applied a number of modelling methods in this report to examine the impact of differences in the supply of home care and long-term care on patients' LOS, distinguishing between groups more or less likely to be classified as delayed discharges.

\section{MAIN FINDINGS}

The report finds that the LOS for older patients is shorter in counties with better supply of home care and LTRC services.

The magnitude of the link between greater home care supply and hospital LOS is greater for patients at older ages, patients with long LOS and patients with conditions which lead to particular reliance on home help services. Detailed findings for the effects of home care supply are as follows.

- For patients aged 65 years and over, a 10 per cent increase in per capita home care supply ( 1.5 million hours) was associated with at least a 1 per cent reduction in average LOS and a 1.3 per cent reduction in LOS for delayed discharges;

- An increase of 1.5 million hours in home care supply was associated with about 14,700 fewer inpatient bed days per annum, freeing up 40 inpatient beds daily. Among stroke and hip fracture patients, the effect was greater; a 10 per cent increase in per capita home care supply was associated with a 2.7 per cent and 1.6 per cent reduction in average LOS respectively.

- There is evidence that effects may be disproportionately stronger when there are large increments to the supply of home care. Analysis from Dublin North, which experienced large increases in home care supply during the period studied, finds that a 10 per cent increase in per capita home care supply was associated with a 2.7 per cent reduction in average LOS and a 6.9 per cent reduction in LOS for delayed discharges.

- This would equate to 40,000 fewer inpatient bed days per annum if applied nationally, freeing up 110 inpatient beds daily.

The magnitude of the relationship between greater LTRC supply and hospital LOS is greater for patients at older ages, patients with longer LOS and patients who are eventually discharged to LTRC care facilities. Detailed findings for the effects of LTRC supply are as follows.

- For patients aged 65 years and over, a 10 per cent increase in per capita LTRC bed supply was associated with a 1.3-2.2 per cent reduction in average LOS. 
This equates to about 19,000 fewer inpatient bed days per annum or freeing up 53 inpatient beds daily.

- A 10 per cent increase in per capita LTRC bed supply was associated with a 5.3 per cent reduction in average LOS for delayed discharges.

- For hip fracture patients and patients with Alzheimer's disease or dementia aged 85 years and over, a 10 per cent increase in per capita LTRC supply was associated with a 2.5 per cent and 5 per cent reduction in average LOS respectively.

- Larger substitution effects were estimated for patients ultimately discharged to an LTRC centre.

Separately, this report examines the association between inpatient bed capacity and LOS over the period 2010-2015. During the economic recession there was a 13 per cent reduction in inpatient bed supply, with a slight increase since 2013. As inpatient bed supply fell and then recovered over time, average LOS followed a similar pattern, while inpatient bed occupancy rates increased to the highest in the OECD at 95 per cent. This suggests that lower LOS may simply be a consequence of removing beds rather than an attempt to provide care more efficiently. Overall, our analyses suggest that as much as 40-60 per cent of the reduction in inpatient LOS between 2010 and 2012 may have been due to lower inpatient bed capacity, with subsequent increases in LOS due to once more increasing supply. Due to the lack of an individual health identifier (IHI) we cannot estimate readmissions from lower LOS or the impact these reductions had on patient outcomes and population health. 
TABLE ES.1 EFFECTS OF HOME CARE AND LTRC SUPPLY ON EMERGENCY INPATIENT DISCHARGES AGED 65+, IN RELATION TO LOS AND DELAYED DISCHARGES

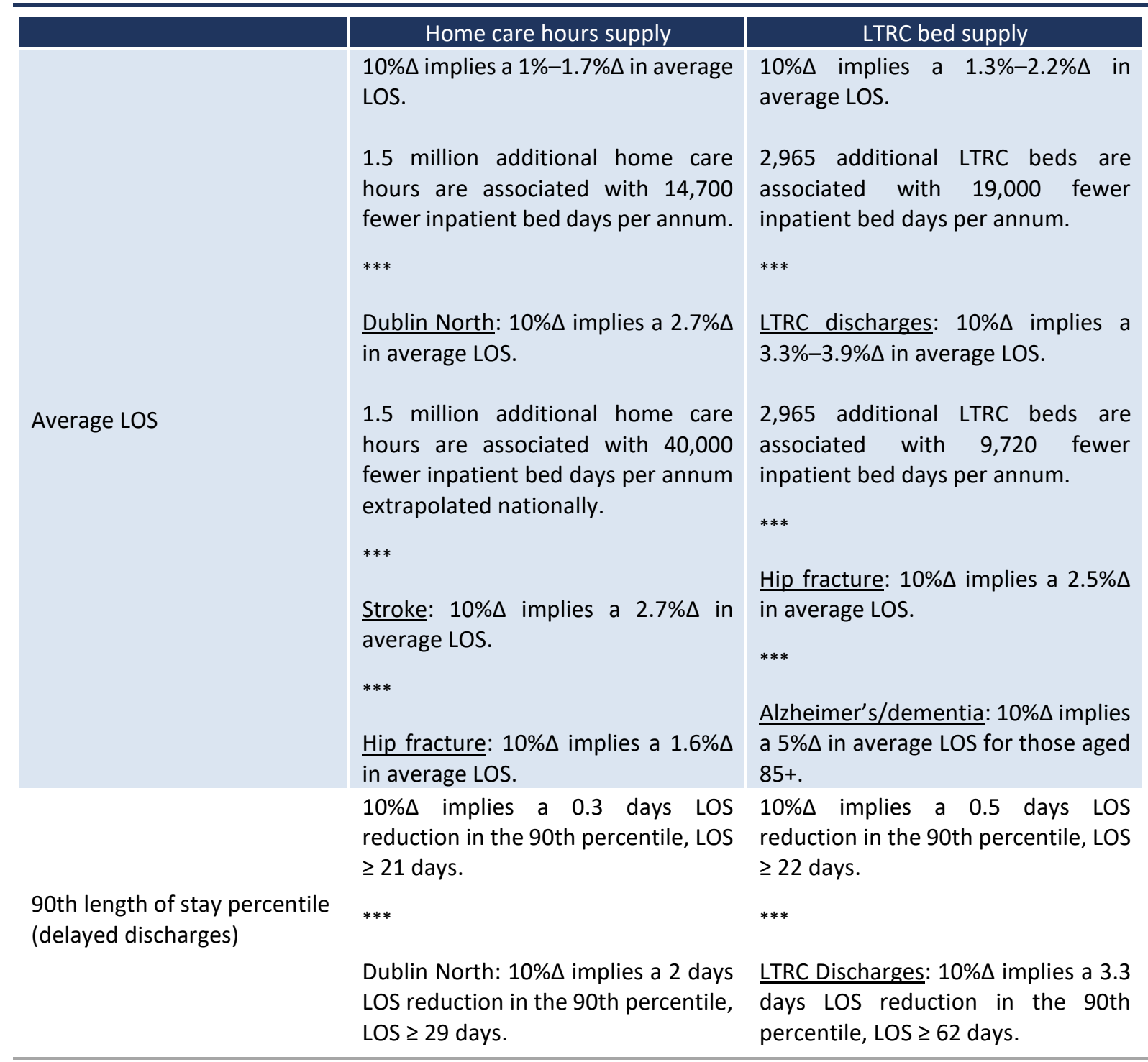

Notes: $\mathrm{SD}=$ standard deviation; $\Delta$ = change.

\section{CONCLUSIONS}

One of the 'strategic actions' of the Sláintecare Implementation Strategy involves the expansion of community-based care in Ireland. Bringing care closer to home, where appropriate and feasible, will require increases in workforces providing primary care, community care and long-term care services, as well as increased investment in LTRC beds. This report contributes to the evidence available on how increasing the supply of community-based care will affect demand for acute care in Ireland. The results show that better home care and LTRC provision are associated with shorter hospital LOS, especially among those groups whose care is most amenable to long-term care services, such as patients with stroke or hip fractures, and delayed discharges. Those geographical areas that received the largest increases in home care supply saw the largest reduction in hospital LOS. 
Overall, the findings of this project add significantly to the evidence base on health and social care in Ireland. In Smith et al. (2019), we found that there is substantial variation in the supply of non-acute services across regions, which does not reflect relative need. The east of the country fared particularly poorly. Having selected for more detailed examination the cases of home care and LRTC, in this report we have found an association between greater supply of such services and reduced duration of stay in acute hospitals, which is stronger for some patient groups than others. These headline findings from the two reports of this project suggest that hospital performance in Ireland may be driven by factors outside the control of the hospitals as well as within-hospital factors. Consequently, there is a risk that the system of activity-based funding that is being rolled out across acute hospitals may penalise hospitals inappropriately and further add to challenges for areas, which already have relatively inadequate non-acute services.

Greater provision of non-acute services such as home care is essential as the population grows and ages, especially as Ireland starts from a point of relative under-capacity in this area. We show that improving long-term care service supply can help to reduce hospital use, but that this is not a panacea for the pressures on the acute hospital system where significant capacity investment is required. Failure to adequately increase long-term care provision will further exacerbate the pressures on an acute system currently struggling to cope.

Inequitable supply of non-acute care across Ireland has arisen in an apparently arbitrary, historical manner, and reflects the absence of any system of planning for population health need. Ireland is unusual in not having a transparent and consistent system of resource allocation related to analysis of need. With rapidly increasing demographic pressures on the healthcare system and planned large capacity increases, introducing such a resource allocation method is a priority to optimise care and achieve cost-effective healthcare expenditure. Factors required to facilitate this transition to better resource allocation include: improved data collection; an evidence-based approach to potential substitution between differing services such as home care and hospital care; systematic methods to integrate care across settings; planning for the development and expansion of the healthcare workforce; and regular updating of resource allocation in line with regional population projections. 



\section{LIST OF TABLES}

Table ES.1: Effects of home care and long-term residential care supply on emergency inpatients aged $65+$ length of stay and delayed discharges ..........................................................

Table 3.1: List of acute public hospitals by emergency department status, 2015 ........................ 15

Table 3.2: Proportion of emergency inpatient discharges in Tier $1 \mathrm{ED}$ and other public hospitals in

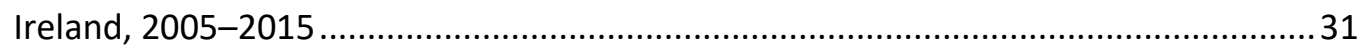

Table 3.3: Average annual number of publicly financed home care hours per person aged $65+$ by county, 2012-2015 ........................................................................................... 40

Table 3.4: Number of long-term residential care beds per 1,000 population aged $65+$ by county,

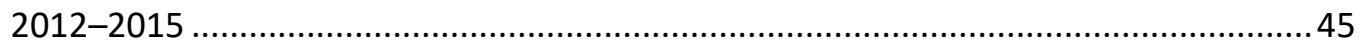

Table 5.1: Descriptive statistics of emergency inpatient discharges, 2010-2015.........................69

Table 5.2: Determinants of length of stay for emergency inpatient discharges (discharge-level model), 2010-2015 ............................................................................................... 71

Table 5.3: Determinants of length of stay for emergency discharges (hospital-level model), 2010-

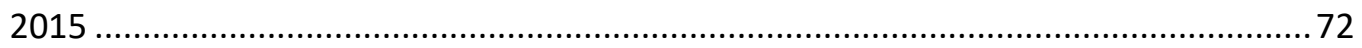

Table 6.1: Descriptive statistics of HIPE data for emergency inpatient discharges aged 65+, 2012-

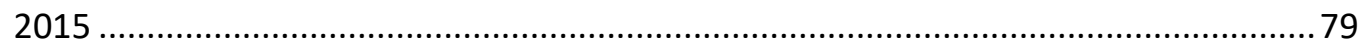

Table 6.2: Emergency inpatient discharges for patients aged 65+, 2012-2015 ........................... 81

Table 6.3: Emergency inpatient discharges for patients aged 65+, Dublin North, 2012-2015 ..... 86

Table 6.4: Emergency inpatient length of stay quantiles, Ireland and Dublin North, 2012 and 2015

Table 6.5: Emergency inpatient stroke discharges for patients aged 65+, 2012-2015

Table 6.6: Emergency inpatient hip fracture discharges for patients aged 65+, 2012-2015 ........ 91

Table 6.7: Determinants of length of stay for emergency inpatient discharges aged $<65$, Ireland and Dublin North, 2012-2015 …....................................................................... 93

Table 7.1: $\quad$ Descriptive statistics of HIPE data for emergency inpatient discharges aged 65+, 2012-

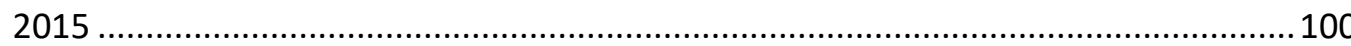

Table 7.2: $\quad$ Emergency inpatient discharges for patients aged 65+, 2012-2015 ........................102

Table 7.3: Emergency inpatient discharges discharged to long-stay facility for patients aged 65+,

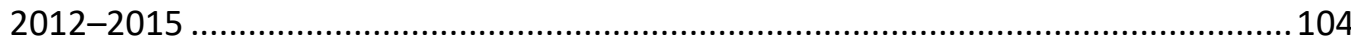

Table 7.4: Emergency inpatient stroke discharges for patients aged 65+, 2012-2015 .............. 109

Table 7.5: Emergency inpatient hip fracture discharges for patients aged 65+, 2012-2015 ......111

Table 7.6: Emergency inpatient Alzheimer's/dementia discharges for patients aged 65+, 2012-

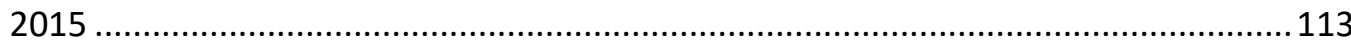

Table 7.7: Emergency inpatient discharges admitted from a long-stay facility for patients aged 65+, 2012-2015 .................................................................................................... 115

Table 7.8: Probability of discharge to a long-stay centre for emergency inpatient discharges: patients aged $65+, 2012-2015$

Table 8.1: Effects of acute and non-acute supply changes on emergency inpatient length of stay..

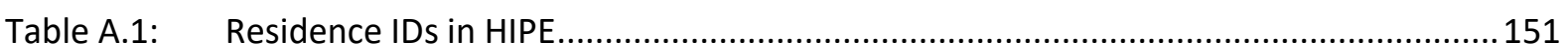

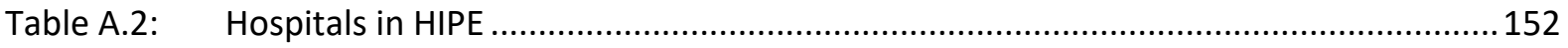

Table A.3: $\quad$ Average number of beds per LTRC centre by geographic area, Ireland, 2012-2015..157 


\section{LIST OF FIGURES}

Figure 3.1: Inpatient beds in public hospitals in Ireland, 1994-2015 ...........................................21

Figure 3.2: Inpatient beds per 1,000 population in public hospitals in Ireland, 1994-2015 ............ 22

Figure 3.3: Day patient beds in public hospitals in Ireland, 2003-2015 ......................................... 24

Figure 3.4: Day patient beds per 1,000 population in public hospitals in Ireland, 2003-2015 ......... 24

Figure 3.5: Acute inpatient bed capacity per 1,000 people across OECD countries, 2015* ............. 26

Figure 3.6: Inpatient bed occupancy rates across OECD countries, 2015*....................................28

Figure 3.7: Location of acute public hospitals with Tier 1 emergency department in Ireland ...........30

Figure 3.8: Drive time to a Tier 1 emergency department, 2005, 2010 and 2015 ............................33

Figure 3.9: Proportion of emergency inpatient discharges in each area of residence across hospitals

Figure 3.10: Average annual number of publicly financed home care hours per person aged $65+$ by county, 2012-2015.

Figure 3.11: Number of long-term residential care beds per 1,000 population aged $65+$ by county, 2012-2015.

Figure 3.12: Scatterplots of home care hours and long-term residential care beds supply by county, Ireland, 2012-2015

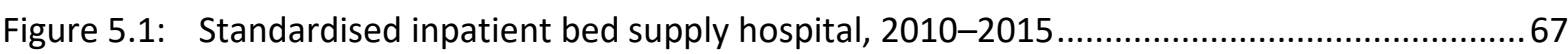

Figure 5.2: Average length of stay for emergency inpatient discharges, 2010-2015....................... 70

Figure 5.3: Emergency inpatient discharges, 2010-2015 …................................................... 73

Figure 6.1: Cumulative inpatient bed days by length of stay, emergency inpatient discharges aged 65+, 2012-2015

Figure 6.2: Determinants for length of stay for emergency inpatient discharges aged 65+ interaction plots, 2012-2015

Figure 6.3: Unconditional quantile regression for emergency inpatient discharges of patients aged $65+, 2012-2015$.

Figure 6.4: Unconditional quantile regression on emergency inpatient discharges, patients aged 65+ Dublin North, 2012-2015

Figure 7.1: Cumulative inpatient bed days by length of stay, emergency inpatient discharges aged $65+, 2012-2015$ 101

Figure 7.2: Determinants of length of stay for emergency inpatient discharges aged 65+, 20122015

Figure 7.3: Unconditional quantile regression, emergency inpatient discharges for patients aged 65+, 2012-2015.

Figure 7.4: Emergency inpatient discharges discharged to long-stay facility for patients aged 65+, 2012-2015 108

Figure 8.1 Summary of relative service supply indicators in Ireland, 2014

Figure A.1 Inpatient and day patient beds per 1,000 population aged $65+, 2010-2015$................ 154

Figure A.2 Proportion of discharges across most prevalent hospitals outside Dublin, 2015.........155

Figure A.3 Proportion of discharges across most prevalent hospitals outside Dublin, 2005, 2015156

Figure A.4 Average number of beds per centre by geographic area, Ireland, 2012-2015 ............157

Figure A.5 Comparison of model fit, Poisson and negative binomial.........................................158

Figure A.6 Unconditional quantile regression for emergency inpatient discharges age 18-44 years, Ireland and Dublin North. 


\section{CHAPTER 1}

\section{Introduction}

\subsection{BACKGROUND}

This is the final report of the project, An inter-sectoral analysis by geographic area of the need for and the supply and utilisation of health services in Ireland. The overall aim of the project is to inform public policy about the shift of care, where appropriate, from hospital settings to non-acute care settings (Smith et al., 2019). The analyses from both this report and the first report (Smith et al., 2019) were undertaken in the context of healthcare system reforms, significant capacity constraints and government policy prioritising a move towards greater provision of care in the community, as close to home as possible for patients.

Recent Irish health policy proposals recognise the necessity to move away from the current hospital-centric system and to achieve greater integration of health and social care services. Proposals such as the cross-party Sláintecare report (Houses of the Oireachtas Committee on the Future of Healthcare, 2017), the Department of Health's Health Service Capacity Review and the National Development Plan (2018-2027) provide the context underpinning the questions examined in this project. This project focuses on two areas where evidence is incomplete, and where evidence is vital to inform the future direction of the healthcare system and resource allocation decision making:

- whether or not there is adequate capacity outside of hospital to meet increased (or even existing) demands if more care were to be transferred to the non-acute sector; and

- the way in which acute and non-acute sectors interact and substitute in the system.

Smith et al. (2019) examine the first area, identified as vital for policy, and contribute to the evidence base on the supply of primary, community and longterm care services around the country. ${ }^{1}$ Smith et al. (2019) also provide evidence on the overall supply within the non-acute sector and geographic variation in supply, as well as estimates on the needs-adjusted supply according to healthcare need factors. Overall, Smith et al. (2019) find large inequalities in the per capita supply of the 10 services examined across counties in Ireland. These observed inequalities are not explained by differences in healthcare need factors, such as age, disability or morbidity. Consistent patterns in low supply emerged for some regions. The greater Dublin Commuter Belt counties (Kildare, Meath and Wicklow) 
and the South East (Kilkenny, Wexford and Waterford) had low relative supply for at least seven of the services examined. The findings from Smith et al. (2019) emphasise the requirement for a suitable resource allocation mechanism and the establishment of a data infrastructure to inform the efficient and equitable allocation of health and social care resources in Ireland.

The analyses undertaken in this report examines the second area vital for policymakers: understanding how health and social care sectors interact and substitute in Ireland. This report contributes new evidence on the interaction between hospital and long-term care in the Irish healthcare system. It examines substitution effects between acute inpatient length of stay (LOS) in public hospitals and two indicators of long-term care supply: home care and long-term residential care (LTRC).

This report also draws on key findings from this project and identifies and discusses other key barriers to make policy recommendations on the development of resource allocation for primary, community and long-term care services in Ireland.

\subsection{IRISH HEALTHCARE POLICY CONTEXT}

A number of policy proposals now provide frameworks for the future direction of the Irish health and social care system. The Government's health reform programme 2012-2015; the Health Service Capacity Review (PA Consulting, 2018), the Sláintecare report (Houses of the Oireachtas Committee on the Future of Healthcare, 2017), the Sláintecare Implementation Strategy (Government of Ireland, 2018b) and the Sláintecare Action Plan (Department of Health, 2019) all detail the need to shift away from the current 'hospital-centric' model of care and towards the development of a new integrated model of care whereby patients are treated at the lowest level of complexity and as close to home as possible. However, many of the points included in these proposals may be characterised as aspirational targets, at least in the short term. Proposals often lack the level of granular detail required to allow policymakers and local health planners to make decisions in the most efficient manner. Therefore, additional evidence is needed to help policymakers plan the details of the move between these models of care.

The Sláintecare Implementation Strategy outlines 10 'strategic actions' to be realised in the short term, in order to begin the process outlined in the original Sláintecare report. Two actions are key to the analysis in this report: one focuses on expanding community-based care to bring care closer to home; a second outlines the inclusion of the Health Service Capacity Review into the National Development Plan, and the need to develop and modernise the acute public hospital sector to address capacity inadequacies and to increase integration between the hospital sector and community-based care. It is acknowledged that in order to reduce pressures on the hospital sector, large increases in non-acute care 
supply and workforce are required. Two areas explicitly identified as requiring significant additional investment to reduce hospital capacity pressures are in-home care and LTRC.

In 2017, the first findings from the ESRI Hippocrates Model were published. These findings provided a comprehensive analyses of the demand for health and social care in 2015 in Ireland, and provided projected demand for care to 2030 (Wren et al., 2017). Large increases in demand were projected across all sectors between 2015 and 2030. However, the largest projected increases were estimated for home care and LTRC. Even allowing for some degree of healthy ageing among the older population in the future, the report projected demand increases for both services of between 40 and 54 per cent, not accounting for unmet demand currently in the system. ${ }^{2}$ This equates to an annual increase in demand for both services of between 2.7 and 3.6 per cent. Understanding the policy proposals in the context of these demand projections is vital. If care is substituted from hospitals, these projected demand increases will be even higher.

There is little published evidence, even internationally, on substitution between acute care and long-term care. Chapter 2 provides a detailed overview of the available evidence, but in brief, substitution effects are small, and research on the substitution in the NHS (Forder, 2009), Switzerland (Gonçalves and Weaver, 2017) and the US (Van Houtven and Norton, 2004) shows that increases in home care and LTRC (examined individually and collectively) has only a limited impact on reducing acute care utilisation. Therefore, understanding substitution effects in Ireland is important to the capacity plans included in the National Development Plan.

The Government has acknowledged that while a move away from the hospitalcentric system is required, current capacity within the acute sector is not sufficient. The National Development Plan has proposed an additional 2,600 hospital beds (day patient and inpatient) be added to the public hospital acute system by 2027 (Government of Ireland, 2018a). This planned expansion is based on an analysis of capacity need, which assumes substantial increases in non-acute care services where capacity will be required, in addition to the acute hospital expansion (PA Consulting, 2018). The projections in the acute sector are in the context of substantial changes to the acute public hospital sector since the mid-2000s. Chapter 3 outlines the large reconfiguration of the public hospital system that occurred post-2005, in which many hospitals saw their services, including emergency department (ED) services, reduced or removed. Public hospital inpatient bed supply reduced steeply, largely as a consequence of recessionimposed budget cuts, which resulted in changes in the geographic supply of acute 
services over time in Ireland, changes that have not been analysed within the literature. Therefore, providing information on the public hospital sector is also required, in order to provide insight into policy projections. We build on Smith et al. (2019), who provided details on the geographic variation in non-acute care supply, by undertaking similar analyses of the acute public hospital system in Ireland, and we also showed how acute bed capacity has a significant, but often overlooked, role in the use of hospital care.

\subsection{OUTLINE OF REPORT}

This report builds upon the international literature examining the interaction and substitution of hospital and long-term care services, by analysing substitution effects in Ireland. The analyses seek to examine:

- the distribution of acute services in Ireland in the context of reconfiguration of hospital services and changing patterns of acute bed supply;

- the impact bed supply changes have on inpatient LOS (a measure of utilisation);

- the changes in the geographic distribution of home care and LTRC services in Ireland over time; and

- substitution/interaction between inpatient LOS patients and supply of home care and LTRC for patients aged 65 years and over.

The report is outlined as follows.

- Chapter 2 sets out the motivation and background to the report, and introduces the literature on substitution in health and social care.

- Chapter 3 presents a profile of the hospital care, home care and LTRC sectors in Ireland. It details recent changes across each sector, geographic distribution in supply, as well as the data sources for each sector used within the analytical chapters.

- Chapter 4 discusses the data and methodologies used in the analytical chapters. In particular, it introduces the statistical methodologies and models, outlines the dependent and independent variables of interest and describes the types of patients included in the dataset.

- Chapter 5 examines the relationship between inpatient LOS and inpatient bed supply in public hospitals in Ireland between 2010 and 2015.

- Chapter 6 examines the relationship between inpatient LOS and per capita home care supply in Ireland between 2012 and 2015 for patients aged 65 years and over.

- Chapter 7 examines the relationship between inpatient LOS and per capita LTRC bed supply in Ireland between 2012 and 2015 for patients aged 65 years and over.

- Chapter 8 combines findings from Smith et al. (2019) and this present study to discuss potential resource allocation recommendations for acute and nonacute care in Ireland. 


\section{CHAPTER 2}

\section{Motivation for report and literature review}

\subsection{SCOPE OF CHAPTER}

This chapter outlines the motivation for this report. Section 2.2 details the background to the analysis in terms of planned changes to the Irish healthcare system. Section 2.3 reviews the existing academic literature on the effects of acute capacity, home care supply and long-term residential care (LTRC) supply on patient outcomes, hospital utilisation and inpatient length of stay (LOS). Section 2.4 outlines the contribution of this report to the literature.

\subsection{MOTIVATION}

Expanding care and service provision in the primary, community and long-term care sectors is a current priority for policymakers in Ireland (Department of Health, 2019; Government of Ireland, 2018b; Houses of the Oireachtas Committee on the Future of Healthcare, 2017). However, as discussed by Smith et al. (2019), there is only limited documented evidence on the patterns of supply of non-acute services across the counties in Ireland. Smith et al. (2019) built upon previous analyses of non-acute services and generated a more comprehensive picture of the geographic distribution of non-acute services in Ireland.

This expansion of care in the non-acute sector is envisaged to allow for care to be provided more appropriately outside of hospitals, and to remove much of the pressure on the overburdened hospital sector in Ireland. Expenditure on acute hospital care accounts for the largest proportion of healthcare spending in OECD countries (OECD, 2018). In 2016, hospital care accounted for 60 per cent of total healthcare expenditure in the $O E C D$, with inpatient services alone accounting for 30 per cent of total spend (OECD, 2018). In Ireland, 55 per cent of current healthcare expenditure was accounted for by the hospital sector in 2017 (CSO, 2019). ${ }^{3}$ Ireland is often seen as having a 'hospital-centric' service delivery model. This overburdening of the acute sector in Ireland has in part resulted in inpatient bed occupancy rates being the highest in OECD countries (OECD, 2018) and some of the longest waiting lists for elective care of developed countries (Siciliani et al., 2014). The overburdened public hospital sector is ill-equipped to meet the pressures on it, with one of the lowest bed-to-population ratios in the OECD (OECD, 2018). The Irish public hospital system has achieved a number of efficiency improvements in recent years, including a high proportion of procedures as day

3 Curative and rehabilitative care services provided by hospitals. 
cases (Wren et al., 2017) and one of the lower lengths of stay within the OECD (OECD, 2018); however, pressures on the acute sector remain.

Delivering care at the most appropriate level is a fundamental principle in the Sláintecare proposals for reform (Houses of the Oireachtas Committee on the Future of Healthcare, 2017). The Sláintecare report acknowledges the importance of developing non-acute care capacity in order to facilitate integrated care, as well as move away from a hospital-centric system. A key 'strategic action' of the Sláintecare Implementation Strategy focuses on expanding non-acute care services to provide adequate care supply closer to home (Department of Health, 2019). The Sláintecare report, the Department of Health's Capacity Review and the National Development Plan (2018-2027) set out a proposed future Irish health and social care system, and each report acknowledges that greater provision of acute services, such as inpatient beds, is required. This is largely due to the current inadequacy of acute service provision, as well as to projected growth and ageing of the population in coming decades (Wren et al., 2017). However, each plan's acute care projections are heavily dependent upon substantial expansion in nonacute care, especially in sectors that largely provide care for older people. These projections of acute care requirements are therefore dependent upon two very important elements: the ability of patients and health professionals to substitute care into the community; and the system's ability to expand workforce and capacity of non-acute services quickly. While the latter element is a vital component of healthcare reforms, in this report we focus on the former element - substitutability. We focus on two specific areas when examining substitutability between acute and non-acute care: home care and LTRC.

The report focuses on home care and LTRC for a number of reasons. First, these services are two of the largest components of non-acute care in Ireland more generally (Wren et al., 2017). In 2015, there were over 65,000 uses of public and private home care in Ireland. The State financed 10.46 million home help hours, with an additional estimated 3.86 million hours privately purchased. The State also provided 15,300 home care packages. Furthermore, there were an estimated 29,000 LTRC residents in 2015, with the majority (over 21,000) covered by the Nursing Home Support Scheme (NHSS, known as the 'Fair Deal' scheme). These residents used 10.6 million LTRC bed days - over twice as many inpatient bed days as in the public and private acute hospitals systems combined. While the ESRI report projected large increases in projected demand across the board, the largest projected increases were seen for these two services, and even accounting for healthy ageing in the older population in the future, demand for home care and LTRC care was projected to increase by 40-54 per cent between 2015 and 2030. In this context, any substitution of care from acute hospitals to home care and LTRC will likely result in even higher projected demand. 
Second, there is a dearth of granular data on healthcare utilisation in Ireland. There is a sufficient level of data to examine substitution effects in Ireland in acute public hospital care using the administrative Hospital In-Patient Enquiry (HIPE) data, as well as home care and LTRC, for which administrative data has been collected at regional level over a number of years.

Third, while there is limited national and international evidence on substitutability between acute and non-acute services, a small but revealing body of literature has developed around examining substitution effects between hospital care demand and expenditure and long-term care demand and expenditure. This literature is outlined in Section 2.3.

In this report, we also examine an oft-overlooked but key aspect: how acute capacity, changes in acute capacity and hospital reconfiguration can impact access to hospitals and hospital utilisation. The relationship between acute care and nonacute care is complicated and impacted by a range of demand-side and supply-side factors. In order to accurately examine the substitution effects of acute and nonacute care, where non-acute care may 'pull' patients out of hospitals into more appropriate care settings (such as an LTRC centre), we must also clearly understand that acute capacity constraints can 'push' patients out of hospital early, thereby reducing their LOS.

It is acknowledged that acute capacity at this moment in Ireland is insufficient; 2,600 additional hospital beds (day patient and inpatient) are now explicitly planned for within the National Development Plan (Government of Ireland, 2018a), with three elective care-only hospitals to be developed in Dublin, Cork and Galway by 2027. In this context, by examining the relationship between bed capacity and hospital use, we also shed light on how the provision of a defined number of acute beds that will be in a system at a given point in time may impact hospitalisations and inpatient LOS. In this report, we provide a detailed overview of the acute system, including the reconfiguration of services seen in recent years, and compare Ireland to international peers with regard to acute capacity. We also discuss the literature that examines how bed capacity impacts hospital use. Chapter 5 is dedicated to highlighting how inpatient bed capacity is also a key determinant of inpatient LOS.

\subsection{LITERATURE REVIEW}

Despite the fact that substitution of some aspects of care away from hospitals is a key policy recommendation of many health systems, there is a paucity of evidence on substitution between hospital care and non-acute care services. Few studies have determined how changes to acute capacity, such as removal of inpatient beds, can impact patient or population outcomes, as well as overall hospital use. In this section, we detail literature on how acute and non-acute care capacity may 
affect hospital care, in particular the expected effects on our dependent variable of interest: inpatient LOS.

\subsubsection{Acute capacity and length of stay}

In order to reduce the burden on acute hospitals, many health systems have conducted major reforms. Countries have removed care from acute public hospitals by greatly increasing non-acute capacity, replacing inpatient care with day patient care and providing incentives to reduce the length of inpatient stays such as through activity-based funding (ABF). There have been significant reductions in inpatient LOS over time across Europe and in developed countries generally. OECD statistics highlight that between 2000 and 2014, LOS reduced by almost 20 per cent on average in EU28 countries, from 9.9 days in 2000 to 8 days in 2014 (OECD, 2017b). Inpatient LOS reduced from 6.4 days to 5.6 days in Ireland over the same period and now ranks seventh lowest out of the 33 OECD countries examined (OECD, 2017b).

These reductions in inpatient LOS across health systems have been a result of a number of different factors. Within acute hospitals, greater use of more efficient surgery (Downing et al., 2009; Jayne et al., 2010; Laudicella et al., 2016), better discharge planning (Dedhia et al., 2009; Siegler et al., 2013), increasing use of palliative care planning (Brody et al., 2010), reductions in delayed discharges (McCoy et al., 2007; Rae et al., 2007) and more efficient payment mechanisms (Besstremyannaya, 2016; Borghans et al., 2008; Échevin and Fortin, 2014; Farrar et al., 2009) have been shown to reduce LOS. For specific patient populations, focused care units such as early supported discharge units and stroke units (Confalonieri et al., 2015; Keegan and Smith, 2013) and greater provision of non-acute follow-up and rehabilitation care have also been shown to allow patients to be discharged more quickly to potentially more appropriate care settings (Dahl et al., 2015; Gozalo et al., 2015; McCoy et al., 2007; Neiterman et al., 2015; Wren et al., 2014).

This long, but not exhaustive, list of factors shows that policymakers have many mechanisms with which to reduce inpatient LOS. However, the oft-overlooked factor, which may also drive lower LOS, relates to bed capacity available for patients to use. Lower LOS may be due to more efficient use of care but may also be indicative of a lack of staffing resources, reduced bed capacity, and/or higher bed occupancy rates. In this sense, whether lower LOS is actually valid as a performance measure can be questioned, as beyond a certain point lower LOS may result in negative consequences for the patient (Madsen et al., 2014).

Some studies have examined the impact of hospital bed supply, or a proxy of bed supply such as occupancy rates, on patient outcomes. A number of studies have examined the impact of bed supply changes in the Danish healthcare system. In the mid-2000s, Denmark embarked on a significant reorganisation of hospitals, 
centralising many services, and the number of acute hospitals reduced from 41 to 20 (Christiansen and Vrangbæk, 2017). A 16 per cent reduction in hospital beds occurred between 2007 and 2014, while inpatient LOS reduced from 3.9 days to 3.1 days (Christiansen and Vrangbæk, 2017). However, unlike the scenario in Ireland where bed cuts were accompanied by cuts to frontline staff numbers, in Denmark doctor and nurse whole-time equivalents (WTEs) actually increased by 19 per cent and 13 per cent respectively between 2007 and 2015 (Christiansen and Vrangbæk, 2017). Other research from Denmark on all admissions to Danish hospitals over a longer period of time has had contrasting results; in an examination of hospital care between 1995 and 2012, evidence of large inpatient bed shortages was found, with the subsequent high bed occupancy rates (greater than 90 per cent in many cases) associated with a 9 per cent increase in rates of inhospital mortality and thirty-day mortality as compared to lower occupancy rates (Madsen et al., 2014).

Studies on other health systems have also found a negative relationship between high bed occupancy and patient outcomes. In the NHS, a simulation study found that as occupancy rates exceeded 85 per cent, negative outcomes were seen, which were greatly exacerbated once the rate exceeded 90 per cent occupancy (Bagust et al., 1999). This was also found in a more recent study from the NHS, which showed that both higher occupancy and bed shortages in an NHS hospital were associated with higher patient mortality (Boden et al., 2016). Evidence from the US has found that higher occupancy and lower nursing WTEs were related to increased mortality (Schilling et al., 2010). A systematic review found lower bed capacity is associated with worse health outcomes, including increased mortality (Eriksson et al., 2017). It would be of substantial benefit to undertake similar analyses on the Irish system; however, due to data limitations a comprehensive study is not possible at present.

In the context of the relationships that exist between acute care supply, hospital use and patient outcomes, it is clear that prior to examining substitution effects in the Irish system, we first need to examine how inpatient bed capacity and inpatient LOS interact in Ireland. The extent to which lower LOS reduces acute capacity requirements, or vice versa, is a complex question, as outlined in many of the studies above. This issue is examined in Chapter 5 , through the use of granular data on inpatient utilisation and inpatient bed supply in Ireland.

\subsubsection{Long-term care capacity and inpatient length of stay}

Despite the fact that many health systems are moving care towards primary, community and long-term care settings, there is a relative paucity of evidence internationally on substitution between acute care and non-acute care. In general, with no quantitative evidence yet available for Ireland on the effects of improving 
capacity, or increasing the type and amount, of care provided in different settings, this makes the formulation of such policy difficult.

Some evidence exists on the interactions between acute and long-term care and social care in the NHS. A number of studies have found that increases in long-term care supply and expenditure reduced hospitalisations and inpatient LOS (Fernandez et al., 2018; Fernandez et al., 2013; Forder, 2009; Forder et al., 2018; Gaughan et al., 2017a; Gaughan et al., 2015; 2017b). However, substitution away from acute care to long-term care may not necessarily be cost saving. Forder (2009) found that increasing long-term care expenditure in England by $£ 1$ reduced acute hospital expenditure by $f 0.35$. In this sense, while increasing long-term care supply may not be cost saving, it may allow for patients to be cared for in more appropriate settings and free up valuable inpatient beds for elective care.

Where studies try to decompose substitutive long-term care effects into home care and LTRC, most of the substitutive effects are explained by LTRC supply, with only a weak, non-statistically significant relationship between home care supply and inpatient LOS found for those aged 65 years and over (Fernandez and Forder, 2008). Other work from the NHS found that both the number of delayed discharges and emergency inpatient readmissions are impacted by non-acute care provision, especially for older people (Fernandez and Forder, 2008). In this context, Gaughan et al. (2015) found that inpatient LOS and delayed discharges in particular respond to LTRC bed supply. The authors found that that a 10 per cent increase in LTRC beds reduces delayed discharges by between 6 per and 9 per cent.

While some studies from the US have argued that increased spending on home care has reduced hospital care expenditure (Lichtenberg, 2012), others have found little evidence of substitution away from post-acute facilities for a Medicare population with stroke, lower extremity joint replacement or hip fractures for patients with better access to home care (Huckfeldt et al., 2014). Home care has been found as a substitute for more skilled nursing home or intermediate care settings (Balentine et al., 2014). For some groups such as those at the end of life, home-based palliative care has also been shown to reduce hospital use and reduce costs (Chitnis et al., 2013).

Two studies from Switzerland (Gonçalves and Weaver, 2017) and Spain (Costa-Font et al., 2018) use geographic variation in home care supply (Switzerland) and home care allowances (Spain) across areas over time to try and determine the impact of home care on use of hospital services. As these studies rely on survey-based data, they examine the probability of hospitalisation over a period, number of hospitalisations over a period and LOS for the previous inpatient admission. Diverse results are found. Gonçalves and Weaver (2017) found that among those aged 65 years and older, no statistically significant reduction in LOS was observed 
in areas with more home care hours. However, the number of hospitalisations was shown to increase significantly as home care supply increased. While the authors do not test this result, it is possible that increased home care reduced LTRC, and the probability of requiring a hospitalisation may be lower for those in an LTRC centre as these patients can receive some form of acute care in an LTRC centre. Contrastingly, Costa-Font et al. (2018) showed that a policy that involves an increase in the amount of allowances available to informal carers and individuals to pay for formal care results in a reduction in inpatient LOS by up to 30 per cent, as well as reducing hospitalisation rates and hospital costs by 11 per cent (CostaFont et al., 2018). The allowances paid to informal carers appear to have the greatest effect on reducing hospital use, though the increased allowances provided to older individuals to pay for formal care were also found to reduce hospital use. The substitution effects found by Costa-Font et al. (2018) in terms of reduced LOS are much larger than found previously in the literature and may in part be a result of allowances rather than home care supply per se improving.

Many studies examining specific patient groups show that specialised treatment centre supply can greatly reduce acute care use as well as overall LOS for treatment. Evidence on patients receiving hip replacements in England found that patients in specialised public and private treatment centres had 18 per cent to 40 per cent shorter LOS compared to patients receiving care in acute public hospitals (Siciliani et al., 2013). For specific patient populations, focused care units such as early supported discharge units and stroke units (Confalonieri et al., 2015; Keegan and Smith, 2013) and greater provision of non-acute follow-up and rehabilitation care have also been shown to allow patients to be discharge more quickly to potentially more appropriate care settings (Dahl et al., 2015; Gozalo et al., 2015; McCoy et al., 2007; Neiterman et al., 2015).

\subsubsection{Irish evidence}

Evidence on substitution effects across healthcare sectors in Ireland is sparse. Much of the work examining differences in care between primary care and acute care uses changes in medical card or GP visit card access as the means to examine the impact. Recent studies found that expanding access to GPs for free, through the extension of free GP care to children aged under 6 years, increases GP visits ( $O$ 'Callaghan et al., 2018), but does not necessarily reduce emergency department (ED) attendance rates (Walsh et al., 2019). Other studies have shown improved GP access does not necessarily reduce hospitalisations overall (Ma and Nolan, 2016), or specifically for ambulatory care sensitive conditions - those conditions most appropriately treated in primary care amongst older people (Nolan, 2011). A clear negative relationship has also been found between primary care supply and hospitalisations for patients with certain chronic conditions such as chronic obstructive pulmonary disease (COPD) (Sexton and Bedford, 2016). 


\subsection{CONTRIBUTION OF THIS REPORT TO THE LITERATURE}

Overall, the literature on substitution effects between hospital and long-term care is ambiguous. One finding, however, that does appear to be consistent across studies is that even where a substitution effect between long-term care and acute care is found, the effects are small and LTRC supply dominates any substitution effect. However, results are context-specific; many results may not be as applicable an Irish setting due to the particular characteristics of the Irish health system. One objective of this report is to help fill this gap and inform future policy in Ireland.

This study builds upon the literature outlined above and models substitutive effects of home care and LTRC supply on inpatient LOS in public hospitals. In this context, we use differences in supply across areas and changes in supply over time within areas, controlling for a range of area-level, hospital-level and patient-level characteristics. We use this modelling strategy in an effort to isolate the causal impact that supply of care for older people has on reducing LOS. Using area-level data on long-term care supply is a common technique in the literature to examine substitution effects (Costa-Font et al., 2018; Fernandez and Forder, 2015; Forder, 2009; Forder et al., 2019; Gaughan et al., 2017a; Gonçalves and Weaver, 2017). 


\section{CHAPTER 3}

\section{Profile of acute care supply, home care and long-term residential care supply by geographic area}

\subsection{SCOPE OF THE CHAPTER}

This chapter presents a profile of acute hospital care, home care and long-term residential care (LTRC) in Ireland. It provides the institutional context and data sources for subsequent analytical chapters.

Sections 3.2 and 3.3 provide an overview of the acute hospital sector in Ireland, discussing both public hospitals (Section 3.2) and private hospitals (Section 3.3). In Section 3.4 we outline the Hospital In-Patient Enquiry (HIPE) dataset. Section 3.5 discusses acute inpatient bed supply and day patient bed supply in public hospitals; this section illustrates the changes of supply over time and across catchment areas, compares supply in Ireland to other jurisdictions internationally and discusses projected future bed requirements. Section 3.6 provides international comparisons. Section 3.7 details the supply of hospitals and hospital beds across regions, as well as the geographic catchment areas of public hospitals in 2015, considering how supply and distance to hospitals have changed over time as a result of reconfigurations of the system. Section 3.8 discusses supply of home care across counties over time. Section 3.9 discusses supply of LTRC across counties over time. Section 3.10 compares supply of home care and LTRC across counties. Section 3.11 concludes.

\subsection{PUBLIC HOSPITALS IN IRELAND}

The acute hospital sector in Ireland is a mixture of publicly-owned, voluntaryowned and privately-owned hospitals. Publicly-owned acute hospitals and acute hospitals owned by charity/voluntary organisations such as religious institutions are usually grouped together when the acute sector is discussed in Ireland, because both types are run on a not-for-profit basis, with the State providing almost all of the funding required (Tussing and Wren, 2006). In this context, we refer to all publicly-funded (public and voluntary) hospitals as 'public' hospitals in this report.

Historically, the location of public hospitals was distributed so that almost all 26 counties had an acute public hospital. In 2005, there were 59 public hospitals providing acute services as listed by the Department of Health (Tussing and Wren, 2006); in 2015, there were 53 acute public hospitals included by the Healthcare Pricing Office (HPO) as providing administrative data to HIPE (Healthcare Pricing Office, 2016a). ${ }^{4}$ While the number of hospitals has remained relatively constant,

$4 \quad$ The smaller number of hospitals in 2015 is mainly a result of closures and consolidation of some hospitals. 
the services provided by them have changed considerably; there now exists large differences in the services provided across hospitals. A subset of 29 public hospitals provides the vast majority of inpatient services (see Table 3.2). These 29 hospitals, across 28 sites, $^{5}$ each operate a Tier 1 emergency department (ED). These 29 EDs are open 24 hours a day, seven days a week, all year round and, generally, activity across them is used when calculating annual ED attendance rates in Ireland. ${ }^{6}$ Three of these hospitals located in Dublin provide care exclusively to children aged under 16 years of age (Walsh et al., 2019). In addition to the 29 adults' and children's hospitals, five maternity hospitals exclusively provide maternity care.

A further 11 medium-sized hospitals which have injury units (i.e. less comprehensive ED services) also provide emergency medicine (HSE, 2019). These units are often only open for selected hours and may transfer the more complex patients to a regional Tier 1 ED. Many of these hospitals previously had more comprehensive emergency medicine services, but saw a reconfiguration of their services in recent years (McHugh et al., 2019). Many still provide some emergency inpatient care, and also provide a large proportion of elective inpatient care. Other hospitals, no longer providing acute care, are not included in the list; many were previously district hospitals but have been reconfigured to provide short-term, rehabilitation or convalescent care.

Table 3.1 lists the largest public hospitals, including maternity hospitals, by their ED status in 2015. Table A.1 in Appendix 1 provides a more detailed list of hospitals and also provides information on changes to their ED status over time. This is not a comprehensive list of acute public hospitals (see HPO for more details), but the hospitals listed do provide the majority of acute care in the public system and are the main hospitals examined in later chapters of this report.

A children's hospital and an adults' hospital are co-located on the Tallaght University Hospital site. The HSE includes these 29 hospitals within their patient experience time (PET) administrative dataset used to collect information on EDs in Ireland. 


\begin{tabular}{|c|c|c|}
\hline Tier 1 Emergency departments & Injury units & Maternity hospitals \\
\hline Beaumont Hospital & Bantry Injury Unit & National Maternity Hospital at Holles St. \\
\hline Cavan General Hospital & Dundalk Injury Unit & Coombe Women and Infants University Hospital \\
\hline Connolly Hospital Blanchardstown & Ennis Injury Unit & Rotunda Hospital Dublin \\
\hline Cork University Hospital & Mallow Injury Unit & Midwestern Regional Maternity Hospital \\
\hline Kerry University Hospital & Mater Smithfield Rapid Injury Clinic & Cork University Maternity Hospital** \\
\hline Letterkenny University Hospital & Monaghan Injury Unit & \\
\hline Mater Misercordiae University Hospital & Nenagh Injury Unit & \\
\hline Mayo University Hospital & Roscommon Injury Unit & \\
\hline Mercy University Hospital - Cork & St. Columcille's Injury Unit, Loughlinstown & \\
\hline Midland Regional Hospital - Mullingar & St. John's Injury Unit, Limerick & \\
\hline Midland Regional Hospital - Portlaoise & The Mercy Injury Unit, Cork & \\
\hline \multicolumn{3}{|l|}{ Midland Regional Hospital - Tullamore } \\
\hline \multicolumn{3}{|l|}{ Naas General Hospital } \\
\hline \multicolumn{3}{|l|}{ Our Lady of Lourdes Hospital - Drogheda } \\
\hline \multicolumn{3}{|l|}{ Our Lady's Hospital - Navan } \\
\hline \multicolumn{3}{|l|}{ Portiuncula University Hospital - Ballinasloe } \\
\hline \multicolumn{3}{|l|}{ Sligo University Hospital } \\
\hline \multicolumn{3}{|l|}{ South Tipperary General Hospital } \\
\hline \multicolumn{3}{|l|}{ St. James's Hospital } \\
\hline \multicolumn{3}{|l|}{ St. Luke's General Hospital - Kilkenny } \\
\hline \multicolumn{3}{|l|}{ St. Vincent's University Hospital } \\
\hline \multicolumn{3}{|l|}{ Tallaght University Hospital - Adult ED \pm} \\
\hline \multicolumn{3}{|l|}{ University Hospital Galway } \\
\hline \multicolumn{3}{|l|}{ University Hospital Limerick } \\
\hline \multicolumn{3}{|l|}{ University Hospital Waterford } \\
\hline \multicolumn{3}{|l|}{ Wexford General Hospital } \\
\hline \multicolumn{3}{|l|}{ Children's University Hospital - Temple Street* } \\
\hline \multicolumn{3}{|l|}{ Our Lady's Children's Hospital - Crumlin* } \\
\hline Tallaght University Hospital - Paediatric ED*士 & & \\
\hline
\end{tabular}

Notes: $\quad *$ Children's hospitals. These provide emergency medicine to those aged under 16 years.

** Cork University Maternity Hospital is often included as Cork University Hospital, including within the HIPE dataset.

\pm Tallaght University Children's Hospital and Tallaght University Adults' Hospital are included together in HIPE and by the HSE when calculating acute bed capacity 
This report examines public hospitals, rather than private hospitals, for the following reasons:

- the public sector is most amenable to change from government policy;

- the data available for analysis on public hospital care is superior to that available on private hospital care;

- the majority of acute care in Ireland is provided in public hospitals (Wren et al., 2017); and

- the importance of substitution towards the non-acute setting is particularly pertinent for public hospitals.

\subsection{PRIVATE HOSPITALS IN IRELAND}

While a large proportion of private care in Ireland is provided in public hospitals, private hospitals still represent a significant component of the acute hospital sector. In 2015, there were 18 for-profit hospitals in Ireland that were members of the Private Hospital Association (PHA) (Private Hospitals Association, 2017). All PHA members are accredited by internationally recognised accreditation bodies such as the Joint Commission International or the Mental Health Commission (Private Hospitals Association, 2017). Private hospitals in Ireland mainly provide non-emergency care, elective inpatient care, day patient care and outpatient care. Private hospitals provide some limited emergency care, either with an ED or a less comprehensive medical assessment unit (MAU) operating in approximately half of its members (Private Hospitals Association, 2016). Attendance to EDs in private clinics is much lower than in public hospital EDs, with the largest chain of these private ED clinics (Vhi Swiftcare) having 84,000 attendances in 2016 (Vhi, 2017), compared to 1.14 million ED attendances in public hospital EDs in 2015 (Wren et al., 2017).

Patients in private hospitals must pay the full cost of their care and these hospitals cater mainly for individuals covered by private health insurance. Private hospitals are reimbursed by insurers based upon agreed rates for diagnostic and medical procedures (McLoughlin, 2014) and coverage of particular private hospitals differs across insurance policies. The role of the private hospital system in providing care has increased considerably over time; in 2015, private hospitals provided an estimated 31 per cent of all day patient admissions and 15 per cent of inpatient bed days in the acute hospital sector (Wren et al., 2017). The inpatient bed days provided are primarily accounted for by elective inpatients, with emergency inpatient care predominantly provided in public hospitals, even in the case of private patients.

\subsubsection{Private care in acute public hospitals}

The mixed public/private healthcare system in Ireland presents some unique complications when examining care provision that are not encountered in many other health systems. Public hospital care is available to all, either through 
relatively low charges, or free of charge for patients with a medical card. However, public hospitals also admit private patients, who pay private fees to hospital consultants and private overnight charges to hospitals (typically covered by insurance but potentially out of pocket) (Brick et al., 2010). A primary motivation for purchase of private health insurance is to avoid long waits for public hospital care (Harmon and Nolan, 2001; Kapur, 2019; O'Regan, 2014). Within public hospitals in Ireland, patients can be treated privately, may use a private or semiprivate bed, or be treated by a consultant who treats both public and private patients in the same hospital. The majority of emergency inpatient care also occurs in public hospitals, regardless of whether the recipient of care is a public or private patient.

In public hospitals, up to 20 per cent of inpatient beds are designated as 'private' or 'semi-private'. Private patients are charged for the use of these beds, with a private bed being charged more than a semi-private bed (Department of Health, 2017c). From 2014, private patients are also charged for use of any inpatient bed (regardless of whether or not it is designated private or semi-private) once they state that they are a private patient. ${ }^{7}$ Evidence has previously found that private inpatients use more inpatient bed days than are potentially available to them (O'Reilly and Wiley, 2010). In 2015, 19 per cent of all public hospital inpatients were privately financed and over 50 per cent of total private inpatient (elective and emergency) bed days across public and private hospitals were recorded in public hospitals (Keegan et al., 2018b). The Sláintecare report estimated that in 2015, $€ 650$ million was spent on private care in public hospitals; it recommended that private practice should be removed from public hospitals (Houses of the Oireachtas Committee on the Future of Healthcare, 2017). However, this figure does not necessarily represent capacity that could be freed up for public care by moving private cases out of the public system. A recent examination of activity in public and private hospitals in Ireland suggests that the private hospital system appears to primarily specialise in the delivery of elective care. It is therefore unclear whether the majority of private inpatients in public hospitals who are emergency inpatients could access the care they may require in private hospitals (Keegan et al., 2018b).

The distinction between public and private inpatients is often based upon whether a patient is treated as a private patient by their medical consultant, who receives a fee for their care. Medical consultants working in public hospitals can provide care to private patients in public hospitals as well as in private hospitals, depending upon the contract type agreed with the HSE. The Comptroller and Auditor General's Report on the accounts of the public services 2017 finds that 82 per cent of medical consultants working in public hospitals have contracts that allow them to provide care onsite (in a public hospital) to private patients for 20 per cent of 
their time, with the majority of these able to provide private outpatient and/or inpatient care off-site (in private hospitals) (Comptroller and Auditor General, 2018). It is unknown what proportion of medical consultants in Ireland work only in private care settings and hospitals.

\subsubsection{National Treatment Purchase Fund}

Some care in private hospitals is also purchased for public patients by the National Treatment Purchase Fund (NTPF). The NTPF is an independent statutory organisation established in 2004. The NTPF has a number of functions including negotiating pricing arrangements with LTRC centres under the NHSS and collecting waiting list information for individuals awaiting treatment in public hospitals. Under the latter remit, the NTPF arranges for the provision of elective inpatient and outpatient treatment for public patients in private hospitals to alleviate waiting lists in the public system. The NTPF does not collect information on the number treated or removed from waiting lists; rather, it collects the number of patients waiting for care monthly, by hospital, speciality and time waiting. Separate waiting lists are collected for outpatient and inpatient care. Therefore, it is difficult to estimate the number of public patients treated in private hospitals under the NTPF schemes. ${ }^{8}$

\subsection{HOSPITAL IN-PATIENT ENQUIRY (HIPE) DATASET}

The HIPE dataset is the main source of information on activity in the public hospital system in Ireland. (Private hospitals to not report activity to HIPE.) In this report, we use the HIPE dataset to examine hospital utilisation within public hospitals in Ireland over time. HIPE is a routinely collected administrative dataset that is sourced from the HPO. HIPE information is available for the years 2005-2015 inclusive. The HIPE scheme is a health information system that collects and collates clinical and administrative data on discharges from - and deaths in - all large public hospitals. As there is no unique individual health identifier (IHI) in Ireland, individual patients across multiple discharges or instances of use of other health services cannot be followed. For this reason, each HIPE record is collected at the discharge level, rather than at the patient level. A HIPE record represents one single episode of care, which may be either a day patient episode or an inpatient episode. In the case of inpatient care, while HIPE records whether or not the inpatient was admitted through an ED, no other information on the ED attendance itself is recorded. The HPO estimated that virtually all (99.9 per cent) of day and inpatient discharges in HIPE were coded and returned in 2015 (Healthcare Pricing Office, 2016a). 
A range of patient demographic information is recorded within the HIPE record. First, patient's area of residence and hospital of discharge are recorded. This information is used in this report to examine hospital catchment areas and to characterise the supply of acute and non-acute services. Within HIPE, patients' home addresses are used to construct a categorical variable divided into 49 Irish areas of residence (Resid). This Resid variable allows us to examine differences in inpatient length of stay (LOS) across areas in Ireland in this report. Additionally, those of 'no fixed abode' and the country of international patients are also recorded. The list of areas of residence is included in Table A.1. There are over 50 hospitals included in HIPE from 2005 to 2015. Table A.2 highlights these hospitals and details the years each hospital's records were collected in HIPE.

Other demographic variables collected by HIPE are: age; sex; medical card status; marital status; private health insurance (up to 2011); public/private status; ${ }^{9}$ and whether the discharge was a maternity, day patient, elective inpatient or emergency inpatient discharge. Other information recorded are: date of admission and of discharge; source of admission; disease classification (ICD-10-AM classifications); health procedure (ACHI classification); and diagnosis-related group (DRG). For all discharges, information is recorded on up to 20 diagnosis codes (one principal and up to 19 additional) and, where applicable, 20 procedure codes (one principal and up to 19 additional). Importantly for this report, LOS for each discharge is also recorded. Within HIPE, LOS refers to the time, expressed in days, between admission to and discharge from hospital (HIPE, 2009).

\subsection{ACUTE PUBLIC HOSPITAL BED SUPPLY}

In this section, we discuss the data on acute hospital bed supply in Irish hospitals in recent years, largely focusing on bed supply in acute public hospitals as the data available on private hospitals are much more limited. An acute hospital bed may fall into a number of categories, defined by the type of treatment provided and whether the patients stayed overnight. Commonly, acute beds are categorised as day patient, inpatient, critical care or palliative care beds. However, many specialised hospitals will include some types of interim care or rehabilitation beds. In this report we concentrate on the most common bed categories: day patient and inpatient.

\subsubsection{Inpatient beds}

An inpatient is a person who enters an acute hospital to receive curative, medical care and stays at least one night in hospital. The OECD provides a clear definition 
on what they consider a (curative) inpatient bed. ${ }^{10}$ Across the OECD, approximately 30 per cent of all healthcare expenditure is spent on inpatient care (OECD, 2018).

Inpatient bed supply in Ireland has been amongst the lowest in the OECD since at least the turn of the century (Department of Health and Children, 2002). While some other countries reduced the number of beds during the recent economic recession, Ireland cut hospital beds faster than most other countries in the OECD (OECD, 2017a).

Figure 3.1 illustrates the total number of available inpatient beds in public hospitals in Ireland between 1994 and 2015, using a combination of data from the Department of Health (pre-2008) and the HSE. ${ }^{11}$ Total available inpatient bed supply remained largely stable from the early 1990s until 2007. However, at the onset of the recent economic recession, there was a marked drop in available public inpatient beds, with a large number of beds and wards closed (not staffed) across hospitals. Between 2008 and 2012, available inpatient beds fell by over 13 per cent. A stabilisation in inpatient bed availability occurred post-2012 as public healthcare expenditure increased and the severest economic consequences of the recession subsided.

The drop in inpatient bed numbers post-2008 was largely a result of healthcare expenditure cuts brought about by the economic recession. However, this reduction also followed the publication of an acute bed capacity report commissioned by the HSE (PA Consulting, 2007). This report argued that efficiency improvements, greater integration of the health service and meeting international standards such as on LOS would result in a lower number of inpatient hospitals beds ( $<9,000$ beds) required by 2020 . Subsequent bed capacity analyses in 2018 found a need to reverse these cutbacks in light of high occupancy rates, unmet need for care and projected population growth (discussed further below) (PA Consulting, 2018).

10 The OECD definition for a curative (acute) care bed is: 'Beds accommodating patients where the principal clinical intent is to do one or more of the following: manage labour (obstetrics), cure non-mental illness or provide definitive treatment of injury, perform surgery, relieve symptoms of non-mental illness or injury (excluding palliative care), reduce severity of non-mental illness or injury, protect against exacerbation and/or complication of non-mental illness and/or injury which could threaten life or normal functions, perform diagnostic or therapeutic procedures' (OECD, 2012).

11 For Department of Health data, see https://health.gov.ie/publications-research/statistics/statistics-by-topic/publichospital-bed-numbers/. 


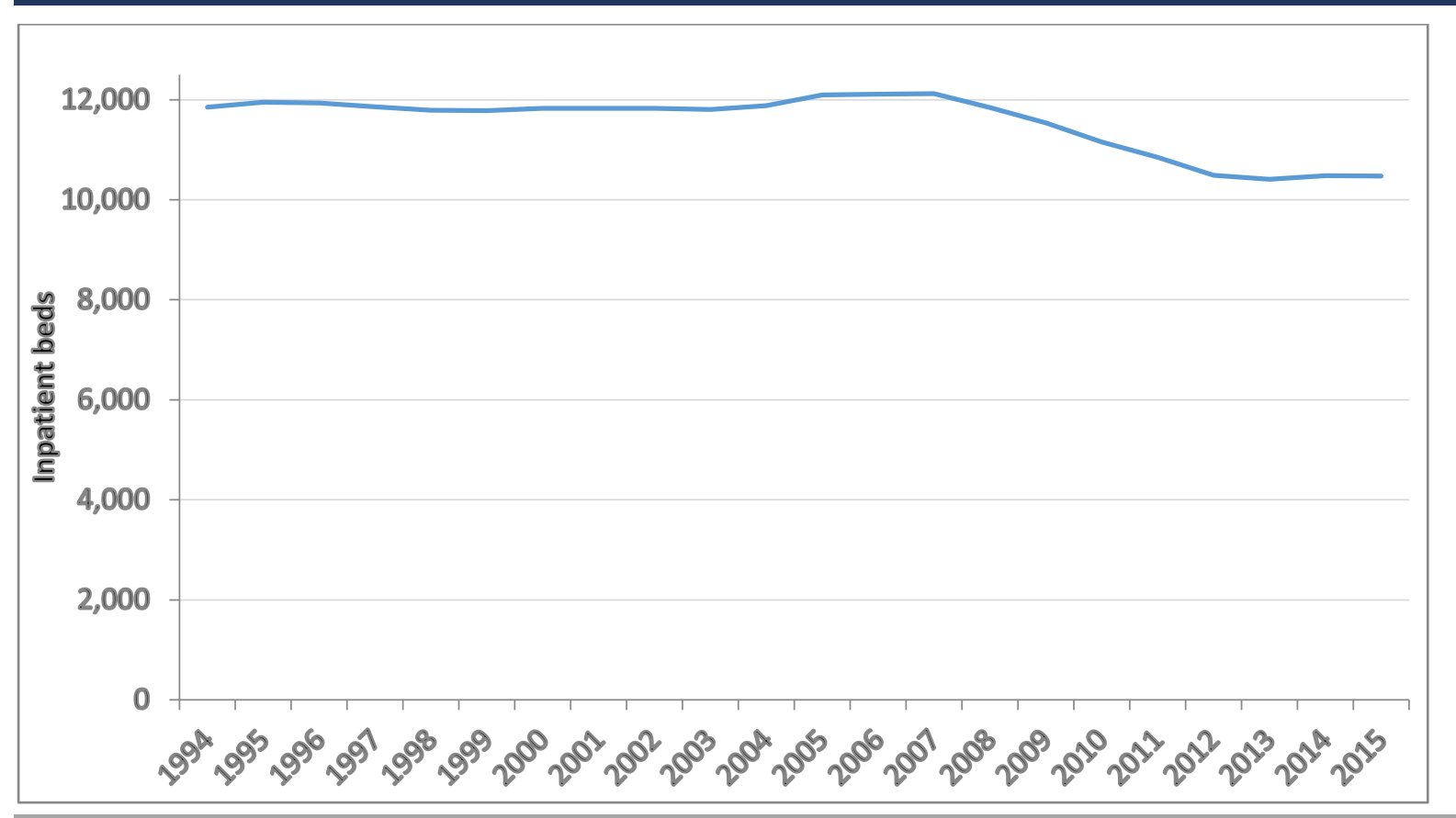

Source: Department of Health, BIU HSE.

While Figure 3.1 illustrates the trend in total numbers of beds, it understates the extent of changes in supply per capita. The reductions in inpatient bed capacity observed in recent years also occurred in the context of an increasing population, with the size of the population increasing by 30 per cent in the preceding two decades (Wren et al., 2017). Figure 3.2 illustrates the total number of available inpatient beds per capita (per 1,000 population) in public hospitals in Ireland between 1994 and 2015. The reduction in per capita supply showed a broadly linear trend over time, reducing from 3.31 beds per 1,000 in 1994 to 2.23 beds per 1,000 in 2015, which is equivalent to a 33 per cent reduction in inpatient beds per 1,000 people over this time period. 
FIGURE 3.2 INPATIENT BEDS PER 1,000 POPULATION IN PUBLIC HOSPITALS IN IRELAND, 1994-2015

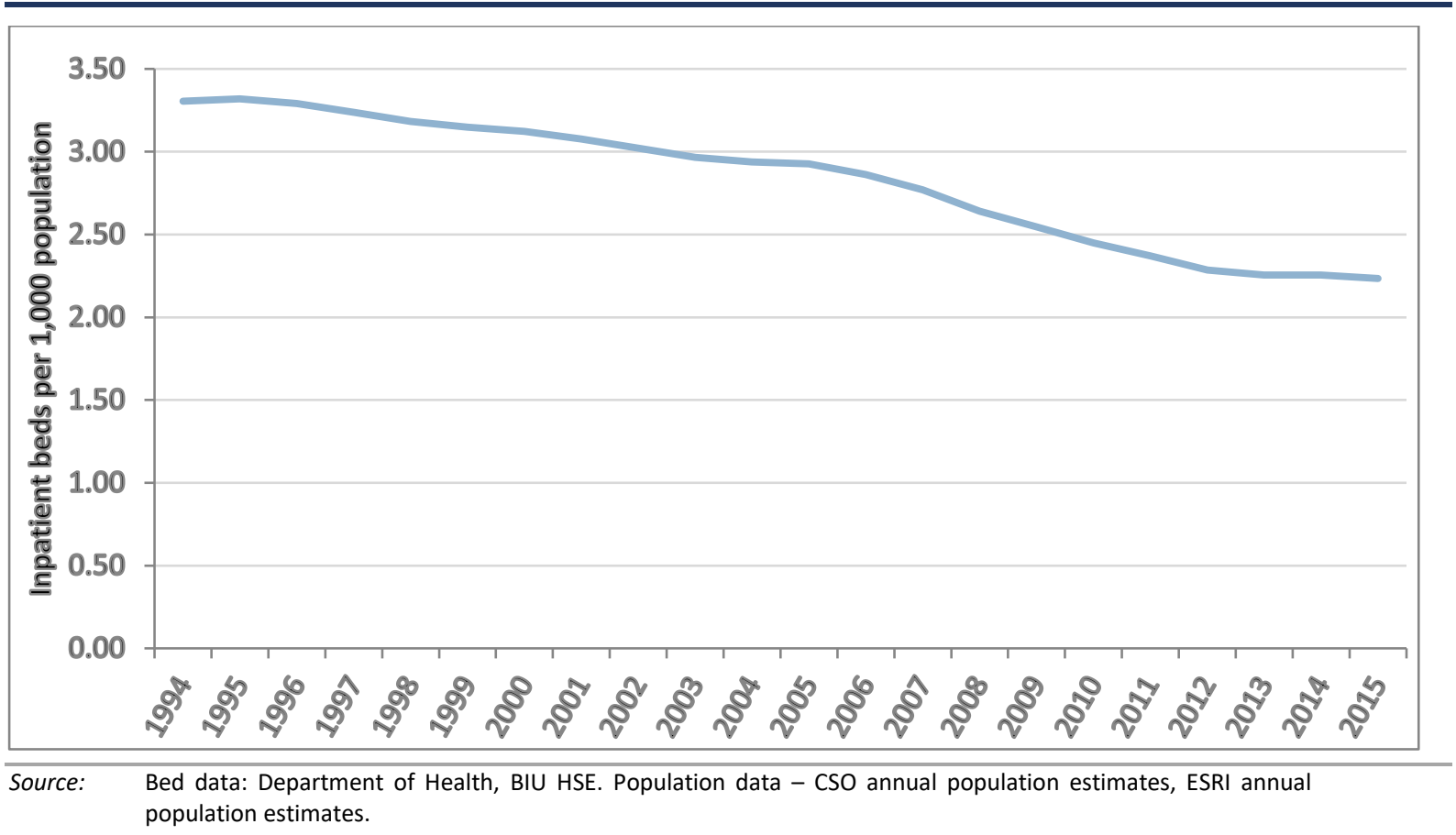

Figure A.1 in the appendix provides information on inpatient beds per 1,000 population aged 65 years and over between 2010 and 2015. Once more, a linear, but much steeper, decline is observed. Between 2010 and 2015, there was a 20.3 per cent reduction in inpatient beds for per 1,000 population aged 65 years and over. This dramatic reduction is in the context of those in this age group being high users of inpatient care (Wren et al., 2017).

\section{Inpatient bed supply across hospitals}

In this report, we are interested in the impact of changes in inpatient bed supply, across hospitals and over time, on inpatient LOS in Ireland. In order to examine these relationships, we accessed inpatient bed data from the Planning and Business Information Unit (BIU) in the HSE. These data provide the average monthly number of regularly maintained and staffed acute inpatient beds available for all acute public hospitals in Ireland between 2010 and 2015. These beds are a mixture of beds open on all days (7-day beds) and beds closed on some days such as at the weekend (5-day beds). Average capacity (beds available) in each month was calculated by summing all 7-day and 5-day acute beds in each hospital in every month, and dividing this number by days in said month. This ensures 5-day and 7day beds are not counted as equivalent in terms of the number of days they are available within a given month. These data are used as an average capacity measure by the HSE and the Department of Health and are provided to organisations including the OECD as reliable estimates of available hospital capacity in Irish public hospitals. In this report, we include inpatient bed data between January 2010 and December 2015 only. While the national number of inpatient beds is available for the pre-2010 years, as shown in Figure 3.1 and Figure 3.2, data at the hospital-month level are only available from 2010. 
In order to compare changes in inpatient bed supply across hospitals of different sizes, we constructed a standardised capacity variable for each hospital (Equation 3.1):

$$
B e d s_{-} S t_{h m}=\frac{B e d s_{h m}-\overline{B e d s}_{h}}{\sqrt{\frac{1}{72} \sum_{m=1}^{72}\left(B e d s_{h m}-\overline{B e d s}_{h}\right)^{2}}},
$$

where the mean number of inpatient beds available in each hospital $(h)$ over the whole period, $\overline{B e d s}_{h}$, was subtracted from the number of available inpatient beds in each of the 72 months in this period, Beds $s_{h m}$, (with $m$ signifying month) and subsequently divided by the standard deviation in bed availability in each hospital over the whole period. This provides us with a standardised variable with mean equal to 0 and a standard deviation equal to 1 . Examining inpatient bed supply using the standardised variable allows us to compare supply changes across different hospitals of different sizes and to take advantage of bed changes at the month level. It also assists ease of interpretation of the results in Chapter 5.

\subsubsection{Day patient bed supply}

While inpatient care and inpatient LOS are the focus of this report, day patient care is also an important component of the acute hospital sector. A day patient (day case) is a patient who attends an acute hospital for curative or medical treatment, but does not stay overnight. Often due to the shorter length of a stay per visit, a number of day patients use the same bed on a given day, with a 133 per cent occupancy rate estimated (Keegan et al., 2018a). In contrast to inpatient beds, the number of day patient hospital beds in Ireland has increased over time. Some of this increase has likely been due to the re-designation of inpatient beds to day patient beds in recent years, rather than a change in overall acute bed capacity, as there has been no substantial increase in new hospital infrastructure to explain the increases in day patient beds.

Figure 3.3 illustrates the total number of day patient beds in public hospitals in Ireland between 2003 and 2015 (data from before 2003 are not available) using data from the Department of Health (pre-2008) and the HSE. ${ }^{12}$ It is clear that, unlike inpatient bed supply, day patient bed numbers more than doubled between 2003 and 2012, before stabilising.

12 For the Department of Health data, see https://health.gov.ie/publications-research/statistics/statistics-bytopic/public-hospital-bed-numbers/. 


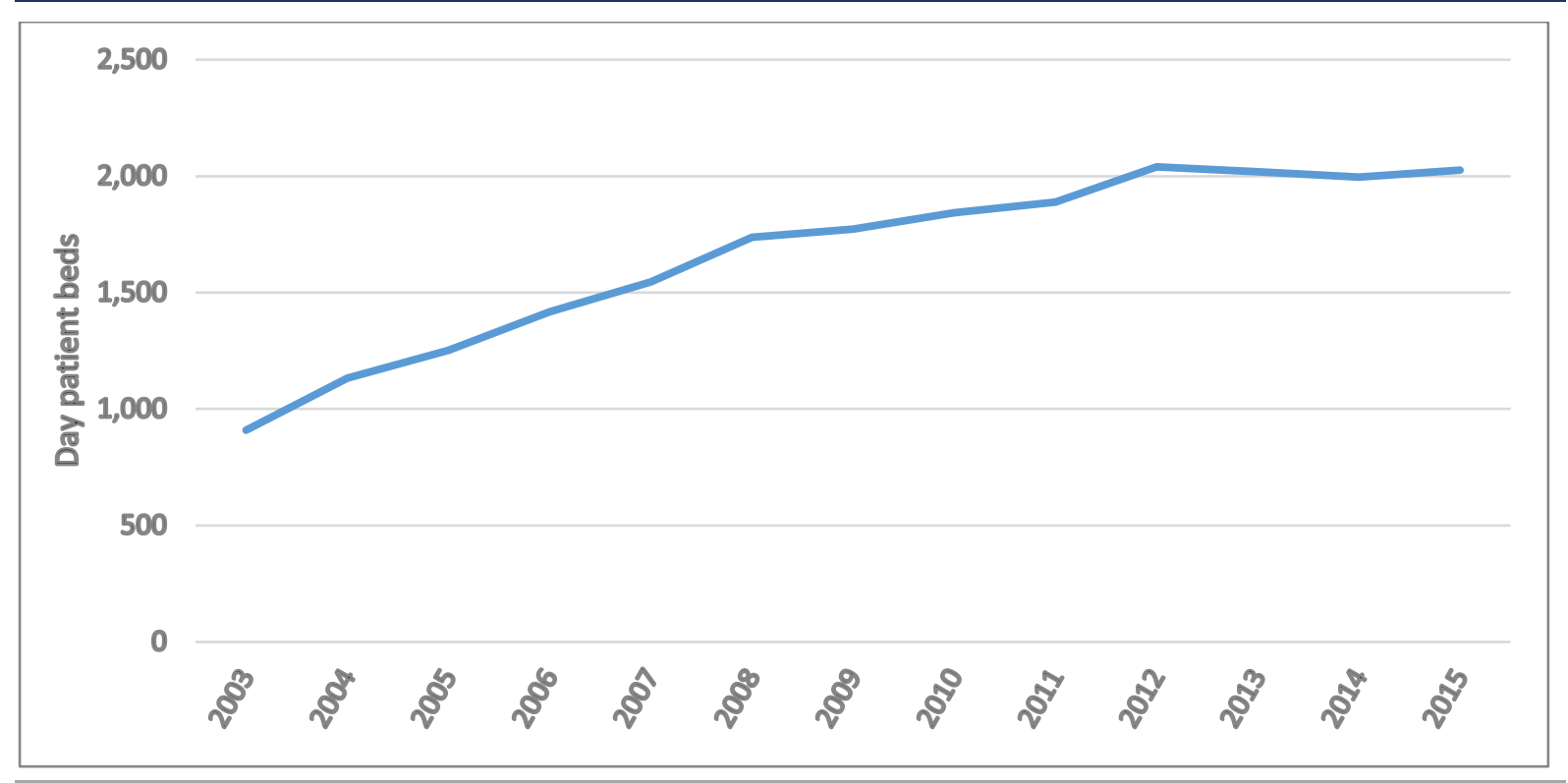

Source: Department of Health; BIU HSE.

Examining this increase in day patient beds on a per capita basis, Figure 3.4 illustrates the total number of day patient beds per 1,000 population in public hospitals in Ireland between 2003 and 2015. An increase in per capita numbers was observed between 2003 and 2012. However, per capita numbers actually fell between 2012 and 2015 as the population grew, though the absolute number of day patient beds remained static.

\section{FIGURE 3.4 DAY PATIENT BEDS PER 1,000 POPULATION IN PUBLIC HOSPITALS IN IRELAND, 2003-2015}

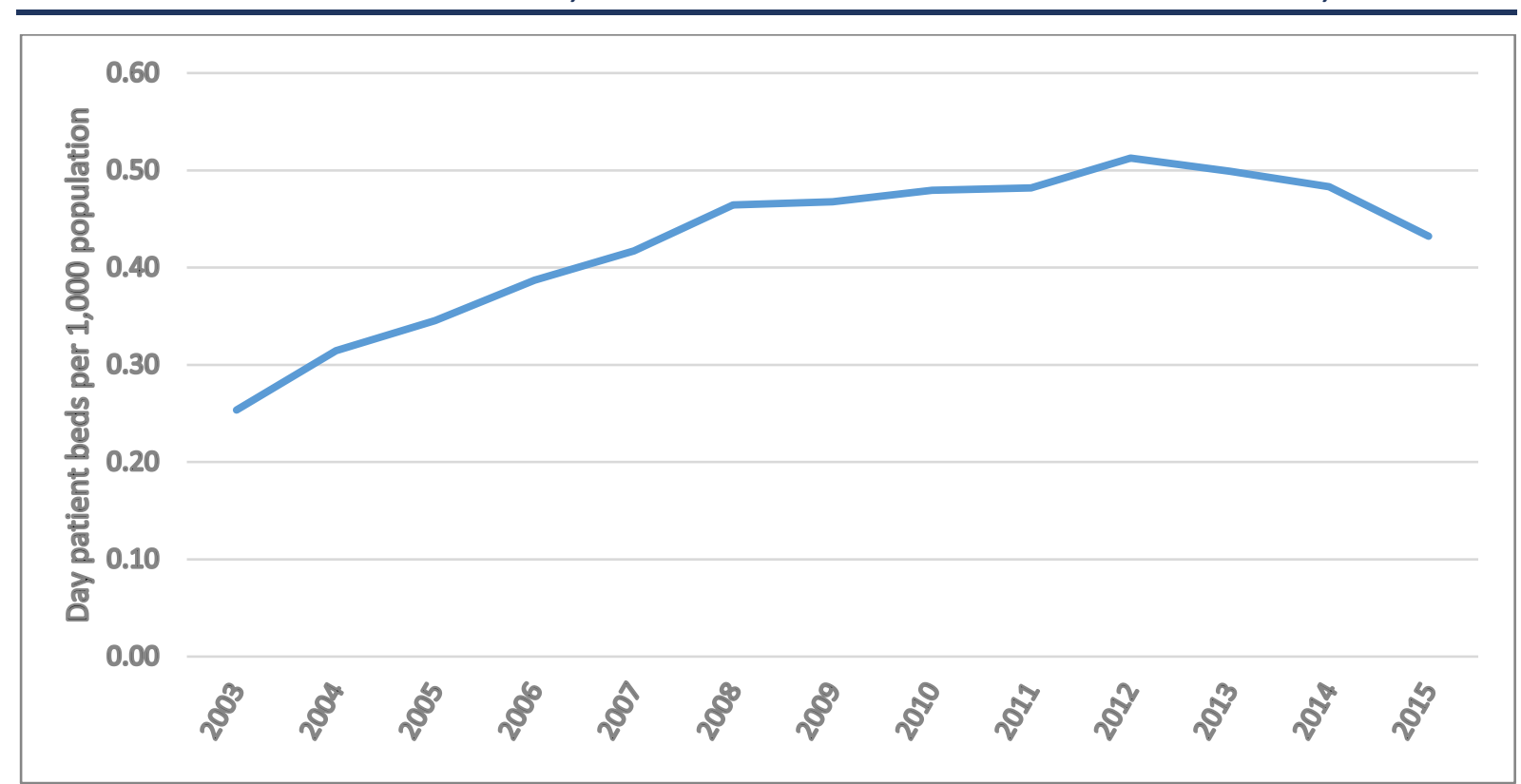

Source: Bed data - Department of Health, BIU HSE. Population data - CSO annual population estimates, ESRI annual population estimates.

Figure A.1 in the appendix provides information on day patient beds per 1,000 population aged 65 years and over between 2010 and 2015. During this time, there 
was a 6.5 per cent reduction in day patient beds for per 1,000 population aged 65 years and over.

\subsubsection{Private hospital beds}

Private hospital bed data are much more limited than data for public hospitals, partly because private hospitals do not report activity to HIPE. The number of private hospital beds is likely to have increased substantially over time as new private facilities have opened in Ireland, though the size of any increase is unknown. There were an estimated 1,975 private hospital inpatient beds in Ireland in 2015 (Wren et al., 2017), with approximately 16 per cent of all inpatient beds located in private hospitals.

In 2015, there were an estimated 946 day patient beds in private hospitals (Keegan et al., 2018a), corresponding to 32 per cent of all day patient beds in the acute hospital sector. These numbers emphasise that the private hospital system is a large component of the acute hospital sector. However, there is little information available about the composition of bed supply, such as number of beds across hospitals. Access to such information in the future would greatly expand the ability of researchers and policymakers to understand the acute hospital system in Ireland.

\subsection{INTERNATIONAL COMPARISONS}

In this section we use OECD data to compare inpatient bed supply in Ireland with that of other countries. As with all international comparisons, care should be taken when interpreting these numbers, as criteria for inclusion in OECD metrics does differ across countries (OECD, 2012).

\subsection{Inpatient beds per capita}

In 2000, inpatient bed supply per capita was already amongst the lowest in the OECD (Department of Health and Children, 2002). Supply in Ireland reduced further in subsequent years. Figure 3.5 illustrates that OECD data for 2015 indicate that Ireland ranked as the sixth lowest country in the OECD for inpatient bed supply, with 2.4 acute beds per 1,000 population. Slight variation in definitions of an inpatient bed explains the differences between these figures and those provided earlier in this report; in particular, OECD estimates include psychiatric care beds. 
FIGURE 3.5 ACUTE INPATIENT BED CAPACITY PER 1,000 PEOPLE ACROSS OECD COUNTRIES, 2015*

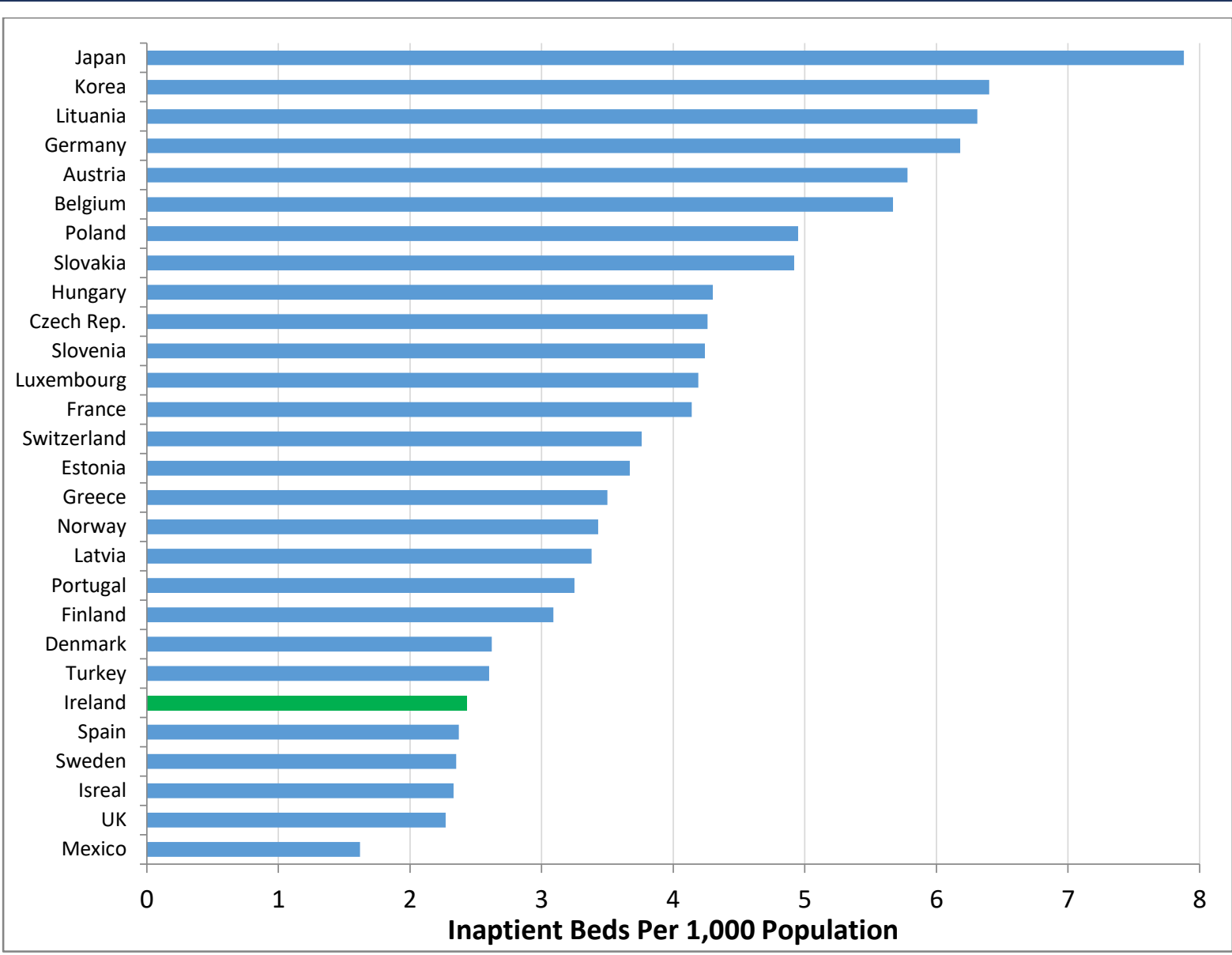

Source: OECD (2016).

Notes: $\quad *=$ or nearest year. Acute beds include beds used: for obstetrics; to cure non-mental illness or to provide definitive treatment of injury; to perform surgery; to relieve symptoms of non-mental illness or injury; to reduce severity of non-mental illness or injury; to protect against exacerbation and/or complication of non-mental illness and/or injury which could threaten life or normal functions; and perform diagnostic or therapeutic procedures (OECD, 2012).

The inpatient bed supply for Ireland in Figure 3.5 does not include a consistent estimate for beds in private hospitals. However, even accounting for inpatient bed supply in private hospitals, overall acute inpatient bed supply (including beds in both public and private hospitals) remains amongst the lowest in the OECD (Keegan et al., 2018a).

The OECD does not compare day patient beds per capita across countries. Comparing day patient bed supply across other countries (including the NHS in England) is more difficult due to differences in how acute and day patient beds are characterised.

\subsubsection{Inpatient bed occupancy rates}

Ireland has very high occupancy rates for inpatient beds. Occupancy rates measure, on average, the percentage of beds occupied by an inpatient at a moment in time. Internationally, an 85 per cent occupancy rate is used as a maximum threshold. This figure is often cited as a level above which concerns arise about patient safety (Bagust et al., 1999) and bed shortages (Madsen et al., 2014; 
The King's Fund, 2015). This maximum threshold has also been adopted by recent government bed capacity projections in Ireland (PA Consulting, 2018). The occupancy rate is also estimated as an average across the year, with large positive and negative fluctuations likely on a given day or within a hospital.

OECD data for 2000 indicate that Ireland experienced occupancy rates of less than 85 per cent (OECD, 2017a). However, occupancy rates in Ireland have steadily increased over time. Figure 3.6 presents average inpatient occupancy rates across OECD countries in 2015. The inpatient bed occupancy rates in Ireland was on average 94.7 per cent in 2015, the highest in the OECD, and almost 20 percentage points above the average of OECD countries included, and 10 percentage points about the maximum threshold after which concerns about patient safety arise.

In total, four countries have occupancy rates greater than 85 per cent. It is not surprising that three of these countries (Ireland, Israel, and the UK (England)) have amongst the lowest bed capacity rates in the OECD. ${ }^{13}$ In addition, as we discuss in Chapters 4 and 5, Ireland, England and Canada also have significant issues with delayed discharges. In this context, high occupancy is largely a consequence of low bed capacity.

13 While Denmark has been shown to have high occupancy rates (Madsen et al., 2014). This information is not provided to the OECD. 


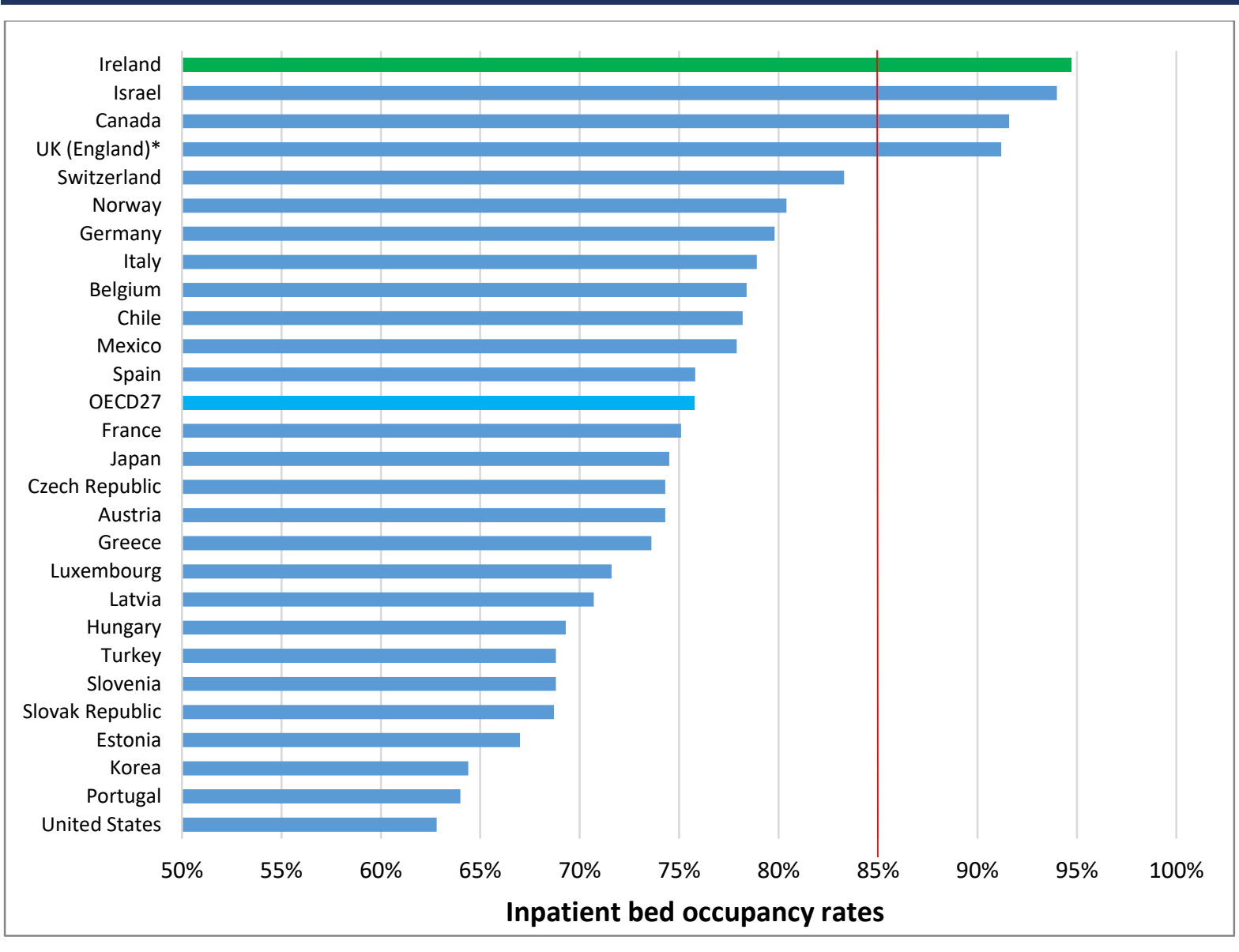

Note: $\quad *=$ or nearest year.

Source: OECD (2017).

\subsubsection{Projected bed capacity requirements}

The acute public hospital capacity constraints in Ireland have been acknowledged by the Government as a matter of concern, and there is a recognition that increases in inpatient bed supply in public hospitals are required. In 2018, a report authored by PA Consulting and funded by the Department of Health referred to the necessity to increase inpatient bed capacity considerably. The report found that by 2031, compared with bed supply in 2015, an additional 2,600-7,150 beds will be required in public hospitals (PA Consulting, 2018). The large range in projections is largely dependent upon assumptions around future improvements to non-acute supply and accompanying substitution effects between hospital and non-acute care. The largest projected increases were estimated for inpatient care, with an estimated additional 2,100-5,800 inpatient beds required by 2031 (PA Consulting, 2018). Importantly, these estimates assume a maximum 85 per cent occupancy rate threshold. ${ }^{14}$ The National Development Plan has now reemphasised the need for additional beds, and it includes plans to include 2,600 additional acute public hospital beds by 2027 (Government of Ireland, 2018a). This equates to the lowest number in the PA Consulting range, which assumes considerable investment in

14 In order to achieve 85 per cent occupancy rates in the base year (2015), ceteris paribus, 933 additional inpatient beds would be required in that year. 
non-acute supply and the ability to substitute many services from the acute hospital sector. The National Development Plan also sets out plans to develop three elective-only hospitals in Dublin, Cork and Galway.

A recent study found that an additional 3,200-5,600 hospital beds will be required by 2030 based upon projected demand increases in the system (Keegan et al., 2018a). Partitioned by bed type, the study estimated the need for an additional 2,620-4,430 inpatient beds and an additional $610-670$ day patient beds by 2030 , as compared to 2015 levels. The lower projected bed requirements in the study are again dependent upon improvements being made to non-acute supply and substitution towards non-acute care where appropriate; they assume 85 per cent occupancy rates are achieved by 2030 (Keegan et al., 2018a). These findings also imply that the extra 2,600 total beds outlined in the National Development Plan are unlikely to be sufficient to meet demand for inpatient care, even in the context of large substitution of care towards the non-acute sector.

While the projected number of added hospital beds required by 2030 is high in absolute terms, Keegan et al. (2018a) highlight that even for the highest projected level of expansion, public hospital inpatient beds per capita would not exceed 3 per 1,000 population and Ireland would remain close to the bottom of OECD countries in line with Figure 3.5. However, failure to increase capacity would result in public hospital inpatient beds per capita falling below 2 per 1,000 population, which would be by far the lowest in the OECD based upon 2015 figures (Keegan et al., 2018a).

\subsection{GEOGRAPHIC PATTERNS IN THE SUPPLY OF PUBLIC HOSPITALS}

The previous sections briefly describe the acute hospital sector in Ireland, the supply of inpatient and day patient hospital beds over time and how Ireland compares to international peers in terms of hospital bed supply. This section discusses the distribution of acute hospital care across Ireland and examines geographic catchment areas of hospitals. It follows a similar template to that used in Smith et al. (2019) to examine the geographic variation in non-acute supply in Ireland. Much of the information in this section is derived from the HIPE dataset. In accordance with this project's data-sharing agreement with the HPO, in order to prevent disclosure of hospitals, the names of specific hospitals and areas of residence of patients are not identified.

\subsubsection{Locations of acute public hospitals in $\mathbf{2} 015$}

Figure 3.7 presents the location of each of the 29 hospitals in 2015 with a Tier 1 ED, as mapped by the Irish Association of Emergency Medicine (IAEM). The historical pattern of having a hospital located in every county can still be seen, and a large acute public hospital still exists in most counties. Six hospitals are located in Dublin (three in Dublin North and three in Dublin South), while Cork has 2 large 
hospitals both located in Cork City. Of the 7 counties in 2015 without a Tier 1 ED public hospital, 3 (Monaghan, Clare, Roscommon) had their local Tier 1 ED reconfigured between 2005 and 2015. A further 6 areas (Tipperary North, southern and northern Cork county, northern Louth county, and Wicklow) also saw their 'local' ED reconfigured from Tier 1 ED status in recent years.

FIGURE 3.7 LOCATION OF ACUTE PUBLIC HOSPITALS WITH TIER 1 EMERGENCY DEPARTMENT IN IRELAND

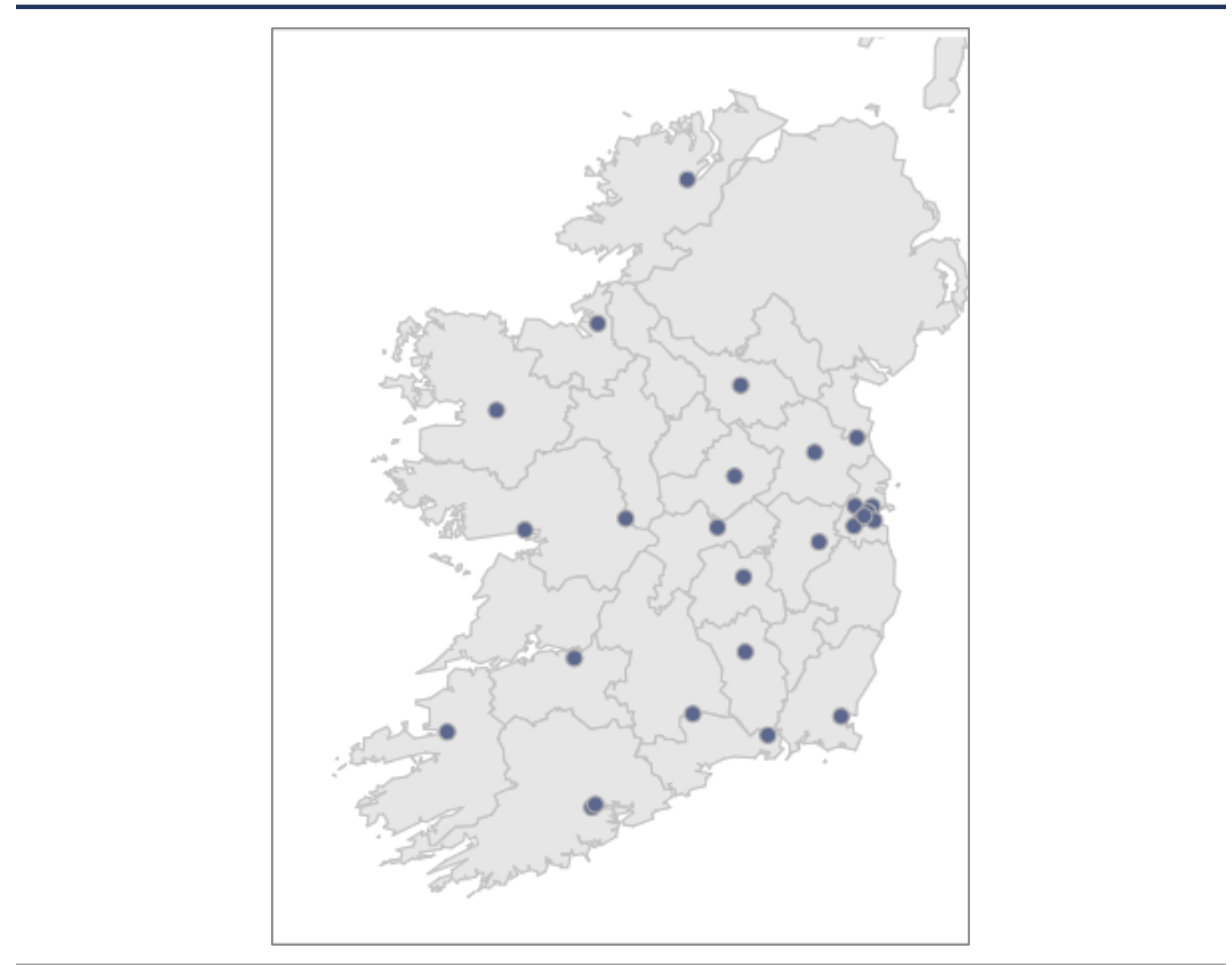

Source: Irish Association for Emergency Medicine; see http://www.iaem.ie/public/irish-emergency-departments/.

Table 3.2 presents the proportion of emergency inpatient discharges that occurred in the larger hospitals with a Tier $1 \mathrm{ED}$ and other smaller hospitals, many of whom were reconfigured, between 2005 and 2015. Overall, it is clear that the 29 larger hospitals provide the majority of emergency inpatient care, with the proportion provided there having increased to over 90 per cent in 2015. 
TABLE 3.2 PROPORTION OF EMERGENCY INPATIENT DISCHARGES IN TIER 1 ED AND OTHER PUBLIC HOSPITALS IN IRELAND, 2005-2015

\begin{tabular}{|l|c|c|}
\hline & Tier 1 ED hospitals* & Other hospitals \\
\hline 2005 & $81.9 \%$ & $18.1 \%$ \\
\hline 2006 & $83.1 \%$ & $16.9 \%$ \\
\hline 2007 & $84.5 \%$ & $15.5 \%$ \\
\hline 2008 & $85.4 \%$ & $14.6 \%$ \\
\hline 2009 & $85.8 \%$ & $14.2 \%$ \\
\hline 2010 & $89.3 \%$ & $10.7 \%$ \\
\hline 2011 & $90.8 \%$ & $9.2 \%$ \\
\hline 2012 & $90.9 \%$ & $9.1 \%$ \\
\hline 2013 & $92.5 \%$ & $7.5 \%$ \\
\hline 2014 & $91.4 \%$ & $8.6 \%$ \\
\hline 2015 & $90.3 \%$ & $9.7 \%$ \\
\hline
\end{tabular}

Notes: $\quad *=29$ hospitals listed as having a Tier 1 ED in 2015 in Table 3.1.

\subsubsection{Hospital reconfiguration}

There was a substantial reconfiguration of the acute public hospital sector in recent years. A number of hospitals had their ED and emergency care services reduced or removed, and since 200510 hospitals had their EDs reconfigured from largely operating 24 hours a day, 7 days a week, to a more limited ED or injury unit. A full list of changes and when they occurred can be found in Table A.2.

Reconfiguration of services occurred, in part, following investigations of hospitals by the Health Information and Quality Authority (HIQA) where quality of care concerns arose (HIQA, 2009; 2011). Following these reports, the HSE identified a number of smaller hospitals where significant changes in the role and scope of the services (in particular emergency and inpatient services) they provided was to be reconsidered (HSE, 2012). This resulted in the reconfiguration of services in those hospitals identified in the HSE report (one hospital listed saw services reestablished subsequently). Recent qualitative evidence using interviews with stakeholders involved in reconfiguration found that local context and politics can influence decisions about reconfiguration of hospital services (McHugh et al., 2019).

While hospital reconfiguration was one of the most substantial changes to the acute hospital system in recent years, there is little information or research on the outcomes of the reconfiguration. For example, there is no study examining the impact reconfiguration had on patient outcomes or overall mortality. Recent studies have examined the impact reconfiguration had on the regional distribution of acute services (Droog et al., 2018; McHugh et al., 2019). The evidence shows that the reconfiguration mainly affected the southern, western, mid-western, and north-eastern regions (Droog et al., 2018; McHugh et al., 2019). Complex emergency care was centralised to larger hospitals in Dublin, Cork, Galway and 
Limerick. The changes to hospital services mean that, in 2016, Tier 1 EDs per capita differed considerably across areas; ranging from 1 per 128,000 population in the south-east region (Carlow, Kilkenny, Wexford, Waterford and Tipperary South) to 1 per 385,000 population in the mid-west region (Limerick, Clare and Tipperary North) (Droog et al., 2018). Higher relative supply was also identified in Dublin more generally.

In the context of the findings of Droog et al. (2018), Figure 3.8 illustrates the impact reconfiguration of hospital services had on access, as measured by drive time, to Tier 1 EDs across the country. The figure combines Tier 1 ED locations with maps of the road network across Ireland in 2005, 2010 and 2015, and geographic location presented at Census electoral division level. The graph shows the proportion of electoral divisions that fell within 0-15 minute, 16-30 minute and 31 minute and over drive times of a Tier 1 ED in Ireland, in each of the years examined. The colour green represents shorter travel times (0-15 minute drive), while red represents longer travel times ( $31+$ minute drive) to an ED for people living in each electoral division.

It is clear that many areas, particularly along the western and southern coasts, have journey times greater than 30 minutes. This is largely a consequence of the rurality of these areas. However, the graph also shows that the share of areas with distances greater than 30 minutes has increased over time. The reconfiguration had particular impacts on counties Cork, Tipperary, Roscommon and Longford as they saw the greatest increase in long journey times to Tier 1 EDs. Note, however, that these maps do not capture population density. Therefore, while Droog et al. (2018) showed that the south-east region has a greater density of EDs per capita, average drive times to the nearest Tier 1 ED in counties Wicklow, Carlow and Wexford are relatively long. 
FIGURE 3.8 DRIVE TIME TO A TIER 1 EMERGENCY DEPARTMENT, 2005, 2010 AND 2015

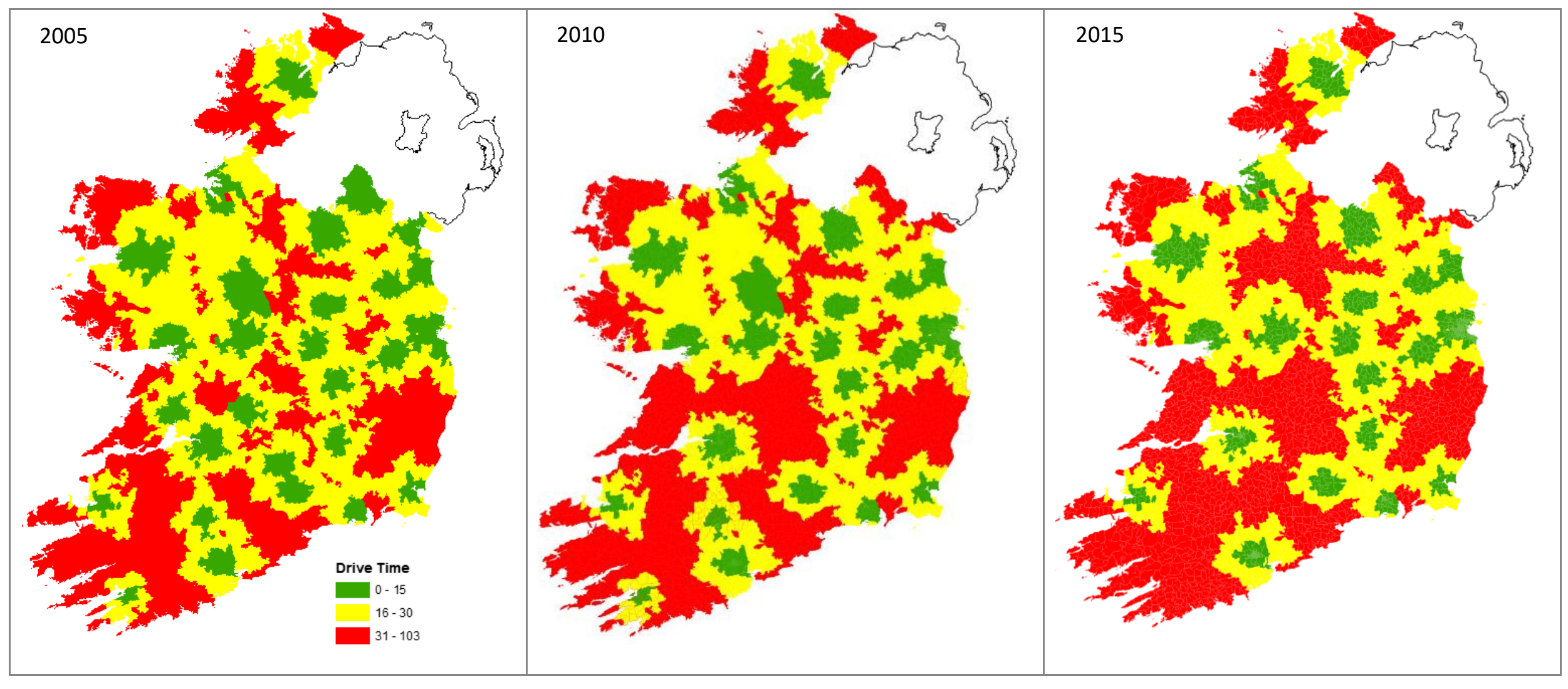


It should be noted that despite a large reconfiguration of public hospital services in Ireland in recent years, as compared to other countries Ireland still has a relatively large supply of public hospitals per capita. The 29 Tier 1 EDs in Ireland cater for a population of 4.78 million, which equates to 1 per 165,000 population. In the English NHS, which has also seen significant reconfiguration and consolidation of hospitals services in recent years (Collins, 2015), there are 176 equivalent Type 1 'accident and emergency' departments for a population of 54.5 million, equating to 1 per 310,000 population. ${ }^{15}$ While comparing hospitals across countries is difficult, especially with different healthcare systems in place, Ireland has a relatively large supply of acute care hospitals, and this increases further when private hospital supply is included. A previous study argued that the large number of hospitals in Ireland, relative to population size, may impact the efficiency of the hospital sector in Ireland (Gannon, 2005), though consolidation and mergers may intensify geographic variation in acute care access.

\subsubsection{Acute public hospital catchment areas}

There are no official catchment areas for public hospitals in Ireland; in other words, individuals are not required to visit a specific hospital based upon their address. However, we find that catchment areas defined by location and distance to hospital do exist in practice. In this section, we use information from HIPE to examine the proportion of patients in area of residence (Resid), using each hospital in the data, and vice versa.

Within HIPE, discharges are grouped into 49 Resids, based upon their address. These areas of residence relate to the patient's county of residence, except in Dublin where they relate to patient postcodes. Using this information, we match patients from each Resid to the hospital in which they receive care, and estimate the proportion of discharges in each Resid who use a specific hospital. We then sort hospitals according to their 'dominance', which means that for each Resid, we characterise the hospital with the largest number of discharges as the most dominant hospital, the hospital with the second largest of discharges as the second most dominant, and so on. We present this information in Figure 3.9. The most dominant hospital for a Resid (the hospital with the largest number of discharges from that Resid) is illustrated in the colour blue, with the second and third most dominant hospitals illustrated in orange and green respectively, and all other hospitals used illustrated in red.

Figure 3.9 shows that many areas have a single dominant hospital; virtually all ED inpatient care accessed by people within a single area occurs within one hospital. But that is not always the case. For example, in Resid 18 no hospital clearly dominates, and inpatients from this area use at least four hospitals in large

15 See https://publications. parliament.uk/pa/cm201617/cmselect/cmhealth/277/277.pdf. 
numbers. Overall, in approximately one-third of Resids, the most dominant hospital provides just over 50 per cent of care. Results are similar within and outside of Dublin (see Figure A.2).

\section{FIGURE 3.9 PROPORTION OF EMERGENCY INPATIENT DISCHARGES IN EACH AREA OF RESIDENCE ACROSS HOSPITALS}

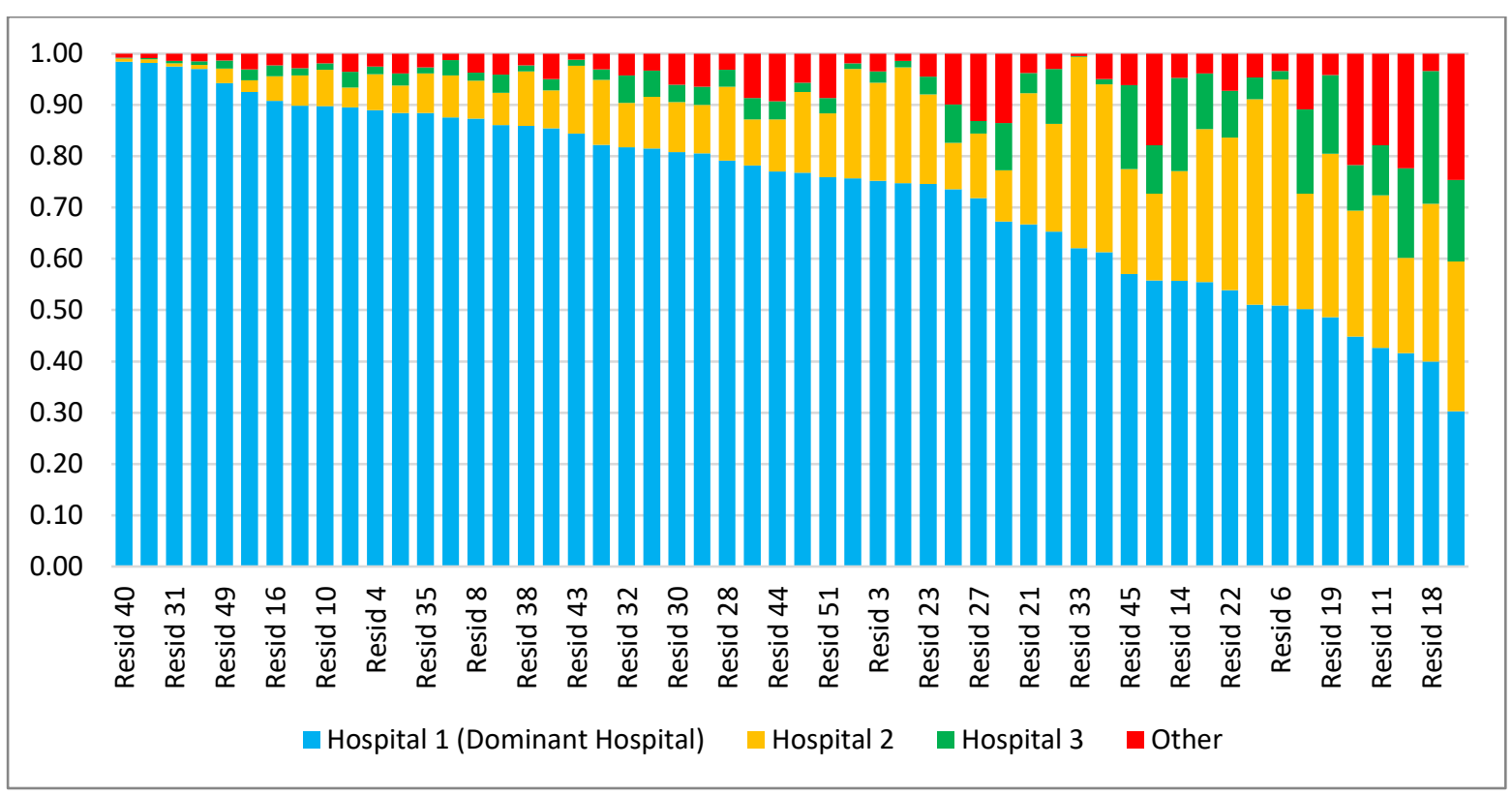

Notes: $\quad$ Analysis based on emergency department inpatient activity in 2015, from HIPE data.

The location of a hospital is the main factor driving its use. Removal of a hospital, especially the most dominant hospital, from a region is likely to have consequences for a number of neighbouring hospitals. In Figure A.3, we detail a short case study on three areas where the most dominant hospital for each area saw its ED services reconfigured between 2005 and 2015. The data suggest that the dispersion of emergency care services following the removal of ED care from the most dominant hospital impacted utilisation in at least three neighbouring hospitals in each case.

\subsection{HOME CARE}

This section outlines the data on home care in Ireland, changes to supply in recent years and differences in supply across regions in Ireland. In these analyses, home care relates to formal home care financed by the State. Informal home care provided by a spouse, family member or neighbour remains the predominant form of home care provided in Ireland (Hanly and Sheerin, 2017; Kiersey and Coleman, 2017), but it is not included in these analyses.

\subsubsection{Definition of home care}

Home care relates to domestic, personal or health care provided to individuals in their own home. Home care is used to allow persons with special care or health needs to stay in their home, and it is expected to have the additional benefit of 
reducing the need for individuals to use other services such as acute hospital care and LTRC. Home care services provided may be quite broad but, in general, home care is designed to aid activities of daily living (ADLs) such as personal hygiene, dressing, eating and mobility; personal health care may also be provided, such as replacing bandages and ensuring medication adherence. Home care is generally targeted at older individuals in the population. However, some home care, especially care provided through disability services, is also provided to younger individuals with a disability or illness.

Home care supply, financing and utilisation differ considerably across countries. Home care in Ireland is dominated by informal caregiving, following a family-based structure similar to southern European countries (Hanly and Sheerin, 2017; Ilinca et al., 2015) and no statutory formal home care scheme exists currently (Kiersey and Coleman, 2017). The Sláintecare Implementation Strategy states that a statutory home care scheme will be enacted in 2021, though no details on supply, assessment criteria or co-payment requirements so far exist. The provision of formal home care was included in legislation for the first time in the 1970 Health Act, which established the first home help scheme (Murphy et al., 2015) with service provision at the discretion of the local health authorities (Timonen Doyle, 2008). Publicly financed home care lies under the remit of the Social Care Division of the HSE.

Approximately 73 per cent of home care use is publicly financed and provided by a mixture of state-run, not-for-profit and for-profit organisations (Wren et al., 2017). Privately purchased home care (27 per cent of use) is less regulated in terms of the quality of the service (Timonen et al., 2012), though may be more flexible in meeting patients' demands (Timonen and Doyle, 2007). Eligibility for public home care is predominantly for those aged 65 years and over who are assessed on care needs and informal care supply. The home care schemes in place in Ireland have all stated that income is not assessed, and the care is provided free of charge to the client. However, some (Timonen and Doyle, 2007) have questioned whether in practice care was always provided free to recipients; they argue that there is evidence that in some areas medical card coverage was used as part of the assessment and that some clients were charged a co-payment for care. ${ }^{16}$

As a consequence of the recent recession in Ireland, home care saw large expenditure cuts between 2008 and 2014. In this context, the $€ 390$ million budget allocation for home care in $\mathbf{2 0 1 7}$ was similar to the amount spent on home care in 2008 , despite a significant increase in the older population during the intervening period. ${ }^{17}$ In 2017, 2.75 per cent of the HSE gross non-capital total expenditure was

16 Income means-tested medical cards entitling free primary, community and acute care, covering 36 per cent of the population (Department of Health, 2017a).

17 Between 2006 and 2015 the population aged $65+$ increased by 141,000 (30 per cent). 
spent on home care. This equates to less than half of the amount spent on the LTRC Fair Deal scheme, though the numbers using home care are higher. Between 2006 and 2018, two separate public home care schemes existed: the home care package (HCP) scheme and the home help scheme. Historically, the home help scheme provided domestic support including assistance with cleaning, cooking and basic household tasks, while the HCP scheme was introduced in 2006 to provide more intensive care to allow for older people, particular those discharged from hospital or from a rehabilitation facility, to be cared for in their own home. While differences between the two schemes existed in the past, in recent years they have provided similar care and support to aid individuals at home (Care Alliance Ireland (2018)) and in 2018 were merged into the Home Support Scheme.

When generating a metric for home care spending in this report, we combine home care hours provided across both the HCP scheme and the home help scheme. This is necessary due to the practical overlap between the schemes, but it does have the effect of smoothing away some of the actual variation in the mix of services provided that might have existed across areas. This is probably less of an issue for recent years, and combining the spending from the two programmes also means the activity covered by our home care variable is very similar to the care provided in the new Home Support Scheme.

Note that we do not examine the recently introduced intensive home care packages (IHCPs). These packages were introduced to target specific high healthcare demand patients, ${ }^{18}$ such as those with dementia (Keogh et al., 2018), and in general provide a substantial level of support to only a small number (fewer than 300 in 2015) recipients.

\subsubsection{Home care data}

Due to the lack of information on privately-purchased home care at a regional level, we examine only state-funded home care in this project. Home care data on publicly financed home care supply, between 2012 and 2015, were provided by the HSE Social Care Division. These data capture the number of home care hours provided by the home help scheme and the number of recipients covered by the HCP scheme (with less information on hours) across the 32 Local Health Offices (LHOs - the most granular geographic level for which data are available). These data are available at the month level, allowing us to construct an area-month home care supply variable. 
We merge information from the HCP and home help schemes to estimate an overall home care hours supply per capita variable for each LHO using the following steps. First, we estimate the number of home care hours provided annually in an average HCP, using HSE home care data from 2017. This analysis yields an average of 300.6 hours per HCP per annum. Second, we combine the estimated home care hours provided as part of a HCP with hours provided as part of the home help scheme. To account for differences in population size across areas, we construct a 'home care hours per population aged 65 years and over' measure for each LHO, by dividing the estimated hours by the population aged 65 years and over in each respective LHO and year. The home care supply variable used is outlined in Equation 3.2:

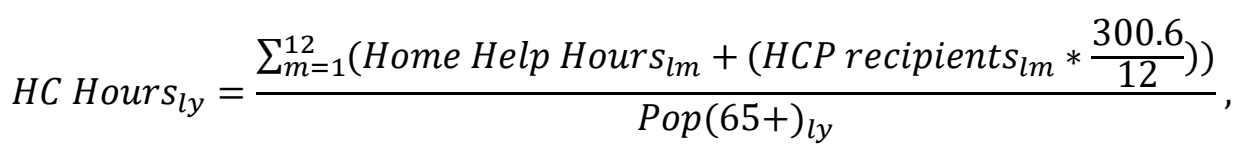

where / represents the LHO, $\mathrm{m}$ the month, and $y$ the year.

To aid consistency with Smith et al. (2019), we estimate supply at the county level between 2012 and 2015 in the following sections using the apportioning approach in Smith et al. (2019) to express differences in home care supply across counties.

Due to the varying definitions of home care used across countries, it is not possible to compare the supply of home care in Ireland to that in other countries.

\subsubsection{Trends over time in home care hours}

Smith et al. (2019) illustrated the distribution of the annual average number of home care hours per person aged 65 years and over in Ireland in 2014, finding that there was significant geographic variation. In Figure 3.10 and Table 3.3, we expand upon the previous report and illustrate supply across counties over time. Once more, large geographic variations are observed. Counties such as Sligo, Leitrim, Kerry, Louth, Meath and Donegal have consistently higher per capita home care hours across all years. Significant differences within counties are also observed over time. Some counties, including Dublin North, Louth, Meath, Clare and Laois, saw large increases in hours relative to the older population between 2012 and 2015. In the same period, other counties, including Longford, Westmeath, Waterford and Cork, saw reductions in home care hours relative to population.

The Gini coefficient provides information on the unequal distribution of supply across counties, where a value of 0 equates to perfect equality in supply, a number greater than 0 denotes inequality in supply, and 0.1 used as a rule of thumb for large inequalities. In Table 3.3, the Gini coefficient for the distribution of home care hours is larger than 0.1 in each year examined, indicating an unequal distribution 
across areas. The Gini coefficient also increased from 0.118 in 2012 to 0.125 in 2015. ${ }^{19}$

FIGURE 3.10AVERAGE ANNUAL NUMBER OF PUBLICLY FINANCED HOME CARE HOURS PER PERSON AGED 65+ BY COUNTY, 2012-2015

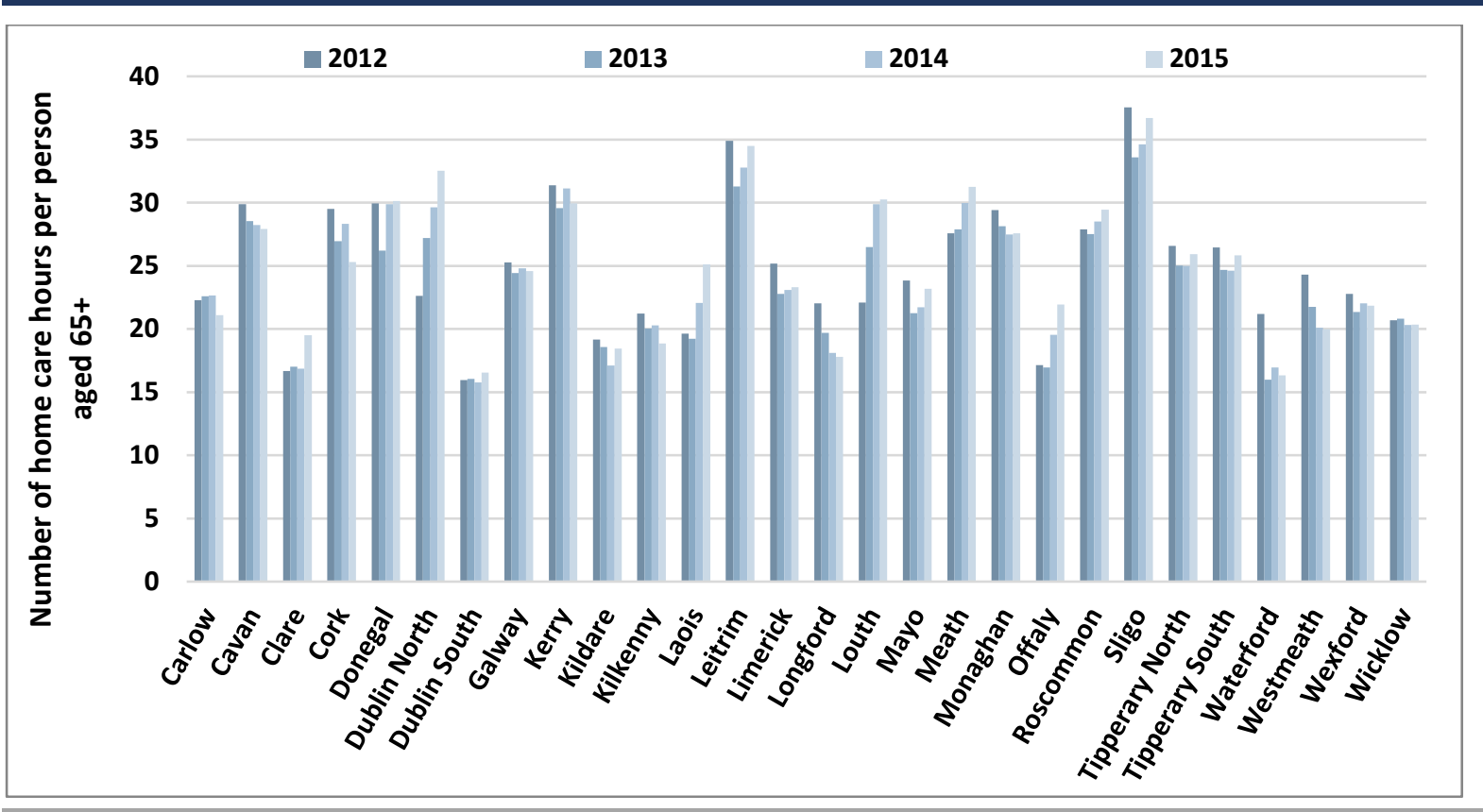

19 Gini coefficients estimated using the conindex command in Stata (O'Donnell et al., 2016). 
TABLE 3.3 AVERAGE ANNUAL NUMBER OF PUBLICLY FINANCED HOME CARE HOURS PER PERSON AGED 65+ BY COUNTY, 2012-2015

\begin{tabular}{|c|c|c|c|c|c|}
\hline Geographic area & 2012 & 2013 & 2014 & 2015 & $\%$ change $2012-2015$ \\
\hline Carlow & 22.3 & 22.6 & 22.7 & 21.1 & -5.29 \\
\hline Cavan & 29.9 & 28.5 & 28.2 & 27.9 & -6.48 \\
\hline Clare & 16.7 & 17.0 & 16.9 & 19.5 & 17.00 \\
\hline Cork & 29.5 & 27.0 & 28.3 & 25.3 & -14.25 \\
\hline Donegal & 30.0 & 26.2 & 29.9 & 30.1 & 0.54 \\
\hline Dublin North & 22.6 & 27.2 & 29.6 & 32.5 & 43.81 \\
\hline Dublin South & 15.9 & 16.0 & 15.8 & 16.6 & 3.79 \\
\hline Galway & 25.3 & 24.4 & 24.8 & 24.6 & -2.81 \\
\hline Kerry & 31.4 & 29.6 & 31.1 & 30.0 & -4.55 \\
\hline Kildare & 19.2 & 18.6 & 17.1 & 18.5 & -3.61 \\
\hline Kilkenny & 21.2 & 20.0 & 20.3 & 18.8 & -11.18 \\
\hline Laois & 19.6 & 19.2 & 22.1 & 25.1 & 27.88 \\
\hline Leitrim & 34.9 & 31.3 & 32.8 & 34.5 & -1.14 \\
\hline Limerick & 25.2 & 22.8 & 23.1 & 23.3 & -7.40 \\
\hline Longford & 22.0 & 19.7 & 18.1 & 17.8 & -19.26 \\
\hline Louth & 22.1 & 26.5 & 29.9 & 30.3 & 37.03 \\
\hline Mayo & 23.8 & 21.3 & 21.7 & 23.2 & -2.76 \\
\hline Meath & 27.6 & 27.9 & 30.0 & 31.3 & 13.33 \\
\hline Monaghan & 29.4 & 28.1 & 27.5 & 27.6 & -6.27 \\
\hline Offaly & 17.1 & 17.0 & 19.5 & 21.9 & 27.94 \\
\hline Roscommon & 27.9 & 27.5 & 28.5 & 29.4 & 5.61 \\
\hline Sligo & 37.6 & 33.6 & 34.6 & 36.7 & -2.28 \\
\hline Tipperary North & 26.6 & 25.0 & 25.0 & 25.9 & -2.49 \\
\hline Tipperary South & 26.4 & 24.7 & 24.6 & 25.8 & -2.37 \\
\hline Waterford & 21.2 & 16.0 & 17.0 & 16.3 & -22.84 \\
\hline Westmeath & 24.3 & 21.8 & 20.1 & 20.0 & -17.89 \\
\hline Wexford & 22.8 & 21.3 & 22.0 & 21.8 & -4.14 \\
\hline Wicklow & 20.7 & 20.8 & 20.3 & 20.3 & -1.75 \\
\hline Ireland & 23.9 & 23.3 & 24.1 & 24.5 & 2.63 \\
\hline Gini Coefficient (aged 65+) & 0.118 & 0.113 & 0.101 & 0.125 & - \\
\hline
\end{tabular}

Source: Social Care Division, HSE.

\subsection{LONG-TERM RESIDENTIAL CARE}

This section discusses the data on LTRC care in Ireland, changes in LTRC bed supply in recent years and differences in LTRC bed supply across counties in Ireland.

\subsubsection{Definition of long-term residential care}

LTRC relates to personal or non-acute health care provided to individuals within a residential care setting over a sustained time period. LTRC is required when it is no longer possible for individuals to be cared for in their own home and the care needed does not require a stay in an acute hospital. Care provided in LTRC is often based around convalescence rather than medical treatment. In this report, LTRC 
bed data encompasses care beds required for varying durations, and therefore includes beds used for respite, rehabilitation, convalescence and palliative care. This is in line with recent analyses of LTRC in Ireland by Wren et al. (2017). ${ }^{20}$

Approximately 4 per cent of all those aged 65 and older are LTRC residents (Wren et al., 2017). While approximately 75 per cent of LTRC residents are covered under the statutory Nursing Home Support Scheme (NHSS), some residents are privately financed or funded under HSE legacy schemes (Wren et al., 2017).

The definition, use, delivery and financing of LTRC differs across countries. In 2015, the HSE spent (non-capital) €968 million on LTRC (Department of Health, 2017b), with the majority of expenditure spent on Fair Deal. A needs assessment is required to qualify for Fair Deal, with a co-payment required from each resident based upon a financial assessment of assets, such as the resident's home (for the first three years of their LTRC stay) and income. Up to 80 per cent of income and up to 7.5 per cent per annum of assets valued greater than $€ 36,000$ may be contributed by the resident. ${ }^{21}$

Similar to home care, LTRC is provided by a mixture of publicly-owned, voluntaryowned and for-profit centres. Under Fair Deal, individuals have an option to choose from all approved centres, regardless of whether the centre is run by the HSE or not. The majority of LTRC centres in Ireland are privately-owned, with the proportion of such centres increasing. In this report, we concentrate on the use of LTRC for older people (those aged 65 and over). In 2015, only 4.5 per cent of LTRC residents in centres designed to predominantly provide LTRC for older people (as opposed to those caring for younger people with disabilities) was provided to those aged under 65 years (Wren et al., 2017). ${ }^{22}$

\subsubsection{Long-term residential bed data}

In this report, LTRC beds per capita are estimated for each region in Ireland over time. There is no definitive list of LTRC centres or beds per centre available. In order to capture all LTRC, we use a number of different data sources. The most comprehensive data on LTRC bed supply was provided by the Health Information and Quality Authority (HIQA). The Health Act (2007) states that all LTRC centres

20 Wren et al. (2017) used the definition of LTRC applied by the Department of Health in its annual Long-stay activity statistics reports. The Department of Health distinguishes between long-stay beds and limited-stay beds. Long-stay beds include those for: extended/continuing care for people who have been assessed as being in need of long-term care; psychiatry of old age, for specialised psychiatric services; and 'young chronic sick' for young people with a longlasting illness that is usually irreversible and may be progressive. Limited-stay beds include beds for rehabilitation or convalescence after an illness/injury; palliative care for patients at a time 'when the medical expectation is no longer cure'; and respite, for 'the planned admission of dependent persons for short periods of time in order to assist carers in their task of caring'.

21 Contribution deferment ('Nursing Home Loan') is possible to be collected posthumously from the resident's estate.

22 The proportion aged $<65$ years is much higher when all centres providing care for people with physical and intellectual disabilities are included. 
must register with HIQA and HIQA is responsible for the regulation of designated centres for older people. Since 2010, HIQA have maintained a database of LTRC centres (both public and private) operating in Ireland. HIQA provided these data for the years 2012-2015 inclusive for this report. The HIQA data provide information on the centre's ID, provider (HSE, voluntary, or private), address of the centre, most recent registration date and the number of beds the centre has registered for occupancy.

The HIQA bed registry is not an exhaustive list, however, with a number of centres missing in earlier years. To fill some of the gaps, we also examined the Department of Health's long-stay activity statistics (LSAS) and the Nursing Homes Ireland (NHI) surveys. The LSAS survey in 2012 listed the name, address, number of beds (at end December 2012), number of current residents and number of admissions and discharges during 2012 of those centres who responded to the survey. In the 2012 survey, 78.1 per cent of nursing homes responded. The $\mathrm{NHI}$ is the representative body for private and voluntary LTRC centres in Ireland. The NHI produced a survey on the private and voluntary long-term residential centres sector in a number of years, with surveys for the years 2007, 2009-2010 and 2014 examined for this project. This survey collected information on the centre name, address and number of beds. In cases where the HIQA registry, LSAS and NHI each failed to provide information on beds, finally we used the Compliance monitoring inspection reports undertaken by HIQA, which include information on the number of residents and number of vacancies (added to provide a beds estimate) on the date of inspection. These reports were also used to check for consistencies in bed numbers within the HIQA bed registry.

The most fully-populated HIQA data relate to its 2015 bed registry data, with information on 562 residential centres included. However, information on only 480 centres was included for 2012. Using centre ID, name and address, we have included information on missing centres from 2012-2014 using the LSAS and NHI surveys, as well as the HIQA Compliance monitoring inspection reports, dropping any duplicated centres. ${ }^{23,24} \mathrm{~A}$ further 17 centres not included in the HIQA bed registry were added based upon these additional information sources. The final number of LTRC centres found to be operating at some point in the 2012-2015 period was 579 . This provided us with a four-year panel of centres across counties.

One other issue with the data concerned bed numbers per centre. In some years, bed numbers in each centre were also missing from the HIQA bed registry. We

\footnotetext{
23 A number of centres saw their name change during the period, or had different names included across data sources. Care was taken to account for name changes and therefore avoid duplication of centres in the data.

24 In a small number of cases, where a centre opened or closed in a particular year, we searched local media outlets to confirm date of opening/closure. Bed data for each centre in year of opening/closing was apportioned based upon month of opening/closure.
} 
again used a combination of LSAS and NHI surveys, alongside HIQA Compliance monitoring inspection reports to populate the beds numbers for all centres.

By taking these steps, we have developed the most comprehensive LTRC database in Ireland to date, allowing us to examine changes in supply across areas over time. The number of LTRC beds included in this report reflects the maximum number of people that a centre is registered to accommodate, and therefore provides the most accurate reflection of capacity in LTRC in Ireland.

The lack of granularity in the data prevents the examination of each type of bed separately. Therefore, LTRC supply examined in this report covers all LTRC bed categories in care for older people, but does not include information from acute hospitals, the National Rehabilitation Hospital or the disabilities sector.

We assign centres to counties and LHOs based upon their address. This allows us to estimate the total number of LTRC beds within each area for each year. To examine differences in supply across regions and over time in this chapter, we examine supply at the county level, in line with the approach used in the previous report. When carrying out the substitution analyses in Chapter 7, we include LTRC data at the LHO level in order to match LTRC beds with areas of residence in HIPE. For these analyses, we construct an LTRC bed supply per 1,000 population aged 65 years and over measure for each LHO by dividing the total beds by the population aged 65 years and over in each respective LHO and year. This is outlined in Equation 3.3:

$$
\text { LTRC per capita } \text { ly }_{l}=\frac{\operatorname{LTC} \text { beds }_{l y}}{\operatorname{Pop}(65+)_{l y}}
$$

where / represents the $\mathrm{LHO}$ and $y$ the year.

\subsubsection{Trends over time}

The first report from this project illustrated the geographic distribution of LTRC beds per 1,000 population aged 65 years and over in Ireland in 2014 using the same data sources as detailed in the previous section. Smith et al. (2019) found significant geographic variation in LTRC beds. ${ }^{25}$ In Figure 3.11 and Table 3.4, we expand upon the previous report and illustrate supply across counties over time. Counties such as Kildare, Roscommon, Tipperary North and Westmeath have consistently higher per capita LTRC beds across all years. Most counties, bar Louth and Wicklow, saw reductions in per capita supply over time, with these reductions especially large in Laois and Meath. However, there is much less volatility in bed

25 There are small differences between Smith et al. (2019) and this report as Smith et al. (2019) used data from early 2015, while this report used data from end of year, 2012-2015 inclusive. 
supply within counties over time for LTRC than for home care. This is because there were very few centre openings, expansions or closures between 2012 and 2015; changes in bed supply within counties largely reflect growth in the older population over time as opposed to changes in beds.

The Gini coefficient for the distribution of LTRC beds remains approximately 0.09 (just below the 0.1 rule of thumb of large inequality) each year examined, indicating an unequal distribution across areas, but no increase or decrease in inequalities over time is observed.

FIGURE 3.11 NUMBER OF LONG-TERM RESIDENTIAL CARE BEDS PER 1,000 POPULATION AGED 65+ BY COUNTY, 2012-2015

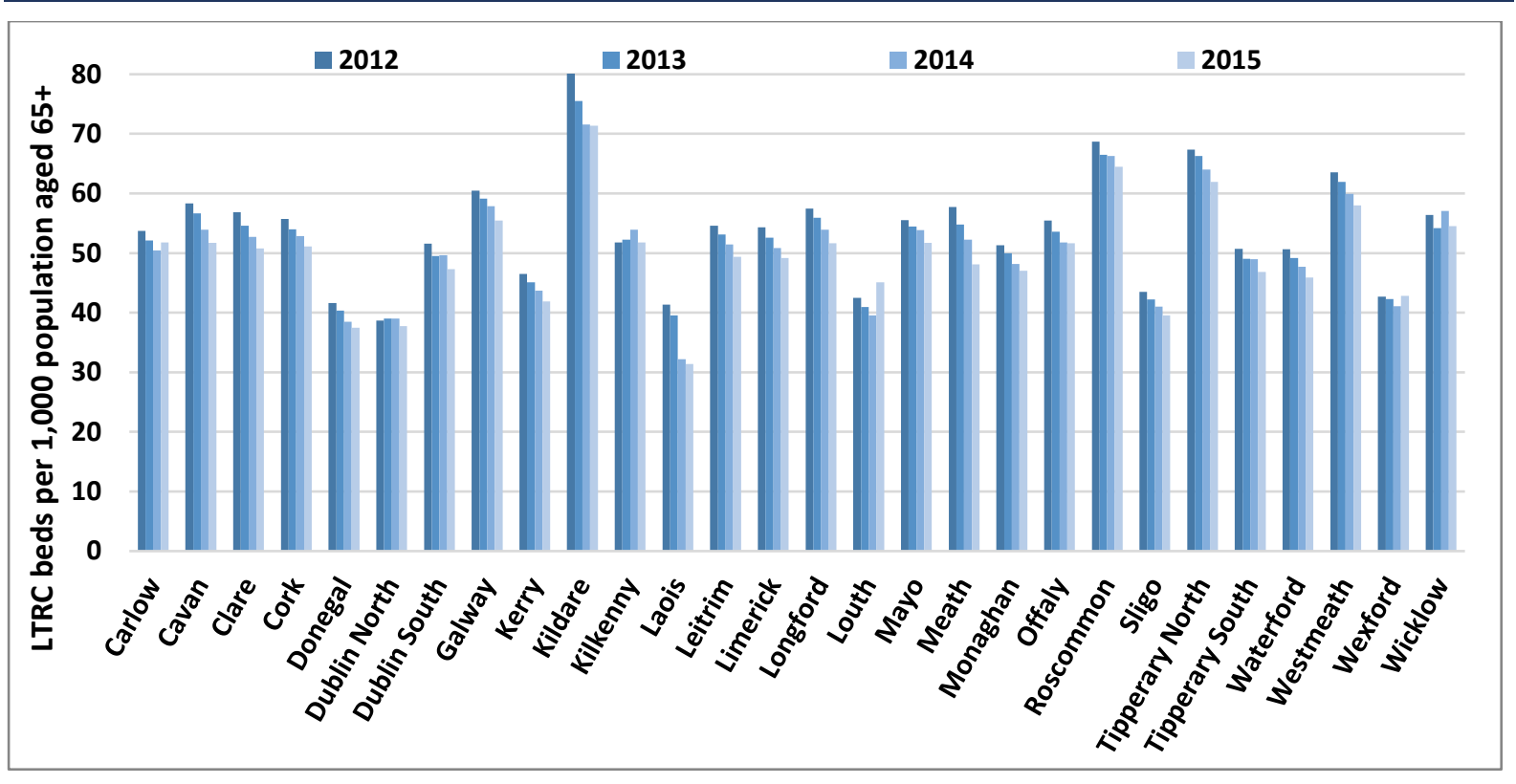


TABLE 3.4 NUMBER OF LONG-TERM RESIDENTIAL CARE BEDS PER 1,000 POPULATION AGED 65+ BY COUNTY, 2012-2015

\begin{tabular}{|c|c|c|c|c|c|}
\hline Geographic area & 2012 & 2013 & 2014 & 2015 & $\%$ change $2012-2015$ \\
\hline Carlow & 53.74 & 52.10 & 50.43 & 51.76 & -3.7 \\
\hline Cavan & 58.34 & 56.68 & 53.92 & 51.72 & -11.3 \\
\hline Clare & 56.83 & 54.60 & 52.70 & 50.75 & -10.7 \\
\hline Cork & 55.73 & 54.00 & 52.82 & 51.09 & -8.3 \\
\hline Donegal & 41.64 & 40.33 & 38.47 & 37.46 & -10.0 \\
\hline Dublin North & 38.68 & 39.03 & 38.99 & 37.73 & -2.4 \\
\hline Dublin South & 51.58 & 49.52 & 49.66 & 47.27 & -8.4 \\
\hline Galway & 60.50 & 59.10 & 57.87 & 55.43 & -8.4 \\
\hline Kerry & 46.53 & 45.09 & 43.68 & 41.88 & -10.0 \\
\hline Kildare & 80.11 & 75.53 & 71.57 & 71.37 & -10.9 \\
\hline Kilkenny & 51.77 & 52.23 & 53.93 & 51.79 & 0.0 \\
\hline Laois & 41.36 & 39.55 & 32.16 & 31.41 & -24.0 \\
\hline Leitrim & 54.60 & 53.15 & 51.44 & 49.40 & -9.5 \\
\hline Limerick & 54.32 & 52.57 & 50.83 & 49.14 & -9.5 \\
\hline Longford & 57.49 & 55.91 & 53.91 & 51.68 & -10.1 \\
\hline Louth & 42.50 & 40.92 & 39.51 & 45.08 & 6.1 \\
\hline Mayo & 55.52 & 54.47 & 53.83 & 51.72 & -6.8 \\
\hline Meath & 57.70 & 54.79 & 52.27 & 48.07 & -16.7 \\
\hline Monaghan & 51.31 & 49.96 & 48.17 & 47.03 & -8.4 \\
\hline Offaly & 55.46 & 53.55 & 51.80 & 51.62 & -6.9 \\
\hline Roscommon & 68.69 & 66.52 & 66.27 & 64.45 & -6.2 \\
\hline Sligo & 43.50 & 42.19 & 41.02 & 39.52 & -9.2 \\
\hline Tipperary North & 67.32 & 66.28 & 64.04 & 61.92 & -8.0 \\
\hline Tipperary South & 50.69 & 49.01 & 48.98 & 46.83 & -7.6 \\
\hline Waterford & 50.66 & 49.17 & 47.73 & 45.88 & -9.4 \\
\hline Westmeath & 63.56 & 61.94 & 59.95 & 58.01 & -8.7 \\
\hline Wexford & 42.68 & 42.31 & 41.10 & 42.84 & 0.4 \\
\hline Wicklow & 56.38 & 54.20 & 57.06 & 54.53 & -3.3 \\
\hline Ireland & 52.28 & 50.82 & 49.80 & 48.31 & -7.6 \\
\hline Gini Coefficient (Aged 65+) & 0.091 & 0.089 & 0.094 & 0.090 & - \\
\hline
\end{tabular}

Source: $\quad$ 2012-2015 HIQA, NHI, DoH LSAS.

Figure A.4 and Table A. 3 show the number of LTRC centres and the average size of LTRC centres (beds per centre) across counties and over time. There are differences in sizes across counties and, in general, the average size of a centre is increasing over time.

\subsection{HOME CARE AND LONG-TERM RESIDENTIAL CARE CORRELATIONS}

The previous sections separately detailed the supply of home care hours and LTRC beds across counties in Ireland. Large geographic variations in both services were observed. In Figure 3.12, using data on both home care hours and LTRC together, we plot supply of the services against one another, for the years 2012, 2013, 2014 
and 2015, to examine whether those areas with a high relative supply of one service have an accompanying high relative supply of the other.

The graphs show that there is, in general, a negative correlation in supply counties with high relative home care supply tend to have lower relative LTRC bed supply, and vice versa. The negative correlation increases over time. 


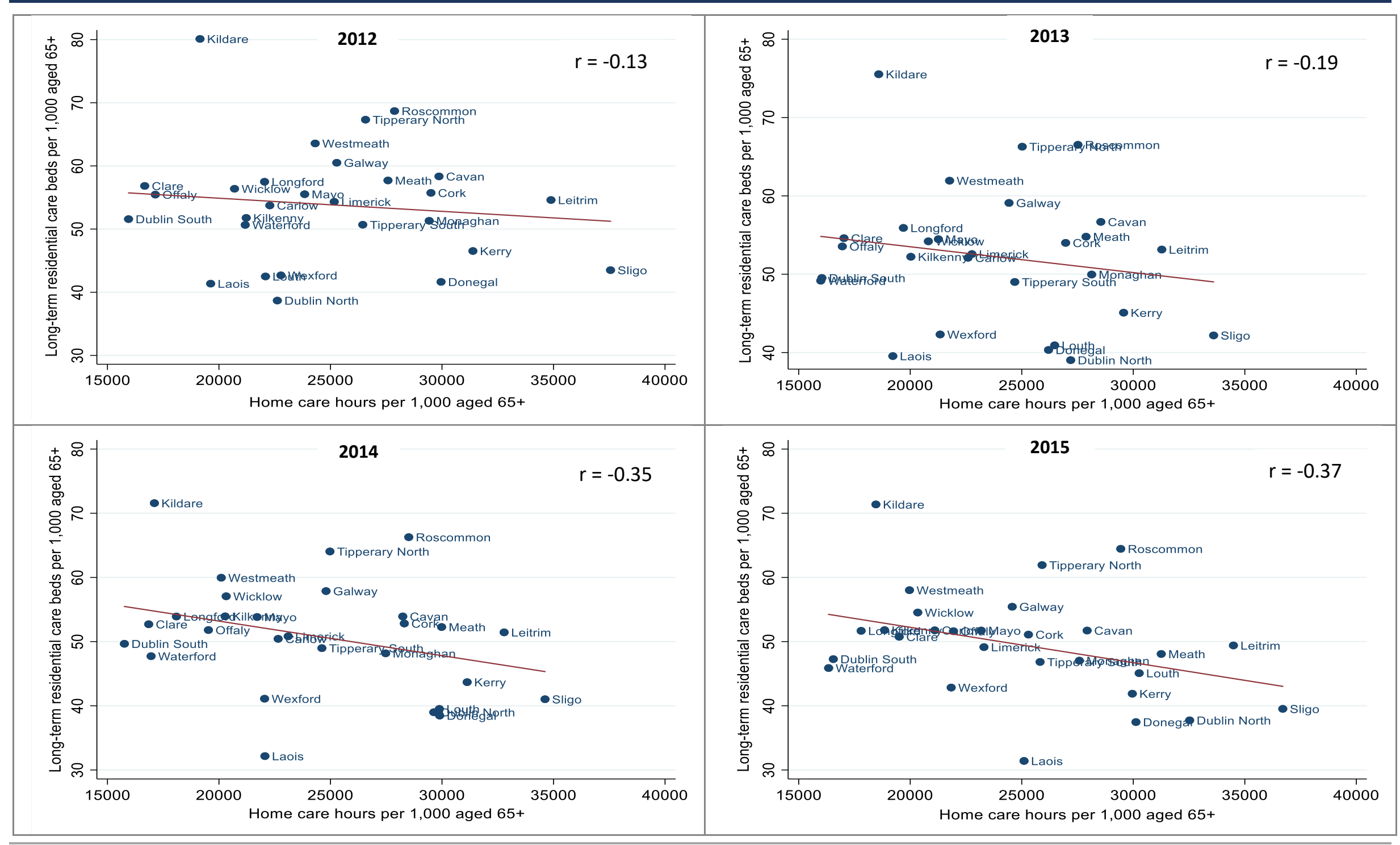




\subsection{CONCLUSION}

This chapter has profiled the supply of services by geographical area in the acute hospital, home care and LTRC sectors in Ireland. It highlights substantial changes in the acute public hospital sector in Ireland in recent years. Since the 2007-2008 period, both absolute and per capita inpatient bed capacity have changed sharply. Within an international context, this has resulted in Ireland having amongst the lowest inpatient bed capacity and the highest bed occupancy rates across the OECD. In 2015, bed occupancy rates in Ireland were 95 per cent, much higher than the internationally-recommended threshold of 85 per cent. These recent reductions in capacity and high occupancy rates underpin the projected increases in bed requirements detailed in recent government planning reports. This chapter has shown that reconfiguration of the hospital system in Ireland has impacted distance (drive time) to a Tier 1 acute hospital across areas.

This chapter has reported substantial variation in home care and LTRC bed supply across counties. In general, the variation have remained similar over time, but some areas, specifically Dublin North, have seen large increases in home care hours supply in recent years. A negative correlation was observed between home care hours supply and LTRC bed supply across counties.

Finally, this chapter has detailed the inpatient bed data, home care hours data and LTRC bed data that will be used to examine substitution of care in later chapters in this report. 


\section{CHAPTER 4}

\section{Statistical models and methodology}

\subsection{SCOPE OF THE CHAPTER}

This chapter describes the statistical methodologies and models used in chapters 5-7 to examine the impact of acute bed capacity, home care supply and long-term residential care (LTRC) bed supply respectively on emergency inpatient length of stay (LOS). Section 4.2 defines the dependent variable of interest in these analyses - inpatient LOS. Section 4.3 discusses the independent variable of interest in each analytical chapter. Section 4.4 provides details on the patients included in each analysis. Section 4.5 discusses causal inference in general, and details how this report has set out to examine substitution effects in a causal manner using the data available. Section 4.6 discusses the statistical methodologies used in chapters $5-7$, and how inpatient LOS is modelled as a dependent variable.

\subsection{DEPENDENT VARIABLE}

\subsubsection{Inpatient length of stay}

In this report, we examine the impact of acute hospital bed capacity, home care and LTRC supply on utilisation of hospital services in public hospitals in Ireland. Hospital In-Patient Enquiry (HIPE) data allow us to examine the full population of public hospital inpatients over a number of years. This provides us with a more accurate measure of hospital utilisation as compared to survey-based data that rely on recall (Bhandari and Wagner, 2006; Kjellsson et al., 2014). However, due to the lack of a unique individual health identifier $(\mathrm{IHI})$ in Ireland, it is not possible to follow patients through HIPE, or other parts of the healthcare system. Therefore, for individuals, we are unable to count the number of hospital inpatient episodes, total inpatient days per annum, readmissions from hospital or use of other hospital services such as emergency department (ED) attendances or outpatient attendances. Instead, we focus on examining inpatient LOS as the measure of hospital use.

Within HIPE, LOS refers to the time, expressed in days, between admission to and discharge from hospital (HIPE, 2009). LOS is often used as a key measure of hospital resource use in studies from Ireland (Keegan and Smith, 2013) and within the international literature (Ham et al., 2003; Martin and Smith, 1996; OECD, 2017b). A number of health systems, including Ireland, have actively sought to reduce acute care expenditure and capacity requirements by reducing inpatient LOS. Future projections of acute hospital capacity in Ireland have assumed achievement of lower inpatient LOS (Government of Ireland, 2018a; PA Consulting, 2018), by increasing primary, community and long-term care supply. Therefore, inpatient 
LOS is an appropriate measure to examine substitution of acute and non-acute services in this report. A range of studies from a number of healthcare systems have previously examined substitutability of acute and non-acute services using inpatient LOS as the dependent variable of interest, as detailed in Chapter 2.

\subsubsection{Delayed discharges}

While inpatient LOS is the main dependent variable of interest examined in the analytical chapters of this report, there is wide variation in inpatient LOS. If hospital resource use was expressed as an inpatient bed day, those with the longest LOS would use the largest proportion of bed days annually. Therefore, to free up beds and reduce overall bed day use, targeting those with the longest LOS may be most beneficial for policymakers. In this report, we also examine patients in HIPE with long LOS, many of whom are classified as delayed discharges. Both acute and nonacute supply is likely to have a greater impact on LOS for those in hospital for a longer duration, a group that is therefore of particular interest to policymakers (Gaughan et al., 2017b).

For many patients with inpatient stays of longer durations, where the majority of the stay occurs after the principal surgical and/or medical care has already been delivered, the final inpatient days could more appropriately be spent at home or in rehabilitation, convalescent care or LTRC facilities. As well as benefiting patients, this would have the effect of reducing LOS and the costs associated with it, as well as freeing up beds to improve access for elective care. However, often the lack of suitable non-acute care facilities constrains the scope for early discharge from hospital. The failure to discharge or transfer patients to more appropriate facilities results in avoidable negative consequences for patients' quality of life, and avoidable capacity and cost consequences on behalf of the hospital and health system. Longer LOS is associated with increased probability of death (Green et al., 2017), higher infection risks (Glance et al., 2011), deconditioning and poorer mobility outcomes (Fisher et al., 2010) and overall lower patient quality of life (Kossovsky et al., 2002). The longest stayers are also most likely to be older patients, for whom the negative consequences of a delayed discharge will be exacerbated. Many patients also have much greater preference for care provided close to or at home (Costa-Font et al., 2009; Fried et al., 2000).

The economic and resource consequences of unnecessarily long LOS and delayed discharges are also becoming clear. Of those countries with the highest inpatient bed occupancy rates in the OECD (Ireland, England and Canada) delayed discharges are major issues contributing to these countries experiencing bed occupancy rates exceeding 90 per cent. In 2015, 1.15 million beds days in National Health Service (NHS) England were a result of delayed discharges at a cost of over $£ 800$ million, with almost all delays amongst older patients (National Audit Office, 2016). In Canada, patients awaiting discharge from hospital (awaiting 'alternative level of 
care' (Sutherland and Crump, 2013)) account for up to 13 per cent of hospital bed days per annum (2.4 million bed days) (Canadian Institute for Health Information, 2010). In Ireland, where average bed occupancy in public hospitals is at 95 per cent, over 8,817 patients were awaiting discharge in 2017 after being medically assessed as ready for discharge, with 90 per cent of delays accounted by those aged 65 years and over (Department of Health, 2018b). This translated into 205,047 bed days lost through delayed discharges in Ireland, arguably twice as severe as figures from England. ${ }^{26}$ In each country, while the causes of delayed discharge are multifactorial, lack of long-term care provision, or waiting times for long-term care, is central. In Ireland, 15 per cent of delayed discharges were awaiting home care, while 30 per cent were awaiting some form of LTRC (Department of Health, 2018b). Between 2011 and 2015, the number of delays as a result of waiting specifically for home care increased by 172 per cent in the NHS (Edwards, 2017).

The extent of delayed discharges in Ireland is likely to affect various negative aspects of the public hospital sector in Ireland, especially in a system experiencing severe capacity constraints (Keegan et al., 2018a). Patients whose discharges are delayed reside in beds that could be used by other patients, especially those awaiting elective procedures, and this may be contributing to long elective waiting lists, long ED waiting times and waiting on 'trollies' prior to admission to an inpatient bed.

Due to data limitations, in this report we are unable to determine whether a specific patient in HIPE is characterised as a delayed discharge - that they have been medically assessed by a consultant as fit for discharge. Therefore, we use specific statistical modelling techniques (quantile regression), which allow us to model the impact of non-acute service supply on reducing LOS for those across the LOS distribution, controlling for a range of other pertinent factors. In this context, we can more easily examine LOS for those patients most likely to be in the delayed discharge category owing to their long LOS.

\subsubsection{Discharge to long-term residential care}

In Chapter 7, where we examine the impact of LTRC bed supply on inpatient LOS, we conduct some of our analyses on those who are discharged to LTRC. In this context, and as we control for home care supply as a confounding variable in Chapter 7, we also run a sub-analysis to examine whether better home care supply is associated with a lower probability of discharge from hospital to an LTRC centre.

$26 \quad 1.15$ million delayed discharge beds days in England, with a population of 54.5 million, equates to 1 per 47 population. 205,047 delayed discharge beds days in Ireland, with a population of 4.78 million, equates to 1 per 23 population. 


\subsection{INDEPENDENT VARIABLES OF INTEREST}

In this report, we examine both the 'push' and 'pull' that may impact the LOS of inpatients in public hospitals, as detailed in Chapter 2. Our main 'push' factor of interest is inpatient bed supply, while our two 'pull' factors of interest are home care hours supply and LTRC bed supply for patients aged 65 years and over. We detail these variables and how they are included in our regression analyses below.

\subsubsection{Inpatient bed supply}

Inpatient bed availability in public hospitals is the key independent variable in Chapter 5. As discussed in the previous chapter, inpatient bed data in public hospitals were provided by the Planning and Business Information Unit (BIU) in the HSE. These data provide the average monthly number of regularly maintained and staffed acute inpatient beds available for all acute public hospitals in Ireland between 2010 and 2015 (see Equation 3.1). Due to the large differences in the size of hospitals across Ireland, it is difficult to examine changes in bed supply in both an absolute and relative manner. The standardised variable treats changes in supply (as measured by standard deviations from the mean) across hospitals more appropriately. This standardised measure is the key variable of interest in Chapter 5 , where the impact changes in inpatient bed capacity on LOS is examined. However, a variant of the measure is also included in chapters $6-7$, with the standardised variable estimated for the years 2012-2015. The inclusion of inpatient bed supply as a confounding variable in chapters 6-7 allows us to examine the 'pull' factors of interest, controlling for the importance of inpatient bed supply.

\subsubsection{Home care hours supply}

The independent variable of interest in Chapter 6 is home care hours supply. Data on publicly financed home care were provided by the HSE Social Care Division. These data capture the number of home care hours provided within the home help scheme and the number of packages within the home care package (HCP) scheme across the 32 Local Health Offices (LHOs) for each month between 2012 and 2015. As discussed in Chapter 3, we merge information from both schemes to estimate an overall home care hours supply per capita variable (see Equation 3.2). This measure provides us with a home care supply measure that accounts for changes in supply over time, differences in population size across LHOs and differences in both home care supply and population within LHOs over time. In this report, we take advantage of the fact that supply of home care available differs significantly across areas and that potential home care supply available to an individual depends upon their area of residence rather than the hospital in which they receive their inpatient treatment. Therefore, there is no scope for patients to gain access to additional home care by choosing to attend a particular hospital. As noted earlier, Ireland has a large number of hospitals relative to the size of its population. Crucially (for our empirical strategy), the catchment areas of hospitals overlap in a 
number of areas (see Chapter 3). Therefore, for many hospitals, inpatients from a number of LHOs with different levels of home care supply will be included in the analyses at hospital level. This means that our statistical analyses can compare LOS for patients within the same hospital but with different supply of home care in their LHOs, controlling for a range of other pertinent patient-level information. Our analyses, therefore, can more appropriately control for unobserved hospital-level effects, which may have an impact on treatment decisions, quality of care and importantly inpatient LOS.

Between 2012 and 2015, Dublin North saw a large increase in home care provision. To examine the relationship between home care and inpatient LOS here, we use variation in home care supply at the month level. As population numbers by month in individual regions are not available, we use absolute differences in home care hours for each month in the analyses.

\subsubsection{Long-term residential care bed supply}

The independent variable of interest in Chapter 7 is LTRC bed supply. In this report, using data from a combination of the HIQA bed registry, the Department of Health's Long-stay activity statistics (LSAS) survey, Nursing Homes Ireland (NHI) surveys, and Compliance monitoring inspection reports undertaken by HIQA, we estimate the number of LTRC centres and LTRC beds in each county and LHO for the years 2012 to 2015 inclusive. We describe these data sources in detail in Chapter 3 (see Equation 3.3).

This measure provides us with an LTRC bed supply measure that accounts for changes in supply over time, differences in population size across LHOs and differences in both LTRC bed supply and population within LHOs over time. In this report, we use the supply of LTRC beds within an individual's LHO to examine the impact of LTRC supply on inpatient LOS. However, unlike publicly-provided home care, where access and supply is based upon an individual's address, individuals have greater choice of an LTRC centre. Individuals may choose a centre most suitable to them, provided there is an available bed, and this choice may not necessarily be within an individual's county or LHO. In these analyses, due to lack of data on choice of centre, we use supply in each patient's LHO as the means to measure substitution between LTRC supply and inpatient LOS.

\subsubsection{Confounding variables}

The rich HIPE dataset allows us to control for a number of confounding variables, at the patient-level and hospital-level, within our analyses. The ability to control for confounders, especially patient-level casemix, allows us to better isolate the effect of our variables of interest on LOS. 
Using information from HIPE, the statistical analyses in each of the subsequent chapters control for the following variables: day of inpatient admission; year of inpatient admission (which equals year of discharge for over 98 per cent of inpatients in HIPE); age of patient (included linearly and as a squared function); sex of patient; and marital status (married or not) of the patient.

In most analyses, we control for whether the patient stated they were covered by a medical card. Medical card status is a proxy for whether or not the patient's inpatient stay was covered by the State. As medical card eligibility is often based upon a means test, this variable is also a proxy for socioeconomic or deprivation status, as those with a medical card are more likely to come from a lower income household. Unfortunately, patient-level socioeconomic status is unavailable within HIPE. In some analyses and robustness checks, in lieu of medical card status, we also examine whether the patient was a publicly or privately treated patient. Within HIPE, a variable called 'discharge status' is captured; this 'refers to the public/private status of the patient on discharge and not to the type of bed occupied' (Healthcare Pricing Office, 2016a) and is a proxy for whether or not the patient is covered by private health insurance.

The diagnosis-related group (DRG) for each patient is also included in the regression analyses. The HPO states that 'the DRG scheme enables the disaggregation of patients into homogeneous groups, which are expected to undergo similar treatment processes and incur similar levels of resource use. The data required for DRG assignment include principal and additional diagnoses, procedures performed, age, sex, and discharge status' (Healthcare Pricing Office, 2016a). Where the analyses is examining a specific patient group, such as those who have suffered a stroke or a hip fracture, or patients with Alzheimer's disease/dementia, a DRG dummy is not included.

A weighted Charlson comorbidity score is included linearly and as a squared function for each patient in the analyses. The Charlson comorbidity index is used to predict mortality by classifying or weighting comorbid conditions such as cancer, stroke, liver disease and renal disease, with the score weighted to each comorbid condition based upon the relative risk of mortality within 12 months (Li et al., 2011). The higher the score, the 'sicker' the patient. Using the DRG and weighted Charlson comorbidity score in combination allows to more accurately compare patients of differing illness. When undertaking hospital-month level analyses in Chapter 5 , it is not straightforward to incorporate patient casemix, which arises at the individual rather than aggregate level. As a proxy for casemix in models collapsed to the hospital-month level, we include the average number of diagnoses per patient. This variable is constructed by simply counting the individual number of diagnoses listed in the HIPE record. 
Where applicable we control for admission source. In Chapter 5, we group admission sources into the following four categories: admitted from home; admitted from a nursing home/convalescent home or other long-stay accommodation; admitted via transfer from another hospital; $;{ }^{27}$ and other. ${ }^{28}$ In chapters 6 and 7, we only include those admitted from home.

Where applicable, we control for discharge destination, dependent upon the patient sample examined in each chapter. In Chapter 5, we group discharge destination into the following five categories: discharged home ${ }^{29}$ discharged to a nursing home, convalescent home, long-stay accommodation or external rehabilitation facility; died at discharge; ${ }^{30}$ transferred to another hospital; ${ }^{31}$ and other. ${ }^{32}$ In chapters 6 and 7, we exclude those who died, in line with other studies examining substitution between hospital care and home care and LTRC (Gaughan et al., 2017a; Gaughan et al., 2015).

In all analyses, a year trend is included to capture any secular trend in LOS that affected the whole sample over time. Robustness checks using time fixed effects are also undertaken, though they do not affect the results significantly. Seasonlevel fixed effects are also included to account for differences in demand of hospital care and non-acute care supply across the year (for example, winter initiatives). Month-level fixed effects are not included as in some analyses inpatient bed capacity and home care variables are included at the month level, and are therefore a linear combination of the month effects; it is inappropriate to include both measures simultaneously (Tefft, 2011).

While we control for some hospital-level characteristics, these will fail to capture unobserved differences across hospitals that may affect LOS. These unobserved differences may be quite large and may affect interpretation of results. A recent study using decomposition analyses found large unexplained differences in caesarean section rates across hospitals in Ireland after accounting for maternallevel, clinical-level and hospital-level characteristics (Brick et al., 2016). Hospital fixed effects are therefore included in all analyses to capture any systematic or unobserved hospital-level heterogeneity that may affect LOS. Furthermore, we cluster standard errors at the hospital level. As there is considerable overlap between hospitals and LHOs, there are relatively few LHOs included in these

\footnotetext{
27 Includes patients with the admission sources: 'transfer from acute hospital'; 'transfer from non-acute hospital not in HIPE hospital listing'; 'transfer from hospice not in HIPE hospital listing'; and 'transfer from psychiatric hospital/unit'. Includes patients with the admission sources: 'new born'; 'temporary place of residence'; 'prison'; and 'other'. Represents the vast majority of discharges. Includes patients with the discharge destinations: 'died with post mortem'; 'died no post mortem'. Includes patients with the discharge destinations coded by HIPE: 'transfer to hospital - emergency'; 'transfer to hospital - non emergency'; 'transfer to psychiatric hospital/unit'; 'transfer to non-acute hospital not in HIPE hospital listing - emergency'; 'transfer to non-acute hospital not in HIPE hospital listing - non emergency'; and 'hospice (not in HIPE hospital listing)'.

32 Includes patients with the discharge destinations: 'self discharge'; 'prison'; 'absconded'; 'other (e.g. foster care)'; and 'temporary place of residence (e.g. hotel)'.
} 
analyses. The non-acute supply variables are estimated at the LHO-year level; we do not include an LHO fixed effect (or a Resid fixed effect in Chapter 5) as the models become oversaturated when they are included and much of the variation in non-acute supply is accounted for by the area-level fixed effect and year trend.

\subsection{PATIENT SAMPLE}

When examining the impact of acute and non-acute care supply on inpatient LOS, health data limitations in Ireland constrain the types of patients we can include. First, we only include patients from public hospitals in Ireland as these are the patients covered by the HIPE dataset. We are unable to examine private hospital patients as no returns are made by private hospitals to HIPE or any other centralised database. Second, we only examine emergency inpatients in public hospitals. Due to the lack of information on private hospitals, it is difficult to examine elective inpatient care as many patients can substitute public hospital care with private hospital care. This is in contrast to emergency admissions where the vast majority of activity, especially complex cases, is undertaken in public hospitals. Elective procedures are also increasingly undertaken as day patient procedures as technology has improved (in public and private facilities). Therefore, it is difficult to examine changes in elective inpatient LOS over time given that the composition of the sample having an elective admission changes over time and the potential for significant bias related to omitted variables. Patients may select which public or private hospitals to receive elective care, and make their choice based upon objective or subjective quality measures, such as lower LOS. Due to the urgent nature of many emergency procedures, choice of public hospital is much less likely to be a cause of concern for emergency inpatient LOS. Furthermore, as approximately 80 per cent of inpatient bed days in acute public hospitals are emergency, rather than elective (Keegan et al., 2018b), curtailing our analysis to just emergency inpatients still results in our analyses examining the majority of inpatient care.

The lack of an IHI in Ireland reduces our ability to examine various aspects of hospital resource use, including number of hospitalisations per annum or readmission to hospitals within 30 days. The lack of an $\mathrm{IHI}$ also leads to complications when examining LOS for emergency inpatients. Many emergency inpatient discharges in HIPE have been transferred from another (smaller, less specialised) hospital; these patients may therefore have already received some of their treatment and had an inpatient admission in their previous hospital (a previous inpatient stay). For this reason, we do not include those patients where HIPE has stated they were transferred from another hospital. While the majority of emergency inpatient admissions were admitted directly from an ED, a small number were also admitted from other emergency service facilities, such as medical assessment units (MAUs). As patients admitted from an MAU may have been admitted from another hospital, or already begun their treatment during 
their MAU stay, only discharges admitted directly from an ED are included in most analyses.

During the period of study (2010-2015), as detailed in Chapter 3, there was a significant restructuring of the public hospital system in Ireland. Many hospitals were reconfigured, and in some cases hospitals lost their Tier 1 ED status. Therefore, to reduce bias caused by reconfiguration, in some analyses we curtail our patient sample to those with an inpatient stay in the 26 Tier 1 ED hospitals serving adults..$^{33}$ In addition, the three Dublin-based children's hospitals that only cater to patients aged 15 years or under are not examined here. The 26 Tier 1 ED hospitals accounted for 91 per cent of emergency discharges in HIPE between 2010 and 2015 (see Table 4.2).

Due to variations across models in the outcome variable being examined and in the data available, the sample of patients differs slightly across the analytical chapters. Chapter 5 includes inpatients, of all ages, discharged between 2010 and 2015 inclusive. The variable of interest in Chapter 5 is inpatient bed capacity and the data provided to us include maternity beds for those hospitals who provide both maternity and non-maternity care, as well as beds in wards that may be used predominantly by children. Therefore, we include emergency inpatients of all ages in our analyses (subject to the inclusion criteria set out above), though exclude patients coded as maternity. We exclude the six Dublin-based hospitals who primarily cater to maternity care and care for children aged under 15 years.

Due to the lack of data on home care and LTRC in 2010 and 2011, the analyses in chapters 6 and 7 are limited to the 2012-2015 period. As the variable of interest represents care for older people, we include only emergency inpatients aged 65 years and over. As in Chapter 5, we exclude the six Dublin-based hospitals who primarily cater to maternity care and care for children aged under 15 years.

We exclude patients whose area of residence is recorded in HIPE as 'of no fixed abode' or outside of Ireland. We also exclude a small number of patients with an LOS greater than 365 days.

\subsection{STATISTICAL MODELS}

We consider four complementary modelling frameworks to examine emergency inpatient LOS: a linear discharge-level model; linear hospital-level models; negative binomial models; and unconditional quantile regression (UQR) models. Within each model, inpatient LOS (or the natural logarithmic transformation of inpatient LOS) is included as the dependent variable. The linear and negative binomial

33 These 26 hospitals had Tier 1 ED status over the full 2010-2015 period, with one hospital briefly losing Tier 1 ED status in 2011. 
models examine the impact of differences in acute or non-acute supply on average LOS. The UQR model allows us to examine the impact that differences in non-acute supply have on LOS across the LOS distribution. This latter model therefore allows us to examine the different impact that non-acute care supply may have on groups of patients more likely to be experiencing delayed discharge.

\subsubsection{Linear regression}

We use two variants of the linear regression ordinary least squares (OLS) model in the analytical chapters, with LOS examined at the discharge level and hospitalmonth level. Across both models, we examine the impact of changes in acute and non-acute supply on average inpatient LOS.

The discharge-level models allow us to control for patient casemix. The appropriate functional form for regression analysis of LOS is an empirical question. The first model we try in each analysis is a pooled cross-sectional linear model. Due to the heavy right-tailed skewness of LOS data, and as we do not have zero counts (for example, as inpatients have an LOS of at least 1 day), we transform LOS in the linear regression models and examine LOS in its natural logarithmic form. This transformation brings the dependent variable closer to a normal distribution. An added benefit of logged LOS is that by transforming the independent variables of interest, we can interpret the coefficients as elasticities. These elasticities represent the predicted percentage change in LOS for the same percentage change in the independent variables of interest. Measuring these elasticities helps our understanding of the relationship between LOS and acute and/or non-acute care supply and aids interpretation of results across independent variables with different scales.

The second type of model used in the analyses is a hospital-level fixed effects linear model. The model collapses data on groups of individual discharges to measure the dependent and independent variables at the hospital-month level. This model is more restrictive than the cross-sectional linear model, and a smaller number of observations is used. A disadvantage of this hospital-month-level analyses is that it makes it much more difficult to control for patient casemix. Following previous work (Bloom et al., 2015; Propper and Van Reenen, 2010), we use the age and gender composition of discharges in each hospital-month period as controls for patient casemix. ${ }^{34}$ Additionally, the mean proportion of medical card discharges, mean number of diagnoses per discharge, and mean weighted Charlson score are also included to help control for patient casemix, case severity and socioeconomic status of patients. Once more, a log-linear model, with the natural logarithm of mean hospital-month LOS, is estimated with coefficients interpreted as elasticities.

34 Dummies included for: females aged 0-14 years, males aged 0-14 years, females aged 15-24 years, ... females aged $85+$ years, males aged $85+$ years. 
The benefit of the hospital-level fixed effects linear model is that it allows for many of the unobserved differences across hospitals to be stripped out. We can thereby assess the net effect of the predictors on the outcome variable.

\section{Interpretation of linear regression}

Linear model coefficients are straightforward to interpret. As our dependent variable of interest, LOS, is included in its natural logarithmic form, changes in the independent variables are associated with a percentage change in LOS.

Where the independent variables of interest are included as standardised variables, changes of one unit are interpreted in terms of their standard deviation. Therefore, coefficients can be interpreted as follows:

- A 1 unit (standard deviation) increase in inpatient beds would change LOS by $\beta * 100$ per cent.

Where the independent variables of interest are included in their natural logarithmic form, the coefficients can be interpreted as elasticities as follows:

- A 10 per cent increase in non-acute supply is associated with a $\beta * 0.1$ per cent change in LOS.

\subsubsection{Negative binomial model}

As LOS is a count variable, many studies in the literature use models designed explicitly to model count data such as the Poisson model, negative binomial model or the zero-inflated negative binomial model. As there are no zero counts with our analyses (all patients have an LOS of at least one day), more flexible models are not required to account for the issue of modelling zero counts in the analysis.

In this report, negative binomial models are preferred to more general count models such as Poisson. Previous studies have found Poisson to perform poorly in modelling the overdispersion in the distribution of LOS (the standard deviation of LOS is much greater than the mean) in comparison to the negative binomial model (Allison and Waterman, 2002; Blundell et al., 2002; Cameron and Trivedi, 2013; Hilbe, 2011). Furthermore, as we have clustered data at the hospital level, this has been shown to exacerbate overdispersion in count data (Hilbe, 2011), thereby indicating that a negative binomial would be more appropriate.

To determine whether the negative binomial was the correct model to 'fit' the LOS variable, we examined the ability to model the LOS variable accurately. Figure A.5 in the appendix plots the residuals from both negative binomial and Poisson models, to determine best fit of each model to the data. Models with lines close to 
0 indicate a better fit of the data. ${ }^{35}$ It is clear that the negative binomial model is a better fit of the LOS data than Poisson.

An additional advantage of the negative binomial model is that the coefficients can be easily compared to elasticities from the log-linear regression. The coefficient in a negative binomial model expresses the absolute change in LOS for a one unitchange in the independent variable of interest. In general in the analysis, results are very similar across OLS and negative binomial models. Therefore, for the sake of parsimony, we discuss only results from the OLS regressions in each analytical chapter, though negative binomial results are also presented in the relevant tables.

\section{Interpretation of the negative binomial model}

Coefficients within a negative binomial model care are interpreted in terms of the expected log of the count of the dependent variable, or as semi-elasticities. However, interpretation differs across functional forms of the independent variables of interest. Where the independent variables of interest are included as standardised variables, changes of 1 unit are interpreted in terms of their standard deviation. Therefore, coefficients can be interpreted as follows:

- A 1 unit (standard deviation) increase in inpatient beds would change the logs of expected LOS by $\beta$, or by $\beta * 100$ per cent.

Where the independent variables of interest are included in their natural logarithmic form, the coefficients can be interpreted as elasticities as follows:

- A 10 per cent increase in non-acute supply is associated with a $\beta * 0.1$ per cent change in LOS.

\subsubsection{Unconditional quantile regression}

It is possible that non-acute care supply affects the LOS of patients that are likely to have short LOS differently from those with long LOS (given their underlying condition and severity). To examine whether the association between non-acute care supply and LOS varies across the distribution of LOS, we estimate unconditional quantile regression (UQR) on inpatient LOS. The family of quantile regressions offer the ability to examine the impact of specific covariates across distributions of healthcare use. Therefore, they are potentially a powerful modelling technique to use when examining variables such as inpatient LOS, when the impact of covariates may differ for those who with lower healthcare resource use (inpatient bed days) use a little versus those with higher healthcare resource use.

Quantile regression analyses are commonly used across the areas of labour (Blau and Kahn, 2017; Redmond and McGuinness, 2019), energy economics (Harold et

35 Fit of data modelled using the countfit command in the statistical programme Stata. 
al., 2017) and education (Cullinan et al., 2018; Öckert et al., 2012). Within the health literature, quantile regressions are used to examine issues such as healthcare expenditure (Olsen et al., 2017). However, these models are seldom used to examine LOS, with most studies using linear or count data models. A study from the US used a 'conditional' quantile regression approach to examine the impact prospective payments have on inpatient LOS (Norton et al., 2002). This study found differing effects at the bottom and the top of the LOS distribution. A recent paper examining NHS hospitals by Longo et al. (2019) incorporated an 'unconditional' quantile regression to examine the impact hospital competition has on a range of efficiency indictors including LOS. Once more, this study found differing effects at the bottom and the top of the LOS distribution. Both these studies highlight the benefits of using UQR in the study of inpatient LOS.

It is increasingly clear that the unconditional variant of quantile regression is a more appropriate model than conditional quantile regression in the measurement of healthcare use (Borah and Basu, 2013; Longo et al., 2019). The main benefit of UQR is that it allows coefficients to be compared across the distribution of LOS. In this context, within UQR models the coefficient for those with short LOS can be compared with the coefficient for those with long LOS. Comparisons of coefficients within the UQR is possible as the technique marginalises the effect of the variable of interest over the distributions of all other independent variables in the model (Borah and Basu, 2013). This is not possible within conditional quantile regressions and this inability to accurately compare coefficients increases as the number of covariates included increase. Therefore, as we control for a large number of independent variables (for example, DRG dummies), the benefits of using UQRs over more traditional quantile regression are large in these analyses.

Following Firpo et al. (2009) and Longo et al. (2019), we estimate whether nonacute care supply has different effects across unconditional quantiles of the LOS distribution using a two-stage approach. First, rather than regressing LOS on our covariates, we estimate a recentered influence function (RIF). Influence functions are commonly used within statistics; ${ }^{36}$ they test the sensitivity of an estimator to the removal of an observation (patient) with different characteristics and at different parts of the outcome distribution. Here, the influence function is recentred (to 0 ) to allow for comparability of results across LOS quantiles (Equation 4.1):

$$
R I F\left(\operatorname{LOS}_{i} ; q_{\tau}\right)=q_{\tau}+\frac{\tau-1\left[\operatorname{LOS}_{i} \leq q_{\tau}\right]}{f_{L O S}\left(q_{\tau}\right)}
$$


Here, RIF captures where patients are in the LOS quantile. $q_{\tau}$ is the $\tau^{\text {th }}$ quantile of LOS, $\left[\operatorname{LOS}_{i} \leq q_{\tau}\right]$ is included only when the a patient's LOS is less than or equal to quantile $q_{\tau}$, with $f_{L O S}\left(q_{\tau}\right)$ representing the density function within each $q_{\tau}$. In the second step, the estimated RIF is regressed on the same independent variables as the linear and negative binomial models above (Equation 4.2):

$$
\operatorname{RIF}\left(L O S_{i} ; q_{\tau}\right)=\alpha_{\tau} X_{i}+\beta_{\tau} N A S_{l y}+\varepsilon_{i}
$$

Using this approach, the other independent variables (denoted by the vector $X_{i}$ ) are controlled for, and the $\beta$ coefficient on our non-acute supply (NAS) variable of interest can be interpreted in a similar way as coefficients from a linear or negative binomial.

\section{Interpretation of the unconditional quantile regression model}

Coefficients within a UQR should be interpreted in a similar way to those in a linear regression. As our independent variables of interest are included in their natural logarithmic form, the coefficients can be interpreted as follows.

- A 10 per cent increase in non-acute supply is associated with a $\beta *[\log (1.1)]$ change in $\mathrm{Y}$ for each quantile of $\mathrm{Y}$.

- In chapters 6 and 7, we estimate coefficients for the 20th, 30th, 40th, 50th, 60th, 70th, 80, 90th and 95th unconditional quantiles. To aid interpretation of results, LOS for each unconditional quantile is provided. We also discuss the elasticities in our results.

\subsubsection{Standard errors}

While the analyses are undertaken at the discharge level, patients' LOS is affected by a host of non-patient-level characteristics, such as the consultant or hospital decision to discharge them. Therefore, within these analyses, we allow for clustering of standard errors at the level of the hospital throughout. As patients are grouped within hospitals, standard errors across patients within hospitals are therefore correlated. Failure to account for within-hospital correlation will lead to spuriously small errors. This failure to account for within-group (hospital) correlation has been shown to result in incorrect statistical significance as a result of these spuriously small standard errors (Rokicki et al., 2018).

In line with asymptotic assumptions (related to the law of large numbers), a larger number of observations in a model results in more accurate, and smaller, standard errors. However, clustering standard errors can be problematic. As standard errors are estimated at the hospital (cluster) level rather than at the patient level, the asymptotic assumptions often relied upon when estimating standard errors in general may not hold when the number of clusters is small. There is evidence that a small number of clusters (fewer than 50 ) may be insufficient to estimate accurate standard errors (Cameron and Miller, 2015). In this instance, the standard errors 
may be larger than they would have otherwise been if the number of hospitals (clusters) were large. In these analyses, while we have a large number of patients included from HIPE, in many analyses we are including 26 hospitals. Therefore, while we cluster at the hospital level, standard errors estimated may be impacted by the small number of clusters. The problem relating to clustering has been addressed using different techniques in other studies of the Irish hospital sector. For example, Walsh et al. (2019) used bootstrapping methods. In general, as a result of the small number of hospitals in Ireland, the issue surrounding the most appropriate clustering will continue to affect future analyses of hospital care in Ireland.

\subsubsection{Statistical programme}

All statistical analyses were undertaken using Stata version 15.1. 



\section{CHAPTER 5}

\section{Public hospital inpatient length of stay and inpatient bed supply}

\subsection{SCOPE OF THE CHAPTER}

In this chapter, we examine the relationship between inpatient length of stay (LOS) and inpatient bed capacity in public hospitals in Ireland between 2010 and 2015. Section 5.2 details the main aims of this chapter and the questions being considered. Section 5.3 provides the institutional background to the analysis and discusses how the analyses attempts to estimate causal effects. Section 5.4 presents the findings and Section 5.5 concludes.

\subsection{QUESTION}

In this analysis, we examine whether changes in inpatient bed capacity in public hospitals in Ireland affect average inpatient LOS for emergency inpatients.

As discussed in Chapter 4, we include a specific sample of emergency inpatient hospital discharges recorded in the Hospital In-Patient Enquiry (HIPE) dataset between 2010 and 2015. We exclude all maternity and children's hospitals in the analysis. To reduce bias caused by smaller hospitals who do not provide a large amount of emergency inpatient care, we remove hospital months with fewer than 100 emergency inpatient discharges. We separately run analyses on the 26 large acute public hospitals with a Tier 1 emergency department (ED). In some analyses, readmissions are also excluded for comparability across hospitals and time.

In the analyses, we aim to estimate casual effects using exogenous shocks in bed capacity across hospitals over time. We argue that changes in bed capacity that occurred in Ireland between 2010 and 2015 were less a consequence of demand for inpatient care or a restructuring of the health system to non-acute care, but rather a result of financial pressures caused by the economic recession. We also show that changes to bed supply did not occur at the same time for each hospital, which allows us to examine variations in relative supply across hospitals at a point in time.

We estimate linear regression and negative binomial models. The independent variable of interest is standardised inpatient bed supply, and we control for a range of other confounders including: day of admission; year of admission; admission source; discharge destination; age and age squared; weighted Charlson comorbidity score (linear and squared); sex; marital status; specific diagnosisrelated group (DRG); and medical card status. Finally, to control for unobserved hospital-level factors that may affect results, we include hospital fixed effects. Due 
to the considerable overlap between Local Health Office (LHO) and hospital, we do not include LHO-level fixed effects.

\subsection{BACKGROUND}

As detailed in Chapter 2, there have been significant reductions in inpatient LOS over time across health systems generally. Studies that have examined the impact of hospital bed supply, or a proxy of bed supply such as occupancy rates, have provided ambiguous results. A 16 per cent reduction in hospital beds in Denmark between 2007 and 2014 reduced inpatient LOS from 3.9 days to 3.1 days (Christiansen and Vrangbæk, 2017), though bed reductions were accompanied by a large increase in doctors and nurses, as well as significant investment in nonacute care (Christiansen and Vrangbæk, 2017). While the Danish reconfiguration lowered LOS, other consequences of the reforms were bed shortages, high bed occupancy rates and increased hospital and 30-day mortality (Madsen et al., 2014). Where bed reductions are accompanied by high occupancy rates, negative patient outcomes will occur (Bagust et al., 1999; Boden et al., 2016; Eriksson et al., 2017; Schilling et al., 2010). Therefore, it is important to identify whether reductions in LOS are a result of cuts to acute bed capacity.

As detailed in Chapter 3, Ireland saw large reductions in bed capacity during the recession and now has one of the lowest inpatient beds per capita, and the highest occupancy rates in the OECD (OECD, 2017a). Many European countries have experienced cuts to public healthcare budgets in recent years (Mladovsky et al., 2012) with countries affected by the crisis, such as Ireland, having enacted severe cuts in public healthcare expenditure (Burke et al., 2014; Keegan et al., 2018a; Nolan et al., 2014; Wren et al., 2017). ${ }^{37}$ Studies that have examined the responses of healthcare systems to changes in resources (for example, due to a funding shock) hypothesise that hospitals may respond in three specific ways: by increasing occupancy rates; by reducing LOS; and/or by decreasing the number of admissions (Kroneman and Siegers, 2004). Greater emphasis on substituting care from the hospital to the primary/community care sector may also help reduce costs (Lehto et al., 2015). However, the ability for substitution is severely curtailed in circumstances of dramatic funding shocks, as non-acute services also tend to experience reductions in funding.

Ireland was severely impacted by the Great Recession and between 2010 and 2013 severe cuts to frontline services in both the acute and non-acute sectors were made, along with closure of inpatient beds. The reduction in inpatient bed supply was a result of explicitly removing inpatient beds from use, converting them to day

37 There is evidence that initially many EU countries enacted a counter-cyclical policy by expanding healthcare expenditure in response to recession (Keegan et al., 2013) 
patient beds and reducing staff numbers. In this study, we take advantage of changes in bed capacity across hospitals over time during the 2010-2015 period.

\subsubsection{Causal inference}

Identifying causal effects tends to be difficult to do in the absence of a clear natural experiment. However, we argue that changes in bed supply between 2010 and 2015 were primarily a result of exogenous shocks to the health system (economic recession and subsequent improvement), and were unrelated to any reduced demand for inpatient care from patients. We argue that as the economic recession also affected non-acute care provision, the reductions in bed capacity were not accompanied by major increases in the provision of non-acute care to allow for planned substitution away from hospital care.

The standardised bed measure we include (outlined in Chapter 4) allows us to compare hospitals of different size. Figure 5.1 presents the standardised bed supply across hospitals over the 2010-2015 period. As we show in Figure 5.1, the fluctuations in bed supply differed across hospitals, with some hospitals seeing reductions (increases) at earlier (later) points in time than others. This means that we are not simply capturing a time effect, and that there was considerable variation in supply across hospitals. While the national average (thick black line) saw a reduction to December 2012 followed by an increase thereafter, not all hospitals followed the same trajectory. Furthermore, changes in capacity differed across hospitals, with some hospitals seeing much greater volatility in supply than others; in other words, variation is observed across hospitals. Therefore, within our analyses, we are comparing hospitals, at the same point in time, with different (relative) bed supply.

FIGURE 5.1 STANDARDISED INPATIENT BED SUPPLY HOSPITAL, 2010-2015

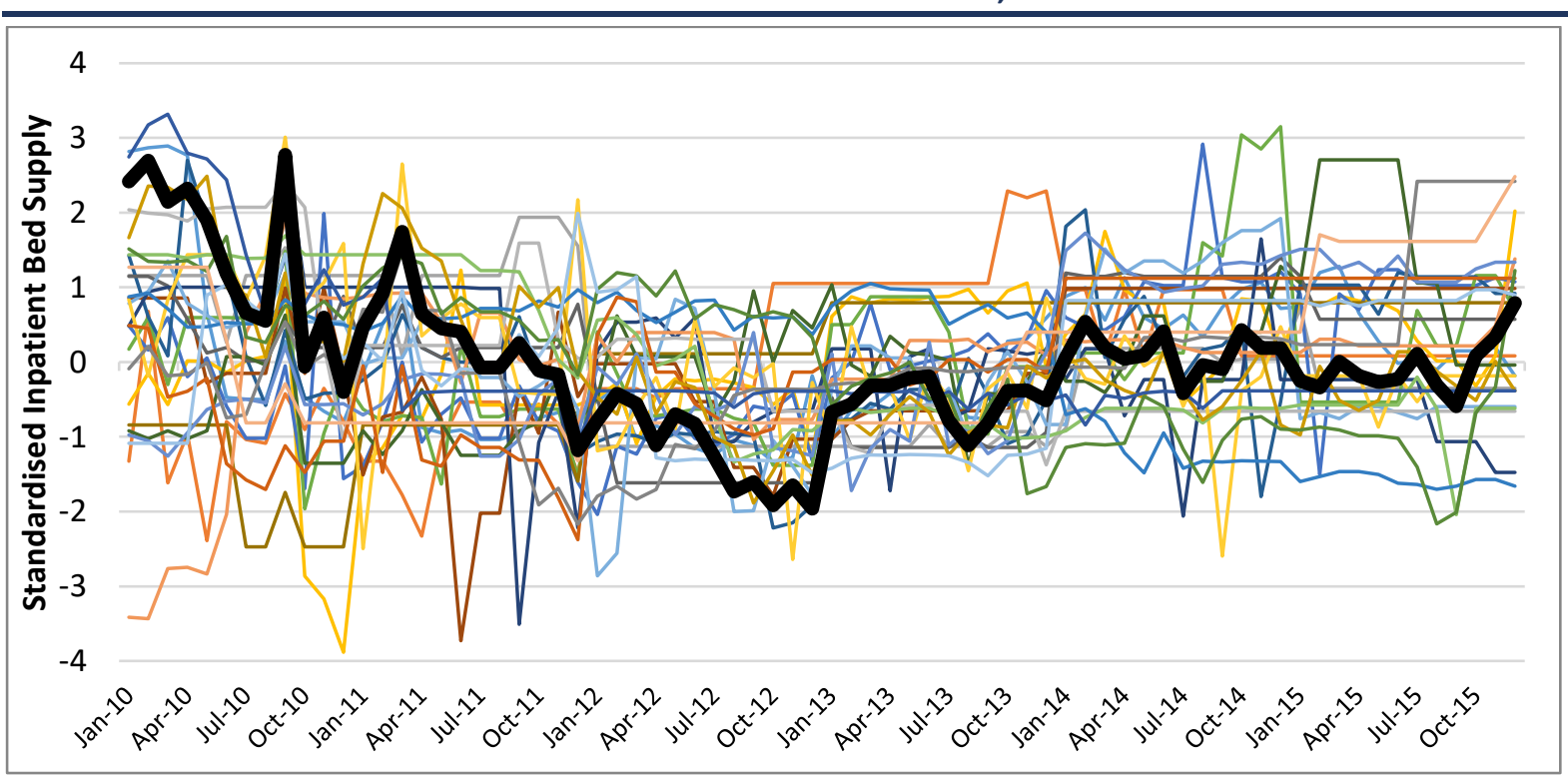


The argument that bed capacity reductions were a result of budget restraints rather than improvements in LOS efficiency has also been borne out from recognition by policymakers of the need for considerable investment in acute bed capacity (Keegan et al., 2018a). A recent government-commissioned healthcare capacity review estimated an additional 50 per cent inpatient beds would be required by 2031 (Department of Health, 2018a), partly as a result of current and previous deficits. Waiting lists for elective care are amongst the highest in developed countries (Siciliani et al., 2014), and an Independent Expert Review has also found that significant issues exist with delayed discharges, with a large proportion of delays caused by inadequate non-acute care (Department of Health, $2018 \mathrm{~b})$. Each of these sources is supportive of the view that the changes in inpatient bed supply in recent years were a consequence of exogenous financial shocks, rather than a reduction in demand or better access to non-acute care.

\subsection{RESULTS}

\subsubsection{Descriptive statistics}

Table 5.1 presents descriptive statistics of the discharge sample used in this chapter. Overall, there were 2.237 million emergency inpatient discharges between 2010 and 2015, with over 90 per cent of these discharges taking place within Tier 1 ED hospitals. Average LOS over this period was 6.42 days. The average age of the sample was 49.64 years, 58.6 per cent of discharges had a medical card and discharges had an average of 3.93 diagnoses. Admissions directly from an ED accounted for 73 per cent of discharges, while 85.7 per cent were discharged home, and 2.6 per cent died in hospital. 
TABLE 5.1 DESCRIPTIVE STATISTICS OF EMERGENCY INPATIENT DISCHARGES, 2010-2015

\begin{tabular}{|c|c|c|}
\hline & Number of discharges & \\
\hline Number of emergency inpatient discharges & $2,237,026$ & \\
\hline \multicolumn{3}{|l|}{ Year of discharge } \\
\hline 2010 & 339,994 & \\
\hline 2011 & 343,294 & \\
\hline 2012 & 375,414 & \\
\hline 2013 & 383,411 & \\
\hline 2014 & 394,963 & \\
\hline \multirow[t]{2}{*}{2015} & 399,950 & \\
\hline & Mean & SD \\
\hline Length of stay & 6.42 & 15.56 \\
\hline Tier 1 ED hospital discharges & 0.91 & 0.29 \\
\hline Age & 49.64 & 27.60 \\
\hline Medical card & 0.586 & - \\
\hline Number of diagnoses & 3.93 & 3.19 \\
\hline Weighted Charlson score & 0.78 & 1.63 \\
\hline \multicolumn{3}{|l|}{ Mode of emergency admission } \\
\hline Emergency department & 0.732 & - \\
\hline AMAU - admitted as in-patient & 0.068 & - \\
\hline Other & 0.102 & - \\
\hline AMAU only & 0.098 & - \\
\hline Readmission & 0.012 & 0.109 \\
\hline Emergency admissions per hospital per month & 1,174 & 513 \\
\hline \multicolumn{3}{|l|}{ Discharge destination } \\
\hline Home & 0.852 & - \\
\hline Long stay & 0.054 & - \\
\hline Died & 0.026 & - \\
\hline Transfer & 0.055 & - \\
\hline Other & 0.013 & - \\
\hline \multicolumn{3}{|l|}{ Admission day } \\
\hline Sunday & 0.096 & - \\
\hline Monday & 0.156 & - \\
\hline Tuesday & 0.166 & - \\
\hline Wednesday & 0.161 & - \\
\hline Thursday & 0.157 & - \\
\hline Friday & 0.157 & - \\
\hline Saturday & 0.107 & - \\
\hline \multicolumn{3}{|l|}{ Marital status } \\
\hline Single/widowed/separated/other & 0.626 & - \\
\hline Married & 0.374 & - \\
\hline
\end{tabular}

\subsubsection{Length of stay}

Figure 5.2 illustrates the average LOS for emergency inpatient discharges in all public hospitals and separately in Tier 1 ED hospitals, between 2010 and 2015. For all public hospitals, the estimates include all emergency admissions including those from MAUs, other non-ED units and readmissions. For the Tier 1 ED hospitals, only admissions directly from the ED are included. A U-shape is observed in the average 
LOS over time, with average LOS reducing between 2010 and 2012, by 6 per cent in the overall sample and 6.2 per cent in the Tier 1 ED sample respectively. However, between 2012 and 2015 an increase was observed. Overall, average LOS was still lower in 2015 than at the beginning of the period. There is evidence that the LOS increase reported in 2015 was not an anomaly, with average inpatient LOS (for those who stay overnight) in 2017 reported by HIPE to be 6.9 days. ${ }^{38}$

FIGURE 5.2 AVERAGE LENGTH OF STAY FOR EMERGENCY INPATIENT DISCHARGES, 2010-2015

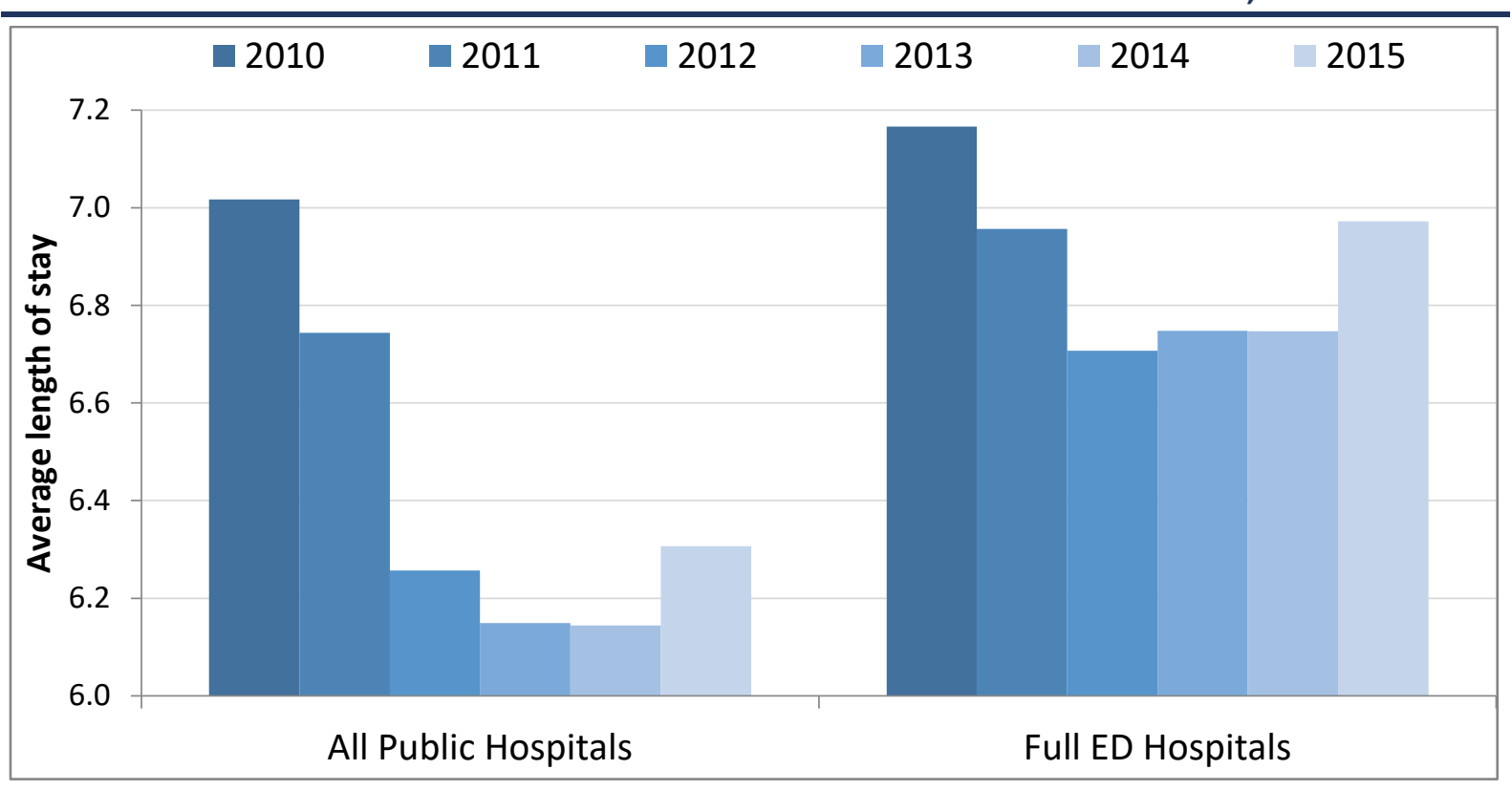

Source: LOS in Tier 1 ED hospitals include those admitted from the ED and exclude readmissions.

\subsubsection{Regression results}

Table 5.2 presents the determinants of LOS from the pooled linear regression and pooled negative binomial regression models (discharge-level models). Results show a positive and statistically significant relationship between inpatient bed supply and average LOS in all models. To provide some interpretation for these results, in the Tier 1 ED hospital sample (Column III) a 1 standard deviation (SD) reduction in inpatient bed availability implies a drop in average LOS of 1 per cent. In Tier 1 ED hospitals, the bed availability between 2010 and 2012 fell by 2.6 SDs (642 beds), implying a drop in average LOS of 2.7 per cent. This can be compared with the 6.4 per cent drop in LOS actually observed between 2010 and 2012 . Results from all public hospitals imply a drop in average LOS of 6.5 per cent, compared to the 10.8 per cent drop actually observed between 2010 and 2012 .

Other interesting results (from column III) are that patients with a medical card had 5.5 per cent longer LOS, even after controlling for confounders such as age, number

38 See http://www.hpo.ie/latest_hipe_nprs_reports/HIPE_2017/HIPE_Report_2017.pdf. 
of diagnoses and area of residence. Patients who were discharged to long-stay units (nursing homes) had much longer LOS. ${ }^{39}$

TABLE 5.2 DETERMINANTS OF LENGTH OF STAY FOR EMERGENCY INPATIENT DISCHARGES (DISCHARGE-LEVEL MODEL), 2010-2015

\begin{tabular}{|c|c|c|c|c|c|c|}
\hline & \multicolumn{3}{|c|}{$\begin{array}{l}\text { Ordinary least squares } \\
\text { (LN LOS) }\end{array}$} & \multicolumn{3}{|c|}{$\begin{array}{l}\text { Negative binomial } \\
\text { (LOS) }\end{array}$} \\
\hline & (I) & (II) & (III) & (IV) & (V) & (VI) \\
\hline $\begin{array}{l}\text { Inpatient bed } \\
\text { capacity } \\
\text { (Standardised) }\end{array}$ & $0.018 * * *$ & $0.010 * * *$ & $0.010 * * *$ & $0.018 * * *$ & $0.011 * * *$ & $0.011 * * *$ \\
\hline Medical card & $0.059 * *$ & $0.059 * *$ & $0.055^{* *}$ & $0.079 * * *$ & $0.076 * * *$ & $0.072 * * *$ \\
\hline \multicolumn{7}{|l|}{$\begin{array}{l}\text { Discharge } \\
\text { destination }\end{array}$} \\
\hline \multicolumn{7}{|l|}{ Home (Ref.) } \\
\hline Long stay & $0.766 * * *$ & $0.768 * * *$ & $0.383 * * *$ & $0.885 * * *$ & $0.827 * * *$ & $0.834 * * *$ \\
\hline Died & $0.116 * * *$ & $0.047 * * *$ & -0.003 & $0.400 * * *$ & $0.362 * * *$ & $-0.329 * * *$ \\
\hline Transfer & $0.082 * *$ & 0.035 & $0.110 * * *$ & $0.270 * * *$ & $0.233 * * *$ & $0.297 * * *$ \\
\hline Other & $-0.189 * * *$ & $-0.211 * * *$ & $-0.203 * * *$ & -0.083 & -0.078 & -0.086 \\
\hline Tier 1 ED only & No & No & Yes & No & No & Yes \\
\hline Readmissions & Yes & No & No & Yes & No & No \\
\hline Clusters & 38 & 38 & 26 & 38 & 38 & 26 \\
\hline Observations & $2,216,733$ & $1,975,782$ & $1,484,253$ & $2,216,733$ & $1,975,782$ & $1,484,253$ \\
\hline R squared & 0.431 & 0.428 & 0.437 & - & - & - \\
\hline
\end{tabular}

Notes: All models control for age, age squared, sex, weighted Charlson comorbidity index (linear and squared), marital status, day of admission, year of discharge, admission source, discharge destination, DRG, hospital fixed effects, season fixed effects and linear time trend.

Standard errors are clustered at the level of the hospital.

Hospital/month periods with less than 100 total emergency discharges are excluded.

$* \mathrm{p}<0.01,{ }^{* *} \mathrm{p}<0.05, * * * \mathrm{p}<0.01$

Table 5.3 presents the determinants of LOS from the hospital-month level linear regression model. Results once more show a positive and statistically significant relationship between inpatient bed supply and average LOS in all models. Overall, the results are similar to those shown in the previous table for both samples, with the model predicting a 2.7 per cent drop in LOS between 2010 and 2012 in the Tier $1 \mathrm{ED}$ hospital sample (column III), compared to the 6.4 per cent drop observed.

Table 5.3 also highlights that hospitals with a greater number of medical card admissions and sicker patients (as measured by the mean weighted Charlson score per discharge) have longer LOS.

39 This may in part reflect waiting lists and capacity constraints in the long-stay sector during this time period, and is examined in greater detail in Chapter 7. 
TABLE 5.3 DETERMINANTS OF LENGTH OF STAY FOR EMERGENCY DISCHARGES (HOSPITAL-LEVEL MODEL), 2010-2015

\begin{tabular}{l|c|c|c|}
\hline & \multicolumn{3}{|c|}{$\begin{array}{c}\text { Ordinary least squares } \\
\text { (Ln LOS) }\end{array}$} \\
\hline & (I) & (II) & (III) \\
\hline $\begin{array}{l}\text { Inpatient bed capacity } \\
\text { (standardised) }\end{array}$ & $0.019 * * *$ & $0.013^{* * *}$ & $0.013^{* *}$ \\
\hline Mean medical card & 0.042 & $-0.168^{* *}$ & $-0.148^{* * *}$ \\
\hline Mean weighted Charlson & $0.379^{* * *}$ & $0.238^{* * *}$ & $0.234^{* * *}$ \\
\hline Tier 1 ED only & No & No & Yes \\
\hline Readmissions & Yes & No & No \\
\hline Clusters & 38 & 38 & 26 \\
\hline Observations & 2,505 & 2,480 & 1,867 \\
\hline
\end{tabular}

Notes: All models control for the age/gender composition of discharges, total emergency cases by hospital/month, hospital fixed effects and linear time trend.

Standard errors are clustered at the level of the hospital.

Hospital/month periods with less than 100 total emergency discharges are excluded.

Discharges with the longest 1 per cent of LOS are excluded: $>60$ days.

${ }^{*} \mathrm{p}<0.01,{ }^{* *} \mathrm{p}<0.05,{ }^{* * *} \mathrm{p}<0.01$

Inpatient bed supply, acting as a 'push' factor, may have differing effects on LOS across patients with different characteristics. Therefore, in Figure 5.3, we interact inpatient bed supply with sex, marital status, private discharge status and weighted Charlson comorbidity score to test whether reductions in inpatient supply may reduce LOS at a greater rate for females (versus males), married patients, private patients or sicker patients with more comorbidities. ${ }^{40}$ Results are based upon the linear regression model from column III in Table 5.2. Overall, we find that there is little heterogeneity in the effect of inpatient bed supply on inpatient LOS in the groups examined.

40 Regarding 'private discharge status', public/private discharge status is included in the regression instead of medical card status to estimate findings for this figure. 


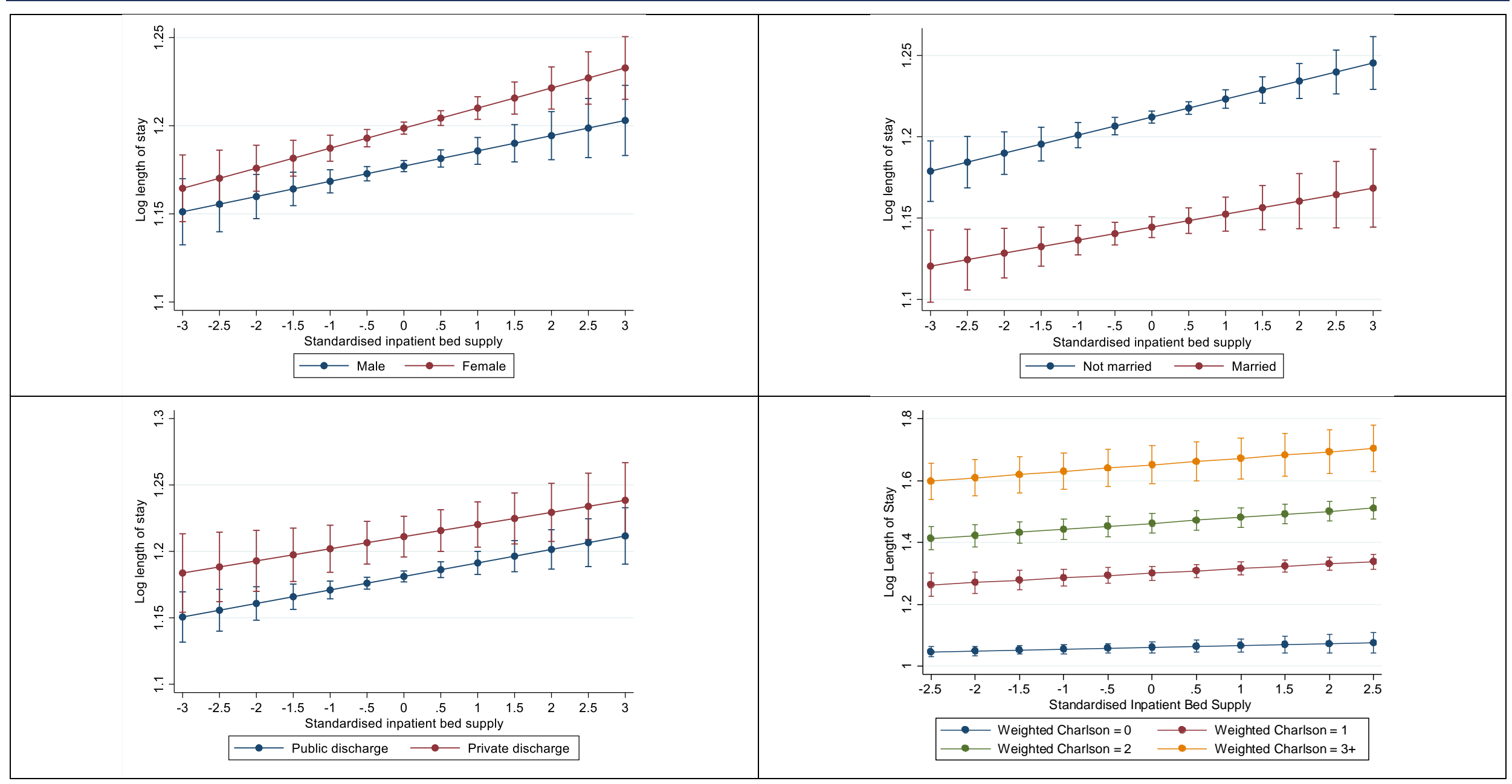

Ordinary least squares regressions based on Table 5.2, column III. Covariates in models: year, married, medical card (or public/private status), weighted Charlson score, hospital FEs, DRG, hospitallevel standardised bed capacity, age and age squared, season.

Sample: patients admitted from home through an ED, not transferred to another hospital at discharge, alive at discharge.

Clustered standard errors at hospital level. 


\subsubsection{Sensitivity analyses}

A number of sensitivity analyses were undertaken to determine if the relationship between inpatient bed availability and LOS was more pronounced in some groups. The interaction coefficients between bed supply and whether the patient had an operation/surgery or not as part of their emergency inpatient stay, discharge destination and hospital size were found to be insignificant. The relationship between bed supply and LOS was found to be larger amongst older patients.

\subsection{CONCLUSIONS}

This chapter finds that the changes in inpatient LOS in Ireland between 2010 and 2015 were closely related to changes in bed capacity that occurred during those years. Descriptively, there was a clear U-shaped pattern in average LOS in these years, corresponding to a similar pattern in inpatient bed supply. In each of the regression analyses, controlling for a range of pertinent patient-level characteristics and discharge casemix, we find a positive, statistically significant relationship between LOS and bed capacity; a higher level of bed capacity is associated with longer LOS.

In order to interpret the coefficients and help illustrate the magnitude of the effects, we use the changes in average LOS between 2010 and 2012 as points of reference. These years also equate to the period where the most severe public healthcare expenditure cuts were experienced. Overall, the analysis predicts that approximately 40-60 per cent of the reduction in LOS for emergency inpatients observed between 2010 and 2012 may have been a result of bed capacity reductions experienced in those years. 


\section{CHAPTER 6}

\section{Does formal home care reduce public hospital inpatient length of stay and delayed discharges?}

\subsection{SCOPE OF THE CHAPTER}

In this chapter, we examine the relationship between inpatient length of stay (LOS) and public home care supply in Ireland for older people between 2012 and 2015. Section 6.2 details the question examined and the patients included in the study. Section 6.3 summarises the background to how we have tried to estimate casual effects in this study. Section 6.4 presents the findings and Section 6.5 concludes.

\subsection{QUESTION}

In this analysis, we aim to estimate the impact of the supply of public home care on emergency inpatient LOS in public hospitals in Ireland amongst patients aged 65 years and older. We explicitly examine the impact that home care has on those with long LOS, many of whom are likely to be classified as delayed discharges.

We use variations in home care supply across areas and over time as a means to identify causal effects. Furthermore, we exploit the fact that availability of public home care is based upon the patient's address (Local Health Office (LHO) area) rather than the hospital they attend. This allows us to compare patients attending the same hospital but with differing access to home care, which helps us to better control for unobserved hospital-level effects that may affect a patient's LOS. Home care may affect the use of hospital services by: a) reducing hospital use, in particular inpatient admissions; and b) reducing LOS of an inpatient hospital admission. We focus on the latter channel due to data constraints, because the lack of an individual health identifier (IHI) in Ireland prevents us following individuals across separate inpatient admissions.

As discussed in Chapter 4, in this chapter we include emergency inpatient hospital discharges, admitted through an emergency department (ED) in the Hospital InPatient Enquiry (HIPE) dataset between 2012 and 2015. To reduce bias caused by smaller hospitals who do not provide a large amount of emergency inpatient care to older people, we curtail our analyses to the 26 large acute public hospitals with a Tier 1 ED. Readmissions were also excluded for comparability across hospitals and time. We only include patients aged 65 years and older at discharge as this is the group targeted by home care in Ireland. ${ }^{41}$ We only include patients admitted from home, as home care may be most applicable to them. In line with previous 
analyses of LOS and long-term care supply (Gaughan et al., 2015), we exclude patients who died in hospital. We further exclude patients who are transferred to another hospital or setting. These inclusion criteria focus our analysis on a set of patients that has the potential to use home care when discharged, and it allows for consistency across hospitals, areas and time. The sample includes 304,005 discharges of patients aged 65 years and over between 2012 and 2015.

We undertake sub-analyses in this chapter to examine the robustness of results across different patient sub-populations. First, owing to the large increases in home care seen in Dublin North between 2012 and 2015, we undertake analyses on patients with a Dublin North residence ID who were discharged from hospitals within the Dublin North catchment area. Second, we examine two specific patients groups seen in the literature as most amenable to home care supply: stroke and hip fracture patients (Gaughan et al., 2015; Huckfeldt et al., 2014). Patients with a primary or secondary diagnosis of stroke were identified using ICD-10 classification codes $160,161,163$ and 164, resulting in 8,671 stroke discharges. Patients with a primary diagnosis of hip fracture patients were identified using ICD-10 codes S7200, S7201 and S7220, resulting in 6,136 hip fracture discharges. Third, we examine those aged 75 years and over and 85 years and over separately, because while public home care is targeted at people 65 years and over in Ireland, in practice usage rates of home care are much higher in the oldest age groups (Wren et al., 2017). Heterogeneity in the effect of home care on LOS is examined across specific demographic groups, including gender, marital status, public/private status and comorbidity.

We estimate linear regression and negative binomial regression models to examine the effect of home care supply on average LOS. To examine whether the association between home care provision and LOS varies across the distribution of LOS, we estimate unconditional quantile regressions (UQRs). We control for a range of other confounders including: day of admission; year of admission; admission source; discharge destination; age and age squared; weighted Charlson comorbidity score (linear and squared); sex; marital status; specific diagnosisrelated group (DRG); and medical card status. Finally, to control for unobserved hospital-level factors that may affect results, we include hospital fixed effects. Due to the considerable overlap between LHO and hospital, we do not include LHOlevel fixed effects. A standardised measure of the number of inpatient beds at the hospital-month level is also included to account for bed capacity changes in each hospital over the period.

\subsection{BACKGROUND}

As discussed in Chapter 3, there is a paucity of evidence internationally on substitution between acute care and care for older people, with no quantitative 
evidence yet available for Ireland. Evidence from England shows that while social care supply and expenditure may reduce inpatient LOS (Fernandez et al., 2018; Fernandez et al., 2013; Forder, 2009; Forder et al., 2018; Gaughan et al., 2017a; Gaughan et al., 2015; 2017b), home care explains little of the observed finding. Further analyses from the NHS, using similar data to those used in this chapter, find only a weak, non-statistically significant relationship between home care supply and inpatient LOS for those aged 65 years and over (Fernandez and Forder, 2008). Evidence from Switzerland by Gonçalves and Weaver (2017) shows that among those aged 65 years and over, no statistically significant reduction in LOS was observed in areas with more home care hours. However, the number of hospitalisations was shown to increase significantly as home care supply increased. Contrastingly, Costa-Font et al. (2018) show a policy to increase allowances for formal and informal home care resulted in a reduction in inpatient LOS by up to 30 per cent, reduced hospitalisation rates, and reduced hospital costs by 11 per cent (Costa-Font et al., 2018).

Building on the literature, we examine differences in home care supply across areas over time. Our analysis makes several contributions that extend previous research in this area. First, we use a large administrative database on the full population of public hospital inpatient discharges. In this context, we are not reliant on surveybased data using 12-month recall of hospital use. We more accurately measure hospital LOS and control for important patient-level characteristics, such as admission and discharge destination and diagnosis-related information. Second, we examine supply rather than allowances and examine public home care in a system where individuals do not require a co-payment in order to acquire public home care. Finally, in addition to examining average LOS, we estimate UQRs to examine whether home care supply has a bigger impact on reducing LOS for those with the longest LOS, many of whom would be classified as delayed discharges.

\subsubsection{Causal inference}

In this chapter, we try to estimate the causal effect of home care supply on inpatient LOS in Ireland. This is often difficult to do in the absence of a clear, natural experiment. We use differences in home care supply across areas and changes in supply over time within areas, controlling for a range of hospital-level and patientlevel characteristics. We use this modelling strategy to isolate the causal impact that supply of care for older people has on LOS. Using area-level long-term care supply is a common technique used in the literature to examine substitution effects (Costa-Font et al., 2018; Fernandez and Forder, 2015; Forder, 2009; Forder et al., 2019; Gaughan et al., 2017a; Gonçalves and Weaver, 2017).

We take advantage of the fact that supply of available home care differs significantly across areas and that potential home care supply available to 
individuals depends upon their residence in that area rather than the hospital in which they receive their inpatient treatment. These factors mean that there is no scope for patients to gain access to additional home care by choosing to attend a particular hospital. As noted earlier, Ireland has a large number of hospitals relative to the size of its population. Crucially (for our empirical strategy), the catchment areas of hospitals overlap in a number of areas (see Chapter 3 ). Therefore, for many hospitals, inpatients from a number of LHOs with different levels of home care supply will be included in the analyses at the hospital level. This means that our statistical analyses can compare LOS for patients within the same hospital but with differing levels of supply of home care in their LHOs, controlling for a range of other pertinent patient-level information. Our analyses can thereby more appropriately control for unobserved hospital-level effects, which may have an impact on treatment decisions, quality of care and, importantly, inpatient LOS.

One possible cause for concern is that the home care variable may be acting as a proxy for better non-acute service provision in an area more generally. For example, regions that saw increases in home care supply may have also seen increases in primary and community care supply, which may separately reduce inpatient LOS for both the older and younger populations. In order to test whether generalised improvements in non-acute care supply might explain our results, we undertake placebo tests to test the robustness of the link between home care supply and inpatient LOS in a younger population - aged 18-44 and 18-64. These younger populations are not targeted by the public home care schemes in Ireland; therefore, local supply of home care should have no bearing on their inpatient LOS. A lack of an effect on home care reducing LOS for a younger population will suggest that it is home care, rather than general improvements in non-acute care, that underpin our substitution results.

\subsection{RESULTS}

\subsubsection{Descriptive statistics}

Table 6.1 presents descriptive statistics. Overall mean LOS was 10.16 days, and the average age of discharges was 77.31 years. The majority (79 per cent) had a medical card and therefore free care during their inpatient stay. While all of the sample were admitted from home, not all were discharged home, with 12 per cent discharged to a long-stay facility. In the sample, stroke and hip fracture patients accounted for 2.85 per cent and 2 per cent of discharges respectively. 
TABLE 6.1 DESCRIPTIVE STATISTICS OF HIPE DATA FOR EMERGENCY INPATIENT DISCHARGES AGED $65+, 2012-2015$

\begin{tabular}{|c|c|c|}
\hline & Number of discharges & \\
\hline $\begin{array}{l}\text { Number of emergency inpatient } \\
\text { discharges }\end{array}$ & 304,005 & \\
\hline \multicolumn{3}{|l|}{ Year of discharge } \\
\hline 2012 & 77,452 & \\
\hline 2013 & 76,616 & \\
\hline 2014 & 73,504 & \\
\hline 2015 & 76,433 & \\
\hline \multirow[t]{2}{*}{ Dublin North } & 34,776 & \\
\hline & Mean & SD \\
\hline Length of stay & 10.16 days & 18.10 \\
\hline Age & 77.31 years & 7.69 \\
\hline Medical card & 0.79 & 0.41 \\
\hline Number of diagnoses & 5.14 & 3.46 \\
\hline Mean weighted Charlson Score & 1.25 & 1.83 \\
\hline \multicolumn{3}{|l|}{ Discharge destination } \\
\hline Home & 0.88 & - \\
\hline Long stay & 0.12 & - \\
\hline \multicolumn{3}{|l|}{ Marital status } \\
\hline Married & 0.49 & 0.50 \\
\hline Stroke & 0.0285 & - \\
\hline Hip fracture & 0.0202 & - \\
\hline
\end{tabular}

\subsubsection{Length of stay and inpatient bed days}

Figure 6.1 presents the cumulative inpatient bed days by LOS of the patient sample. Patients in the 90th LOS quantile, who make up 10 per cent of the sample, use 47.5 per cent of all inpatient bed days in a given year and have an average LOS of 46.6 days. This figure highlights that examining the longest stays using UQR may be most appropriate to understand how home care supply impacts LOS for the small proportion of inpatients who use a disproportionate amount of inpatient care. 
FIGURE 6.1 CUMULATIVE INPATIENT BED DAYS BY LENGTH OF STAY, EMERGENCY INPATIENT DISCHARGES AGED 65+, 2012-2015

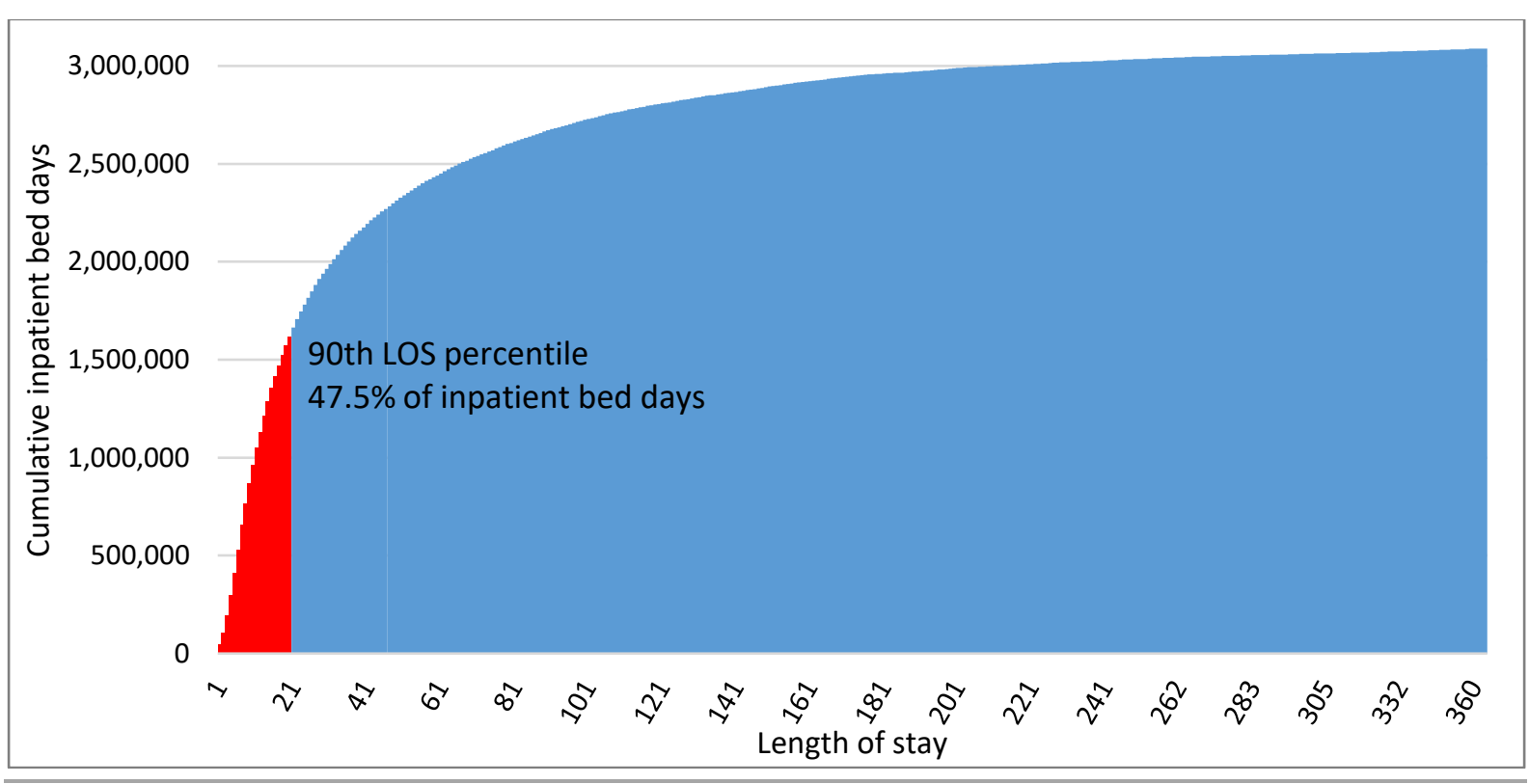

\subsubsection{Regression analyses}

Table 6.2 presents results from the linear and negative binomial regressions. Results show the impact of home care supply on average LOS. Coefficients on home care can be interpreted as elasticities. Across each model, a negative and statistically significant relationship between home care supply and LOS is observed. For the $65+$ sample, elasticities of 0.1 to 0.175 are observed; a 10 per cent increase in home care supply is associated with a 1 per cent ( 0.1 days) and 1.7 per cent $(0.2$ days) reduction in LOS. When home care hours are expressed in quintiles rather than as a continuous measure, patients in the top home care quintile have a 9.5 per cent (0.9 days) lower LOS than patients in the bottom home care quintile. Home care elasticities are similar across the $75+$ and $85+$ age groups; however, the result is not statistically significant for the $85+$ age group. 


\begin{tabular}{|c|c|c|c|c|c|}
\hline & \multicolumn{3}{|c|}{$65+$} & $75+$ & \multirow{2}{*}{ ares } \\
\hline & \multicolumn{2}{|c|}{ Ordinary least squares } & Negative binomial & Ordinary least squares & \\
\hline & (Ln LOS) & (Ln LOS) & (LOS) & (Ln LOS) & (Ln LOS) \\
\hline & (I) & (II) & (III) & (IV) & (V) \\
\hline Year trend & $0.011 * *$ & $0.013 * * *$ & $0.011^{* *}$ & $0.013^{* *}$ & 0.010 \\
\hline Married & $-0.116 * * *$ & $-0.116^{* * *}$ & $-0.153 * * *$ & $-0.108 * * *$ & $-0.058 * * *$ \\
\hline Medical card & $0.068 * * *$ & $0.068 * * *$ & $0.068^{* * *}$ & $0.051 * *$ & 0.038 \\
\hline Weighted Charlson score & $0.096 * * *$ & $0.097 * * *$ & $0.107 * * *$ & $0.108^{* * *}$ & $0.121 * * *$ \\
\hline Female & $0.020 * * *$ & $0.020 * * *$ & 0.010 & $0.022 * * *$ & $0.039 * * *$ \\
\hline \multicolumn{6}{|l|}{ Home care } \\
\hline Ln home care hours & $-0.102 * *$ & & $-0.175 * * *$ & $-0.129 * *$ & -0.097 \\
\hline \multicolumn{6}{|l|}{ Home care hour quintiles } \\
\hline Quintile 1 (Lowest) & & Base & & & \\
\hline Quintile 2 & & -0.032 & & & \\
\hline Quintile 3 & & -0.015 & & & \\
\hline Quintile 4 & & $-0.068 * * *$ & & & \\
\hline Quintile 5 (Highest) & & $-0.095^{* * *}$ & & & \\
\hline Clusters (hospitals) & 26 & 26 & 26 & 26 & 26 \\
\hline Observations & 304,005 & 304,005 & 304,005 & 183,586 & 60,463 \\
\hline Adjusted R squared & 0.276 & 0.276 & - & 0.243 & 0.211 \\
\hline Average length of stay & 10.16 days & 10.16 days & 10.16 days & 11.50 days & 13.29 days \\
\hline
\end{tabular}

Notes: $\quad$ Other covariates in models: DRG, hospital-level standardised bed capacity, age and age squared, season.

Sample includes patients admitted from home through an ED, not transferred to another hospital at discharge, alive at discharge. Clustered standard errors at hospital level. 
Home care supply may have differing effects on LOS across patients with different characteristics. Therefore, in Figure 6.2, we interact home care with sex, marital status, private discharge status and weighted Charlson comorbidity score to test whether increased home care supply may reduce LOS at a greater rate for females (versus males), married patients, private patients or sicker patients with more comorbidities. ${ }^{42}$ Results are based upon the linear regression model from Column I in Table 6.2.

While LOS is longer for females, home care supply appears to have a stronger impact on reducing LOS for females than males. Home care appears to have a similar effect for those who are married and not married. Interestingly, no difference is observed across public and private patients. Finally, it appears that home care supply has a slightly larger impact in reducing LOS for patients with a higher weighted Charlson comorbidity score; in other words, sicker patients.

42 Regarding private discharge, public/private discharge status is included in the regression instead of medical card status to estimate findings for this figure. 


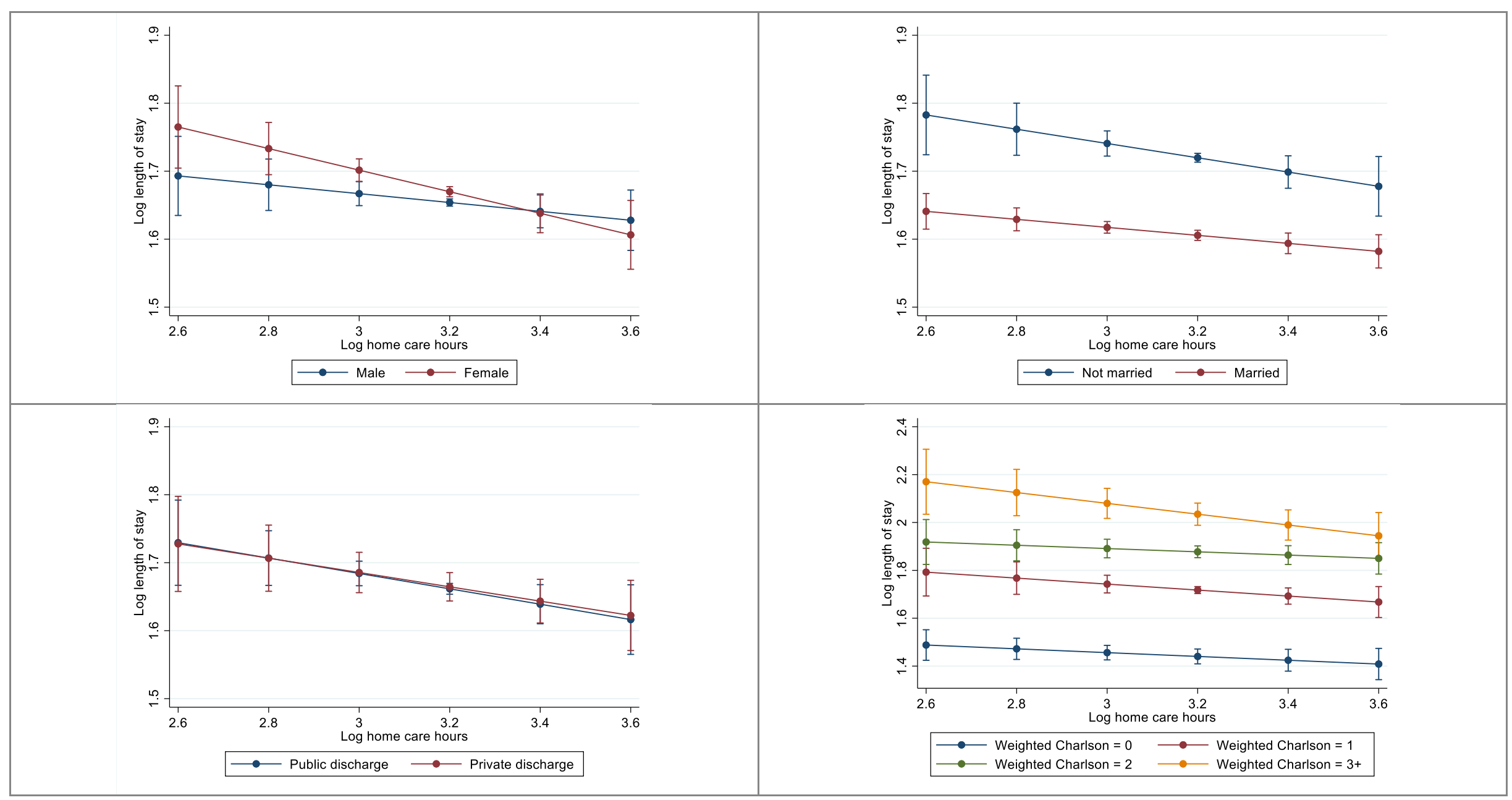

Notes: $\quad$ Ordinary least squares regressions based on Table 6.2, Column I. Covariates in models: year, married, medical card, weighted Charlson score, hospital FES, DRG, hospital-level standardised bed

capacity, age and age squared, season.
Sample: patients admitted from home through an ED, not transferred to another hospital at discharge, alive at discharge. Clustered standard errors at hospital level. 
The results presented in Table 6.2 show that home care is associated with lower LOS on average. However, examining effects on average LOS may mask some differences in the impact that home care has across patients with differing LOS, especially those with long LOS who use a disproportionate amount of inpatient bed days. Those with longer LOS may be more amenable to home care as their major surgical/medical treatment has already been provided, and many bed days subsequent to this are being used for rehabilitative or post-treatment care, much of which could be provided at home subject to the availability of non-acute services and a package of home care.

In Figure 6.3, we present results from the UQR where the impact of home care supply is examined across LOS quantiles. Here, home care has a stronger negative association with reducing LOS for patients with longer LOS. Large negative coefficients are seen for those with an LOS greater than 21 days. For patients with longer stays, a 10 per cent increase in home care supply is associated with a 0.3 days reduction in LOS for those in the 90th percentile (equating to a 1.3 per cent reduction in LOS) and a 0.6 days reduction for those in the 95th percentile (equating to a 1.7 per cent reduction in LOS). In this regard, home care supply is associated with a greater reduction in LOS for patients who are more likely to be characterised as delayed discharges.

FIGURE 6.3 UNCONDITIONAL QUANTILE REGRESSION FOR EMERGENCY INPATIENT DISCHARGES AGED 65+, 2012-2015

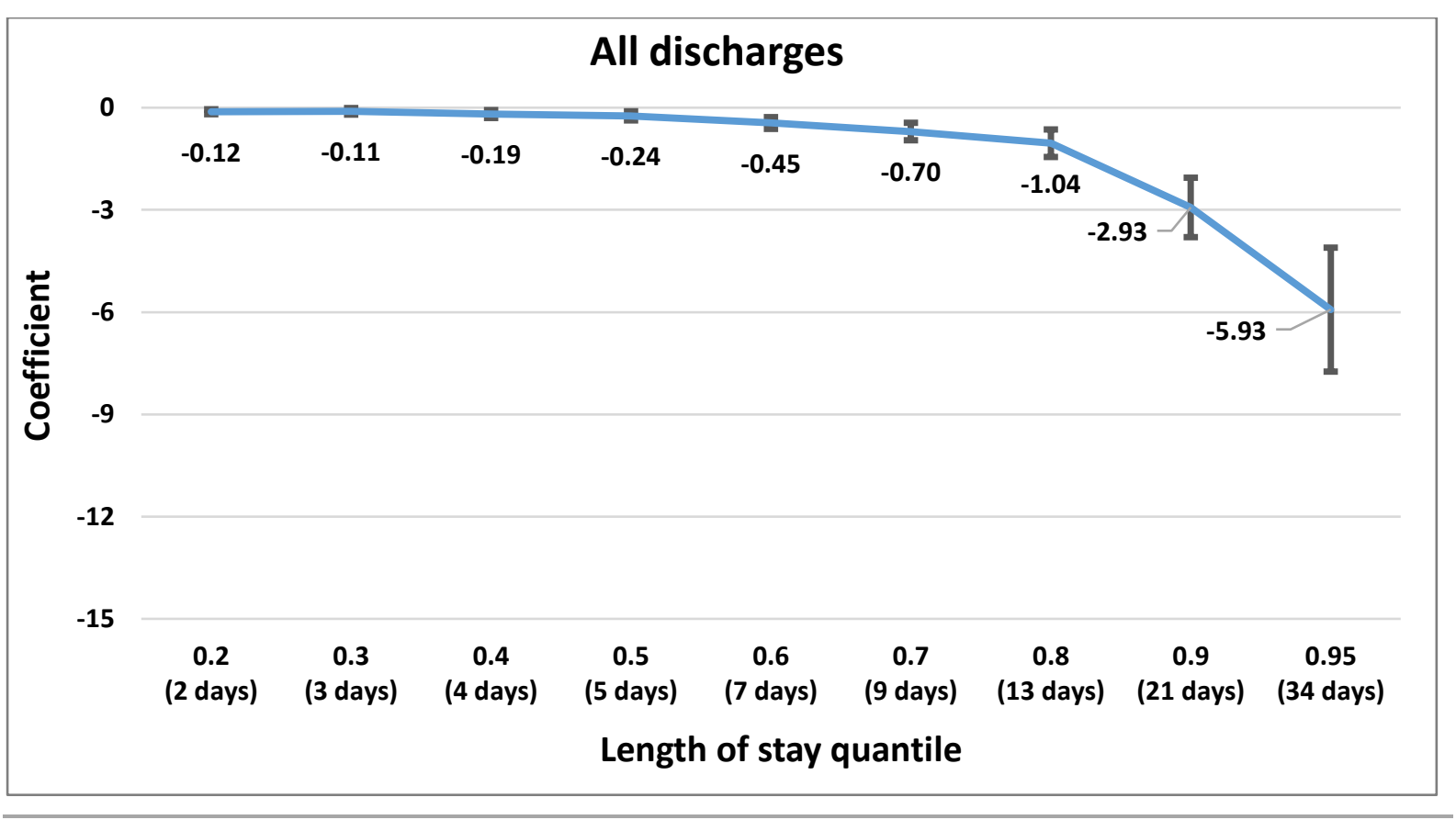


Home care per capita in Dublin North increased by 50 per cent between 2012 and 2015 , a much greater rate than the increase observed in Ireland on average. This increase was likely a policy response to the low relative supply of home care in the area and the long LOS in public hospitals in Dublin North (see Table 6.3). Examining Dublin North will also allow us to test the theory that the relationship between home care and LOS might be non-linear, whereby home care supply in an area above some threshold level leads to significantly greater response in reducing LOS. We have used a categorical representation of home care hours to allow for possible non-linearities at individual discharge level, but there might be threshold effects that operate at area level as well; for example, to do with policies affecting discharge timing in general.

Table 6.3 presents results from the linear and negative binomial regressions for Dublin North discharges. Results follow a similar pattern as seen in the countrywide analyses, with a negative and statistically significant relationship between home care supply and LOS observed, though elasticities are larger than seen for the countrywide analyses. For the 65 years and over sample, elasticities of approximately 0.27 are observed; a 10 per cent increase in home care supply is associated with a 2.7 per cent ( 0.4 days) reduction in LOS. The coefficient is larger for those aged $75+$, where a 10 per cent increase in public home care provision is associated with a 4.2 per cent ( 0.7 days) reduction in LOS. Home care elasticities are large in the 85+ models; however they are not statistically significant. Results on other variables including sex, marital status and weighted Charlson morbidity scores follow a similar pattern to the Ireland analyses. 
TABLE 6.3 DETERMINANTS OF LENGTH OF STAY FOR EMERGENCY INPATIENT DISCHARGES AGED 65+, DUBLIN NORTH, 2012-2015

\begin{tabular}{|c|c|c|c|c|}
\hline & \multicolumn{2}{|c|}{$65+$} & \multirow{2}{*}{ Ordinary least squares } & \multirow{2}{*}{$\frac{85+}{\text { Ordinary least squares }}$} \\
\hline & Ordinary least squares & Negative binomial & & \\
\hline & (Ln LOS) & (LOS) & (Ln LOS) & (Ln LOS) \\
\hline & (I) & (II) & (III) & (IV) \\
\hline Year trend & 0.010 & -0.003 & 0.024 & 0.012 \\
\hline Married & $-0.109 * * *$ & $-0.147 * * *$ & $-0.084 * * *$ & -0.006 \\
\hline Medical card & $0.135^{* * *}$ & $0.141^{* * *}$ & $0.136^{* * *}$ & $0.109 * * *$ \\
\hline Weighted Charlson score & $0.136 * * *$ & $0.156 * * *$ & $0.157 * * *$ & $0.188 * * *$ \\
\hline Female & 0.016 & -0.006 & $0.029 *$ & $0.084 * * *$ \\
\hline \multicolumn{5}{|l|}{ Home care } \\
\hline Ln home care hours & $-0.271 * * *$ & $-0.279 *$ & $-0.420 * * *$ & -0.338 \\
\hline Clusters (hospitals) & - & - & - & - \\
\hline Observations & 34,776 & 34,776 & 21,195 & 6,833 \\
\hline Adjusted R Squared & 0.303 & - & 0.266 & 0.211 \\
\hline Average length of stay & 13.92 days & 13.92 days & 16.21 days & 19.28 days \\
\hline
\end{tabular}

Notes: Other covariates in models: DRG, hospital-level standardised bed capacity, age and age squared, season.

Sample: Patients admitted from home through an ED in Dublin North and with an address in Dublin North, not transferred to another hospital at discharge, alive at discharge. Clustered standard errors at hospital level.

Home care is not included in quintiles as only one area is examined. 
Figure 6.4 presents results from an unconditional quantile regression on the relationship between home care and LOS across the LOS distribution for Dublin North. A pattern similar to the Ireland analyses is observed, but the size of the coefficients once more are larger for Dublin North. No statistically significant relationship between LOS and home care supply is observed prior to the 80th percentile. However, at the top of the distribution, a 10 per cent increase in home care provision is associated with a 2-day reduction in LOS for those in the 90th percentile (equating to a 6.9 per cent reduction in LOS) and a 5.2 days reduction for those in the 95th percentile (equating to a 10.5 per cent reduction in LOS).

FIGURE 6.4 UNCONDITIONAL QUANTILE REGRESSION ON EMERGENCY INPATIENT DISCHARGES AGED 65+ DUBLIN NORTH, 2012-2015

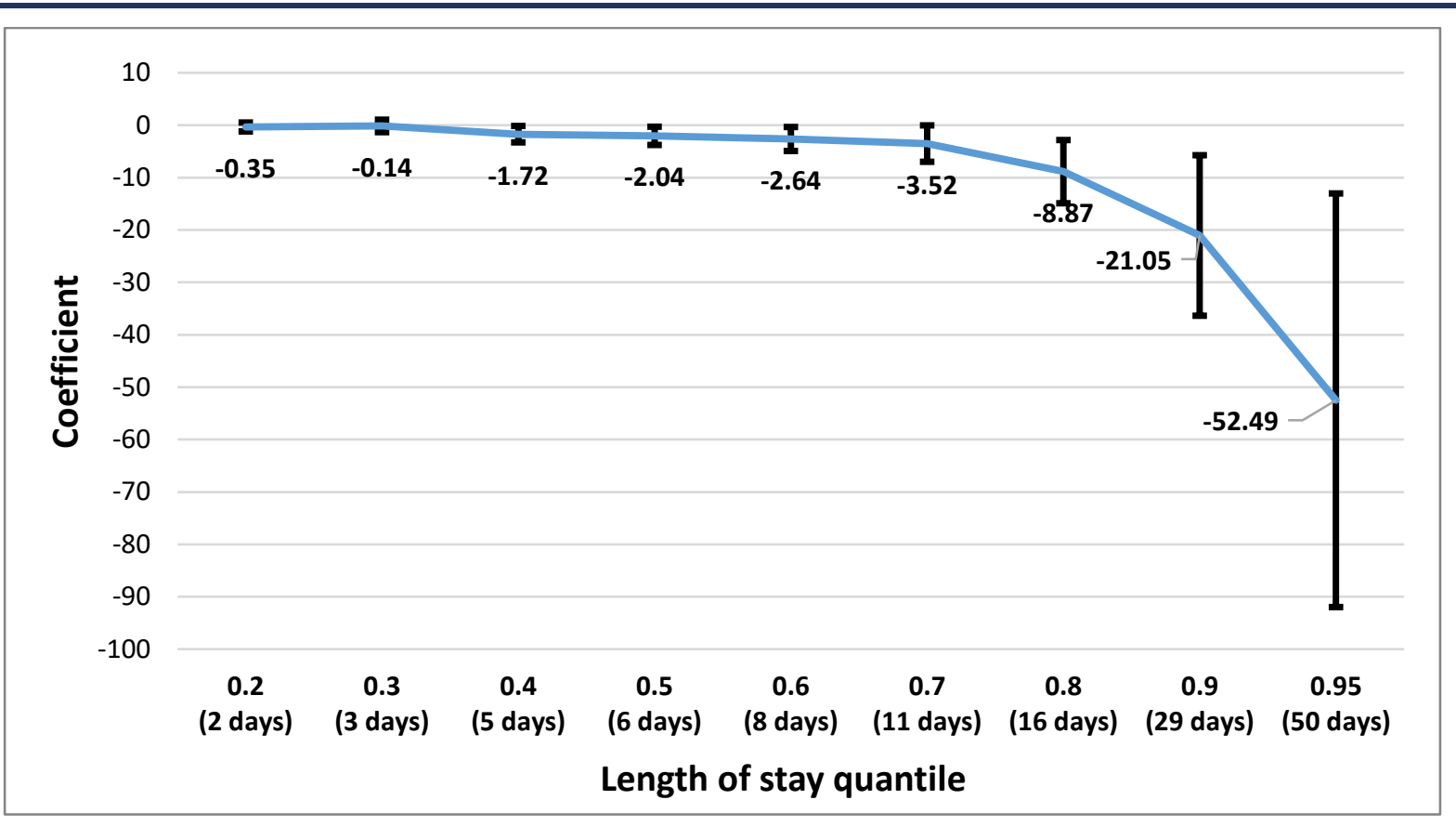

Differences between Dublin North and Ireland more generally, regarding the impact of home care, are quite large. In Table 6.4, we try to scrutinise whether these results are borne out by changes in LOS over time. This table shows that while LOS in the top quantiles in Ireland increased slightly between 2012 and 2015, large reductions were seen in Dublin North in line with what is predicted in the models. LOS in the 90th and 95th quantile in Dublin North reduced by 3 days (31 days to 28 days) and 11 days (56 days to 45 days) respectively. This suggests that the larger coefficients observed in the Dublin North analyses in this chapter are qualitatively accurate. 
TABLE 6.4 EMERGENCY INPATIENT LENGTH OF STAY QUANTILES, IRELAND AND DUBLIN NORTH, 2012 AND 2015

\begin{tabular}{|l|c|c|c|c|}
\hline & \multicolumn{2}{|c|}{ Ireland } & \multicolumn{2}{c|}{ Dublin North } \\
\hline Quantile & $\mathbf{2 0 1 2}$ & $\mathbf{2 0 1 5}$ & $\mathbf{2 0 1 2}$ & $\mathbf{2 0 1 5}$ \\
$(\mathrm{n}=77,452)$ & $(\mathrm{n}=76,433)$ & $\mathrm{n}=9,117)$ & $\mathrm{n} / \mathrm{a}$ \\
\hline <20th & $\mathrm{n} / \mathrm{a}$ & $\mathrm{n} / \mathrm{a}$ & $\mathrm{n} / \mathrm{a}$ & $\mathbf{2}$ \\
\hline 20th & 2 days & 2 days & 3 & 3 \\
\hline 30th & 3 & 3 & 5 & 5 \\
\hline 40th & 4 & 4 & 6 & 6 \\
\hline 50th & 5 & 6 & 8 & 8 \\
\hline 60th & 7 & 7 & 11 & 11 \\
\hline 70th & 9 & 9 & 17 & 16 \\
\hline 80th & 12 & 13 & 31 & 28 \\
\hline 90th & 21 & 22 & 56 & 45 \\
\hline 95th & 33 & 35 & & 5 \\
\hline
\end{tabular}

\section{Stroke and hip fracture patients}

Stroke and hip fracture patients may be more amenable to home care reducing their LOS than many other types of patients. Table 6.5 and Table 6.6 present results from the linear and negative binomial regressions examining the impact of home care supply on LOS. Due to smaller numbers, it was not possible to undertake UQRs for these patient groups. Table 6.5 shows that a negative and statistically significant relationship between home care supply and LOS is observed for stroke patients. Within the $65+$ group, a 10 per cent increase in home care supply is associated with a 2.7 per cent ( 0.67 days) and 3.1 per cent ( 0.76 days) reduction in LOS. When public home care supply in patient area of residence is included as quintiles, stroke patients in the top home care quintile have LOS 19.6 per cent lower than patients in the bottom home care quintile.

The size of the relationship between home care and stroke LOS also increases across age, with the impact of public home care provision having a larger impact at older ages; a 10 per cent increase in public home care provision is related to a 3.3 per cent ( 1 day) and 3.8 per cent ( 1.4 days) reduction in LOS for those aged 75 years and over and 85 years and over, respectively. 
TABLE 6.5 DETERMINANTS OF LENGTH OF STAY FOR EMERGENCY INPATIENT STROKE DISCHARGES AGED 65+, 2012-2015

\begin{tabular}{|c|c|c|c|c|c|}
\hline & \multicolumn{3}{|c|}{$65+$} & $75+$ & $85+$ \\
\hline & \multicolumn{2}{|c|}{ Ordinary least squares } & \multirow{2}{*}{$\begin{array}{c}\text { Negative binomial } \\
\text { (LOS) }\end{array}$} & \multicolumn{2}{|c|}{ Ordinary least squares } \\
\hline & (Ln LOS) & (Ln LOS) & & (Ln LOS) & (Ln LOS) \\
\hline & (I) & (II) & (III) & (IV) & (V) \\
\hline Year trend & 0.000 & -0.001 & -0.009 & 0.012 & 0.009 \\
\hline Married & $-0.128 * * *$ & $-0.128 * * *$ & $-0.164 * * *$ & $-0.139 * * *$ & $-0.093^{*}$ \\
\hline Medical card & 0.028 & 0.027 & 0.041 & 0.009 & -0.044 \\
\hline Weighted Charlson score & $0.044 * * *$ & $0.044 * * *$ & $0.043^{* * *}$ & $0.041 * *$ & 0.009 \\
\hline Female & -0.004 & -0.004 & -0.027 & 0.018 & 0.060 \\
\hline \multicolumn{6}{|l|}{ Home care } \\
\hline Ln home care hours & $-0.270 * * *$ & & $-0.307 * * *$ & $-0.331 * * *$ & $-0.382 *$ \\
\hline \multicolumn{6}{|l|}{ Home care hour quintiles } \\
\hline Quintile 1 (Lowest) & & Base & & & \\
\hline Quintile 2 & & -0.079 & & & \\
\hline Quintile 3 & & -0.044 & & & \\
\hline Quintile 4 & & $-0.224 * * *$ & & & \\
\hline Quintile 5 (Highest) & & $-0.196 * *$ & & & \\
\hline Clusters (hospitals) & 26 & 26 & 26 & 26 & 26 \\
\hline Observations & 8,533 & 8,533 & 8,533 & 5,537 & 1,921 \\
\hline Adjusted R Squared & 0.281 & 0.281 & & 0.264 & 0.232 \\
\hline Average length of stay & 24.91 days & 24.91 days & 24.91 days & 27.92 days & 31.05 days \\
\hline
\end{tabular}

Notes: $\quad$ Other covariates in models: hospital-level standardised bed capacity, age and age squared, season, stroke severity.

Sample: Patients with primary or secondary stroke diagnosis, admitted from home through an ED, not transferred to another hospital at discharge, alive at discharge. Clustered standard errors at hospital level. 
Table 6.6 presents results from linear and negative binomial regressions for hip fracture patients. A negative and statistically significant relationship between home care supply and LOS is observed. Within the 65+ group, a 10 per cent increase in home care supply is associated with a 1.6 per cent ( 0.37 days) reduction in LOS for hip fracture patients. When public home care supply in patient area of residence is included as quintiles, hip fracture patients in the top home care quintile have a 13 per cent lower LOS than patients in the bottom home care quintile. The size of the relationship between home care and hip fracture LOS is highest in those aged 85 years and over, where a 10 per cent increase in public home care supply is related to a 2.8 per cent ( 0.7 days) reduction in LOS. 


\begin{tabular}{|c|c|c|c|c|c|}
\hline & \multicolumn{3}{|c|}{$65+$} & $75+$ & \multirow{2}{*}{ ares $85+$} \\
\hline & \multicolumn{2}{|c|}{ Ordinary least squares } & Negative binomial & Ordinary least squares & \\
\hline & (Ln LOS) & (Ln LOS) & (LOS) & (Ln LOS) & (Ln LOS) \\
\hline & (I) & (II) & (III) & (IV) & (V) \\
\hline Year trend & -0.003 & 0.000 & 0.000 & -0.005 & -0.003 \\
\hline Married & 0.007 & 0.007 & $-0.130 * * *$ & 0.033 & $0.069 *$ \\
\hline Medical card & $0.104 * * *$ & $0.106^{* * *}$ & 0.059 & $0.122 * * *$ & $0.113 * *$ \\
\hline Weighted Charlson score & $0.168 * * *$ & $0.168^{* * *}$ & $0.201^{* * *}$ & $0.165^{* * *}$ & $0.150 * * *$ \\
\hline Female & $-0.079 * * *$ & $-0.079 * * *$ & $-0.146 * * *$ & $-0.038^{*}$ & 0.014 \\
\hline \multicolumn{6}{|l|}{ Home care } \\
\hline Ln home care hours & $-0.158 * *$ & & $-0.154 *$ & $-0.165^{*}$ & $-0.283^{*}$ \\
\hline Home care hour quintiles & & Base & & & \\
\hline Quintile 1 (Lowest) & & 0.014 & & & \\
\hline Quintile 2 & & 0.041 & & & \\
\hline Quintile 3 & & $-0.102 * * *$ & & & \\
\hline Quintile 4 & & $-0.130 * * *$ & & & \\
\hline Quintile 5 (Highest) & & 0.014 & & & \\
\hline Clusters (hospitals) & 26 & 26 & 26 & 26 & 26 \\
\hline Observations & 6,136 & 6,136 & 6,136 & 4,794 & 2,327 \\
\hline Adjusted R Squared & 0.146 & 0.147 & & 0.125 & 0.107 \\
\hline Average length of stay & 23.26 days & 23.26 days & 23.26 days & 24.74 days & 26.82 days \\
\hline
\end{tabular}

Notes: Other covariates in models: Hospital-level standardised bed capacity, age and age squared, season.

Sample: Patients with primary or secondary hip fracture diagnosis, admitted from home through an ED, not transferred to another hospital at discharge, alive at discharge.

Clustered standard errors at hospital level. 


\subsubsection{Placebo tests}

In this chapter, we use differences in supply of home care across areas and over time, controlling for a range of patient-level characteristics, to estimate the relationship between home care and earlier discharge for older inpatients from public hospitals. However, regions with better home care supply may also have better non-acute and social care facilities more generally, and changes in the provision of home care may move in line with other service improvements. Therefore, the home care variable may be acting as a proxy for better non-acute service provision in a region. In order to test whether broader changes in non-acute care supply might explain our results, we test the robustness of the link between home care supply and inpatient LOS in a younger population, a population much less likely to avail of public home care services such as home care packages (HCPs), but likely to benefit from improvements to non-acute care more generally.

Table 6.7 presents results from the linear regression analysis of the determinants of LOS for those aged 18-44 years and 18-64 years, using the same sets of confounding variables as in the models reported above. Coefficients on marital status, weighted Charlson score medical card status and sex all show similar results to those reported in Table 6.2 for those aged 65 years and over. In the 0-44 and 18-44 age groups, home care does not have a statistically significant association with LOS. Home care shows a small negative and statistically significant (at the 90 per cent level) association for the 18-64 age group. Further analysis shows that this result is driven by those aged 45 years and older. As approximately 10 per cent of all home care provision is provided to those aged 45-64, this small and marginally significant association could be due to this group's limited use of home care services. In Dublin North, where home care supply saw the largest increase, no statistically significant coefficient on the home care variable is observed for the 18-44 and 18-64 age groups. Similarly, Figure A.6 in the appendix provides results from unconditional quantile regressions showing no relationship between home care and LOS across the LOS distribution for in those aged 18-44 years.

Results from these placebo analyses show that the findings of a negative relationship between home care supply and inpatient LOS in inpatients aged 65 years and over are unlikely to be driven by an underlying, omitted, non-acute care variable. Rather, the coefficients on home care are likely to relate to effects of home care supply. 
TABLE 6.7 DETERMINANTS OF LENGTH OF STAY FOR EMERGENCY INPATIENT DISCHARGES AGED UNDER 65 YEARS, IRELAND AND DUBLIN NORTH, 2012-2015

\begin{tabular}{|l|c|c|c|c|}
\hline & \multicolumn{2}{|c|}{ Ireland } & \multicolumn{2}{c|}{ Dublin North } \\
\hline & $\mathbf{1 8 - 4 4}$ years & $\mathbf{1 8 - 6 4}$ years & $\mathbf{1 8 - 4 4}$ years & $\mathbf{1 8 - 6 4}$ years \\
\hline & (Ln LOS) & (Ln LOS) & (Ln LOS) & (Ln LOS) \\
\hline Year trend & (I) & (II) & (III) & (IV) \\
\hline Married & 0.002 & 0.004 & $-0.023^{* * *}$ & $-0.020^{* * *}$ \\
\hline Medical card & $-0.056^{* * *}$ & $-0.076^{* * *}$ & $-0.041^{* * *}$ & $-0.057^{* * *}$ \\
\hline $\begin{array}{l}\text { Weighted Charlson } \\
\text { score }\end{array}$ & $0.058^{* * *}$ & $0.074^{* * *}$ & $0.101^{* * *}$ & $0.117^{* * *}$ \\
\hline Female & $0.098^{* * *}$ & $0.083^{* * *}$ & $0.082^{* * *}$ & $0.085^{* * *}$ \\
\hline Home care & $0.020^{* * *}$ & $0.016^{* * *}$ & 0.010 & 0.012 \\
\hline $\begin{array}{l}\text { Ln home care hours } \\
\text { Clusters (hospitals) }\end{array}$ & $-\mathbf{0 . 0 2 2}$ & $-0.034 *$ & & $-\mathbf{0 . 0 0 9}$ \\
\hline Observations & 26 & 26 & $-\mathbf{0 . 0 0 2}$ & - \\
\hline Adjusted R squared & 204,755 & 399,329 & - & 44,202 \\
\hline Average LOS & 0.319 & 0.355 & 23,355 & 0.412 \\
\hline
\end{tabular}

Notes: Linear regression analyses. Other covariates in models: DRG, hospital-level standardised bed capacity, age and age squared, season.

Sample: Patients admitted from home through an ED, not transferred to another hospital at discharge, alive at discharge.

Clustered standard errors at hospital level for Ireland sample.

\subsection{CONCLUSIONS}

Findings from this chapter show that home care can reduce inpatient LOS for older patients. When average LOS across all emergency inpatients admitted from home aged 65 years and over, both linear and negative binomial regressions show a small substitution effect. These results find that, ceteris paribus, a 10 per cent increase in home care supply per capita (similar to the increase observed in Ireland between 2012 and 2015) is associated with an approximate 1-1.7 per cent reduction in average inpatient LOS. The findings of a small negative relationship between home care supply and average inpatient LOS is consistent with previous evidence from the NHS (Fernandez and Forder, 2008) and Switzerland (Gonçalves and Weaver, 2017). Another way of illustrating the scale of effects shown in this chapter is to say that a 10 per cent increase in home care per capita (1.5 million hours per annum) is associated with a reduction of up to 14,700 inpatient bed days, which equates with 40 more inpatient beds available to the system daily.

Examining those with long LOS (delayed discharges), a larger substitutive effect of home care supply is found. Results from UQR analyses show that for those in the 90th percentile of LOS, a 10 per cent increase in per capita home care supply is associated with 0.3 fewer hospital days. The relationship is stronger in the 95th percentile where a 10 per cent increase in per capita home care supply is associated with a 1.2 days reduction in LOS. 
The results also differ when we examine an area that received a very large increase in home care supply between 2012 and 2015. In Dublin North, an even larger effect of home care on reducing LOS is observed, suggesting that assuming linearity in substitution effects could be misleading. When average LOS is examined, ceteris paribus, a 10 per cent increase in home care supply per capita is associated with an approximate 2.7 per cent reduction in average inpatient LOS for inpatients aged 65 years and over. ${ }^{43}$ Imposing these results on the countrywide sample would result in 40,000 fewer inpatient bed days per annum, and 110 more inpatient beds available to the system daily. Results from UQR analyses in Dublin North show that for those in the 90th percentile of LOS, a 10 per cent increase in per capita home care supply is associated with 2 fewer days spent in hospital. Again, the relationship in Dublin North between LOS and home care is stronger in the 95th percentile, where a 10 per cent increase in per capita home care supply is associated with a 5.2 days reduction in LOS.

The large effects observed in Dublin North comply with the large actual reductions in LOS found in Dublin North hospitals in recent years. As we show, LOS in the 90th and 95th quantile in Dublin North reduced by 3 days ( 31 days to 28 days) and 11 days ( 56 days to 45 days) between 2012 and 2015. While these reductions may be a consequence of a number of factors, results of this chapter do suggest that the increases in home care supply contributed to much of these reductions.

Results for stroke and hip fracture patients, two groups of patients that are more likely to benefit from home care services, also show a much larger substitution effect than in the general patient sample. Linear and negative binomial regressions show that a 10 per cent increase in home care supply is associated with a 0.75 and 0.35 day reduction in average LOS respectively. Reducing inpatient care by this amount would save 3 per cent and 1.5 per cent of bed days for those aged 65 years and over with stroke and hip fracture respectively in 2015. Due to the low number of patients, it was not possible to examine whether home care supply had a stronger relationship with reduced LOS for stroke and hip fracture patients with longer LOS; it is expected the relationship would follow that observed for all patients, whereby the largest substitution effects are seen for delayed discharges.

Examining the homogeneity of the substitution effects on average LOS across demographic groups (gender, marital status and Charlson comorbidity illness score), we see some small differences. Female patients aged 65 years and over have longer LOS than male patients in general. Results show a sharper reduction in LOS for females than males as LOS increases. No differences were observed across married and not-married patients, or between public and private patients. Previous evidence shows that widowed or unmarried adults have the highest risks of long-term care admission (Thomeer et al., 2016), likely a proxy of low informal

43 A larger elasticity of 0.42 is estimated for those aged 75 years and over. 
care supply. Additionally, those living alone in Ireland were found to be 2.6 times more likely to receive formal home care than those who lived with others (Murphy et al., 2015).

These results should be interpreted in the context that some of those aged 65 years and over in this study may not be amenable to, or require, home care in order to be discharged earlier. Furthermore, at any moment in time, the amount of home care supply available for use may be small (as compared to overall supply in an area). Therefore, these factors may mean that we are underestimating the true impact of home care provision. This may also explain why the coefficients in Dublin North are much stronger where a substantial increase in home care supply occurred over a short period of time, and therefore at any given moment, a larger proportion of home care may be available as compared to the rest of Ireland. 



\section{CHAPTER 7}

\section{Does long-term residential care reduce public hospital inpatient length of stay and delayed discharges?}

\subsection{SCOPE OF THE CHAPTER}

In this chapter, we examine the relationship between inpatient length of stay (LOS) and long-term residential care (LTRC) bed supply in Ireland for the older population between 2012 and 2015. Section 7.2 details the question examined and the patients included in the study. Section 7.3 summarises the background to the analyses. Section 7.4 presents the findings and Section 7.5 concludes.

\subsection{QUESTION}

In the analysis, we estimate the impact of supply of LTRC beds on emergency inpatient LOS in public hospitals in Ireland amongst patients aged 65 years and over. We also examine the impact of LTRC supply on those with long LOS; a group potentially disproportionately made up of those with delayed discharges. We use variations in LTRC bed supply across areas and over time as a means to estimate associations between LTRC supply and inpatient LOS. We assume that LTRC supply for a patient is based predominantly upon the supply within their Local Health Office (LHO). However, in contrast to home care, patients can choose to use LTRC outside of their immediate LHO.

In this analysis, as we seek to examine the impact LTRC bed supply has on LOS for the older inpatient population, we do not include patients in our sample who were already in an LTRC centre prior to their admission to hospital. (It is assumed that these patients, when fit for discharge, will be discharged back to their centre, and the supply of LTRC beds will have little impact on their LOS. ${ }^{44}$ We examine the impact LTRC bed supply has on patients who are admitted from home. The sample included is similar to that examined in Chapter 6; however, we also include patients discharged to other facilities.

We undertake a series of sub-analyses in this chapter to examine the robustness of results across different patient populations. First, we examine those patients who are discharged to an LTRC centre separately, to test the hypothesis that the impact of LTRC bed supply might have a greater impact on reducing LOS for this group of patients. (Evidence in Chapter 5 also shows that these patients have a very long LOS, on average.) Second, we examine three specific groups of patients seen in the literature as most amenable to LTRC supply: stroke; hip fracture; and 
patients with Alzheimer's/dementia (Connolly and O'Shea, 2015; Gaughan et al., 2015; Huckfeldt et al., 2014). Patients with Alzheimer's disease/dementia account for a large proportion of LTRC residents (Cahill et al., 2015). Patients with a primary or secondary diagnosis of stroke are identified using ICD-10 classification codes 160 , 161, 163 and 164, resulting in 8,671 stroke discharges. Patients with a primary diagnosis of hip fracture patients were identified using ICD-10 codes S7200, S7201 and 57220 , resulting in 6,136 hip fracture discharges. Patients with a primary or secondary diagnosis of Alzheimer's disease or dementia were identified using ICD10 codes G300, F01, F02 and F03, resulting in 6,136 Alzheimer's/dementia discharges ${ }^{45}$ We also examine those aged 75 years and over and 85 years and over separately, as LTRC utilisation rates are much higher in the over 75 and over 85 age groups (Wren et al., 2017). Heterogeneity in the effect of LTRC on LOS is examined across specific demographic groups including gender, marital status, public/private status and comorbidity.

We estimate linear regression and negative binomial models to examine the effect of LTRC supply on average LOS. As in Chapter 6 , in order to examine whether the association between LTRC supply and LOS varies across the distribution of LOS, we also estimate unconditional quantile regressions (UQRs) on LOS. We control for a range of other confounders, including: day of admission; year of admission; admission source; discharge destination; age and age squared; weighted Charlson comorbidity score (linear and squared); sex; marital status; specific diagnosisrelated group (DRG); and medical card status. Finally, to control for unobserved hospital-level factors that may affect results, we include hospital fixed effects. A standardised beds measure at the hospital-month level is also included to account for bed capacity changes in each hospital over the period (see Chapter 3). Finally, as we showed in Chapter 6 that an individual's LOS and discharge destination may be affected by home care supply, we control for home care hours per capita in each LHO for each year. This means we examine the impact of LTRC supply on inpatient LOS, controlling for both 'push' (inpatient bed supply) and alternative 'pull' (home care supply) factors.

\subsection{BACKGROUND}

As discussed in Chapter 3, there is relatively limited evidence internationally on substitution between acute care and long-term care (namely, social care or care for older people). However, where evidence has demonstrated such a link, the substitution effects observed for acute care are driven predominantly by LTRC supply or expenditure as opposed to home care supply or expenditure. Evidence shows a clear link between LTRC and reduced hospital use (Fernandez et al., 2018; 
Fernandez et al., 2013; Forder, 2009; Forder et al., 2018; Gaughan et al., 2017a; Gaughan et al., 2015; 2017b). Forder (2009) found that increasing long-term care home expenditure in England by $£ 1$ reduced acute hospital expenditure by $£ 0.35$. Using data from the NHS, Gaughan et al. (2015) found that inpatient LOS, and delayed discharged in particular, respond to LTRC bed supply. The authors found that a 10 per cent increase in LTRC beds reduces delayed discharges (where need for long-term care is given as the reason for the discharge delay) by between 6 per and 9 per cent.

In this chapter, we use similar data and methodologies to those used in the existing literature in order to examine LTRC supply at the regional level. We use a large administrative database on the full population of public hospital inpatient discharges. In this context, we are not reliant on survey-based data using 12 month-recall of hospital use. We may more accurately measure hospital LOS and control for important patient-level characteristics such as admission and discharge destination and diagnosis-related information. Second, we examine the impact of LTRC bed supply in a system where a statutory scheme (Fair Deal) finances 75 per cent of LTRC residents, subject to some co-payments, with others financed by legacy public schemes. In this context, the estimated substitution effects are closely identified with a specific state policy instrument. However, due to the lack of consistent data prior to the establishment of Fair Deal (pre-2009), we are unable to explicitly isolate the impact of the new policy on patient outcomes. Finally, in addition to examining average LOS, we estimate UQRs to examine whether LRTC supply has a bigger impact on reducing LOS for those with the longest LOS, many of whom are thought to be affected by delayed discharges.

Unlike the previous chapter, here, as a consequence of the (limited) data available and the manner in which individuals can choose their LTRC centre, it is much more difficult to examine causal effects. Nevertheless, the variation in supply across areas over time, controlling for a range of patient-level factors, will allow for a meaningful result to be estimated. Since areas with better LTRC supply may also have better non-acute and social care facilities more generally, and LTRC may be acting as a proxy for better non-acute service supply in an area, we also undertake a placebo test to examine whether LTRC bed supply impacts patients admitted from long-stay facilities. Discharge timing for such patients should not be directly affected by the supply of LTRC places, since they should already have a place at the time of admission. 


\subsection{RESULTS}

\subsubsection{Descriptive statistics}

Table 7.1 presents descriptive statistics for our sample of 333,928 discharges. Overall, mean LOS was 10.51 days, and the average age of discharges was 77.36 years. The majority, 78 percent, had a medical card and therefore received care for free during their inpatient stay. While all of the sample were admitted from home, 20 per cent were discharged to a long-stay facility, transferred to another hospital, or otherwise discharged. Stroke, hip fracture and Alzheimer's/dementia patients accounted for 3.3 percent, 2.8 per cent, and 5.1 per cent of discharges respectively. Average LOS for those ultimately discharged to a long-stay facility (LTRC centre) over three times longer than for those discharged home at 26.3 days.

TABLE 7.1 DESCRIPTIVE STATISTICS OF HIPE DATA FOR EMERGENCY INPATIENT DISCHARGES AGED $65+, 2012-2015$

\begin{tabular}{|c|c|c|}
\hline & Number of discharges & \\
\hline N. emergency inpatient discharges & 333,928 & \\
\hline \multicolumn{3}{|l|}{ Year of discharge } \\
\hline 2012 & 84,265 & \\
\hline 2013 & 83,863 & \\
\hline 2014 & 81,621 & \\
\hline \multirow[t]{2}{*}{2015} & 84,179 & \\
\hline & Mean & SD \\
\hline Length of stay & 10.51 days & 18.47 \\
\hline Age & 77.36 years & 7.70 \\
\hline Medical card & 0.78 & 0.41 \\
\hline Number of diagnoses & 5.27 & 3.55 \\
\hline Mean weighted Charlson score & 1.25 & 1.83 \\
\hline \multicolumn{3}{|l|}{ Discharge destination } \\
\hline Home & 0.80 & \\
\hline Long stay & 0.11 & \\
\hline Transfer & 0.08 & \\
\hline Other & 0.10 & \\
\hline Married & 0.49 & 0.50 \\
\hline Stroke & 0.0328 & \\
\hline Hip fracture & 0.0277 & \\
\hline Alzheimer's/dementia & 0.0510 & \\
\hline \multicolumn{3}{|l|}{$\begin{array}{l}\text { Length of stay by discharge } \\
\text { Destination }\end{array}$} \\
\hline Home & 8.00 days & 11.92 \\
\hline Long stay & 26.28 days & 37.67 \\
\hline Transfer & 14.18 days & 21.21 \\
\hline Other & 13.05 days & 25.79 \\
\hline
\end{tabular}

\subsubsection{Length of stay and inpatient bed days}

Figure 7.1 presents the cumulative inpatient bed days by LOS of the patient sample.

Patients in the 90th LOS quantile have an average LOS of 49.9 days and use 47.6 
per cent of all inpatient bed days in a given year. This figure highlights that examining the longest stays using UQR may be most appropriate to understand how LTRC bed supply impacts LOS for the small proportion of inpatients who use a disproportionate amount of inpatient care.

FIGURE 7.1 CUMULATIVE INPATIENT BED DAYS BY LENGTH OF STAY, EMERGENCY INPATIENT DISCHARGES AGED 65+, 2012-2015

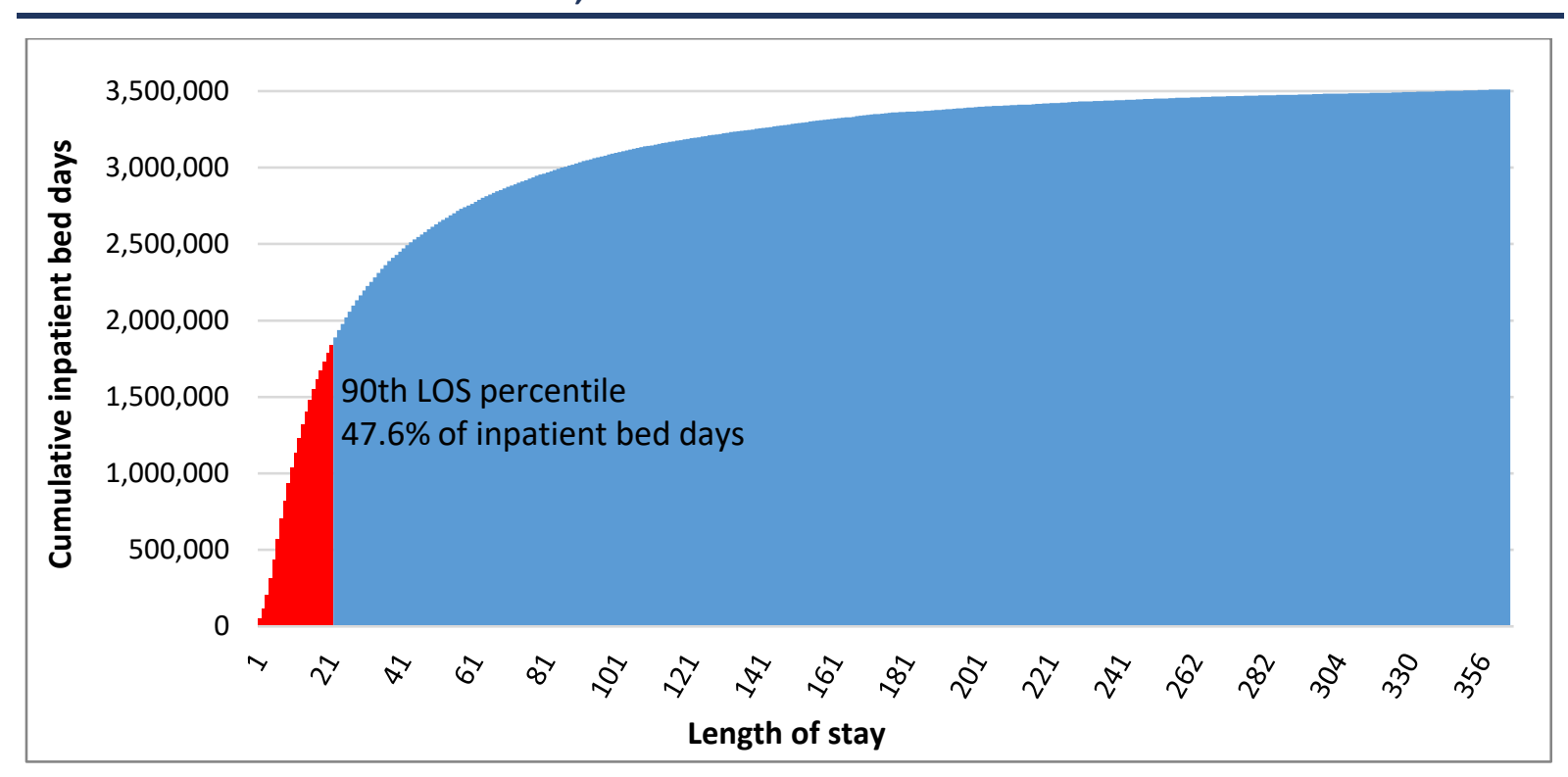

\subsubsection{Regression analyses}

Table 7.2 presents results from the linear and negative binomial regressions for the full sample. Results present the marginal effect of LTRC bed supply per capita on average LOS. Across each model, a negative and statistically significant relationship between LTRC bed supply and LOS is observed. For the 65+ sample, elasticities of 0.13 to 0.21 are observed; a 10 per cent increase in LTRC bed supply per capita is associated with a 1.3 per cent ( 0.1 days) and 2.2 per cent ( 0.2 days) reduction in average LOS. When LTRC supply is divided into quintiles there is some evidence of an inverted U-shaped relationship, with LOS lowest for patients with the lowest and the highest supply of LTRC beds. However, there are no statistically significant differences between the lowest quintile group and the others. LTRC bed elasticities are shown to increase in the $75+$ and $85+$ samples. Within the $85+$ sample, an elasticity of 0.23 is observed; a 10 per cent increase in LTRC bed supply is associated with a 2.3 per cent ( 0.3 days) reduction in LOS.

In all of these models, we control for home care supply. In all models, the elasticity on home care supply is negative and statistically significant, with elasticities similar to those observed in the previous chapter. This confirms the expectation that both home care and LTRC may substitute for inpatient LOS amongst older people. 


\begin{tabular}{|c|c|c|c|c|c|}
\hline & \multicolumn{3}{|c|}{$65+$} & $75+$ & $85+$ \\
\hline & \multicolumn{2}{|c|}{ Ordinary least squares } & \multirow{2}{*}{$\begin{array}{l}\text { Negative binomial } \\
\text { (LOS) }\end{array}$} & \multicolumn{2}{|c|}{ Ordinary least squares } \\
\hline & (Ln LOS) & (Ln LOS) & & (Ln LOS) & (Ln LOS) \\
\hline & (I) & (II) & (III) & (IV) & (V) \\
\hline Year trend & 0.006 & 0.006 & 0.005 & 0.005 & -0.002 \\
\hline Married & $-0.115^{* * *}$ & $-0.115^{* * *}$ & $-0.149 * * *$ & $-0.105^{* * *}$ & $-0.057 * * *$ \\
\hline Medical card & $0.073 * * *$ & $0.073 * * *$ & $0.065^{* * *}$ & $0.058 * *$ & $0.050^{*}$ \\
\hline Weighted Charlson score & $0.098 * * *$ & $0.098 * * *$ & $0.106^{* * *}$ & $0.109 * * *$ & $0.122 * * *$ \\
\hline Female & $0.020 * * *$ & $0.020 * * *$ & 0.006 & $0.022 * * *$ & $0.039 * * *$ \\
\hline Ln home care & $-0.126^{* *}$ & $-0.104 *$ & $-0.215^{* * *}$ & $-0.149 *$ & -0.109 \\
\hline Ln LTRC beds & $-0.130 * *$ & & $-0.210 * * *$ & $-0.151 * *$ & $-0.230 * * *$ \\
\hline \multicolumn{6}{|l|}{ LTRC beds quintiles } \\
\hline Quintile 1 (lowest) & & Base & & & \\
\hline Quintile 2 & & 0.015 & & & \\
\hline Quintile 3 & & 0.006 & & & \\
\hline Quintile 4 & & -0.018 & & & \\
\hline Quintile 5 (highest) & & -0.033 & & & \\
\hline Hospital Fes & $\mathbf{Y}$ & $\mathbf{Y}$ & $\mathbf{Y}$ & $\mathbf{Y}$ & $\mathbf{Y}$ \\
\hline Observations & 333,928 & 333,928 & 333,928 & 202,587 & 67,092 \\
\hline Clusters (hospitals) & 26 & 26 & 26 & 26 & 26 \\
\hline Adjusted R Squared & 0.272 & 0.272 & - & 0.241 & 0.210 \\
\hline Average length of stay & 10.51 days & 10.51 days & 10.51 days & 11.87 days & 13.62 days \\
\hline
\end{tabular}

Notes: $\quad$ Other covariates in models: DRG, hospital-level standardised bed capacity, age and age squared, season. Sample includes patients admitted from home through an ED, not transferred to another hospital at discharge, alive at discharge. Clustered standard errors at hospital level. 
Table 7.3 presents results from the linear and negative binomial regressions for those patients who are ultimately discharged to a long-stay facility. Results again show the marginal effect of LTRC bed supply per capita on average LOS. Across each model, a negative and statistically significant relationship between LTRC bed supply and LOS is observed, with elasticities larger than in the full sample included in Table 7.2 above; in other words, LTRC bed supply has a stronger association with LOS for those discharged to a long-stay facility. This is of greater consequence, as overall inpatient LOS for those patients discharged to a long-stay facility is almost three times as long as those discharged home, ceteris paribus.

For the $65+$ sample, elasticities of 0.33 to 0.39 are observed; a 10 per cent higher home care supply is associated with a 3.3 per cent ( 0.85 days) and 3.9 per cent ( 1 day) lower LOS.

When included as quintiles, however, again there are hints of an inverted U-shaped relationship, with LOS lowest for patients with the lowest and the highest supply of LTRC beds. Again, however, the differences between individual quintile coefficients and the lowest category are not statistically significant. LTRC bed elasticities are similar or slightly smaller in the $75+$ and $85+$ samples. 
TABLE 7.3 DETERMINANTS OF LENGTH OF STAY FOR EMERGENCY INPATIENT DISCHARGES AGED 65+DISCHARGED TO LONG-STAY FACILITY, 2012-2015

\begin{tabular}{|c|c|c|c|c|c|}
\hline & \multicolumn{3}{|c|}{$65+$} & $75+$ & $85+$ \\
\hline & \multicolumn{2}{|c|}{ Ordinary least squares } & \multirow{2}{*}{$\begin{array}{l}\text { Negative binomial } \\
\text { (LOS) }\end{array}$} & \multicolumn{2}{|c|}{ Ordinary least squares } \\
\hline & (Ln LOS) & (Ln LOS) & & (Ln LOS) & (Ln LOS) \\
\hline & (I) & (II) & (III) & (IV) & (V) \\
\hline Year trend & -0.017 & -0.013 & -0.032 & -0.020 & -0.030 \\
\hline Married & 0.019 & 0.020 & 0.001 & $0.034 * * *$ & $0.056 * * *$ \\
\hline Medical card & $0.039 * *$ & $0.039 * *$ & 0.023 & $0.041 * *$ & $0.042 *$ \\
\hline Weighted Charlson score & $0.091 * * *$ & $0.091 * * *$ & $0.089 * * *$ & $0.098 * * *$ & $0.103^{* * *}$ \\
\hline Female & $-0.036 * * *$ & $-0.036 * * *$ & $-0.046 * * *$ & $-0.033 * * *$ & -0.016 \\
\hline Home care & $-0.231 * *$ & $-0.159 *$ & $-0.323 * * *$ & $-0.276 * *$ & $-0.238^{*}$ \\
\hline Ln LTRC beds & $-0.333 * *$ & & $-0.394 * * *$ & $-0.276 * *$ & -0.303 \\
\hline \multicolumn{6}{|l|}{ LTRC beds quintiles } \\
\hline Quintile 1 (lowest) & & Base & & & \\
\hline Quintile 2 & & 0.028 & & & \\
\hline Quintile 3 & & 0.087 & & & \\
\hline Quintile 4 & & 0.017 & & & \\
\hline Quintile 5 (highest) & & -0.045 & & & \\
\hline Clusters (hospitals) & 26 & 26 & 26 & 26 & 15,315 \\
\hline Observations & 38,125 & 38,125 & 38,125 & 31,677 & 15,315 \\
\hline Adjusted R Squared & 0.209 & 0.209 & - & 0.206 & 0.197 \\
\hline Average length of stay & 25.88 days & 25.88 days & 25.88 days & 25.69 days & 24.92 days \\
\hline
\end{tabular}

Notes: $\quad$ Other covariates in models: DRG, hospital-level standardised bed capacity, age and age squared, season.

Sample includes patients admitted from home through an ED, transferred to long-stay facility at discharge, alive at discharge.

Clustered standard errors at hospital level. 
As with home care supply, LTRC bed supply may also have differing associations with LOS across patients with different characteristics. Therefore, in Figure 7.2 we interact LTRC bed supply with sex, marital status, public/private status and weighted Charlson comorbidity score to test whether increased LTRC bed supply may reduce LOS at a greater rate for females (versus males), married patients or sicker patients with more comorbidities. Results are based upon the linear regression model from Column I in Table 7.2.

Overall, we find little heterogeneity in the effect of LTRC bed supply on inpatient LOS. LTRC supply has slightly stronger effect sizes for females (versus males) and public (versus private) patients, but no statistically significant differences are observed. However, LTRC bed supply does seem to be associated with lower LOS among sicker inpatients, as measured by higher weighted Charlson comorbidity scores. This is in line with the similar results found for home care supply in the previous chapter. 
FIGURE 7.2 DETERMINANTS OF LENGTH OF STAY FOR EMERGENCY INPATIENT DISCHARGES AGED 65+ INTERACTION PLOTS, 2012-2015

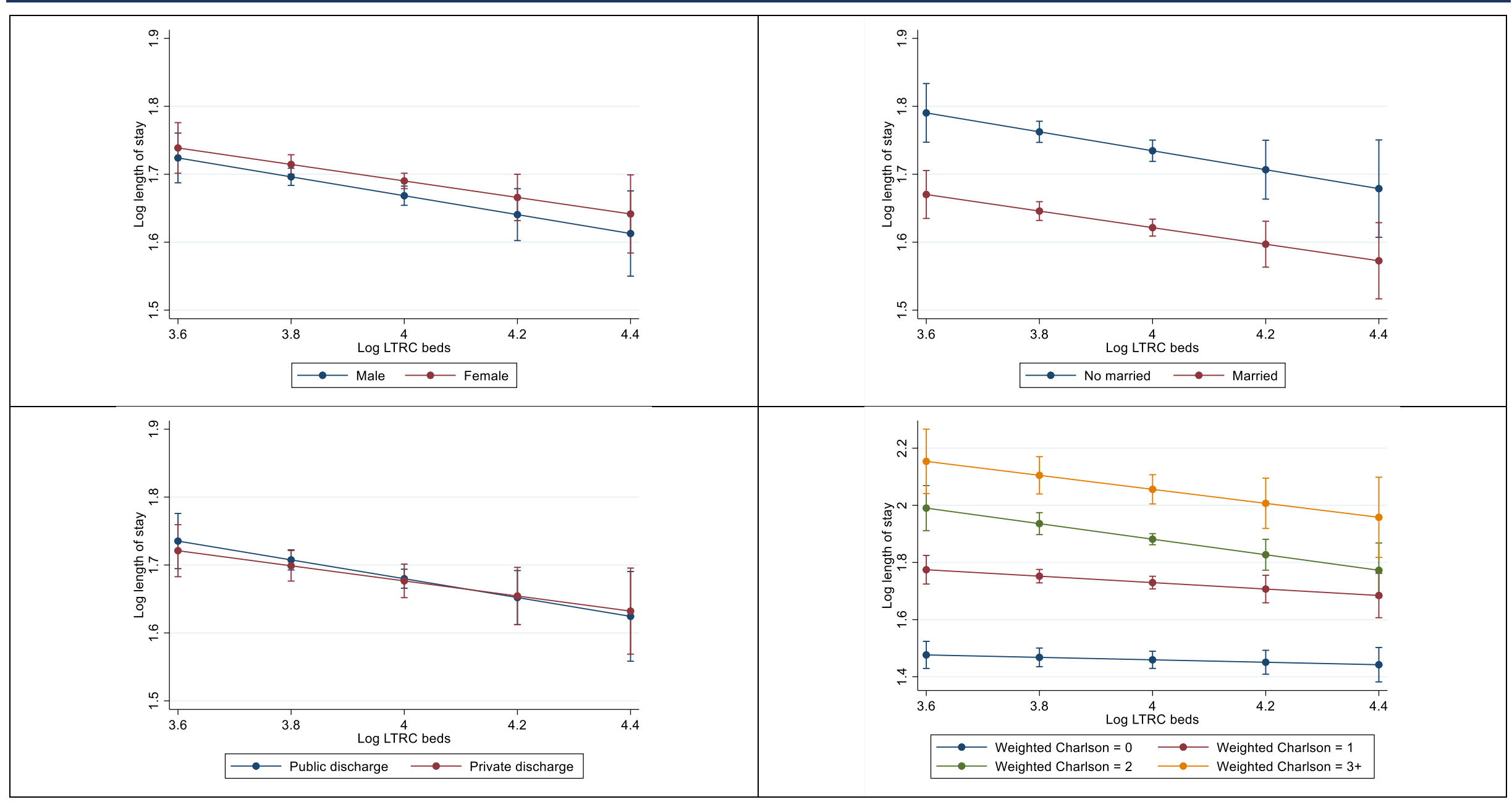

Notes: $\quad$ Ordinary least squares regressions based on Table 7.2, Column I. Covariates in models: year, married, medical card, weighted Charlson score, hospital fees, DRG, hospital-level standardised bed capacity, home care supply, age and age squared, season.

Sample: Patients admitted from home through an ED, not transferred to another hospital at discharge, alive at discharge.

Clustered standard errors at hospital level. 
The results presented in Table 7.2 and Table 7.3 show that LTRC is associated with lower LOS on average. In Figure 7.3 and Figure 7.4, we present results from the UQR where the impact of LTRC supply is examined across LOS quantiles, for all emergency inpatient discharges aged 65 years and over, and those ultimately discharged to an LTRC centre.

In Figure 7.3, examining all emergency inpatients aged 65 years and over, LTRC bed supply has a stronger negative association with reducing LOS for patients with longer LOS. While a small, negative coefficient is observed for an LOS of 4 days and above, the coefficients are larger for those with an LOS greater - in the 90th (LOS of greater than 22 days) and 95th quantiles (LOS of greater than 35 days). Transforming the coefficients to be interpreted as elasticities, 10 per cent higher LTRC bed supply is associated with a 0.5 days lower LOS for those in the 90th percentile (equating to a 2.2 per cent LOS reduction) and 1.2 days lower LOS for those in the 95th percentile (equating to 3.3 per cent LOS reduction). In this regard, higher LTRC bed supply seems to be associated with a greater reduction in LOS in both marginal and absolute terms for patients who may be characterised as delayed discharges.

FIGURE 7.3 UNCONDITIONAL QUANTILE REGRESSION, EMERGENCY INPATIENT DISCHARGES FOR PATIENTS AGED 65+, 2012-2015

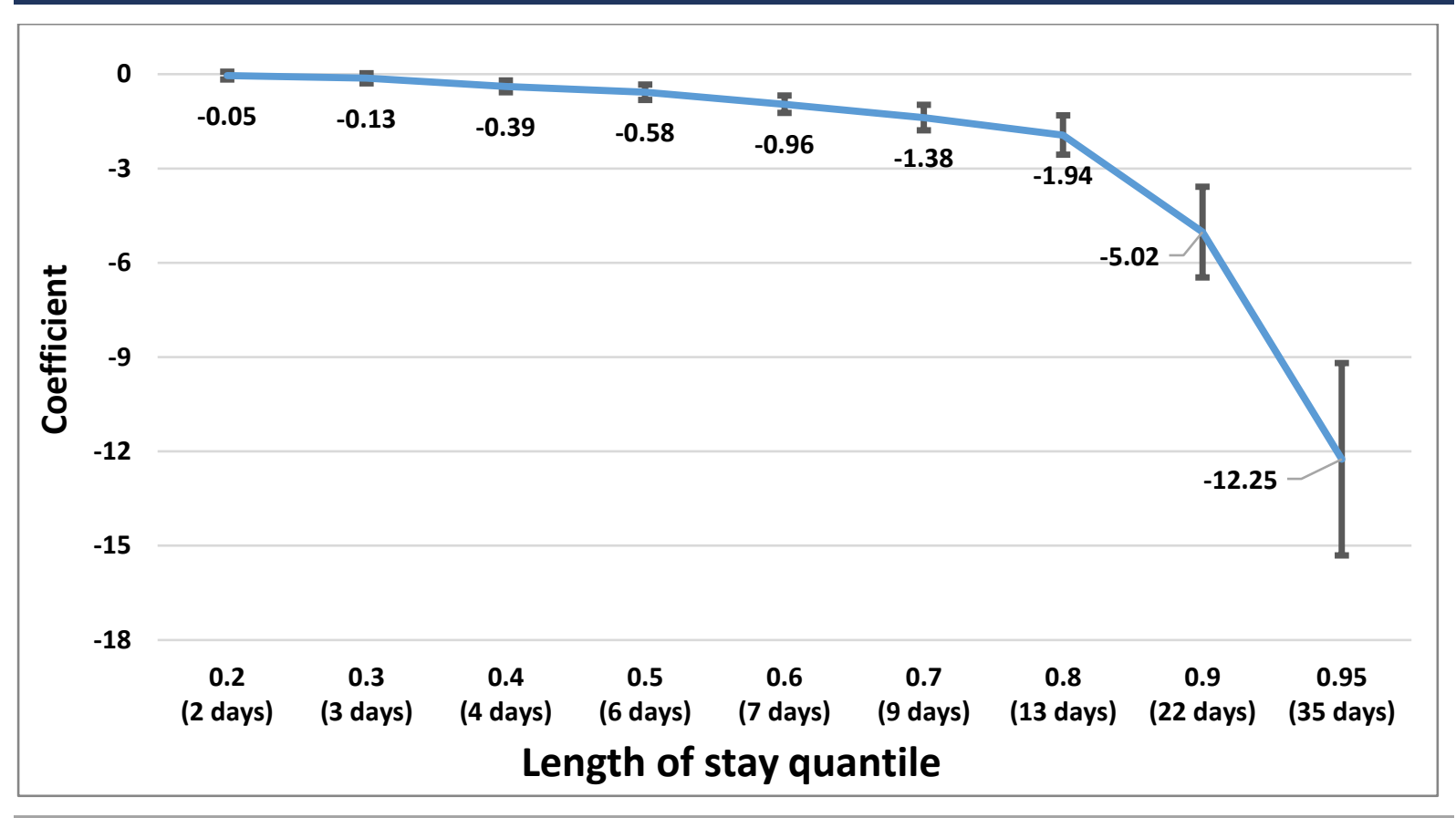

In Figure 7.4, examining all those inpatient discharged to an LTRC facility, LTRC bed supply once more has a stronger negative association with LOS among patients with longer LOS. The size of the coefficients is also much larger than that observed in the previous graph. Transforming the coefficients to be interpreted as elasticities, 10 per cent higher LTRC bed supply is associated with a 3.3 days lower 
LOS for those in the 90th percentile (equating to 5.3 per cent LOS reduction) and a 6.8 days lower LOS for those in the 95th percentile (equating to 7 per cent LOS reduction).

It is clear that the relationship between LTRC bed supply and LOS is much stronger for those ultimately discharged to an LTRC centre, especially for those with longer LOS, who are more likely to be affected by delayed discharges.

FIGURE 7.4 EMERGENCY INPATIENT DISCHARGES DISCHARGED TO LONG-STAY FACILITY FOR PATIENTS AGED 65+, 2012-2015

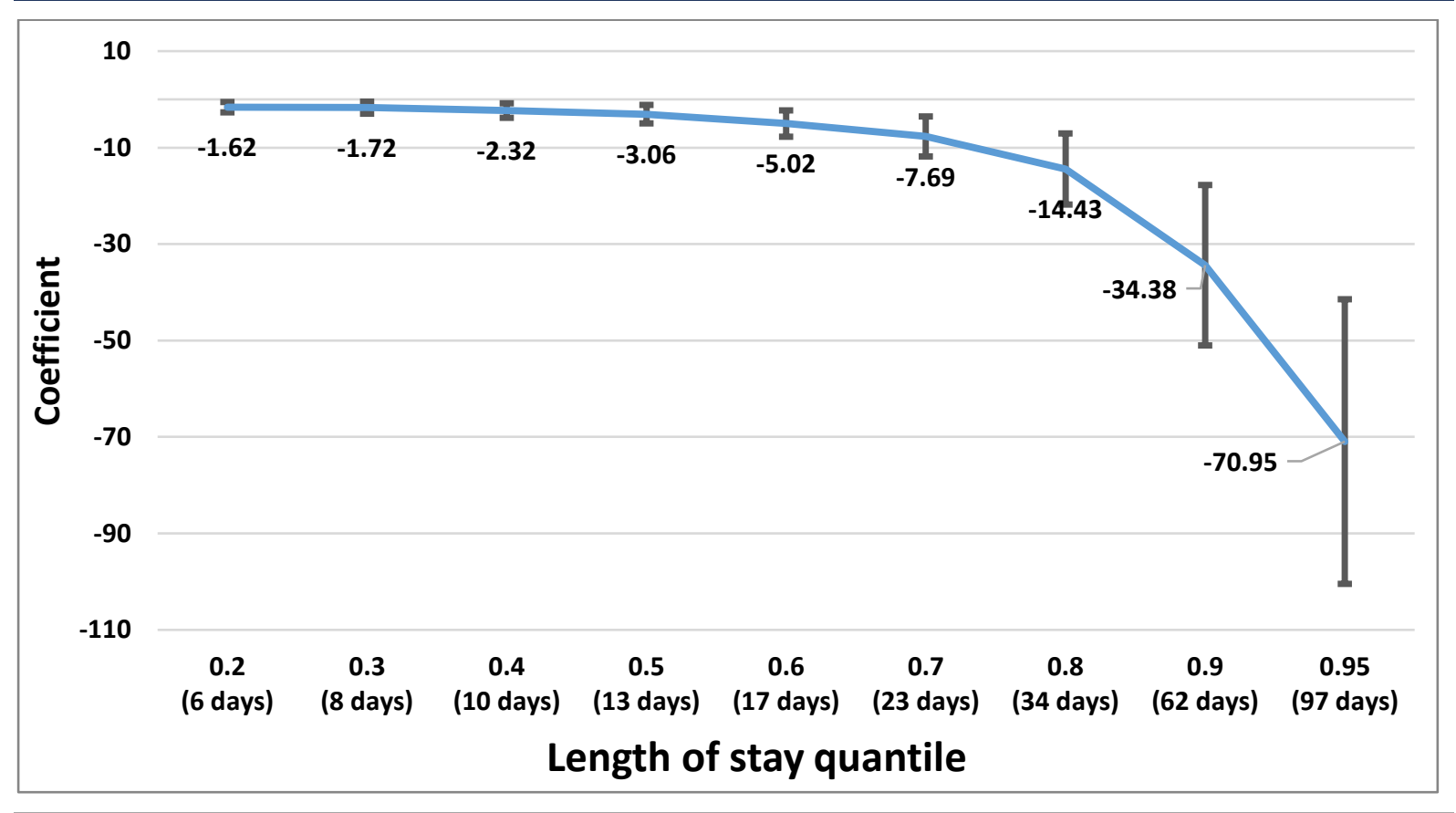

Stroke, hip fracture, and Alzheimer's/dementia patients

Stroke and hip fracture patients may be more amenable to LTRC, while it is known that a clear correlate of LTRC use is cognitive impairment conditions, specifically Alzheimer's disease and dementia. Table 7.4, Table 7.5 and Table 7.6 present results from the linear and negative binomial regressions examining the impact of LTRC bed supply on LOS for these patient groups. Due to smaller numbers, it was not possible to undertake UQRs for these patient groups. As shown in Table 7.4, we found no statistically significant relationship between LTRC bed and LOS for stroke patients, though the sign and scale of the coefficients are similar to those in the tables above. 
TABLE 7.4 DETERMINANTS OF LENGTH OF STAY FOR EMERGENCY INPATIENT STROKE DISCHARGES AGED 65+, 2012-2015

\begin{tabular}{|c|c|c|c|c|c|}
\hline & \multicolumn{3}{|r|}{$\cos$} & $75+$ & $85+$ \\
\hline & \multicolumn{2}{|c|}{ Ordinary least squares } & \multirow{2}{*}{$\begin{array}{l}\text { Negative binomial } \\
\text { (LOS) }\end{array}$} & \multicolumn{2}{|c|}{ Ordinary least squares } \\
\hline & (Ln LOS) & (Ln LOS) & & (Ln LOS) & (Ln LOS) \\
\hline & (I) & (II) & (III) & (IV) & (V) \\
\hline Year trend & -0.010 & -0.011 & -0.017 & -0.008 & -0.009 \\
\hline Married & $-0.140 * * *$ & $-0.139 * * *$ & $-0.168 * * *$ & $-0.131 * * *$ & $-0.076 * * *$ \\
\hline Medical card & 0.038 & 0.039 & 0.033 & 0.026 & -0.015 \\
\hline Weighted Charlson score & $0.070 * * *$ & $0.070 * * *$ & $0.056 * * *$ & $0.056 * * *$ & 0.020 \\
\hline Female & -0.023 & -0.023 & -0.038 & -0.006 & -0.056 \\
\hline Ln home care & $-0.234 * * *$ & $-0.201 * *$ & $-0.304 * * *$ & $-0.291 * * *$ & $-0.256^{*}$ \\
\hline \multicolumn{6}{|l|}{ LTRC beds } \\
\hline Ln LTRC beds & -0.199 & & -0.275 & -0.167 & -0.095 \\
\hline \multicolumn{6}{|l|}{ LTRC beds quintiles } \\
\hline Quintile 1 (lowest) & & Base & & & \\
\hline Quintile 2 & & -0.004 & & & \\
\hline Quintile 3 & & -0.001 & & & \\
\hline Quintile 4 & & -0.055 & & & \\
\hline Quintile 5 (highest) & & -0.056 & & & \\
\hline Clusters (hospitals) & 26 & 26 & 26 & 26 & 26 \\
\hline Observations & 10,775 & 10,775 & 10,775 & 7,015 & 2,406 \\
\hline Adjusted R Squared & 0.232 & 0.232 & & 0.229 & 0.214 \\
\hline Average length of stay & 24.22 days & 24.22 days & 24.22 days & 26.68 days & 29.43 days \\
\hline
\end{tabular}

Notes: Other covariates in models: hospital-level standardised bed capacity, age and age squared, season, stroke severity.

Sample: Patients with primary or secondary stroke diagnosis, admitted from home through an ED, not transferred to another hospital at discharge, alive at discharge. Clustered standard errors at hospital level. 
Table 7.5 presents results from linear and negative binomial regressions for hip fracture patients. A negative and statistically significant relationship between LTRC bed supply and LOS is observed. Within the 65 years and over group, 10 per cent higher LTRC supply is associated with a 2.5 per cent ( 0.51 days) lower LOS for hip fracture patients. When the supply variable is included in quintile form, it loses statistical significance as it did in the full-population model. The strength of the relationship between LTRC bed supply and hip fracture LOS does not increases with age. 
TABLE 7.5 DETERMINANTS OF LENGTH OF STAY FOR EMERGENCY INPATIENT HIP FRACTURE DISCHARGES AGED 65+, 2012-2015

\begin{tabular}{|c|c|c|c|c|c|}
\hline & \multicolumn{3}{|c|}{$65+$} & $75+$ & \multirow{2}{*}{ ares $85+$} \\
\hline & \multicolumn{2}{|c|}{ Ordinary least squares } & Negative binomial & Ordinary least squares & \\
\hline & (Ln LOS) & (Ln LOS) & (LOS) & (Ln LOS) & (Ln LOS) \\
\hline & (I) & (II) & (III) & (IV) & (V) \\
\hline Year trend & -0.016 & -0.010 & -0.012 & -0.020 & -0.023 \\
\hline Married & $-0.069 * * *$ & $-0.068 * * *$ & $-0.118^{* * *}$ & $-0.041^{* *}$ & -0.021 \\
\hline Medical card & $0.116^{* * *}$ & $0.117^{* * *}$ & $0.071 * * *$ & $0.117 * * *$ & $0.093 * *$ \\
\hline Weighted Charlson score & $0.153^{* * *}$ & $0.153^{* * *}$ & $0.193 * * *$ & $0.156^{* * *}$ & $0.153^{* * *}$ \\
\hline Female & $-0.090 * * *$ & $-0.089 * * *$ & $-0.139 * * *$ & $-0.059 * *$ & -0.017 \\
\hline Ln home care & -0.086 & -0.069 & $-0.140^{*}$ & -0.046 & -0.176 \\
\hline \multicolumn{6}{|l|}{ LTRC beds } \\
\hline Ln LTRC beds & $-0.250 * *$ & & $-0.294 * * *$ & $-0.234^{*}$ & -0.197 \\
\hline \multicolumn{6}{|l|}{ LTRC beds quintiles } \\
\hline Quintile 1 (lowest) & & Base & & & \\
\hline Quintile 2 & & -0.038 & & & \\
\hline Quintile 3 & & -0.009 & & & \\
\hline Quintile 4 & & -0.010 & & & \\
\hline Quintile 5 (highest) & & -0.063 & & & \\
\hline Clusters (hospitals) & 26 & 26 & 26 & 26 & 26 \\
\hline Observations & 9,264 & 9,264 & 9,264 & 7,244 & 3,414 \\
\hline Adjusted R Squared & 0.267 & 0.266 & & 0.254 & 0.247 \\
\hline Average length of stay & 20.42 days & 20.42 days & 20.42 days & 21.68 days & 23.44 days \\
\hline
\end{tabular}

Notes: $\quad$ Other covariates in models: hospital-level standardised bed capacity, age and age squared, season.

Sample: Patients with primary or secondary hip fracture diagnosis, admitted from home through an ED, not transferred to another hospital at discharge, alive at discharge.

Clustered standard errors at hospital level. 
Table 7.6 presents results from linear and negative binomial regressions for Alzheimer's disease and dementia patients. Overall, similar to results for stroke patients, the table shows a non-statistically significant relationship between LTRC bed supply and LOS for those aged 65 years and over. However, within the 85 years and over sample, there is a clear negative and statistically significant relationship; a 10 per cent higher LTRC supply is associated with 5 per cent (1.17 days) lower LOS for Alzheimer's disease/dementia patients. This finding, and the insignificant effect of home care, may be a consequence of older patients with Alzheimer's disease or dementia being more amenable to LTRC and younger patients with these conditions being more amenable to home care. 


\begin{tabular}{|c|c|c|c|c|c|}
\hline & \multicolumn{3}{|c|}{$65+$} & $75+$ & \multirow{2}{*}{ es $85+$} \\
\hline & \multicolumn{2}{|c|}{ Ordinary least squares } & Negative binomial & Ordinary least squares & \\
\hline & (Ln LOS) & (Ln LOS) & (LOS) & (Ln LOS) & (Ln LOS) \\
\hline & (I) & (II) & (III) & (IV) & $(\mathrm{V})$ \\
\hline Year trend & 0.015 & 0.015 & 0.011 & 0.009 & -0.031 \\
\hline Married & $-0.077 * * *$ & $-0.077^{* * *}$ & -0.085 & $-0.078 * * *$ & 0.002 \\
\hline Medical card & 0.025 & 0.025 & -0.016 & 0.032 & 0.061 \\
\hline Weighted Charlson score & $0.092 * * *$ & $0.092 * * *$ & $0.065 * * *$ & $0.087 * * *$ & $0.063 * * *$ \\
\hline Female & $-0.055^{* * *}$ & $-0.055^{* * *}$ & $-0.088 * * *$ & $-0.0546 * * *$ & $-0.054 * * *$ \\
\hline Ln home care & $-0.253^{* * *}$ & $-0.253^{* * *}$ & $-0.416 * * *$ & -0.281 & -0.010 \\
\hline \multicolumn{6}{|l|}{ LTRC beds } \\
\hline Ln LTRC beds & -0.027 & & -0.191 & -0.102 & $-0.501 * *$ \\
\hline \multicolumn{6}{|l|}{ LTRC beds quintiles } \\
\hline Quintile 1 (lowest) & & Base & & & \\
\hline Quintile 2 & & 0.050 & & & \\
\hline Quintile 3 & & 0.051 & & & \\
\hline Quintile 4 & & 0.065 & & & \\
\hline Quintile 5 (highest) & & 0.012 & & & \\
\hline Clusters (hospitals) & 26 & 26 & 26 & 26 & 26 \\
\hline Observations & 17,037 & 17,037 & 17,037 & 14,765 & 6,755 \\
\hline Adjusted R Squared & 0.267 & 0.266 & & 0.254 & 0.247 \\
\hline Average length of stay & 23.90 days & 23.90 days & 23.90 days & 23.850 days & 23.46 days \\
\hline
\end{tabular}

Notes: Other covariates in models: hospital-level standardised bed capacity, age and age squared, season.

Sample: patients with primary or secondary hip fracture diagnosis, admitted from home through an ED, not transferred to another hospital at discharge, alive at discharge. Clustered standard errors at hospital level. 


\subsubsection{Placebo tests}

In this chapter, we use differences in the supply of LTRC across areas and over time, controlling for a range of patient-level characteristics, to estimate the relationship between LTRC and timing of discharge for older emergency inpatients admitted from home. In this context, the expected substitution effect relates to those who are potentially new LTRC residents. We do not include those admitted as inpatients from long-stay facilities as we assume that these patients will return to their previous LTRC bed once they are medically fit to do so.

Areas with better LTRC supply may also have better non-acute and social care facilities more generally, and LTRC may be acting as a proxy for better non-acute service supply in an area, or for other unobserved factors that impact LOS. Therefore, we test whether LTRC bed supply impacts patients admitted from longstay facilities.

Table 7.7 presents results from the linear regression analysis of the determinants of LOS for emergency inpatients aged 65 years and over admitted from a long-stay facility. Coefficients on marital status, weighted Charlson score medical card status and sex are consistent with those observed in Table 7.2. However, no statistically significant coefficient on the LTRC bed supply is observed. Interestingly, the coefficient on home care is also small and insignificant, which again makes sense as we would not expect home care to impact the LOS of patients admitted as an inpatient from a long-stay facility. Results from the placebo analyses suggest that the negative relationship observed between LTRC supply and inpatient LOS in inpatients aged 65 years and over, who were admitted from home, is unlikely to be driven by an underlying, omitted, non-acute care variable. 
TABLE 7.7 DETERMINANTS OF LENGTH OF STAY FOR EMERGENCY INPATIENT DISCHARGES AGED 65+ ADMITTED FROM A LONG-STAY FACILITY, 2012-2015

\begin{tabular}{|l|c|}
\hline & $\begin{array}{c}\text { Ordinary least squares } \\
\text { (Ln LOS) }\end{array}$ \\
\hline Year trend & $(\mathrm{I})$ \\
\hline Married & 0.014 \\
\hline Medical card & $-0.030^{* *}$ \\
\hline Weighted Charlson score & 0.004 \\
\hline Female & $0.074^{* * *}$ \\
\hline Ln home care hours & -0.004 \\
\hline LTRC & -0.050 \\
\hline Ln LTRC beds & \\
\hline Clusters (hospitals) & -0.060 \\
\hline Observations & 26 \\
\hline Adjusted R Squared & 21,223 \\
\hline
\end{tabular}

Notes: Linear regression analyses. Other covariates in models: DRG, hospital-level standardised bed capacity, age and age squared, season.

Sample: patients admitted from home through an ED, not transferred to another hospital at discharge, alive at discharge.

Clustered standard errors at hospital level for Ireland sample.

\subsubsection{Probability of discharge to long-stay centre}

While this is not the focus of this chapter, we also examine the impact of home care supply on the probability of patients being discharged to a long-stay centre.

Table 7.8 presents results from the linear regression analysis of the determinants of discharge to a long-stay centre for emergency inpatients aged 65 years and over.

Overall, we find a negative statistically significant association between home care supply and discharge to a long-stay centre for emergency inpatients aged 65 years and over. A 10 per cent increase in home care supply reduces the probability of discharge to a long-stay facility by 3.7 percentage points. Including home care supply in quintiles, patients in areas in the highest quintile of home care supply have a probability of being discharged to an LTRC centre 3 percentage points lower than patients in areas in the lowest quintile of home care supply. This result is a large effect as the underlying rate of discharge to a long-stay facility in the sample is 10.7 per cent. These results show that while much of the attention is on substitution of acute care with non-acute care for older people in this report, an important area of future analysis will be to examine how home care and LTRC care also substitute. 
TABLE 7.8 PROBABILITY OF DISCHARGE TO A LONG-STAY CENTRE FOR EMERGENCY INPATIENT DISCHARGES AGED 65+, 2012-2015

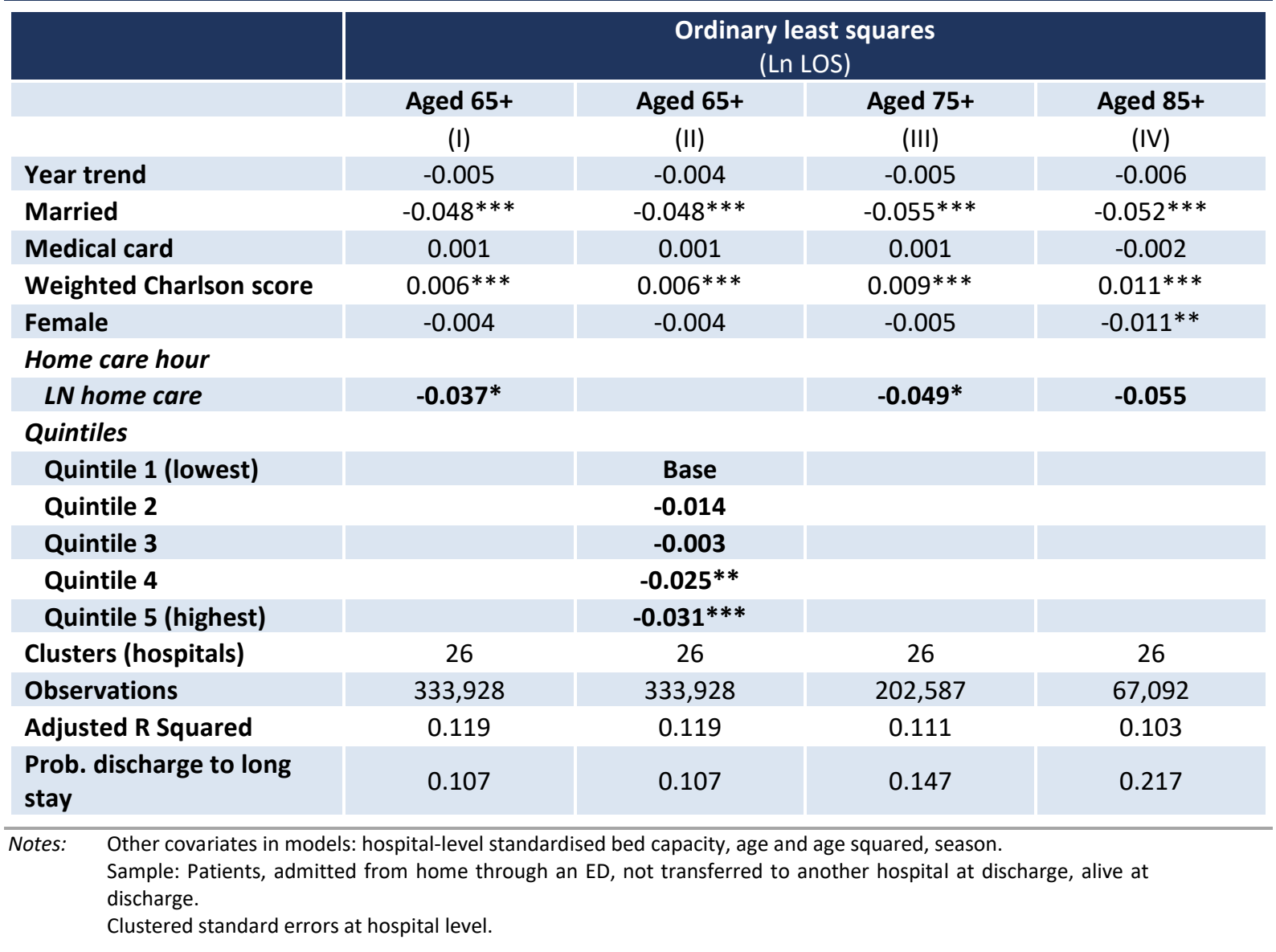

\subsection{CONCLUSIONS}

Findings from this chapter show that LTRC bed supply is associated with reductions in inpatient LOS for older people admitted from home. However, similar to the previous chapter's findings, the size of the substitution effect differs significantly across models and patient samples.

When average LOS across all emergency inpatients admitted from home who are aged 65 years and over is examined, both linear and negative binomial regressions show a substitution effect, with elasticities of 0.13-0.22 estimated. Ceteris paribus, a 10 per cent higher LTRC bed supply per capita is associated with an approximate 1.3-2.2 per cent reduction in average inpatient LOS. As another way of illustrating the scale of the effects, we can express the impact of LTRC beds per capita in terms of inpatient bed days. In these data, average LOS is 10.51 days, and 3.51 million inpatient bed days were used between 2012 and 2015. Reducing LOS by up to 2.2 per cent ( 0.2 days) equates to 19,000 fewer inpatient beds per annum.

When we examine those patients who ultimately are discharged to an LTRC centre, and are therefore inherently more amenable to LTRC supply, a stronger substitution effect is found. In this sample, a 10 per cent higher LTRC bed supply 
home care supply is associated with a 3.3-3.9 per cent reduction in average inpatient LOS. As another way of illustrating the scale of the effects, we can express the impact of LTRC beds per capita in terms of inpatient bed days. In these data, average LOS is 25.88 days, and 0.987 million inpatient bed days were used between 2012 and 2015. Reducing LOS by 3.3 per cent (0.1 days) equates to 8,141 fewer inpatient beds per annum.

However, examining those with long LOS (delayed discharges), a larger substitutive effect of LTRC beds per capita is found. Results from UQR analyses show that for those in the 90th percentile of LOS, a 10 per cent increase in per capita LTRC bed supply is associated with a 0.5 days less spent in hospital. The relationship is stronger in the 95th percentile, where a 10 per cent increase in per capita LTRC bed supply is associated with a 1.2 days reduction in LOS.

Returning again to those patients who ultimately are discharged to an LTRC centre, and are therefore inherently more amenable to LTRC supply, a stronger substitution effect is found for the longest stayers, and this effect is intensified by the long LOS for this group more generally. Results from UQR analyses show that for those in the 90th percentile of LOS, a 10 per cent increase in per capita LTRC bed supply is associated with 3.3 fewer days in hospital. The relationship is stronger in the 95th percentile, where a 10 per cent increase in per capita LTRC bed supply is associated with a 6.8 days reduction in LOS.

These substitutive effects are found even after controlling for both inpatient bed supply ('push' factor) and home care supply (a potentially alternative 'pull' factor). The findings of a negative relationship between LTRC supply and average inpatient LOS is consistent with previous evidence from the NHS (Fernandez and Forder, 2008; Forder, 2009). However, while the elasticities for LTRC are larger than for home care, the differences in effect sizes are small. Furthermore, the results from the UQR analysis are similar to those found in the NHS for delayed discharges. Gaughan et al. (2015) found that that a 10 per cent increase in LTRC beds reduced delayed discharges (where need for social care is given as the reason for the discharge delay) by between 6 per and 9 per cent. In our results, we also find a clear association between LTRC bed supply per capita and reduced LOS for those with longer LOS (potentially delayed discharges).

Results for stroke, hip fracture patients and Alzheimer's/dementia patients - three groups of patients that are more likely to benefit from LTRC - do not show consistent negative substitution effects. However, for Alzheimer's disease and dementia patients aged 85 years and over, a large negative relationship is seen between LOS and LTRC supply. The coefficients, while large, are often statistically insignificant at the most meaningful levels, which is largely a result of smaller sample size and clustering of standard errors. 
Examining the homogeneity of results across demographic groups (gender, marital status, public/private status and Charlson comorbidity illness score), little differences are observed across gender, marital status and private discharge status. However, similar to the results in home care, the substitution effects are slightly larger for sicker patients.

Caution should be taken in interpreting the results on LTRC supply as causal estimates. Our LTRC bed measure includes a range of long-stay, short-stay and rehabilitation beds, all of which may not be amenable to the inpatients in our sample. In addition, our measure of LTRC supply is based upon a patient's LHO; however, patients who ultimately end up in an LTRC centre may choose a centre outside of their LHO due to the perceived quality of the chosen centre, ability to access a bed quickly, or to be closer to family. In this context, it is uncertain whether the within-hospital differences we accounted for in the previous chapter also apply here. Additionally, we only include inpatients admitted from home. Therefore, all results should be interpreted as the impact of LTRC supply on the inpatient LOS for those not already residing in an LTRC centre. Notwithstanding these caveats, this is the first finding on the impact of LTRC supply on use of acute hospital services to date in Ireland, and in that sense marks an important contribution to our understanding of adds substitution between the two services. Furthermore, in an exploratory analysis we find that the availability of home care reduces the probability of patients who are admitted to hospital from home being discharged to LRTC; this is also new information on the interaction between services in Ireland, and worthy of further study. 


\section{CHAPTER 8}

\section{Concluding discussion and policy recommendations}

\subsection{SCOPE OF CHAPTER}

In this chapter, we discuss findings from the project and highlight its key conclusions for policy, alongside other factors that may impact resource allocation decisions for health and social care services in Ireland.

Section 8.2 describes the project, while Sections 8.3 and 8.4 provide an overview of its key findings across the two reports. Section 8.5 discusses these findings in the context of resource allocation in Ireland and draws on other key factors that may impact resource allocation decisions. Section 8.6 concludes.

\subsection{INTRODUCTION}

This report is part of a broader project entitled, An inter-sectoral analysis by geographic area of the need for and the supply and utilisation of health services in Ireland. The project's analyses were undertaken in the context of a healthcare system with significant capacity constraints within the acute hospital sector and current policy priorities to achieve a move towards providing more care, where appropriate, in the community and closer to home.

In the initial stage of the project, Smith et al. (2019) provided evidence about the supply of non-acute care across counties in Ireland, to help identify whether adequate capacity exists to meet increased (or even existing) demand, should more care be transferred to non-acute services. The analysis undertaken in this report provides evidence on the way in which acute and non-acute sectors interact in the system, especially the substitution effects between inpatient care and care of older people services and home care and long-term residential care (LTRC).

\subsection{GEOGRAPHIC PROFILE OF HEALTHCARE NEEDS AND NON-ACUTE HEALTHCARE SUPPLY IN IRELAND (SMITH ET AL., 2019)}

Smith et al. (2019) provided evidence on the geographic distribution of non-acute care across Ireland. That analyses relied on per capita supply estimates to allow for accurate comparisons across areas. It also examined whether supply differed when proxies for healthcare need and eligibility were accounted for. The breadth of the services examined was wide, with 10 non-acute services - which constitute the vast majority of non-acute care in Ireland - examined (Wren et al., 2017). These were: general practitioners (GPs); community nurses; physiotherapists; occupational therapists; speech and language therapists; podiatrists and chiropodists; counsellors and psychologists; social workers; LTRC beds and home 
care hours. Due to data limitations, for many services only public supply could be analysed. However, across GPs (who are private providers), physiotherapists and LTRC beds, private supply data were available.

Large geographic inequalities were observed across all services, with the largest geographic inequalities in supply seen for podiatrists and chiropodists, driven largely by many areas having no non-acute podiatrist or chiropodist. Gini coefficients of greater than 0.1 , often used as a rule of thumb as the measure of large inequalities, were estimated for all services apart from GPs, who have a Gini of 0.096. This means that for all services examined, a redistribution of over 10 per cent of supply from areas in the upper half of the distribution to areas in the lower half of the distribution would be required to achieve perfect equality of supply (van Doorslaer and Koolman, 2004).

However, importantly in healthcare, analysis of relative supply should also take relative need into account. Therefore, a key feature of the analyses was the adjustment of supply for a range of eligibility and need factors. Eligibility was based on medical card and GP visit card rates, and need factors followed from those included in the Andersen's Behavioural Model of Health Services Use. However, the adjustment factors had little impact and the geographic distribution of each non-acute service remains unequal.

While Smith et al. (2019) provide clear evidence that the geographic distribution of non-acute care supply is unequal, there also appears to be notable consistency across the supply types in terms of the areas that have low levels of per capita supply relative to the national value. Figure 8.1 provides a summary overview of Smith et al. (2019) findings on the geographic variation in the per capita supply of non-acute and long-term care services. Counties with supply of a service 10 per cent lower than the national average are denoted by a red circle. Counties with supply of a service 10 per cent higher than the national average are denoted by a green circle. Where per capita supply of a service is within 10 per cent of the national average, this is denoted by an amber circle. Counties are sorted by the number of services, where supply is lower than the national average. The greater Dublin commuter belt and south east counties have lower relative supply of many non-acute primary and community care services than the national average. Kildare and Meath have lower relative supply (at least 10 per cent lower than the national average) for all non-acute community and primary care services. Wexford and Wicklow have lower relative supply (at least 10 per cent lower than the national average) for 7 of the eight non-acute community and primary care services examined.

In contrast to the low relative supply on the east coast, three counties on the west coast - Galway, Sligo and Leitrim - as well as Cork, Westmeath and Tipperary South 
have higher relative supply for many services, and do not have lower relative supply for more than one of the services examined.

In general in Dublin, the supply of services in Dublin North and Dublin South is similar to the national average. Some variation in supply is seen across some services. Dublin North has low relative supply of GPs, counsellors and psychologists and LTRC. Dublin South has low relative supply of counsellors and psychologists, and publicly funded home care hours.

Smith et al. (2019) do not analyse the reasons for these patterns of supply but the findings of low supply in the commuter belt counties with rapidly growing population around Dublin is consistent with earlier studies and suggestive that health service planning does not respond adequately to population patterns (Brick et al., 2010; Layte et al., 2009). 
FIGURE 8.1 SUMMARY OF RELATIVE SERVICE SUPPLY INDICATORS IN IRELAND, 2014

\begin{tabular}{|c|c|c|c|c|c|c|c|c|c|c|c|}
\hline & \multicolumn{8}{|c|}{ Non-Acute Primary and Community Care } & \multicolumn{2}{|c|}{ Long-Term Care } & \multirow{2}{*}{$\begin{array}{l}\text { Number of services } \\
>10 \text { per cent below } \\
\text { national average }\end{array}$} \\
\hline & $G P^{1}$ & $\mathrm{PHN} / \mathrm{CN}^{2}$ & $\mathrm{PT}^{3}$ & $\mathrm{oT}^{2}$ & $\mathrm{SLT}^{2}$ & $P \& C^{2}$ & CO\&PSY ${ }^{2}$ & $s w^{2}$ & LTRC $^{4}$ & $\mathrm{HCH}^{5}$ & \\
\hline Kildare & 0 & O & 0 & 0 & 0 & 0 & 0 & 0 & 0 & 0 & 9 \\
\hline Meath & 0 & 0 & 0 & 0 & 0 & 0 & 0 & 0 & 0 & 0 & 8 \\
\hline Wexford & 0 & 0 & 0 & 0 & 0 & 0 & 0 & 0 & 0 & 0 & 8 \\
\hline Wicklow & 0 & 0 & O & 0 & 0 & O & 0 & 0 & O & O & 8 \\
\hline Clare & 0 & 0 & 0 & 0 & 0 & 0 & 0 & 0 & 0 & 0 & 7 \\
\hline Kilkenny & 0 & 0 & 0 & 0 & 0 & 0 & 0 & 0 & 0 & 0 & 7 \\
\hline Waterford & 0 & 0 & 0 & 0 & 0 & $\mathrm{O}$ & 0 & 0 & 0 & 0 & 7 \\
\hline Offaly & 0 & 0 & 0 & 0 & 0 & 0 & 0 & 0 & 0 & 0 & 5 \\
\hline Carlow & 0 & 0 & 0 & 0 & 0 & 0 & O & 0 & 0 & 0 & 5 \\
\hline Laois & 0 & 0 & 0 & 0 & 0 & 0 & 0 & 0 & O & 0 & 4 \\
\hline Limerick & 0 & 0 & 0 & 0 & 0 & 0 & 0 & 0 & 0 & 0 & 3 \\
\hline Mayo & 0 & 0 & 0 & 0 & 0 & 0 & 0 & 0 & 0 & 0 & 3 \\
\hline Tipperary North & 0 & 0 & 0 & 0 & 0 & 0 & 0 & 0 & 0 & 0 & 3 \\
\hline Dublin North & 0 & 0 & 0 & 0 & 0 & 0 & 0 & 0 & 0 & 0 & 3 \\
\hline Kerry & 0 & 0 & 0 & 0 & 0 & 0 & 0 & 0 & 0 & 0 & 3 \\
\hline Monaghan & 0 & 0 & 0 & 0 & 0 & 0 & 0 & 0 & 0 & 0 & 3 \\
\hline Longford & 0 & 0 & 0 & 0 & 0 & 0 & 0 & 0 & 0 & 0 & 3 \\
\hline Roscommon & 0 & O & 0 & 0 & O & 0 & 0 & 0 & 0 & 0 & 3 \\
\hline Dublin South & 0 & 0 & 0 & 0 & 0 & ? & 0 & 0 & 0 & 0 & 2 \\
\hline Cavan & 0 & 0 & 0 & 0 & O & O & 0 & 0 & 0 & 0 & 2 \\
\hline Louth & 0 & 0 & 0 & 0 & 0 & 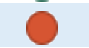 & 0 & 0 & 0 & 0 & 2 \\
\hline Donegal & 0 & 0 & 0 & 0 & 0 & O & 0 & 0 & 0 & 0 & 2 \\
\hline Tipperary South & 0 & 0 & 0 & 0 & 0 & O & 0 & 0 & 0 & 0 & 1 \\
\hline Westmeath & 0 & 0 & 0 & 0 & 0 & 0 & 0 & 0 & 0 & 0 & 1 \\
\hline Leitrim & 0 & 0 & 0 & 0 & 0 & 0 & 0 & 0 & 0 & ค & 1 \\
\hline Sligo & 0 & 0 & 0 & 0 & 0 & 0 & 0 & 0 & 0 & 0 & 1 \\
\hline Cork & 0 & 0 & 0 & 0 & 0 & 0 & 0 & 0 & 0 & 0 & 0 \\
\hline Galway & 0 & 0 & 0 & 0 & 0 & 0 & 0 & 0 & 0 & 0 & 0 \\
\hline
\end{tabular}

County has supply at least 10 per cent higher than national average.

County has supply at least 10 per cent lower than national average.

County has supply approximately equal to the national average.

Source: Smith et al. (2019).

Notes: $\quad 1$. The number of GPs is converted to estimated whole-time equivalents (WTEs), based on survey evidence on fulland part-time working practices of GPs in Ireland. See Chapter 3 for more details. 2. Publicly employed WTEs. See Chapter 3 for more details. 3. Publicly employed and privately employed WTE PTs. See Chapter 3 for more details. 4. LTRC beds in public and private LTRC centres (supply of beds per 1,000 population aged $65+$ ). See Chapter 3 for more details. 5. Publicly funded home care hours from the home help and home care package schemes (supply of hours per population aged $65+$ ). See Chapter 3 for more details.

\subsection{ANALYSIS OF THE EFFECTS ON IRISH HOSPITAL CARE OF THE SUPPLY OF CARE INSIDE AND OUTSIDE THE HOSPITAL (WALSH ET AL., 2019)}

This report analyses supply in the acute hospital, home care and LTRC sectors, three sectors that provide a substantial amount of the country's health and social care (Wren et al., 2017). It finds that access to and supply of these services differ across areas, and that variations are evident over time. The report models the impact of 'push' (inpatient bed supply) and 'pull' (home care and LTRC supply) 
factors on hospital care in Ireland, using measures of inpatient bed supply, home care supply per capita, and LTRC bed supply in the analyses.

Chapter 3 shows that the acute public hospital system in Ireland has seen significant reconfiguration of services in recent years. There has been a consolidation of emergency department (ED) services in the public hospital system, with many hospitals having their ED services reduced from '24/7 365' status (24 hours a day, 7 days a week). This has resulted in some local hospitals losing fulltime ED services. During the economic recession from 2008, there was a dramatic reduction in inpatient bed supply and staffing levels in the public hospital system. Inpatient bed supply fell by 13 per cent between 2007 and 2012, together with a similar reduction in doctor and nursing WTEs. This was followed by a slight increase in supply from 2013 to 2015 . These changes in bed supply were driven by economic stress and related pressures on government finances rather than lower demand for health services.

Table 8.1 highlights the main results from chapters 5-7 of this report. In relation to the impact of inpatient bed supply on emergency inpatient length of stay (LOS) Chapter 5 - in recent years, inpatient bed supply decreases and subsequent increases follow a similar trajectory to inpatient LOS (reductions in supply associated with reduced LOS and vice versa). Using econometric modelling techniques that exploit differences in bed supply across hospitals and within hospitals over time, we find that a large proportion of the LOS changes observed in recent years are explained by changes in inpatient bed supply. These results imply that a 40 per cent of inpatient LOS reductions between 2010 and 2012 were a result of reduced numbers of inpatient beds.

While Chapter 5 highlights the role of acute bed supply on inpatient LOS, Chapters 6 and 7 examine the impact of non-acute care for older people on LOS, using granular data on per capita publicly financed home care supply and per capita LTRC bed supply in public and private LTRC centres. However, the size of the substitution effect differs significantly across models, whether average LOS or delayed discharges is examined, and across groups of patients who differ by diagnosis.

Chapter 6 illustrates the substitution effect between home care and inpatient LOS. Findings from this chapter show that when average LOS across all emergency inpatients admitted from home aged 65 years and over are examined, ceteris paribus, increased home care supply has a statistically significant negative impact on LOS. We express results in terms of a 10 per cent increase in home care supply per capita (similar to the increased observed in Ireland between 2012 and 2015), equating to 1.5 million additional home care hours in 2015. This 10 per cent increase implies an approximate 1 per cent reduction in average inpatient LOS for inpatients aged 65 years and over. Illustrated in terms of inpatient bed days, 1.5 
million additional home care hours are associated with a reduction of 14,700 inpatient bed days in 2015. If results from the Dublin North analyses are imposed on the Irish sample, owing to the substantial increase in home care supply in Dublin North between 2012 and 2015, ceteris paribus, a 10 per cent increase in home care supply per capita is associated with an approximate 2.7 per cent reduction in average LOS, and 1.5 million additional home care hours implies a reduction of 40,000 inpatient bed days per annum and freeing up 110 beds daily. ${ }^{46}$ Examining the effect of home care supply on those with long LOS (delayed discharges), results from unconditional quantile regression (UQR) analyses show that for those in the 90th percentile of LOS (those with LOS of 21 days or more) a 10 per cent increase in per capita home care supply is associated with a 0.3 days less spent in hospital. Similarly, if results from the Dublin North UQR analysis were imposed countrywide, then even larger reductions, or 2 days, would be estimated.

Chapter 7 illustrates the substitution effect between LTRC bed supply and inpatient LOS. Findings from this chapter show that when average LOS across all emergency inpatients admitted from home who are aged 65 years and over is examined, ceteris paribus, increased LTRC bed supply is found to have a statistically significant negative impact on LOS. We express results in terms of a 10 per cent increase in LTRC bed supply per capita, which equates to 2,965 LTRC beds in 2015. This 10 per cent increase in LTRC bed supply per capita is associated with an approximate 1.32.2 per cent reduction in average inpatient LOS. Illustrated in terms of inpatient bed days, an addition of 2,965 beds in LTRC centres is associated with a reduction of 19,000 inpatient bed days per annum using the upper bound elasticity, or 53 available inpatient beds daily. Examining those emergency inpatients aged 65 years and over ultimately discharged to a long-stay facility, a 10 per cent increase in LTRC care supply per capita is associated with an approximate 3.3-3.9 per cent reduction in average inpatient LOS. Illustrated in terms of inpatient bed days, the addition of 2,965 beds in LTRC centres is associated with a reduction of 9,720 inpatient bed days per annum using the upper bound elasticity, for those patients who end up in an LTRC centre. In 2015, emergency inpatients admitted from home and discharged to an LTRC centre used approximately 250,000 inpatient bed days. Therefore, the 10 per increase in LTRC be supply would reduce this amount by 4 per cent for this group of inpatients. Examining the effect of LTRC bed supply on those with long LOS (delayed discharges), results from UQR analyses show that for those in the 90th percentile of LOS (those with LOS of 22 days or more), a 10 per cent increase in per capita home care supply is associated with 0.5 days less spent in hospital. Furthermore, for those ultimately discharged to a long-stay facility, results from UQR analyses show that for those in the 90th percentile of LOS (those

46 In 2015, there were 1.44 million emergency inpatient bed days in Irish public hospitals used by patients aged 65 years and over. 
with LOS of 62 days or more), a 10 per cent increase in per capita home care supply is associated with 3.3 fewer days in hospital.

TABLE 8.1 EFFECTS OF HOME CARE AND LTRC SUPPLY ON EMERGENCY INPATIENT DISCHARGES AGED 65+ IN RELATION TO LENGTH OF STAY AND DELAYED DISCHARGES

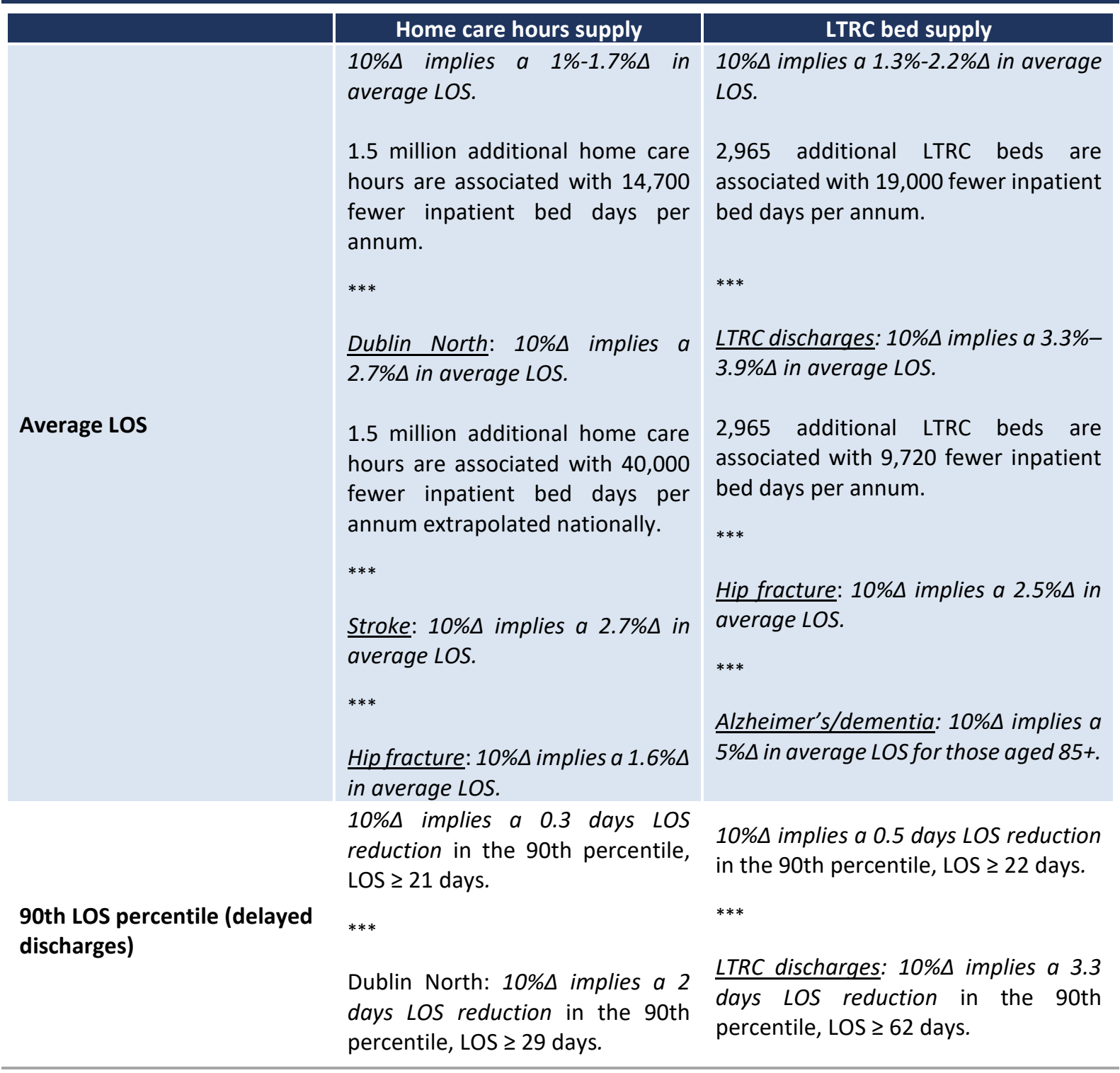

Notes: $\mathrm{SD}=$ standard deviation; $\Delta=$ change.

The results in Chapter 5 on the clear, but often ignored, link between beds and hospitalisation use have broad implications for policymakers in both Ireland and internationally. From an Irish perspective, it is clear that there was, and is, an inadequate inpatient bed supply in public hospitals in Ireland. This can be assessed by a number of outcomes, including the long waiting times for elective care (Siciliani et al., 2014; Wren et al., 2017) and the fact that Ireland has the highest bed occupancy rate in the OECD (OECD, 2018). The Government now acknowledges that a greater number of beds are required (Government of Ireland, 2018a; Keegan et al., 2018a). However, the recent reduction in bed capacity was also followed by sharp reductions in staffing levels. This makes the experience in Ireland different to other countries, such as Denmark (Christiansen and Vrangbæk, 
2017), that implemented bed capacity reductions as a result of system change, and where increases in staffing often accompanied bed reductions.

There are examples where, at the onset of an economic crisis, governments initially enact a counter-cyclical policy by expanding healthcare expenditure in response to recession (Keegan et al., 2013). In Ireland, initial counter-cyclical policies were subsequently replaced by severe cuts to expenditure. Previous analyses of the Irish healthcare system during the economic crisis have argued that efficiencies were seen in the public hospital system at the beginning of the recessionary period (2008-2012), with hospitals 'doing more with less', 'more' being reflected in inpatient and day patient activity and 'less' in reduced budgets (Burke et al., 2014). However, if 'doing more' leads to unsafe occupancy rates, this is of concern, and the authors suggest that the continued lack of staffing resources and capacity resulted in lower activity and increased waiting lists post-2012 (Burke et al., 2014).

From an international perspective, the findings in Chapter 5 also highlight why one should be cautious when using LOS as a measure of efficiency. LOS can be a useful measure of efficiency when comparing hospitals and health systems. However, interpretations of LOS reductions are clearly dependent upon the context in which they are observed. In periods where expenditure cuts or system changes occur, lower LOS may reflect efficiency gains, but may also simply be indicative of the lack of resources or available bed capacity, as opposed to reduced demand for care. Other countries such as the UK and Canada also have occupancy rates higher than the 85 per cent recommended threshold. In the English NHS for instance, cuts to acute bed numbers in recent years have increased occupancy rates from 87 per cent in 2010-2011 to over 90 per cent in 2016-2017 (The Kings Fund, 2017). Therefore, in those countries with high occupancy rates, using LOS as a measure of efficiency may require caution, especially as shorter inpatient LOS often results in greater readmission rates (Ambugo and Hagen, 2019; Carey, 2015; Martin et al., 2016) and high occupancy has been shown to cause large negative effects in patient outcomes (Boden et al., 2015; Madsen et al., 2014; Schilling et al., 2010). As explained in this report, the lack of an individual health identifier (IHI) makes it difficult to examine mortality and readmissions as outcomes in Ireland. However, given that Ireland has the highest inpatient bed occupancy rates in the EU, these latter findings in the broader literature are worrying, and worthy of further research.

Private hospitals provide approximately 15 per cent of all inpatient care in Ireland (Wren et al., 2017), with the majority of this care being elective or less complex emergency care. Due to the lack of information on private hospital care, it is difficult to ascertain the impact of bed capacity changes on elective LOS in public hospitals. This suggests that in order to understand the impact of non-acute care supply on elective care in public hospitals, information on private hospitals would also be required. 
Results on the substitution effects of home care and LTRC are pertinent to both Irish and international policymakers. The analyses show that the impact of longterm care on inpatient LOS depends upon how the relationship is measured. When measured in terms of average LOS, results are broadly in line with previous studies on the substitution of long-term care (Costa-Font et al., 2018; Fernandez and Forder, 2008; Forder et al., 2018; Gonçalves and Weaver, 2017). However, this is not the case regarding the effect of home care in terms of reducing average LOS, which in Ireland is as large, or larger, than the effect of LTRC supply. Examining both home care and LTRC together, an increase in supply of both by 10 per cent (1.5 million home care hours and 2,965 LTRC beds) implies a reduction of over 34,000 inpatient bed days, or freeing up over 90 inpatient beds daily. This equates to 2.3 per cent of all inpatient beds days used by those aged 65 years and over per annum, including those who may not be suitable for either home care or LTRC. ${ }^{47}$ However, for delayed discharges results are stronger, and follow previous results from the NHS (Gaughan et al., 2015), with increases in long-term care having larger reductions in LOS for delayed discharges.

These findings show that substitution effects exist and that large increases in longterm care supply may reduce inpatient LOS, but by a disproportionately small amount. The Nuffield Trust recently stated:

[W] need to beware a narrative that fixing social care will fix the problems of the NHS. It won't, but it would help. Secondly, it cannot be assumed that alternatives to hospital will save large amounts of money unless far more radical changes to the system are made.

(Edwards, 2017)

This quote is apt to these findings and the Irish health system: while home care in particular is vital for older patients, the increases required may not have substantial effects on reducing hospital care requirements for older people. However, there is evidence in this project that policymakers may see the most sizable impact by targeting delayed discharges.

The findings in Chapter 6 and Chapter 7 emphasise the extent to which factors influencing inpatient LOS are often outside the control of hospital management. Policies that penalise hospitals for longer LOS, such as those included in many activity-based funding ( $A B F$ ) initiatives (for instance, remunerating them only for the expected LOS for a particular procedure/condition) may exacerbate the challenges they face, due to inadequate resourcing of care outside the hospital setting. Unlike many other countries, both health and social care in Ireland are the responsibility of one organisation: the HSE. Therefore, in theory at least, this

47 In 2015, there were 1.44 million emergency inpatient bed days in Irish public hospitals used by patients aged 65 years and over. 
provides a more viable avenue in which to coordinate health and social care thereby facilitating, for example, earlier discharge home from hospital - and to tie remuneration to efficiencies and performance, both within and outside hospital.

As the Fair Deal scheme is a statutory scheme for LTRC, administered by the HSE, greater coordination of inpatient and LTRC services, within each LHO, may afford greater reductions in long inpatient stays and delayed discharges. Furthermore, in many cases if the patient awaits Fair Deal funding to access to their centre of choice, the use of intermediate-stay facilities may free-up scarce inpatient beds.

Ireland is unique in a European context, in the sense that the age-dependency ratio is currently more favourable than that found in most European countries. However, as the population continues to grow, the ageing of the population in coming decades will be acute (Wren et al., 2017). Between 2015 and 2030, the population aged 65 years and over is projected to increase by approximately 60 per cent, resulting in projected demand for long-term care increasing by at least 40 per cent (Wren et al., 2017). Therefore, decisions are required regarding investment in and planning of home care and LTRC care services.

\subsection{POLICY RECOMMENDATIONS FOR RESOURCE ALLOCATION}

The findings from this project outline the importance of developing an efficient resource allocative decision making process in the Ireland health system. Plans are now in place to increase capital funding of the health system, and acute and nonacute capacity within the system in coming years (Government of Ireland, 2018a). However, accompanying increases in current expenditure are also required (staff to tend the additional capacity), though there is much less information available on medium-term current expenditure plans. Capacity and supply increases also require a plan that relates distribution to area need to maximise the potential benefits to the population. It is therefore welcome that Strategic Action 3.1 of the Sláintecare Implementation Strategy states a commitment to developing a plan for the health service based on population health planning and regional budget allocations to guide detailed service planning in 2019 (Department of Health, 2019).

In relation to such envisaged reforms, we discuss some potential barriers to efficient allocation decisions and, where appropriate, draw on findings from this project to make specific recommendations for policymakers. Many of the recommendations build on ideas put forward previously, such as in the 2010 ESRI report, Resource allocation and financing in the health sector (Brick et al., 2010) and in the report, Towards the development of a resource allocation model for primary, continuing and community care in the health services (Vega et al., 2010). We expand on these reports, drawing from on our project findings and in the context of recent changes to health and social care in Ireland. 
All health systems face the challenge of how best to allocate funding and resources to allow for an efficient, equitable and affordable health and social care system. As a previous report on resource allocation in Ireland acknowledged, 'there is no single correct way to allocate resources, and there is no perfect model', and differences in models used are seen across countries (Vega et al., 2010). In Ireland, there has been a lack of systematic resource allocation decision-making, which is unique for a system that is largely tax-funded. As outlined in Smith et al. (2019), while there have been a number of policy changes and proposals in recent years, many initiatives failed to attract sustained political support and some were not fully implemented after receiving more in-depth scrutiny (for example, universal health insurance). There is little doubt that this lack of a long-term systematic allocative decision process has hindered health outcomes and has not optimised improvement in health outcomes or efficiency in health expenditure. However, guidelines set out within Sláintecare do provide overarching commitments to invest in a regional-based long-term systematic allocative decision process. This cross-party political support for a more long-term plan for the future direction of the public health system in Ireland represents an opportunity to improve planning and resource allocation (Department of Health, 2018a; Government of Ireland, 2018a; Houses of the Oireachtas Committee on the Future of Healthcare, 2017). In the context of such health policy developments, we discuss five important factors that should be addressed to allow for efficient and transparent resource allocation decision making.

\subsubsection{Improved health system data}

In order to make informed decisions about how best to allocate resources, gathering appropriate data and carrying out necessary research are vital. However, Ireland is a laggard with respect to consistent and comprehensive data collection throughout all sectors of the health and social care system. This project has highlighted how lack of granular health data is impeding the formulation of wellfounded policy choices and the research that can be undertaken on the Irish health system. Previous policy analyses, including by the Public Service Pay Commission, has also commented on the significant data limitations to inform recent analyses on recruitment and retention in the health service (Public Service Pay Commission, 2018). This lack of data prevails particularly in the community sector, where it has been highlighted as a barrier to efficient resource allocation in Ireland in the past (Brick et al., 2010) and will continue to do so in the short term, as we discuss below. We believe this project has demonstrated how data improvements, regarding the supply of acute and non-acute care specifically, can expand the knowledge base for both researchers and policymakers.

This project has, for the first time in Ireland, detailed the supply of the 10 most prominent non-acute services across areas. The project captured data from a range of different sources - a time-consuming and research intensive exercise. A number of gaps in the data were identified, in particular on the supply of private health 
professionals, GP practice nurses and non-acute care utilisation. The lack of information on private non-acute care supply affects not only understanding of these services, but also understanding of resource allocation and workforce planning for the public health system.

The main challenges arising from the lack of information on private healthcare utilisation and supply are:

- determining overall demand for non-acute care, presently and in the future;

- determining provision of care supply across areas and identifying those areas where increased public and private supply may be required;

- analysing the effects of any eligibility changes in the context of Sláintecare recommendations for universal healthcare, which could make hitherto privately accessed services available or financed through the public system; and

- formulating plans to train the future health workforce.

The last point (on training the future health workforce) should be understood in the context that both the public and private sectors are 'fishing from the same pool' of potential workers; therefore, policies required to attract workers into the public health system depend upon information on the private sector. Furthermore, in the Irish health system, private providers are contracted to deliver a number of non-acute services, arrangements that are not captured in available data, with the most prominent example being GPs providing care to General Medical Services (GMS) patients. A large proportion of publicly financed home care is provided by voluntary and for-profit organisations and there may even be overlap of public and private care; publicly employed public health nurses, publicly employed occupational therapists and for-profit home care workers all provide care to a patient as part of a home support package. In the LTRC sector, over two-thirds of beds provided to Fair Deal scheme residents are provided by private centres, while in the past, in order to alleviate waiting lists, private speech and language therapists were contracted for care. The ratios of public-to-private staff across professions is also likely to vary by profession; for example, physiotherapy (Eighan et al., 2019), Again, further information in required on how services are organised at the local level.

The issue of lack of information on private care supply and utilisation in non-acute services is mirrored in the acute hospital sector. There is no definitive information on any of the following aspects of acute private hospital care in Ireland:

- number of ED attendances;

- number of outpatient attendances;

- number of day patient visits;

- number of inpatients stays;

- number of average LOS per inpatient stay; 
- average bed occupancy;

- number of day patient and inpatient beds;

- patient casemix;

- nurse and medical doctors (including consultants) WTEs; or

- number of public patients (on the National Treatment Purchase Fund) provided with care.

While there has been recent analysis on some of the above (Keegan et al., 2018a; Wren et al., 2017), this lack of information hinders our understanding of the nature of the role played by private hospitals in the provision of acute care. It also curtails the research that can be conducted on the public hospital system. As detailed in Chapter 4 of this report, the option for individuals, especially those with private health insurance, to choose between public and private hospital provision of elective day patient and elective inpatient care makes it difficult to analyse measures such as elective inpatient LOS in public hospitals over time. It is possible that any changes observed in public hospital LOS for elective inpatients could simply reflect a changing composition of groups attending the public system, which could be driven by unobserved changes in private hospital care rather than factors within public hospitals.

New data sources that are becoming available could be used for future analysis of community care and may aid improvement in resource allocation. CORU is a new multi-profession regulator responsible for the registration of health and social care professionals, with registration open for physiotherapists, occupational therapists and speech and language therapists. ${ }^{48}$ The CORU database will capture all personnel who wish to practice as an allied health professional in Ireland. However, in these administrative databases collected by organisations such as CORU and the Medical Council, it would be advisable for the scope to expand by capturing factors such as public and private provision and WTEs. This additional information would be invaluable to researchers and policymakers. Where a number of new or existing data sources capture similar information, the ability to reconcile these data is also important. In this project, for instance, we estimate the number of GPs across geographical areas using data and methods developed over time and used in a number of previous studies (Mohan et al., 2019; Teljeur et al., 2010; Teljeur et al., 2014). However, other estimates of GP numbers and GP WTEs are also published (Medical Council, 2019). The multiplicity of different sources of information can result in confusion for policymakers and the public.

Efficient resource allocation decisions require a large amount of data on population health, population trends, health and social care demand and utilisation and care supply and workforce provision. To aid equitable allocation of services in relation to need across areas, this information is required at a granular demographic and 
regional level. The implementation of the $\mathrm{IHI}$ and electronic health records throughout the system has the potential to revolutionise research and evidencebased decision making in Ireland. Therefore, a key long-term goal of all health planning and resources polices should be incremental improvements in the data infrastructure, across all areas, on a continuing basis.

\subsubsection{Better understanding of substitution and mechanisms to achieve integration of care}

Three of the most important and influential planning documents on the Irish health and social care system in recent years are: the Sláintecare implementation strategy, the Department of Health's Health service capacity review, and the National development plan (Department of Health, 2018a; Government of Ireland, 2018a; Houses of the Oireachtas Committee on the Future of Healthcare, 2017). These documents, in particular the Sláintecare implementation strategy (Government of Ireland, 2018b), have set out views on the future organisation and integration of health and social care systems. These plans inevitably make significant assumptions about both substitution towards non-acute care and greater integration of acute and non-acute health and social care in Ireland.

One of the 'strategic actions' of the Sláintecare implementation strategy involves the expansion of community-based care in Ireland. Bringing care closer to home, where appropriate and feasible, will require increases in workforces providing primary care, community care and long-term care services. Expansion of such services should reduce some of the pressure on the acute system. In this report, we have shown how capacity in acute care settings and the supply of non-acute will both impact upon acute public hospital use. However, it is important that planning is based on evidence about the potential effects of substitution across care sectors. Unrealistic assumptions about the ability to substitute care into the community may lead to further inadequate resourcing of the acute system and a continuation of low inpatient bed numbers, high occupancy rates and poor waiting times in EDs and for elective care.

Results from Chapter 6 and Chapter 7 show that lower emergency inpatient LOS does occur in areas with better home care and LTRC provision. It is clear from this analysis however that increasing non-acute care is not a panacea for the health system, and unrealistic assumptions could lead to inefficient resource allocation. Our results clearly show that for those groups whose care is most amenable to long-term care services, such as patients with stroke, hip fracture patients and those who experience delayed discharges, better local provision of non-acute care reduces inpatient LOS. In the case of home care, where we are more confident that our results represent causal effects, a 10 per cent increase in per capita supply (equivalent to 1.5 million extra hours nationally in 2015) implies freeing up over 14,700 inpatient bed days per annum, which is the equivalent of adding 40 beds in 
the system. However, results suggest that the substitution effects are complicated, with those with the longest LOS seeing the greatest impact of home care supply, and areas such as Dublin North, which had large increases in home care, seeing larger reductions in LOS. Imposing results from Dublin North countrywide, a 10 per cent increase in home care implies freeing up over 40,000 inpatient bed days per annum, which is the equivalent of adding 110 beds in the system, and 2 days fewer spent in hospital for delayed discharges. Achieving this result required a substantial investment in home care, with Dublin North seeing home care hours increase from an estimated 1.465 million home care hours in 2012 to 2.321 million home care hours in 2015. Results on LTRC supply also show that better access to an LTRC bed is associated with lower LOS, especially for those experiencing a delayed discharge who are discharged to an LTRC centre.

It is an important caveat of this report's findings on home care that they do not reflect the impact any future home care arrangements will have on hospital use. As part of Sláintecare, a new statutory home care scheme is scheduled to be established in 2021. While few details of this scheme are available, there is a strong likelihood that a statutory assessment tool (SAT) will be used to assess need for home care; however, we are unsure whether some form of co-payment may be required. Future work will be required to model the impacts of the new home care scheme on use of hospital services. Currently, there may be perverse incentives to seek residential care over home care; for example, there is no statutory scheme regarding eligibility for or access to home care, geographical inequities exist regarding the public supply, and there is substantial private purchase. By contrast, an equitably applied system largely involving state funding is in place for LTRC, albeit one that also involves geographical inequities of supply. One complication of the new home care scheme will be that its introduction will involve two statutory arrangements being in place for long-term care - Fair Deal and the new home care one - with little understanding of how these two schemes will interact and integrate. As we found in Chapter 7, home care may reduce the use of LTRC for those discharged from hospital. Therefore, work should take place to examine the benefits of greater integration of both statutory schemes, especially where individuals prefer to stay at home and where home care resourcing would allow for this to occur safely, since home care is much less costly than LRTC for those who are in a position to avail of the former.

There are other areas where substitution should be examined to improve resource allocation and reduce pressure on services. For example, demand on the GP sector will increase if more emphasis is placed upon providing care outside of hospitals, since there will be more people with illnesses and disabilities being cared for in the community. Demand for GP care will also increase with the further planned extension of free GP care to all children (Irish Medical Organisation, 2019). Providing a greater role for practice nurses in general practice can facilitate increasing supply to meet demand, subject to increased investment in practice 
nurse recruitment and expansion in the range of services delivered by nurses (Teljeur et al., 2010); this may also help to reduce the cost of GP care in Ireland (Connolly et al., 2018).

Realising the full potential benefits of substitution requires a truly integrated health and social care system, in which the continuum of care and complementarities of the preventive, primary, community and long-term care sectors are planned for in a defined, parallel and transparent manner, with incentives aligned to facilitate provision of care in the most appropriate setting for each case. An important facet of healthcare systems to allow for efficient flow of patients through a care pathway revolves around the concept of integrated care. In a 2002 report on the future of the NHS, it was highlighted that an integrated, joined-up system was required in England (Wanless, 2002). This report stated:

Although there are substitution possibilities between health and social care, simply increasing the provision of the latter without making arrangements to coordinate/integrate will be the least effective strategy.

(Wanless, 2002)

Subsequently, a crisis in NHS hospital care has been attributed to cutbacks in social care expenditure, which is funded through local government in the UK (Crawford et al., 2018). This importance of integrated care has also been highlighted as a key aspect of Sláintecare. In Ireland, unlike in many other countries, the HSE administers both the health and social care systems, which should facilitate integration of all health and social care. Furthermore, regional integrated care organisations (RICOs) will have the responsibility for overseeing both hospital services and primary, community and continuing care and thereby potentially increasing the potential of integration across services.

\subsubsection{Workforce planning}

The provision of quality and efficient healthcare services depends upon having sufficient workforce numbers at the national and regional levels, since shortages of workforce in certain sectors can greatly curtail service provision, inadvertently place pressure on other sectors in the system and adversely impact patient outcomes (Department of Health, 2017d). In order to determine allocation of resources through a health system, consideration of both short-term and longterm capacity and workforce constraints is needed. This requires a good understanding of the current workforce, the ability to recruit and retain workers and the necessary level of newly trained personnel to meet future demand requirements. We show in this report that significant regional inequalities in workforce numbers are already observed in Ireland and that workforce planning will be required to allow for more equal distribution of health and social care supply across areas. 
One of the 'strategic actions' of the Sláintecare implementation strategy focuses on expanding community-based care and increasing workforce capacity in general practice, community nursing and a range of non-acute allied health professional staff. Additional demand resulting from system reforms will add to large projected increases in health and social care demand arising from population growth and ageing across all sectors of the health system (Wren et al., 2017).

A further complication of expanding care provided outside of hospitals is that some of the largest increases in demand are projected to occur in non-acute care. We estimate the potential reduction in inpatient LOS that can be achieved by increasing home care and LTRC supply. However, even without considering substitution towards these services in the future, the workforce implications of the demand already projected are daunting. Between 2015 and 2030, even accounting for healthy ageing in the older population in the future and before addressing unmet demand, demand for home care and LTRC are projected to increase by between 40 and 54 per cent (Wren et al., 2017). As these sectors are heavily reliant on private care provision, greater integration between public and private workforce planning will be required to meet future demands.

\subsubsection{An allocation system designed to achieve equity of care supply relative to need across geographic areas}

A clear finding of this project is that there are large inequalities in non-acute care supply across areas, even adjusting for factors which may impact healthcare need, such as age, morbidity rates and deprivation, as Smith et al. (2019) also show. These findings demonstrate that in general the lack of a resource allocation methodology for health and social care services in Ireland mitigates against providing care across areas in an equitable manner. In Ireland, healthcare resources have often been distributed traditionally on the basis of historical allocations to existing providers and facilities (Brick et al., 2010).

Other healthcare systems provide exemplars of how defined resource allocation formulae have been developed and used to reduce socioeconomic and area-level inequalities. In the NHS, the Carr-Hill resource allocation formula is the basis for funding. This formula provides funding based upon the patient characteristics of each practice, such as age, sex, the proportion of patients in LTRC centres, differential patient turnover (morbidity and mortality in local area), as well as rurality of the practice and a staff market forces factor, which accounts for the geographical variation in staff costs that practices will incur (BMA, 2015). In general, the NHS policy of increasing the proportion of resources allocated to the most deprived areas has resulted in a reduction in absolute health inequalities from causes amenable to healthcare (Barr et al., 2014). Specific programmes such as Equitable Access to Primary Medical Care (EAPMC) designed to provide additional funding for new GP practices in under-served areas resulted in a relative 
increase in GP supply in deprived areas, reducing inequalities in the distribution of GP care across regions (Asaria et al., 2016).

The 2019 Sláintecare action plan proposes to develop an integrated regional resource allocation formula (Department of Health, 2019). To have a centrally administered, tax-funded healthcare system without a transparent, objective and consistent resource allocation method is unusual internationally (Brick et al., 2010; Vega et al., 2010). One area where resource allocation formulae may have the largest impact in the short term is in the area of general practice, specifically on the capitation payment system to GPs. At the moment, capitation is based upon age and sex (Connolly et al., 2018). A recent agreement was concluded with the Irish Medical Organisation, whereby many of the Financial Emergency in the Public Interest (FEMPI) expenditure cuts were reversed, and additional funds were also allocated for the management of chronic diseases and to support GP practices in the most deprived areas (Irish Medical Organisation, 2019). Policies such as these not only allow for more equitable allocation of funding but could also be important in attracting doctors to under-served areas.

\subsubsection{Regular review of resource allocation in line with regional demographic projections}

A final and often overlooked aspect of resource allocation decisions, and of particular concern in Ireland, relates to the projected increase and ageing of the population in coming decades. Healthcare system capacity pressures, especially in acute hospitals, in recent years have arisen not only from reductions in health expenditure during the recent economic recession, but also from failure to adequately plan for the increasing population. Between 1996 and 2016, the Irish population increased by over 30 per cent (Wren et al., 2017), yet for many services, such as inpatient bed supply, absolute reductions in supply occurred. As the total population in Ireland has been projected to increase by between 14 and 23 per cent between 2015 and 2030 (Wren et al., 2017), this needs to be accounted for in resource planning for the future. The Department of Health's capacity review and National Development Plan take into account projected population increases at the national level, but these need to be translated to the regional level to inform resource allocation plans for both the acute and non-acute sectors.

In addition to recent and projected population increases, Ireland is experiencing a shift in the age structure of the population; the largest relative increases are projected for the oldest age groups. These groups tend to be more intensive users of health and social care services, and this will have implications for resource allocation decisions, especially for the home care and LTRC sectors. Between 2015 and 2030, the population aged 65 years and over is projected to increase by between 58 and 63 per cent, with over a doubling of the population aged 85 years 
and older projected (Wren et al., 2017). Planning needs to place greater emphasis on social care and long-term care.

The projected population increase will also potentially result in some opportunities in terms of workforce planning. The working aged population, in absolute terms, is projected to increase by between 308,000 and 568,000 between 2015 and 2030 (Wren et al., 2017), proving policymakers with a potential workforce required to provide the increased health and social care services needed.

\subsection{CONCLUSIONS}

Overall, the findings of this project have added significantly to the evidence base on health and social care in Ireland. We find that there is substantial variation in the supply of non-acute services across regions, which does not reflect relative need. The east of the country fares particularly badly. Having been selected for a more detailed examination of the cases of home care and LRTC, we have found an association between greater supply of such services outside hospitals and reduced duration of stay in acute hospitals, which is stronger for some patient groups than others. These headline findings from the two reports of this project suggest that hospital performance in Ireland may be driven by factors outside the control of the hospitals, as well as within-hospital factors. Consequently, the system of $A B F$, which is being rolled out across acute hospitals, may penalise hospitals inappropriately and further add to challenges for areas, which already have relatively inadequate non-acute services.

Inequitable supply of non-acute care across Ireland has arisen in an apparently arbitrary, historical manner, and reflects the absence of any system of planning for population health need. Ireland is unusual in not having a transparent and consistent system of resource allocation related to analysis of need. With rapidly increasing demographic pressures on the healthcare system and planned large capacity increases, introducing such a resource allocation method is a priority to optimise care and achieve cost-effective healthcare expenditure. Factors which are required to facilitate this transition to better resource allocation include: improved data collection; an evidence-based approach to potential substitution between differing services such as home care and hospital care; systematic methods to integrate care across settings; planning for the development and expansion of the healthcare workforce; and regular updating of resource allocation in line with regional population projections. 



\section{REFERENCES}

Allison, P. D. and R. P. Waterman (2002). 'Fixed-effects negative binomial regression models', Sociological Methodology, Vol. 32, pp. 247-265.

Ambugo, E. A. and T. P. Hagen (2019). 'Effects of introducing a fee for inpatient overstays on the rate of death and readmissions across municipalities in Norway', Social Science and Medicine, Vol. 230, pp. 309-317.

Asaria, M., R. Cookson, R. Fleetcroft and S. Ali (2016). 'Unequal socioeconomic distribution of the primary care workforce: Whole-population small area longitudinal study', BMJ Open, Vol. 6, No. 1, e008783.

Bagust, A., M. Place and J. W. Posnett (1999). 'Dynamics of bed use in accommodating emergency admissions: Stochastic simulation model', BMJ, Vol. 319, No. 7203, pp. 155-8.

Balentine, C. J., A. D. Naik, C. N. Robinson, N. J. Peterson, G. J. Chen, D. H. Berger and D. A. Anaya (2014). 'Association of high-volume hospitals with greater likelihood of discharge to home following colorectal surgery', JAMA Surgery, Vol. 149, No. 3, pp. 244-251.

Barr, B., C. Bambra and M. Whitehead (2014). 'The impact of NHS resource allocation policy on health inequalities in England 2001-11: Longitudinal ecological study', BMJ, Vol. 348, g3231.

Besstremyannaya, G. (2016). 'Differential effects of declining rates in a per diem payment system', Health Econ, Vol. 25, No. 12, pp. 1599-1618.

Bhandari, A. and T. Wagner (2006). 'Self-reported utilization of health care services: Improving measurement and accuracy', Med Care Res Rev, Vol. 63, No. 2, pp. 217235.

Blau, F. D. and L. M. Kahn (2017). 'The gender wage gap: Extent, trends, and explanations', Journal of Economic Literature, Vol. 55, No. 3, pp. 789-865.

Bloom, N., C. Propper, S. Seiler and J. Van Reenen (2015). 'The impact of competition on management quality: Evidence from public hospitals', The Review of Economic Studies, Vol. 82, No. 2, pp. 457-489.

Blundell, R., R. Griffith and F. Windmeijer (2002). 'Individual effects and dynamics in count data models', Journal of Econometrics, Vol. 108, pp. 113-131.

BMA (2015). Focus on the global sum allocation formula (Carr-Hill Formula), BMA.

Boden, D. G., A. Agarwal, T. Hussain, S. J. Martin, N. Radford, M. S. Riyat, K. So, Y. Su, A. Turvey and C. I. Whale (2015). 'Lowering levels of bed occupancy is associated with decreased inhospital mortality and improved performance on the 4-hour target in a UK District General Hospital', Emergency Medicine Journal, Vol. 33, No. 2, pp. 8590.

Borah, B. J. and A. Basu (2013). 'Highlighting differences between conditional and unconditional quantile regression approaches through an application to assess medication adherence', Health Econ, Vol. 22, No. 9, pp. 1052-1070. 
Borghans, I., R. Heijink, T. Kool, R. J. Lagoe and G. P. Westert (2008). 'Benchmarking and reducing length of stay in Dutch hospitals', BMC Health Serv Res, Vol. 8, pp. 220.

Brick, A., R. Layte, A. Nolan and M. J. Turner (2016). 'Differences in nulliparous caesarean section rates across models of care: A decomposition analysis', BMC Health Serv Res, Vol. 16, No. 1, pp. 239.

Brick, A., A. Nolan, J., O'Reilly and S. Smith (2010). Resource allocation, financing and sustainability in health care: Evidence for the Expert Group on Resource Allocation and Financing in the Health Sector, Dublin.

Brody, A. A., E. Ciemins, J. Newman and C. Harrington (2010). 'The effects of an inpatient palliative care team on discharge disposition', J Palliat Med, Vol. 13, No. 5, pp. 541548.

Burke, S., S. Thomas, S. Barry and C. Keegan (2014). 'Indicators of health system coverage and activity in Ireland during the economic crisis 2008-2014 - From 'more with less' to 'less with less", Health Policy, Vol. 117, No. 3, pp. 275-278.

Cahill, S., C. O'Nolan, D. O'Caheny and A. Bobersky (2015). An Irish national survey of dementia in long-term residential care.

Cameron, A. C. and P. K. Trivedi (2013). Regression analysis of count data, Cambridge University Press.

Cameron, C. A. and D. L. Miller (2015). 'A practitioner's guide to cluster-robust inference', Journal of Human Resources, Vol. 50, No. 2, pp. 317-372.

Canadian Institute for Health Information (2010). Health care in Canada 2010: Evidence of progress, but care not always appropriate, Ottowa.

Care Alliance Ireland (2018). 'Public provision of home care in Ireland - Update', Briefing Paper 2, Dublin: Care Alliance Ireland.

Carey, K. (2015). 'Measuring the hospital length of stay/readmission cost trade-off under a bundled payment mechanism', Health Econ, Vol. 24, No. 7, pp. 790-802.

Chitnis, X. A., T. Georghiou, S. Steventon and M. J. Bardsley (2013). 'Effect of a home-based end-of-life nursing service on hospital use at the end of life and place of death: A study using administrative data and matched controls', BMJ Supportive Palliative Care, Vol. 3, No. 4, pp. 422-430.

Christiansen, T. and K. Vrangbæk (2017). 'Hospital centralization and performance in Denmark - Ten years on', COHERE discussion paper No.7/2017.

Collins, B. (2015). Foundation trust and NHS trust mergers: 2010 to 2015, The King's Fund.

Comptroller and Auditor General (2018). Report on the accounts of the public services 2017.

Confalonieri, M., R. Trevisan, M. Demsar, L. Lattuada, C. Longo, R. Cifaldi, M. Jevnikar, M. Santagiuliana, L. Pelusi and R. Pistelli (2015). 'Opening of a respiratory intermediate care unit in a general hospital: Impact on mortality and other outcomes', Respiration, Vol. 90, No. 3, pp. 235-42.

Connolly, S., A. Nolan, B. Walsh and M.-A. Wren (2018). 'Universal GP care in Ireland: Potential cost implications', The Economic and Social Review, Vol. 49, No. 1, pp. 93109. 
Connolly, S. and O'Shea, E. (2015). 'The impact of dementia on length of stay in acute hospitals in Ireland', Dementia, Vol. 14, No. 5, pp. 650-658.

Costa-Font, J., Elvira, D. and Mascarilla-Miró, O. (2009). “'Ageing in place"? Exploring elderly people's housing preferences in Spain', Urban Studies, Vol. 46, No. 2, pp. 295-316.

Costa-Font, J., S. Jimenez-Martin and C. Vilaplana (2018). 'Does long-term care subsidization reduce hospital admissions and utilization?', J Health Econ, Vol. 58, pp. 43-66.

Cowell, F. A. and E. Flachaire (2007). 'Income distribution and inequality measurement: The problem of extreme values', Journal of Econometrics, Vol. 141, No. 2, pp. 10441072.

Crawford, R., G. Stoye and B. Zaranko (2018). The impact of cuts to social care spending on the use of accident.

CSO (2019). System of health accounts: Ireland's system of health accounts, annual results 2017, 2019, available at:

https://www.cso.ie/en/releasesandpublications/er/sha/systemofhealthaccounts2 $017 /$.

Cullinan, J., K. Denny and D. Flannery (2018). 'A distributional analysis of upper secondary school performance', UCD Geary Instiute Discussion Paper, WP2018/08.

Dahl, U., A. Steinsbekk and R. Johnsen (2015). 'Effectiveness of an intermediate care hospital on readmissions, mortality, activities of daily living and use of health care services among hospitalized adults aged 60 years and older - A controlled observational study', BMC Health Serv Res, Vol. 15, pp. 351.

Dedhia, P., S. Kravet, J. Bulger, T. Hinson, A. Sridharan, K. Kolodner, S. Wright and E. Howell (2009). 'A quality improvement intervention to facilitate the transition of older adults from three hospitals back to their homes', J Am Geriatr Soc, Vol. 57, No. 9, pp. 1540-1546.

Department of Health (2017a). Health in Ireland: Key trends 2017, Dublin.

Department of Health (2017b). HSE non-capital vote allocation in millions of euro, 2009 to 2015.

Department of Health (2017c). Trends in public / private patient activity in public acute hospitals.

Department of Health (2017d). Working together for health: A national strategic framework for health workforce planning.

Department of Health (2018a). Health service capacity review 2018: Review of health demand and capacity requirements in Ireland to 2031.

Department of Health (2018b). Independent expert review of delayed discharges.

Department of Health (2019). Sláintecare Action Plan 2019.

Department of Health and Children (2002). Acute hospital bed capacity: A national review, Dublin. 
Downing, A., M. Lansdown, R. M. West, J. D. Thomas, G. Lawrence and D. Forman (2009). 'Changes in and predictors of length of stay in hospital after surgery for breast cancer between 1997/98 and 2004/05 in two regions of England: A populationbased study', BMC Health Serv Res, Vol. 9, pp. 202.

Droog, E., C. Foley, O. Healy, C. Buckley, M. Boyce, S. McHugh and J. P. Browne (2018). 'Perspectives on the underlying drivers of urgent and emergency care reconfiguration in Ireland', Int J Health Plann Manage, Vol. 33, No. 2, pp. 364-379.

Échevin, D. and B. Fortin (2014). 'Physician payment mechanisms, hospital length of stay and risk of readmission: Evidence from a natural experiment', $J$ Health Econ, Vol. 36, pp. 112-124.

Edwards, N. (2017). 'What's behind delayed transfers of care?', Briefing, Nuffield Trust.

Eighan, J., B. Walsh, S. Smith, M. A. Wren, S. Barron and E. Morgenroth (2019). 'A profile of physiotherapy supply in Ireland', Ir J Med Sci, Vol. 188, No. 1, pp. 19-27.

Eriksson, C. O., R. C. Stoner, K. B. Eden, C. D. Newgard and J. M. Guise (2017). 'The association between hospital capacity strain and inpatient outcomes in highly developed countries: A systematic review', J Gen Intern Med, Vol. 32, No. 6, pp. 686-696.

Farrar, S., D. Yi, M. Sutton, M. Chalkley, J. Sussex and A. Scott (2009). 'Has payment by results affected the way that English hospitals provide care? Difference-indifferences analysis', BMJ, Vol. 339, b3047.

Fernandez, J.-L., A. McGuire and M. Raikou (2018). 'Hospital coordination and integration with social care in England: The effect on post-operative length of stay', Journal of health economics, Vol. 61, pp. 233-243.

Fernandez, J. L. and J. Forder (2008). 'Consequences of local variations in social care on the performance of the acute health care sector', Applied Economics, Vol. 40, No. 12.

Fernandez, J. L. and J. Forder (2015). 'Local variability in long-term care services: Local autonomy, exogenous influences and policy spillovers', Health Econ, Vol. 1, pp. 146-57.

Fernandez, J. L., T. Snell and G. Wistow (2013). Changes in the patterns of social care provision in England: 2005/6 to 2012/13, Personal Social Services Research Unit, London.

Fisher, S. R., Y.-f. Kuo, J. E. Graham, K. J. Ottenbacher and G. V. Ostir (2010). 'Early ambulation and length of stay in older adults hospitalized for acute illness', Archives of Internal Medicine, Vol. 170, No. 21, pp. 1942-1943.

Forder, J. (2009). 'Long-term care and hospital utilisation by older people: An analysis of substitution rates', Health Econ, Vol. 18, No. 11, pp. 1322-1338.

Forder, J., J. Caiels, J. Harlock, G. Wistow, G. Malisauskaite, M. Peters, J. Marczak, F. D’Amico, J.-L. Fernández, R. Fitzpatrick and J. Karen (2018). A system-level evaluation of the Better Care Fund: Final report.

Forder, J., K. Gousia and E.-C. Saloniki (2019). 'The impact of long-term care on primary care doctor consultations for people over 75 years', The European Journal of Health Economics, Vol. 20, No. 3, pp. 375-387. 
Fried, T. R., C. van Doorn, J. R. O'Leary, M. E. Tinetti and M. A. Drickamer (2000). 'Older persons' preferences for home vs hospital care in the treatment of acute illness', Archives of Internal Medicine, Vol. 160, No. 10, pp. 1501-1506.

Gannon, B. (2005). 'Testing for variation in technical efficiency of hospitals in Ireland', The Economic and Social Review, Vol. 36, No. 3, pp. 273-294.

Gaughan, J., H. Gravelle, R. Santos and L. Siciliani (2017a). 'Long-term care provision, hospital bed blocking, and discharge destination for hip fracture and stroke patients', Int J Health Econ Manag, Vol. 28, No. 10, pp. 017-9214.

Gaughan, J., H. Gravelle and L. Siciliani (2015). 'Testing the bed-blocking hypothesis: does nursing and care home supply reduce delayed hospital discharges?' Health economics, Vol. 24, Suppl 1, pp. 32-44.

Gaughan, J., H. Gravelle and L. Siciliani (2017b). 'Delayed discharges and hospital type: Evidence from the English NHS', Fiscal Studies, Vol. 38, No. 3, pp. 495-519.

Glance, L. G., P. W. Stone, D. B. Mukamel and A. W. Dick (2011). 'Increases in mortality, length of stay, and cost associated with hospital-acquired infections in trauma patients', Archives of Surgery (Chicago, III. : 1960), Vol. 146, No. 7, pp. 794-801.

Gonçalves, J. and F. Weaver, F. (2017). 'Effects of formal home care on hospitalizations and doctor visits', International Journal of Health Economics and Management, Vol. 17, No. 2, pp. 203-233.

Government of Ireland (2018a). Project Ireland 2040: National development plan 20182027, Dublin.

Government of Ireland (2018b). Sláintecare implementation strategy, Dublin: Government of Ireland.

Gozalo, P., N. E. Leland, T. J. Christian, V. Mor and J. M. Teno (2015). 'Volume matters: Returning home after hip fracture', J Am Geriatr Soc, Vol. 63, No. 10, pp. 20432051.

Green, M. A., D. Dorling, J. Minton and K. E. Pickett (2017). 'Could the rise in mortality rates since 2015 be explained by changes in the number of delayed discharges of NHS patients?' Journal of Epidemiology and Community Health, Vol. 71, No. 11, pp. 1068-1071.

Ham, C., N. York, S. Sutch and R. Shaw (2003). 'Hospital bed utilisation in the NHS, Kaiser Permanente, and the US Medicare programme: Analysis of routine data', BMJ, Vol. 327, No. 7426, pp. 1257.

Hanly, P. and C. Sheerin (2017). 'Valuing informal care in Ireland: Beyond the traditional production boundary', Economic and Social Review, Vol. 48, No. 3 pp. 337-464.

Harmon, C. and B. Nolan (2001). 'Health insurance and health services utilization in Ireland', Health Econ, Vol. 10, No. 2, pp. 135-145.

Harold, J., J. Cullinan and S. Lyons (2017). 'The income elasticity of household energy demand: A quantile regression analysis', Applied Economics, Vol. 49, No. 54, pp. 5570-5578. 
Healthcare Pricing Office (2016a). Activity in acute public hospitals in Ireland annual report, 2015, Dublin: Healthcare Pricing Office.

Healthcare Pricing Office (2016b). Coding notes: National audit of admitted patient information, Dublin: Healthcare Pricing Office.

Heckley, G., U.-G. Gerdtham and G. Kjellsson (2016). 'A general method for decomposing the causes of socioeconomic inequality in health', J Health Econ, Vol. 48, pp. 89106.

Hilbe, J. M. (2011). Negative binomial regression, Cambridge University Press.

Health In-Patient Enquiry (HIPE) (2009). Activity in acute public hospitals in Ireland: 2008 annual report, Dublin: The Economic an Social Research Institute.

Health Information and Quality Authority (HIQA) (2009). Report of the investigation into the quality and safety of services and supporting arrangements provided by the Health Service Executive at the Mid-Western Regional Hospital Ennis.

Health Information and Quality Authority (HIQA) (2011). Report of the investigation into the quality and safety of services and supporting arrangements provided by the Health Service Executive at Mallow General Hospital.

Houses of the Oireachtas Committee on the Future of Healthcare (2017). Sláintecare report, Dublin.

HSE (2012). HSE implementation of the HIQA Ennis and Mallow reports.

HSE (2019). Injury units, 2019, available at: https://www.hse.ie/eng/about/who/acutehospitals-division/patient-care/injury-units/.

Huckfeldt, P. J., N. Sood, J. J. Escarce, D. C. Grabowski and J. P. Newhouse (2014). 'Effects of Medicare payment reform: Evidence from the home health interim and prospective payment systems', J Health Econ, Vol. 34, pp. 1-18.

Ilinca, S., K. Leichsenring and R. Rodrigues (2015). From care in homes to care at home: European experiences with (de)institutionalisation in long-term care, Vienna.

Irish Medical Organisation (2019). Statement: IMO reaches $€ 210 \mathrm{~m}$ deal with govt for GP services, 2019, available at: https://www.imo.ie/news-media/news-pressreleases/2019/statement-imo-reaches-210/index.xml.

Jayne, D. G., H. C. Thorpe, , J. Copeland, P. Quirke, J. M. Brown and P. J. Guillou (2010). 'Five-year follow-up of the Medical Research Council CLASICC trial of laparoscopically assisted versus open surgery for colorectal cancer', Br J Surg, Vol. 97, No. 11, pp. 1638-1645.

Kapur, K. (2019). 'Private health insurance in Ireland: Trends and determinants', UCD Centre For Economic Research, Working Paper Series, WP19/03.

Keegan, C., A. Brick, B. Walsh, A. Bergin, J. Eighan and M.-A. Wren (2018a). 'How many beds? Capacity implications of hospital care demand projections in the Irish hospital system, 2015-2030', Int J Health Plann Manage, pp. 1-14.

Keegan, C., A. Brick and M.-A. Wren (2018b). An examination of activity in public and private hospitals in Ireland, 2015, ESRI Working Paper 601, Dublin: ESRI. 
Keegan, C. and S. Smith (2013). 'The length of stay of in-patient stroke discharges in Irish acute hospitals', Economic and Social Review, Vol. 44, No. 3, pp. 351-370.

Keegan, C., S. Thomas, C. Normand and C. Portela (2013). 'Measuring recession severity and its impact on healthcare expenditure', Int J Health Care Finance Econ, Vol. 13, No. 2, pp. 139-155.

Keogh, F., M. Pierce, K. Neylon and P. Fleming (2018). 'Intensive home care packages for people with dementia: A realist evaluation protocol', BMC Health Serv Res, Vol. 18, No. 1, 289.

Kiersey, R. A. and A. Coleman (2017). Approaches to the regulation and financing of home care services in four European countries: An evidence review, Dublin: Health Research Board.

Kjellsson, G., P. Clarke and U. G. Gerdtham (2014). 'Forgetting to remember or remembering to forget: a study of the recall period length in health care survey questions', J Health Econ, Vol. 35, pp. 34-46.

Kossovsky, M. P., F. P. Sarasin, P. Chopard, M. Louis-Simonet, P. Sigaud, T. V. Perneger and J. M. Gaspoz (2002). 'Relationship between hospital length of stay and quality of care in patients with congestive heart failure', Quality and safety in health care, Vol. 11, No. 3, pp. 219-223.

Kroneman, M. and J. J. Siegers (2004). 'The effect of hospital bed reduction on the use of beds: a comparative study of 10 European countries', Soc Sci Med, Vol. 59, No. 8, pp. 1731-1740.

Laudicella, M., B. Walsh, A. Munasinghe and O. Faiz (2016). 'Impact of laparoscopic versus open surgery on hospital costs for colon cancer: A population-based retrospective cohort study', BMJ Open, Vol. 6, No. 11.

Layte, R., M. Barry, K. Bennett, A. Brick, E. Morgenroth, C. Normand, J. O'Reilly, S. Thomas, L. Tilson, M. M. Wiley and M.-A. Wren (2009). Projecting the impact of demographic change on the demand for and delivery of health care in Ireland, ESRI Research Series 13, Dublin: ESRI.

Lehto, J., K. Vrangbaek and U. Winblad (2015). 'The reactions to macro-economic crises in Nordic health system policies: Denmark, Finland and Sweden, 1980-2013', Health Econ Policy Law, Vol. 10, No. 1, pp. 61-81.

Li, B., C. M. Couris, J.-M. Januel, K. Fushimi, P. Graham, P. Hider, , V. Sundararajan and H. Quan (2011). 'Updating and validating the Charlson comorbidity index and score for risk adjustment in hospital discharge abstracts using data from 6 countries', American Journal of Epidemiology, Vol. 173, No. 6, pp. 676-682.

Lichtenberg, F. R. (2012). 'Is home health care a substitute for hospital care?', Home Health Care Serv $Q$, Vol. 31, No. 1, pp. 84-109.

Longo, F., L. Siciliani, G. Moscelli and H. Gravelle (2019). 'Does hospital competition improve efficiency? The effect of the patient choice reform in England', Health Economics, Vol. 28, No. 5, pp. 618-640.

Ma, Y. and A. Nolan (2016). 'Public healthcare entitlements and healthcare utilisation among the older population in Ireland', Health Economics, Vol. 25, No. 11. 
Madsen, F., S. Ladelund and A. Linneberg (2014). 'High levels of bed occupancy associated with increased inpatient and thirty-day hospital mortality in Denmark', Health Aff, Vol. 33, No. 7, pp. 1236-1244.

Martin, S. and P. Smith (1996). 'Explaining variations in inpatient length of stay in the National Health Service', J Health Econ, Vol. 15, No. 3, pp. 279-304.

Martin, S., A. Street, L. Han and J. Hutton (2016). 'Have hospital readmissions increased in the face of reductions in length of stay? Evidence from England', Health Policy, Vol. 120, No. 1, pp. 89-99.

McCoy, D., S. Godden, A. M. Pollock and C. Bianchessi (2007). 'Carrot and sticks? The Community Care Act (2003) and the effect of financial incentives on delays in discharge from hospitals in England', J Public Health (Oxf), Vol. 29, No. 3, pp. 281287.

McHugh, S., E. Droog, C. Foley, M. Boyce, O. Healy and J. P. Browne (2019). 'Understanding the impetus for major systems change: A multiple case study of decisions and nondecisions to reconfigure emergency and urgent care services', Health Policy, Vol. 123, No. 8, pp. 728-736.

McLoughlin, P. (2014). Review of measures to reduce costs in the private health insurance market 2014: Independent report to the Minister for Health and Health Insurance Council, Dublin.

Medical Council (2019). Medical workforce intelligence report: A report on the 2016 and 2017 annual registration retention and voluntary registration withdrawal surveys, Dublin: Medical Council.

Mladovsky, P., D. Srivastava, J. Cylus, M. Karanikolos, T. Evetovits, S. Thomson and M. McKee (2012). Health policy responses to the financial crisis in Europe, Copenhagen: World Health Organization Europe.

Mohan, G., A. Nolan and S. Lyons (2019). 'An investigation of the effect of accessibility to general practitioner services on healthcare utilisation among older people', Social Science and Medicine, Vol. 220, pp. 254-263.

Murphy, C. M., B. J. Whelan and C. Normand (2015). 'Formal home-care utilisation by older adults in Ireland: Evidence from the Irish Longitudinal Study on Ageing (TILDA)', Health and Social Care in the Community, Vol. 23, No. 4, pp. 408-418.

National Audit Office (2016). Discharging older patients from hospital, National Audit Office.

Neiterman, E., W. P. Wodchis and I. L. Bourgeault (2015). 'Experiences of older adults in transition from hospital to community', Can J Aging, Vol. 34, No. 1, pp. 90-99.

Nolan, A. (2011). 'An extension in eligibility for free primary care and avoidable hospitalisations: a natural experiment', Soc Sci Med, Vol. 73, No. 7, pp. 978-985.

Nolan, A., S. Barry, S. Burke and S. Thomas (2014). The impact of the financial crisis on the health system and health in Ireland, Copenhagen, Denmark: World Health Organization Europe. 
Norton, E. C., C. H. Van Houtven, R. C. Lindrooth, S. L. Normand and B. Dickey (2002). 'Does prospective payment reduce inpatient length of stay?', Health Econ, Vol. 11, No. 5, pp. 377-387.

O'Donnell, O., S. O'Neill, T. Van Ourti and B. Walsh (2016). 'Conindex: Estimation of concentration indices', Stata J, Vol. 16, No. 1, pp. 112-138.

O'Reilly, J. and M. Wiley (2010). 'Who's that sleeping in my bed? Potential and actual utilization of public and private in-patient beds in Irish acute public hospitals', Journal of Health Services Research and Policy, Vol. 15, No. 4, pp. 210-214.

O'Callaghan, M. E., L. Zgaga, D. O'Ciardha and T. O'Dowd (2018). 'Free children's visits and general practice attendance', The Annals of Family Medicine, Vol. 16, No. 3, pp. 246-249.

O’Regan, E. (2014). 'Opposition grows to plan for universal healthcare', Irish Independent, 7 April 2014.

Öckert, B., H. Oosterbeek and P. Fredriksson (2012). 'Long-term effects of class size', The Quarterly Journal of Economics, Vol. 128, No. 1, pp. 249-285.

OECD (2012). OECD health data 2012. Definitions, sources and methods: Curative (acute) care beds in hospitals.

OECD (2017a). Hospital beds (indicator), available at:

https://data.oecd.org/healtheqt/hospital-beds.htm.

OECD (2017b). Length of hospital stay (indicator), available at:

https://data.oecd.org/healthcare/length-of-hospital-stay.htm.

OECD (2018). Health at a glance: Europe 2018.

Olsen, M. A., F. Tian, A. E. Wallace, K. B. Nickel, D. K. Warren, V. J. Fraser, N. Selvamand and B. H. Hamilton (2017). 'Use of quantile regression to determine the impact on total health care costs of surgical site infections following common ambulatory procedures', Annals of Surgery, Vol. 265, No. 2, pp. 331-339.

PA Consulting (2007). Acute hospital bed capacity review: A preferred health system in Ireland to 2020, Dublin.

PA Consulting (2018). Health service capacity review 2018, Dublin: Department of Health.

Private Hospitals Association (2016). PHA submission to the Oireachtas Commitee on the Future of Health Care, 2016, available at: http://privatehospitals.ie/wpcontent/uploads/2017/01/Private-Hospitals-Association-Submission-August2016.pdf.

Private Hospitals Association (2017). Private hospitals' contribution to the Irish healthcare system, 2017, available at: http://privatehospitals.ie/private-hospitals-in-ireland/.

Propper, C. and J. Van Reenen (2010). 'Can pay regulation kill? Panel data evidence on the effect of labor markets on hospital performance', Journal of Political Economy, Vol. 118 , No. 2, pp. 222-273.

Public Service Pay Commission (2018). Peport of the Public Service Pay Commission recruitment and retention Module 1. 
Rae, B., W. Busby and P. H. Millard (2007). 'Fast-tracking acute hospital care - From bed crisis to bed crisis', Aust Health Rev, Vol. 31, No. 1, pp. 50-62.

Redmond, P. and S. McGuinness (2018). 'The gender wage gap in europe: Job preferences, gender convergence and distributional effects', Oxford Bulletin of Economics and Statistics, Vol. 81, No. 3, pp. 564-587.

Rokicki, S., J. Cohen, G. Fink, J.A. Salomon and M. B. Landrum (2018). 'Inference with difference-in-differences with a small number of groups: A review, simulation study, and empirical application using SHARE data', Med Care, Vol. 56, No. 1, pp. 97-105.

Schilling, P. L., D. A. Campbell, Jr., M. J. Englesbe and M. M. Davis (2010). 'A comparison of in-hospital mortality risk conferred by high hospital occupancy, differences in nurse staffing levels, weekend admission, and seasonal influenza', Med Care, Vol. 48, No. 3, pp. 224-32.

Sexton, E. and D. Bedford (2016). 'GP supply, deprivation and emergency admission to hospital for COPD and diabetes complications in counties across Ireland: An exploratory analysis', Irish Journal of Medical Science, Vol. 185, No. 2, pp. 453-461.

Siciliani, L., V. Moran and M. Borowitz (2014). 'Measuring and comparing health care waiting times in OECD countries', Health Policy, Vol. 118, No. 3, pp. 292-303.

Siciliani, L., P. Sivey and A. Street (2013). 'Differences in length of stay for hip replacement between public hospitals, specialised treatment centres and private providers: Selection or efficiency?', Health economics, Vol. 22, No. 2, pp. 234-242.

Siegler, J. E., A. K. Boehme, B. D. Fowler, A. J. George, D. J. Monlezun, K. C. Albright, T. M. Beasley and S. Martin-Schild (2013). 'Inpatient rehabilitation centers and concern for increasing volume of ischemic stroke patients requiring rehabilitation', South Med J, Vol. 106, No. 12, pp. 693-696.

Smith, S., S. Barron, M.-A. Wren, B. Walsh, , E. Morgenroth, J. Eighan and S. Lyons (2019). Geographic profile of healthcare needs and non-acute healthcare supply in Ireland, ESRI Research Series No. 90, Dublin: ESRI.

Sutherland, J. M. and R. T. Crump (2013). 'Alternative level of care: Canada's hospital beds, the evidence and options', Healthcare policy = Politiques de sante, Vol. 9, No. 1, pp. 26-34.

Tefft, N. (2011). 'Insights on unemployment, unemployment insurance and mental health', J Health Econ, Vol. 30, No. 2, pp. 258-264.

Teljeur, C., S. Thomas, F. D. O'Kelly and T. O'Dowd (2010). 'General practitioner workforce planning: Assessment of four policy directions', BMC Health Serv Res, Vol. 10, No. $1,148$.

Teljeur, C., E. Tyrrell, A. Kelly, T. O'Dowd and S. Thomas (2014). 'Getting a handle on the general practice workforce in Ireland', Ir J Med Sci, Vol. 183, No. 2, pp. 207-213.

The King's Fund (2015). The NHS in a nutshell: The number of hospital beds.

The King's Fund (2017). The number of hospital beds, 2017, available at: https://www.kingsfund.org.uk/projects/nhs-in-a-nutshell/hospital-beds. 
Thomeer, M. B., S. Mudrazija and J. L. Angel (2016). 'Relationship status and long-term care facility use in later life', The Journals of Gerontology. Series B, Psychological Sciences and Social Sciences, Vol. 71, No. 4, pp. 711-723.

Timonen Doyle, V. M. (2008). 'From the workhouse to the home: Evolution of care policy for older people in Ireland', International Journal of Sociology and Social Policy, Vol. 28 , No. 3/4, pp. 76-89.

Timonen, V. and M. Doyle (2007). 'Worlds apart? Public, private and non-profit sector providers of domiciliary care for older persons in Ireland', Journal of Aging Studies, Vol. 21, No. 3, pp. 255-265.

Timonen, V., M. Doyle and C. O'Dwyer (2012). 'Expanded, but not regulated: Ambiguity in home-care policy in Ireland', Health and Social Care in the Community, Vol. 20, No. 3, pp. 310-318.

Tussing, D. A. and M.-A. Wren (2006). How Ireland cares: The case for health care reform, Dublin: New Island.

Van Doorslaer, E. and X. Koolman (2004). 'Explaining the differences in income-related health inequalities across European countries', Health Econ, Vol. 13, No. 7, pp. 609628.

Van Houtven, C. H. and E. C. Norton (2004). 'Informal care and health care use of older adults', J Health Econ, Vol. 23, No. 6, pp. 1159-1180.

Vega, A., S. O'Shea, C. Murrin and A. Staines (2010). Towards the development of a resource allocation model for primary, continuing and community care in the health services, Dublin: Dublin City University.

VHI (2017). Annual report and accounts 2016.

Walsh, B., A. Nolan, A. Brick and C. Keegan (2019). 'Did the expansion of free GP care impact demand for emergency department attendances? A difference-in-differences analysis', Social Science and Medicine, Vol. 222, pp. 101-111.

Wanless, D. (2002). Securing our future health: Taking a long-term view, London: HM Treasury.

Wren, M.-A., P. Gillespie, S. Smith, K. Kearns, S. Crichton, D. Parkin and A. Hickey (2014). Towards earlier discharge, better outcomes, lower cost: Stroke rehabilitation in Ireland, Dublin: ESRI.

Wren, M.-A., C. Keegan, B. Walsh, A. Bergin, J. Eighan, A. Brick, S. Connolly, D. Watson and J. Banks (2017). Projections of demand for healthcare in Ireland, 2015-2030: First report from the Hippocrates Model, Dublin: ESRI Research Series No. 67. 



\section{APPENDIX 1}

\section{Additional data}

TABLE A.1 RESIDENCE IDS IN HIPE

\begin{tabular}{|c|c|}
\hline No fixed abode* & Clare \\
\hline International* & Carlow \\
\hline Cork City & Cavan \\
\hline Cork County & Galway City \\
\hline Donegal & Galway County \\
\hline Dublin $1^{ \pm}$ & Kerry \\
\hline Dublin $3^{ \pm}$ & Kildare \\
\hline Dublin $5^{ \pm}$ & Kilkenny \\
\hline Dublin $7^{ \pm}$ & Laois \\
\hline Dublin $9^{ \pm}$ & Leitrim \\
\hline Dublin $11^{ \pm}$ & Limerick City \\
\hline Dublin $13^{ \pm}$ & Limerick County \\
\hline Dublin $15^{ \pm}$ & Longford \\
\hline Dublin $17^{ \pm}$ & Louth \\
\hline Dublin $2^{\neq}$ & Mayo \\
\hline Dublin $4^{\neq}$ & Meath \\
\hline Dublin $6^{\neq}$ & Monaghan \\
\hline Dublin $8^{\neq}$ & Offaly \\
\hline Dublin $10^{\neq}$ & Roscommon \\
\hline Dublin $12^{\neq}$ & Sligo \\
\hline Dublin $14^{\neq}$ & Tipperary North Riding \\
\hline Dublin $16^{\neq}$ & Tipperary South Riding \\
\hline Dublin $18^{\neq}$ & Waterford City \\
\hline Dublin $20^{\neq}$ & Waterford County \\
\hline Dublin $22^{\neq}$ & Westmeath \\
\hline Dublin $24^{\neq}$ & Wexford \\
\hline Dun Laoghaire ${ }^{\neq}$ & Wicklow \\
\hline
\end{tabular}

Notes: * Not included in analyses; ${ }^{ \pm}$Dublin North; ${ }^{\ddagger}$ Dublin South. 


\begin{tabular}{|c|c|c|c|}
\hline Hospital & Years available & Comment & Tier 1 ED in 2015 \\
\hline Bantry General Hospital & 2009-2010, 2012-2014 & 24 Hours ED closed in July 2013 & No \\
\hline Beaumont Hospital & 2005-2015 & & Yes \\
\hline Blackrock Hospice & 2010-2014 & Hospice & No \\
\hline Cavan General Hospital & 2005-2015 & & Yes \\
\hline Cherry Orchard Hospital, Ballyfermot & 2005-2012 & Rehabilitation hospital & No \\
\hline Connolly Hospital, Blanchardstown & 2005-2015 & & Yes \\
\hline Coombe Women and Infants University Hospital & 2005-2015 & Maternity hospital & Yes. Maternity ED \\
\hline Cork University Hospital & 2005-2015 & Includes Cork University Maternity Hospital & Yes \\
\hline Ennis General Hospital & 2005-2014 & 24 Hours ED closed April 2009. Services transferred to University Hospital Limerick & No \\
\hline Erinville Hospital, Cork & 2005-2007 & Maternity services transferred to Cork University Maternity Hospital March 2007 & No \\
\hline Hume Street Skin Cancer Hospital & 2006 & Closed November 2006. Facilities were moved to St. Vincent's University Hospital & No \\
\hline Incorporated Orthopaedic Hospital, Clontarf & 2005-2015 & Rehabilitation hospital & No \\
\hline Kerry General Hospital, Tralee & 2005-2015 & & Yes \\
\hline Letterkenny General Hospital & 2005-2015 & & Yes \\
\hline Lourdes Orthopaedic Hospital, Kilcreene & 2005-2015 & Rehabilitation hospital & No \\
\hline Louth County Hospital, Dundalk & 2005-2015 & 24 Hours ED closed June 2010 & No \\
\hline Mallow General Hospital & 2005-2015 & 2014: Returned $96.7 \%$ of discharges & No \\
\hline Mater Misericordiae University Hospital & 2005-2015 & & Yes \\
\hline Mayo General Hospital & 2005-2015 & & Yes \\
\hline Mercy University Hospital - Cork & 2005-2015 & & Yes \\
\hline Merlin Park Hospital, Galway & 2005-2010 & Merged into University Hospital Galway in 2010 & No \\
\hline Midland Regional Hospital - Mullingar & 2005-2015 & & Yes \\
\hline Midland Regional Hospital - Portlaoise & 2005-2015 & & Yes \\
\hline Midland Regional Hospital - Tullamore & 2005-2015 & & Yes \\
\hline Midwestern Regional Hospital, Nenagh & 2005-2015 & 24 Hours ED closed April 2009. Services transferred to University Hospital Limerick & No \\
\hline Midwestern Regional Maternity Hospital & 2005-2015 & Maternity hospital & No \\
\hline Midwestern Regional Orthopaedic Hospital & 2005-2014 & Rehabilitation hospital & No \\
\hline Monaghan General Hospital & 2005-2015 & 24 Hours ED closed July 2009 & No \\
\hline Naas General Hospital & 2005-2015 & & Yes \\
\hline The National Maternity Hospital at Holles St & 2005-2015 & Maternity hospital & Yes. Maternity ED \\
\hline National Orthopaedic Hospital, Cappagh & 2005-2015 & Rehabilitation hospital & No \\
\hline National Rehabilitation Hospital (NRH) & 2005-2015 & Rehabilitation hospital & No \\
\hline Our Lady of Lourdes Hospital - Drogheda & 2005-2015 & 2012: Returned $97.1 \%$ of discharges & Yes \\
\hline Our Lady's Children's Hospital - Crumlin & 2005-2015 & Paediatric hospital & Yes \\
\hline Our Lady's Hospice, Harold's Cross & 2005-2014 & & No \\
\hline Our Lady's Hospital, Cashel & 2005-2006 & Services transferred to South Tipperary General Hospital January 2007 & No \\
\hline Our Lady's Hospital - Navan & 2005-2015 & 24 Hours ED closed for a time in 2013 & Yes \\
\hline
\end{tabular}




\begin{tabular}{|c|c|c|c|}
\hline Hospital & Years available & Comment & Tier 1 ED in 2015 \\
\hline Peamount Hospital, Newcastle & 2005-2015 & Rehabilitation hospital & No \\
\hline Portiuncula Hospital - Ballinasloe & 2005-2015 & & Yes \\
\hline Roscommon County Hospital & 2005-2015 & 24 Hours ED closed July 2011 & No \\
\hline The Rotunda Hospital Dublin & 2005-2015 & Maternity hospital & Yes. Maternity ED \\
\hline Royal Victoria Eye and Ear Hospital & 2005-2015 & $\begin{array}{l}\text { ED for ophthalmology open 08:00-20:00 Monday Friday; ED for ear, nose, throat, } \\
\text { head and neck open 09:00-16:00 Monday-Friday }\end{array}$ & No \\
\hline Sligo General Hospital & 2005-2015 & & Yes \\
\hline South Infirmary Victoria Hospital & 2005-2015 & 24 Hours ED closed July 2012 & No \\
\hline South Tipperary General Hospital & 2005-2015 & & Yes \\
\hline St. Columcilles Hospital, Loughlinstown & 2005-2015 & 24 Hours ED closed 27th Nov 2013 & No. \\
\hline St. Finbarrs Hospital & 2005-2015 & Maternity services transferred to Cork University Maternity Hospital March 2007 & No \\
\hline St. James' Hospital & 2005-2015 & & Yes \\
\hline St. Johns Hospital, Limerick & 2005-2015 & $\begin{array}{l}24 \text { Hours ED closed September 2013. Services transferred to University Hospital } \\
\text { Limerick }\end{array}$ & No \\
\hline St. Joseph's Hospital, Raheny & 2009-2015 & Started reporting to HIPE 1 January 2009 & No \\
\hline St. Josephs Unit, Harold's Cross & 2005-2015 & & No \\
\hline St. Luke's and St. Annes Hospital & 2005-2015 & & No \\
\hline St. Luke's General Hospital - Kilkenny & 2005-2015 & & Yes \\
\hline St. Mary's Orthopaedic Hospital, Gurranebraher & 2005-2011 & Rehabilitation hospital & No \\
\hline St. Michaels Hospital, Dun Laoghaire & 2005-2015 & ED open 08.00-20.00 7 days a week & No \\
\hline St. Vincent's University Hospital & 2005-2015 & & Yes \\
\hline Tallaght University Hospital - Adult ED & 2005-2015 & & Yes \\
\hline Tallaght University Hospital - Paediatric ED & 2005-2015 & Paediatric hospital & Yes \\
\hline Children's University Hospital - Temple Street & 2005-2015 & Paediatric hospital & Yes \\
\hline University Hospital Galway & 2005-2015 & & Yes \\
\hline University Hospital Limerick & 2005-2015 & 2013: returned $96.7 \%$ of discharges; 2014 : returned $97.2 \%$ of discharges & Yes \\
\hline University Hospital Waterford & 2005-2015 & & Yes \\
\hline Wexford General Hospital & 2005-2015 & & Yes \\
\hline
\end{tabular}


FIGURE A.1 INPATIENT AND DAY PATIENT BEDS PER 1,000 POPULATION AGED 65+, 2010-2015

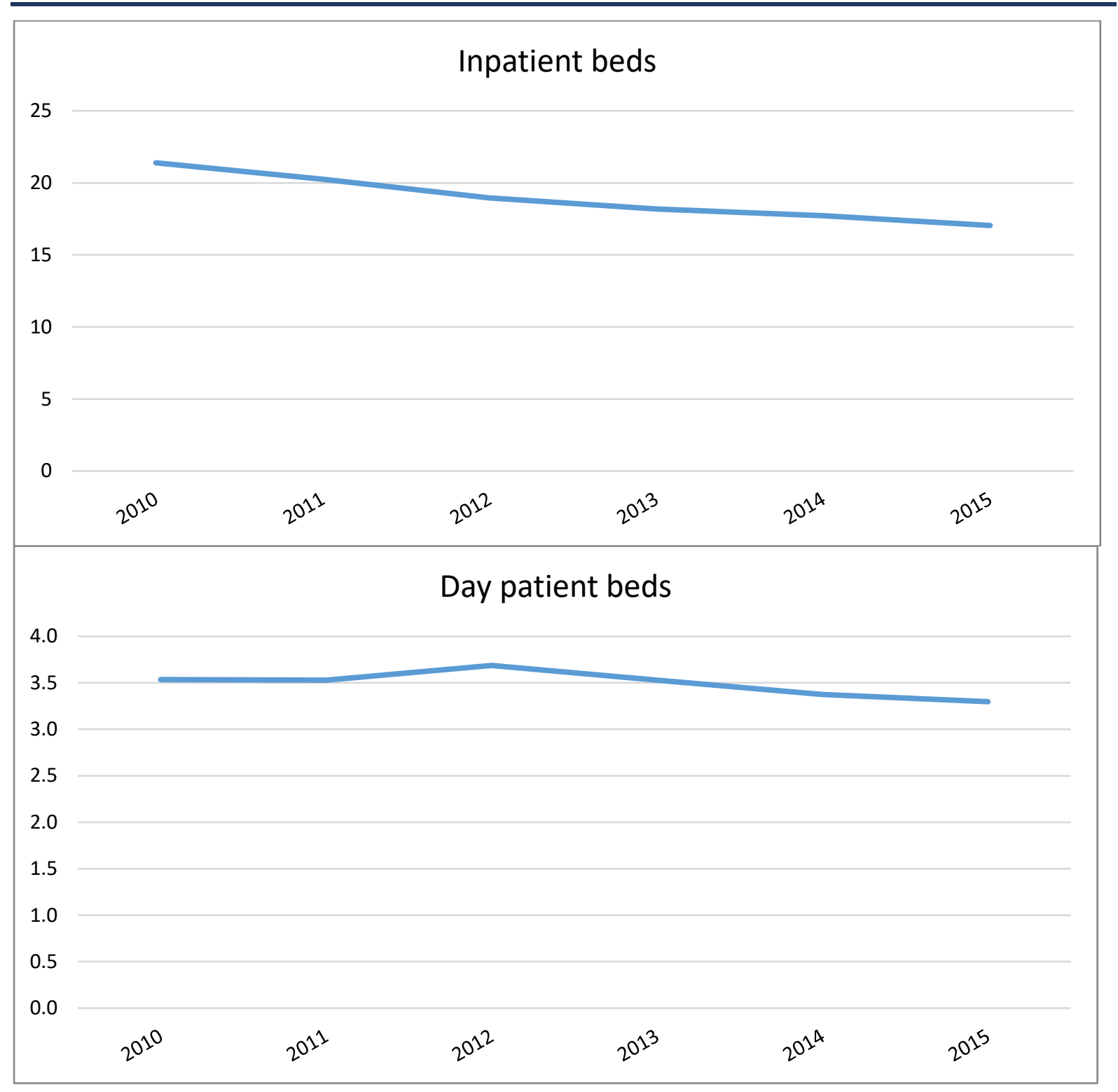


FIGURE A.2 PROPORTION OF DISCHARGES ACROSS MOST PREVALENT HOSPITALS OUTSIDE DUBLIN, 2015

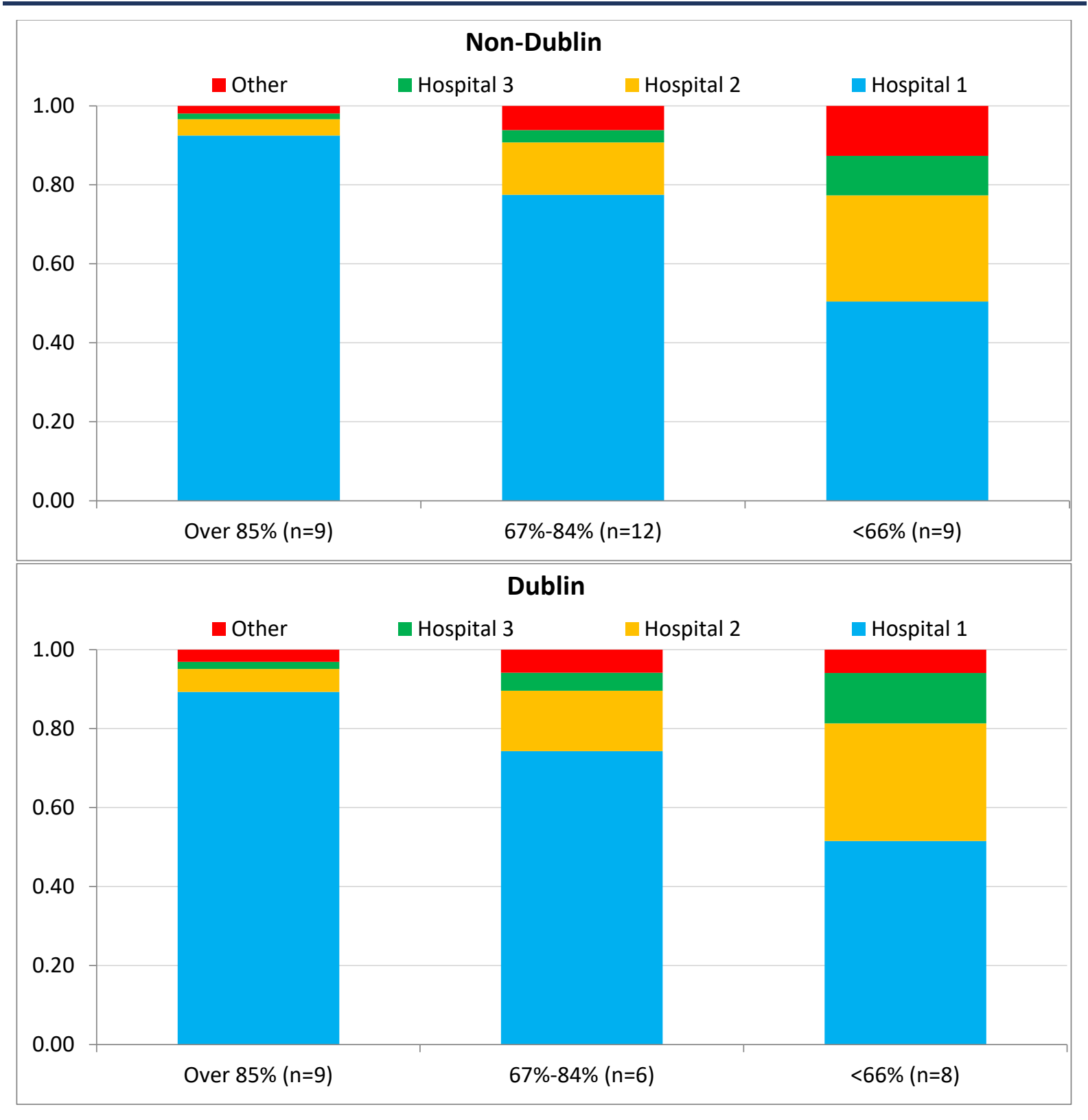




\section{A.1 CATCHMENT AREAS AND RECONFIGURATION}

In this sub-section, we examine how reconfiguration has affected catchment areas, and specifically how a closure or downgrade of a Tier 1 emergency department (ED) in the most dominant hospital for an area of residence results in neighbouring hospitals taking up the resultant demand.

To study the scenario outlined above, we examine areas where a dominant hospital has been reconfigured. Between 2005 and 2015, three areas of residence saw the hospital that provided the majority of emergency inpatient care reconfigured with the removal of its Tier 1 ED status. Figure A.3 illustrates the three areas affected; in 2005 the dominant hospitals provided 47 per cent, 52 per cent, and 56 per cent of emergency inpatient discharges respectively in each of the areas of residence. In 2015, following the reconfiguration of services, the previously dominant hospital saw the proportion of emergency inpatient discharges delivered considerably reduced. For each area, the previously second most dominant hospital (which, in each case, was geographically closest to the area of residence) became the dominant hospital. However, a number of other neighbouring hospitals also picked up many of each area's inpatient discharges.

FIGURE A.3 PROPORTION OF DISCHARGES ACROSS MOST PREVALENT HOSPITALS, 2005 AND 2015

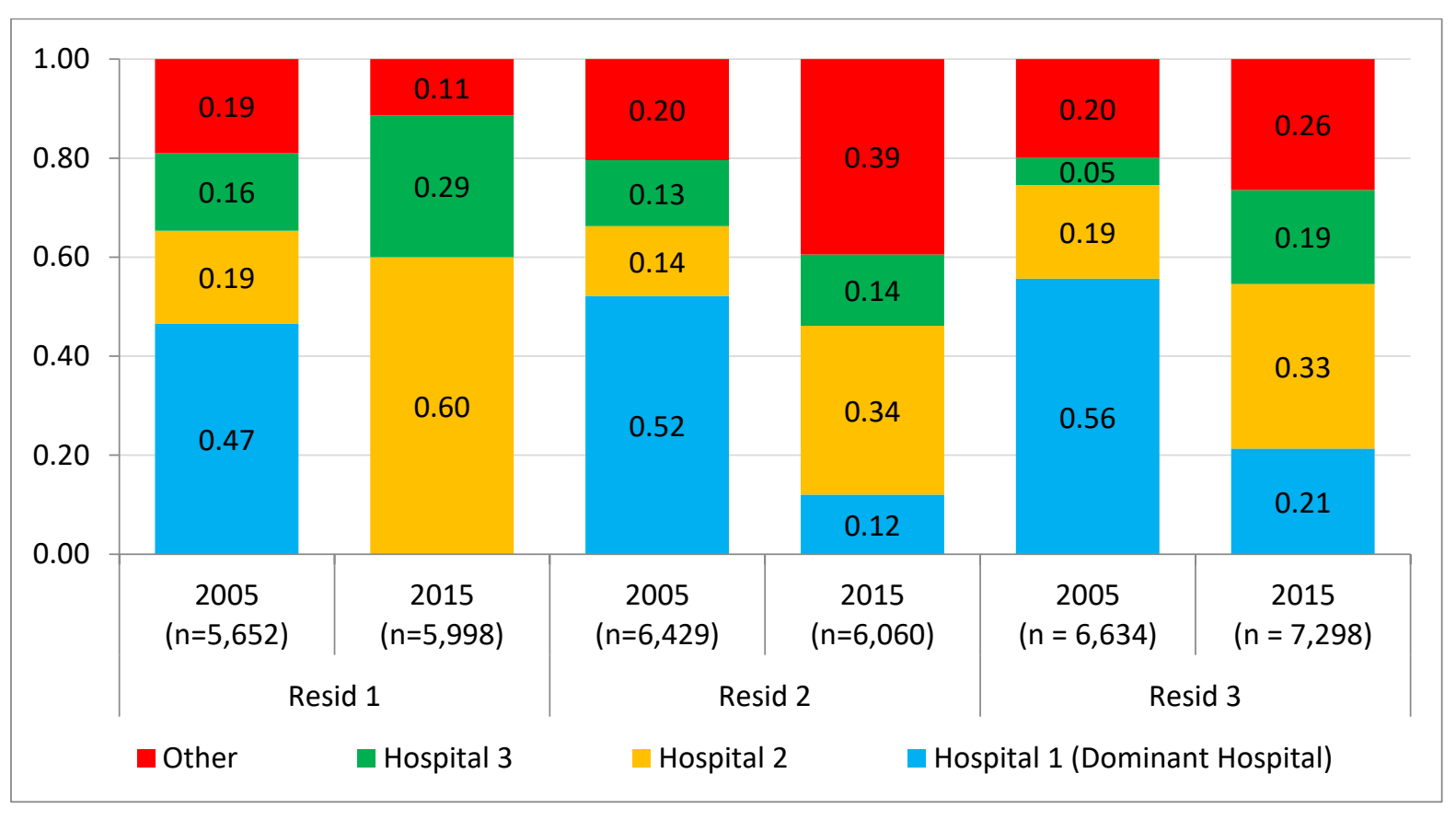




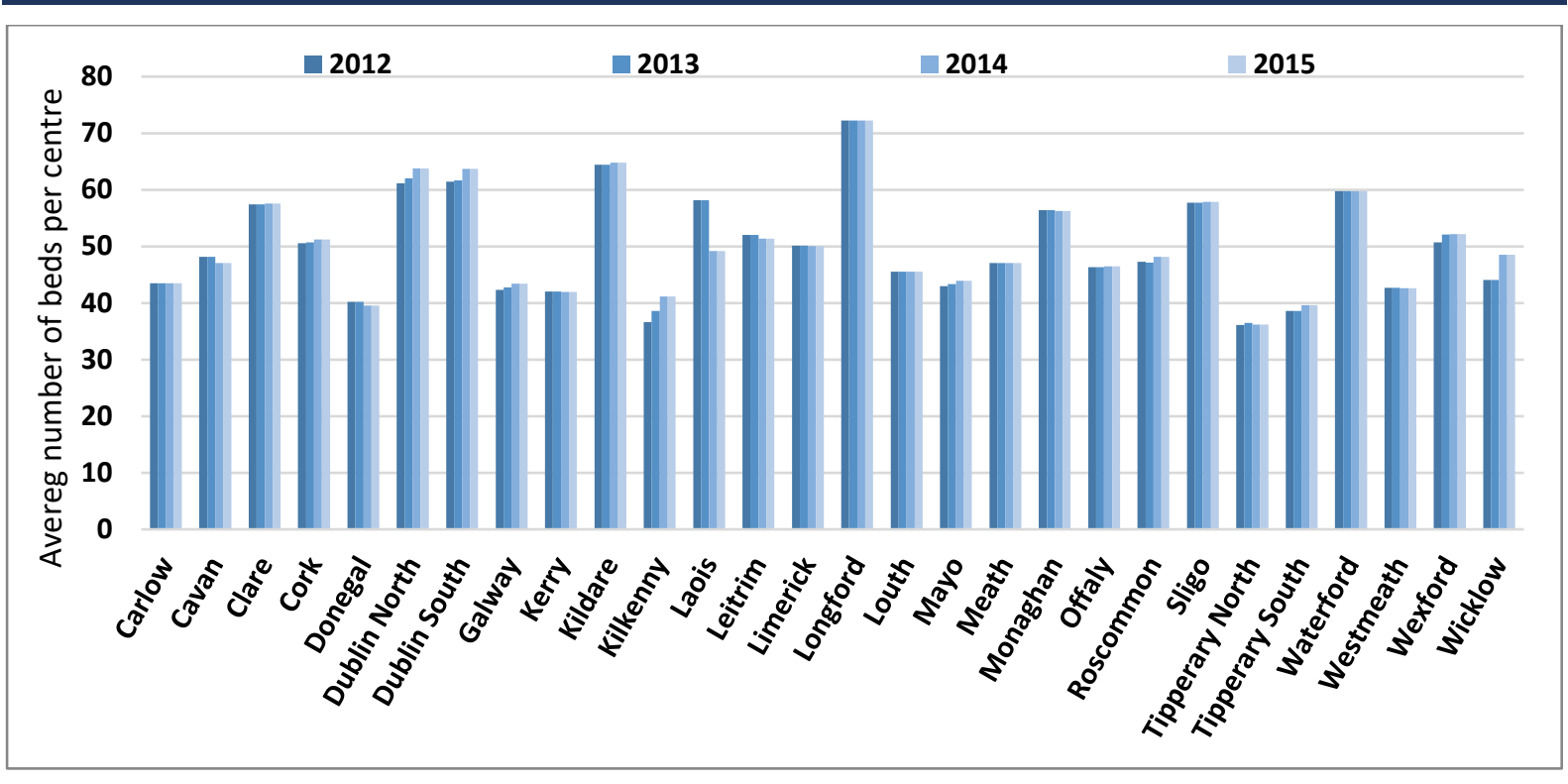

TABLE A.3 AVERAGE NUMBER OF BEDS PER LTRC CENTRE BY GEOGRAPHIC AREA, IRELAND 20122015

\begin{tabular}{|l|c|c|c|c|c|}
\hline Geographic area & $\mathbf{2 0 1 2}$ & $\mathbf{2 0 1 3}$ & $\mathbf{2 0 1 4}$ & $\mathbf{2 0 1 5}$ & \% change 2012-2015 \\
\hline Carlow & 44 & 44 & 44 & 44 & 0.0 \\
\hline Cavan & 48 & 48 & 47 & 47 & -2.3 \\
\hline Clare & 57 & 57 & 58 & 58 & 0.3 \\
\hline Cork & 51 & 51 & 51 & 51 & 1.2 \\
\hline Donegal & 40 & 40 & 40 & 40 & -1.6 \\
\hline Dublin North & 61 & 62 & 64 & 64 & 4.4 \\
\hline Dublin South & 61 & 62 & 64 & 64 & 3.7 \\
\hline Galway & 42 & 43 & 43 & 43 & 2.5 \\
\hline Kerry & 42 & 42 & 42 & 42 & -0.2 \\
\hline Kildare & 64 & 64 & 65 & 65 & 0.6 \\
\hline Kilkenny & 37 & 39 & 41 & 41 & 12.4 \\
\hline Laois & 58 & 58 & 49 & 49 & -15.5 \\
\hline Leitrim & 52 & 52 & 51 & 51 & -1.2 \\
\hline Limerick & 50 & 50 & 50 & 50 & -0.1 \\
\hline Longford & 72 & 72 & 72 & 72 & 0.0 \\
\hline Louth & 46 & 46 & 46 & 46 & 0.0 \\
\hline Mayo & 43 & 43 & 44 & 44 & 2.2 \\
\hline Meath & 47 & 47 & 47 & 47 & 0.0 \\
\hline Monaghan & 56 & 56 & 56 & 56 & -0.3 \\
\hline Offaly & 46 & 46 & 46 & 46 & 0.2 \\
\hline Roscommon & 47 & 47 & 48 & 48 & 1.8 \\
\hline Sligo & 58 & 58 & 58 & 58 & 0.2 \\
\hline Tipperary North & 36 & 36 & 36 & 36 & 0.2 \\
\hline Tipperary South & 39 & 39 & 40 & 40 & 2.8 \\
\hline Waterford & 60 & 60 & 60 & 60 & 0.0 \\
\hline Westmeath & 43 & 43 & 43 & 43 & -0.2 \\
\hline Wexford & 51 & 52 & 52 & 52 & 3.0 \\
\hline Wicklow & 44 & 44 & 49 & 49 & 10.1 \\
\hline Ireland & 50.5 & $\mathbf{5 0 . 6}$ & $\mathbf{5 0 . 9}$ & $\mathbf{5 1 . 2}$ & $\mathbf{1 . 4}$ \\
\hline Sord & & & & & \\
\hline
\end{tabular}

Source: HIQA (2012-2015), NHI, DoH LSAS. 
FIGURE A.5 COMPARISON OF MODEL FIT, POISSON AND NEGATIVE BINOMIAL

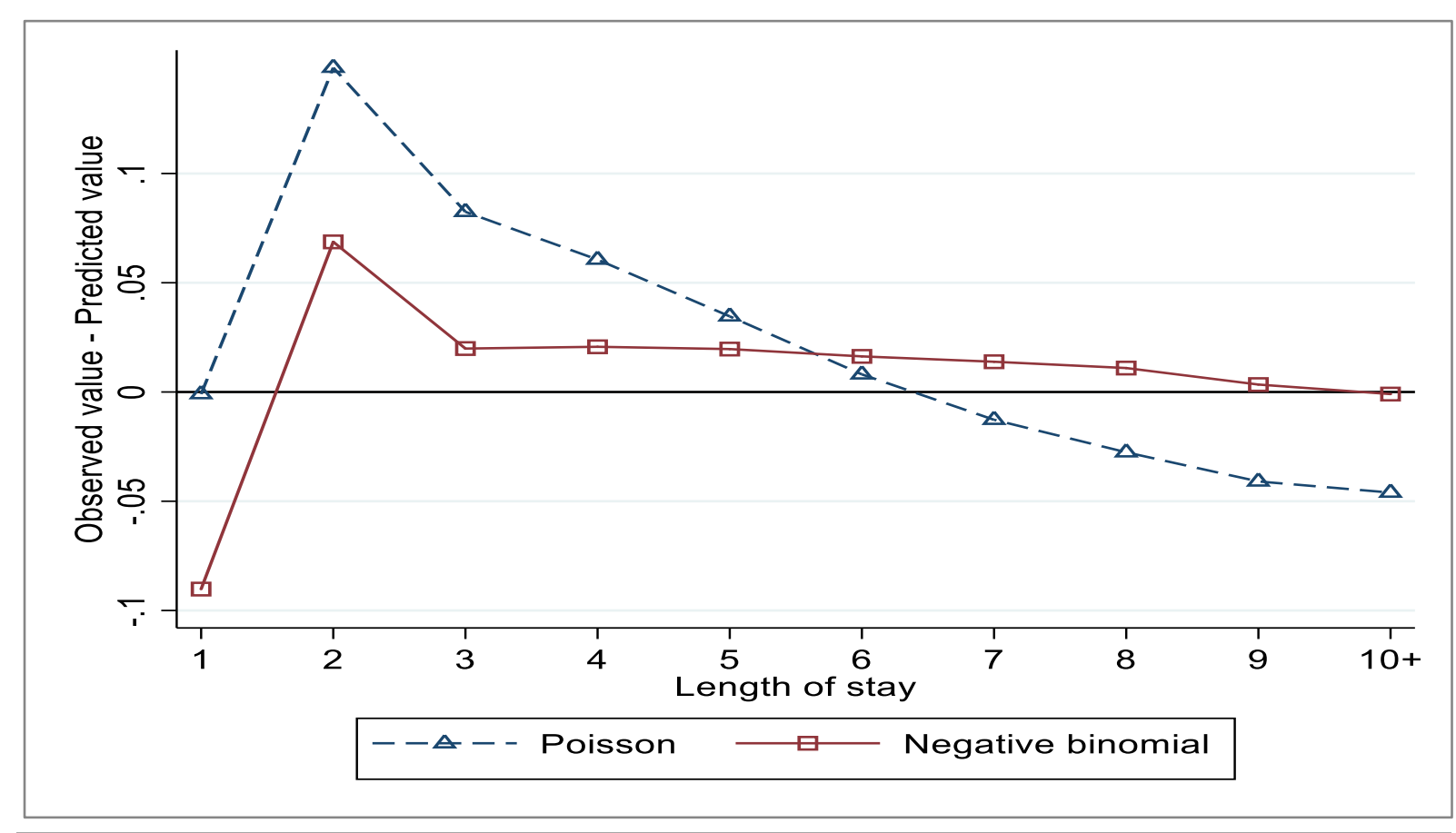


FIGURE A.6 UNCONDITIONAL QUANTILE REGRESSION FOR EMERGENCY INPATIENT DISCHARGES AGED 1844 YEARS, IRELAND AND DUBLIN NORTH, 2012-2015

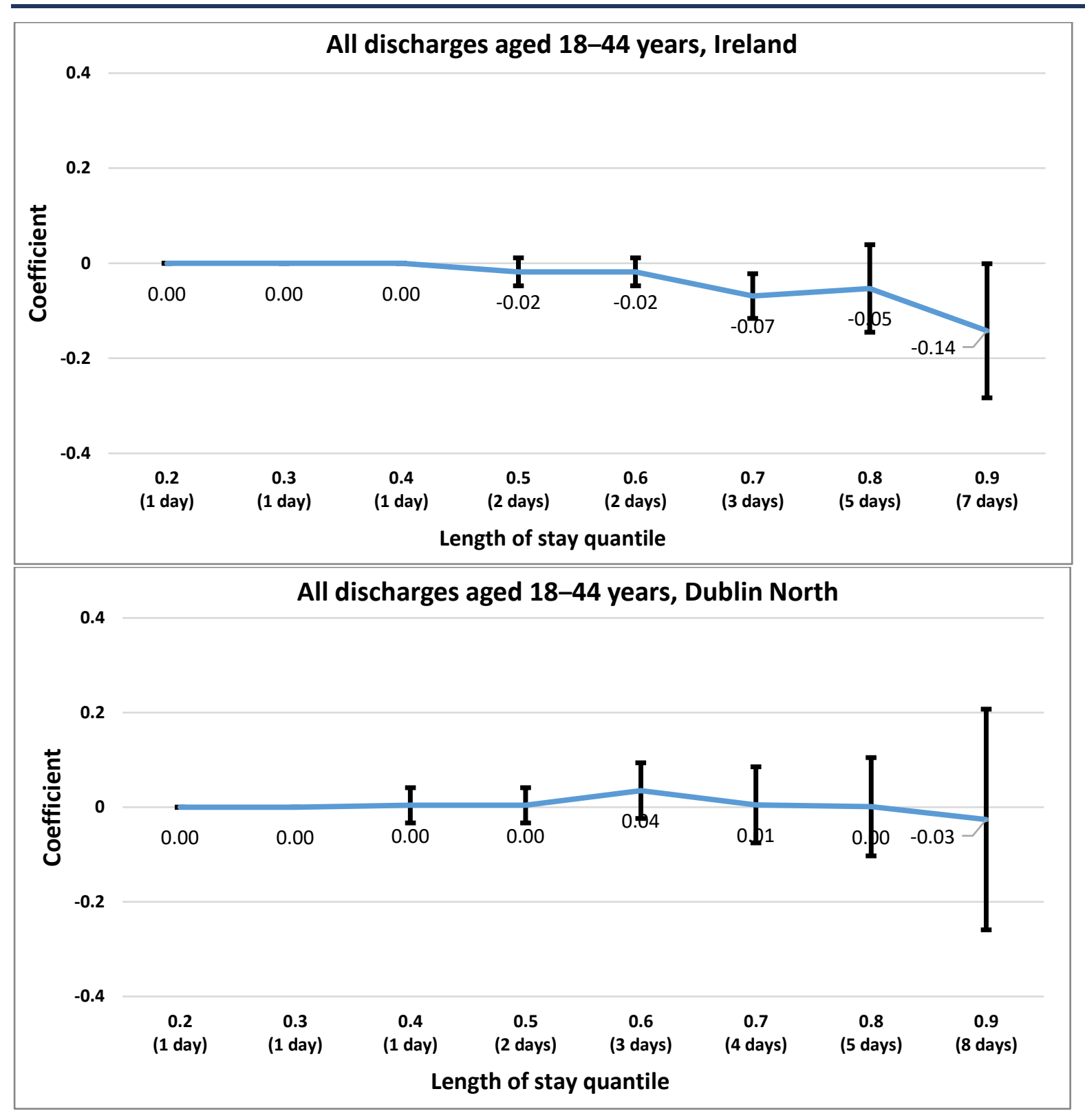



Whitaker Square,

Sir John Rogerson's Quay, Dublin 2

Telephone +35318632000

Email admin@esri.ie

Web www.esri.ie

Twitter @ESRIDublin

ISBN 978-0-7070-0500-3 